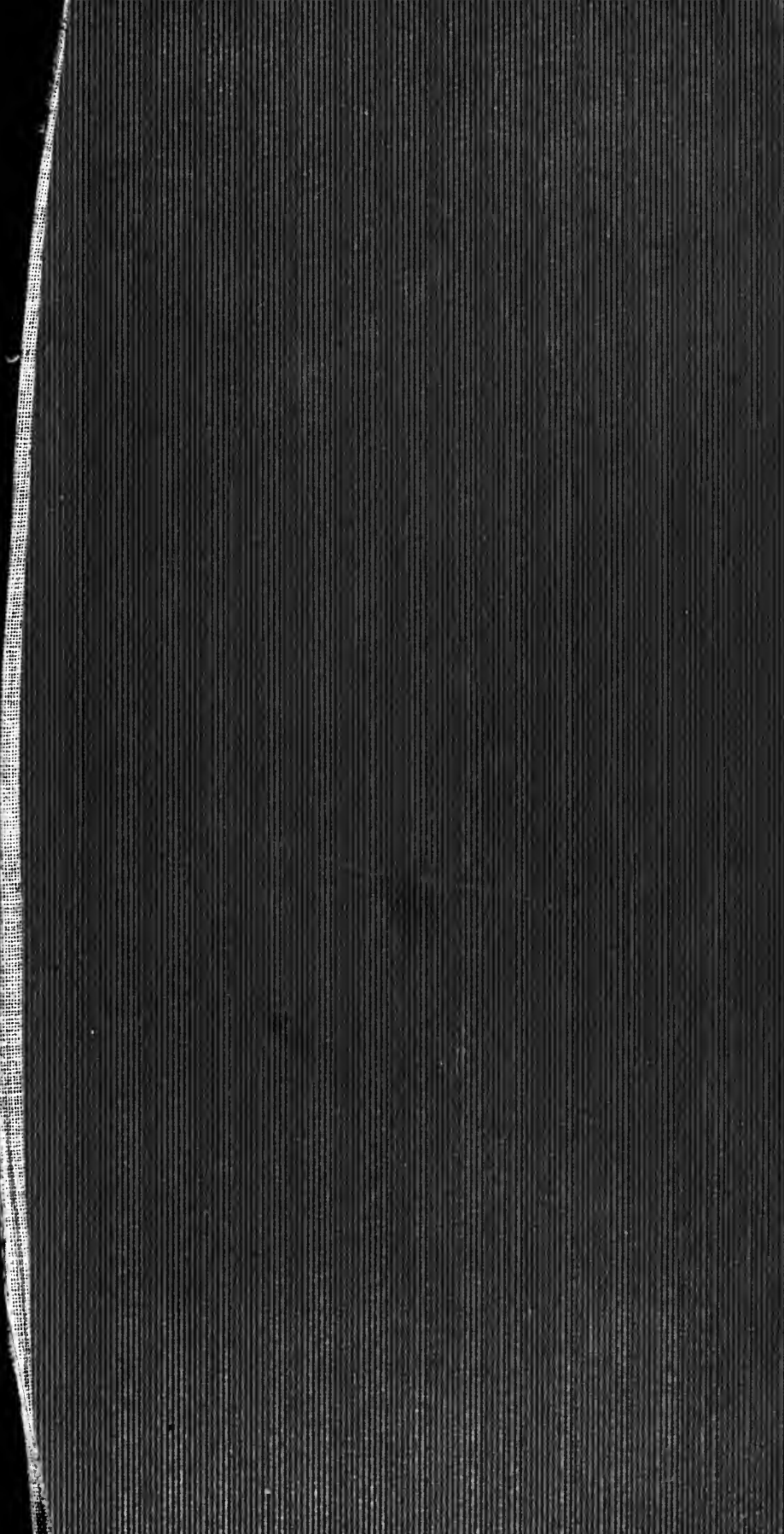


1283
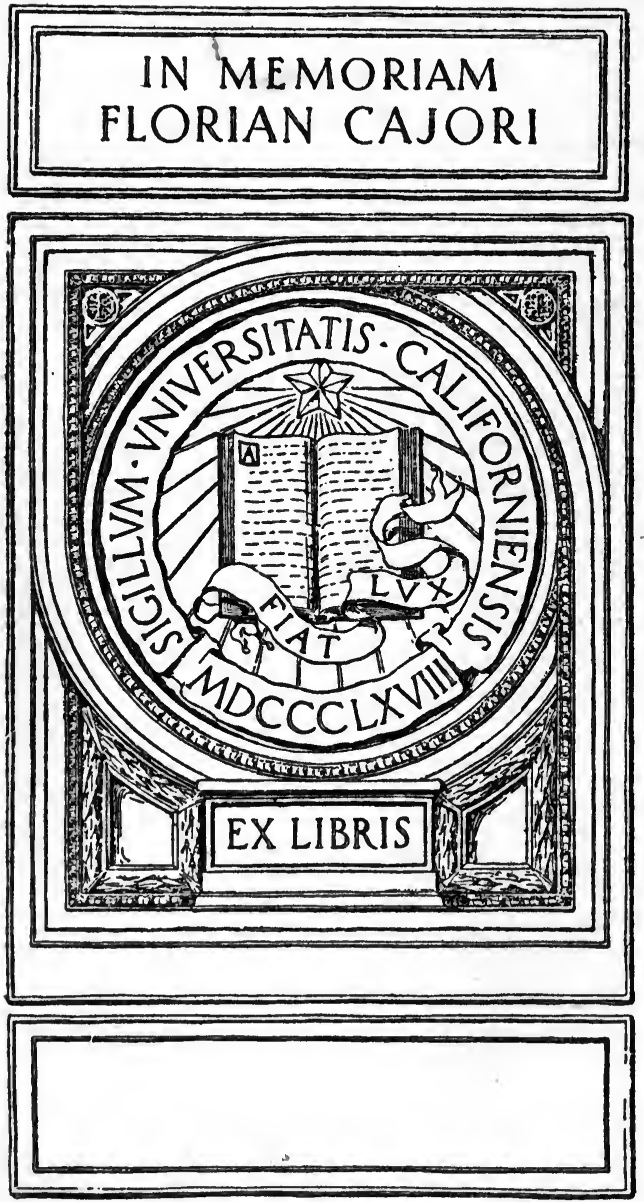
T. Cojori 
Digitized by the Internet Archive in 2007 with funding from Microsoft Corporation 


\title{
ELEMENTARY GEOMETRY
}

\author{
PLANE AND SOLID
}




$$
\text { The }>\text { X }
$$




\section{Q 4453 H 6}

Copyright, 1901,

BY THE MACMILLAN COMPANY.

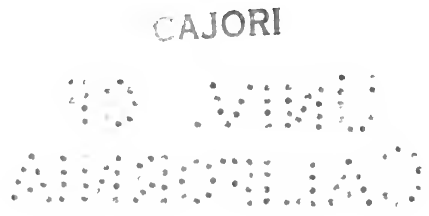

Norbood 租ress

J. S. Cushing \& Co. - Berwick \& Smith

Norwood Mass. U.S.A. 


\section{PREFACE}

Elementary Geometry deals only with forms whose determining parts are points, straight lines, and circles. Its method is that employed by the ancient writers. The amount of such study to be included in the high school or academy course has been fixed by tradition, as, in plane geometry, the equivalent of Euclid's first six books together with some additional material on mensuration; and, in solid geometry, the equivalent of Euclid's eleventh and twelfth books, to which is also added the mensuration of solids. Modern concepts and modern methods have given to this old material a correlation and a symmetry which it did not at one time present, and have opened fields of investigation which were entirely beyond the range of the early geometers. From the modern point of view, many isolated and apparently independent theorems have proved to be but special cases of broader and more general ones, or to be related to each other in an easily defined way. The so-called modern geometry possesses great beauty and strength, but how much of it can be wisely woven into a first course is a matter about which there is no general concensus of opinion. Some recent writers have deemed it wise to introduce general principles very early, while others have held rigorously to the old methods and old materials. My own belief is that the pupil must become quite familiar with the incidental and particular facts of geometry before he is capable of much generalization, and while I have written with the modern notions distinctly in mind, I have preferred not to depart far from the well-beaten path.

The attempt has been made to arrange the contents of this

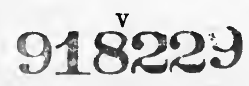


book in a natural and attractive pedagogical order. After the introductory chapter there are presented a few easy problems of construction in which the pupil will make free use of his ruler and compasses, and be introduced to the idea of a formal proof in connection with matters which he clearly sees need proving; other similar problems are inserted where they come in most naturally and are of most immediate interest.

Abstract discussions, such as the theory of limits, have been postponed as far as possible in order to secure for their comprehension greater mathematical maturity. Young pupils do not readily assimilate theoretical or abstract principles, and it is best that these should be brought into but little prominence in the early parts of the subject.

The exercises have been carefully selected and are intended to form an integral part of the work. They bear a direct relation, in most cases, to the propositions with which they are associated; those inserted at the ends of sections or chapters are for the most part of a more difficult character and may be omitted on a first reading.

It is hoped that the classified summary at the end of each chapter will prove serviceable for purposes of review and for reference, and will also aid the pupil in systematizing his knowledge.

Care has been taken to state the fundamental assumptions - the postulates - upon which the science rests, in as clear a form as possible, and to distinguish between assumption and axiomatic truth. Some things have been assumed which are often made matters of demonstration; for example, the fact that the perimeter of a regular polygon inscribed in a circle approaches a limit as the number of its sides is indefinitely increased. This admits of a rigorous proof, to be sure, but the proofs given in text-books on elementary geometry are as a rule either unsatisfactory or beyond the appreciation of the pupil. I have preferred openly to assume the property, - an assumption at which the pupil does not hesitate. 
A circle has been defined as a particular kind of line, and a polygon as a figure made up of points and lines. The area of such a figure is defined to be the surface enclosed by it. This accords, I believe, with the best usage, though perhaps not with the common usage. Everywhere outside of a class in elementary geometry a circle is so understood. Neither in more advanced mathematics nor in everyday life is it thought of as a portion of a plane, in accordance with the common definition, and I see no reason why the pupil should be obliged to change his idea of such a figure upon entering the geometry class, and change back again immediately upon leaving that class.

The Appendix contains a short chapter on Plane Trigonometry, which is intended to serve as a brief introduction to the subject, to meet the needs of those preparatory pupils who take up the study of physics or mechanics, not as a substitute for a complete course. It may be found sufficient also to meet the practical needs of those who do not continue their studies beyond the high school.

The teacher will do well to consider the following suggestions :-

1. Read the introductory chapter carefully, then talk it over with the class in an informal way, calling attention to the geometrical principles involved, most of which will be readily accepted by your pupils. Do not assign this chapter as a lesson.

2. Proceed very slowly at first. Remember that your pupils already have some geometrical ideas. Draw these out, clarify and fix them. Do not break down, but build on what your pupils already have.

3. Do not be too strenuous at first about a formal demonstration. Emphasize the geometric truth presented. Fix as your ideal an elegant, faultless proof, and gradually work up to it. 
4. Remember that in this subject the primary object should be the acquisition of geometric knowledge and the development of the geometric sense. I Logical reasoning and rhetorical demonstration are secondary aims, to be sure, but the first object should be Geometry.

Since my chief desire has been to produce a text-book adapted to the needs of the class-room, I have not hesitated to make free use of many existing texts, both old and new, and from them have derived much valuable help and many suggestions. The exercises in particular have been gathered from a variety of sources; only a few of them are new. To many friends, and in particular to my colleague, Professor Henry S. White, to Mr. B. Annis, of the Hartford, Connecticut, High School, and to Mr. J. F. Petrie, of North-western University Academy, I am greatly indebted for valuable suggestions and criticisms. Also to my pupil, Miss Elda L. Smith of Springfield, Illinois, my sincerest thanks are due for patient and careful work in testing all exercises and in the tedious task of proof-reading.

I shall be glad to be informed of any errors that may have been overlooked, and to receive suggestions for improvement either in matter or arrangement.

THOMAS F. HOLGATE.

Evanston, Illinois, May 30, 1901. 


\title{
CONTENTS
}

\author{
PART I \\ PLANE GEOMETRY \\ INTRODUCTION
}

Preliminary Notions and Definitions . . . . . . 1

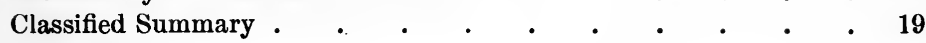

\section{CHAPTER I}

\section{Triangles and Parallelograms}

Section I. General Properties of Triangles . $\quad$. $\quad$. $\quad 22$

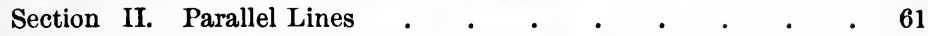

Section III. Closed Rectilinear Figures. Parallelograms . . 71

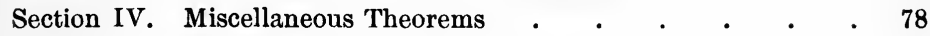

Section V. Additional Problems of Construction . $\quad$. $\quad . \quad$. 84

Section VI. Symmetrical Figures . . . . . . . . $\quad .88$

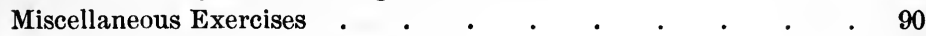

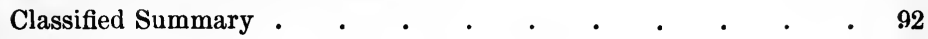

\section{CHAPTER II}

\section{The Circle}

Section I. Definitions and Preliminary Theorems _ . $\quad 98$

Section II. Angles inscribed in Arcs . . . . . . . 111

Section III. Secants and Tangents. Principle of Continuity . . 115

Section IV. Circles in Contact . . . . . . . 130

Miscellaneous Exercises . . . . . . . . . 134

Classified Summary . 


\section{CHAPTER III}

\section{Similar Rectilinear Figures}

Section 1. On Measurement, Ratio, and Proportion. Theory of Limits . . . . . . . . . 140

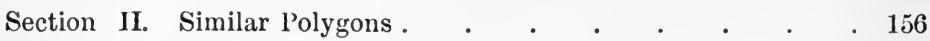

Section III. Problems of Construction . . . . . . . 174

Section IV. Additional Propositions. Harmonic Division . . 180

Miscellaneous Exercises . . . . . . . . . . . . 189

Classified Summary . . . . . . . . . . . . . 190

\section{CHAPTER IV}

Areas of Plane Polygons

Section I. Parallelograms and Triangles . . . . . . 194

Section II. Areas of Similar Polygons . . . . . . . 207

Section III. Problems of Construction . . . . . . . 220

Section IV. Maxima and Minima . . . . . . . . 226

Miscellaneous Exercises . . . . . . . . . . . 229

Classified Summary . $\quad . \quad$. $\quad$. . . . . . 230

\section{CHAPTER V}

Measurement of the Circle

Section I. Regular Polygons . . . . . . . 233

Section II. Measurement of the Circle . . . . . . 244

Section III. Problems of Construction . . . . . . . . 251

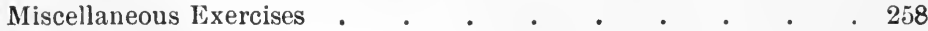

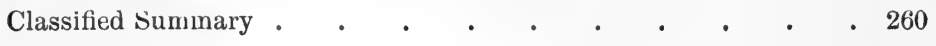

PART II

SOLID GEOMETRY

CHAPTER VI

Lines and Planes in Space

Section I. Intersecting Planes. Parallels and Perpendiculars . 263

Section II. IDihedral Angles . . . . . . . . 284

Section III. Polyhedral Angles . . . . . . . 290

Miscellaneous Exercises . . . . . . . . . 304

Classified Summary . $\quad . \quad$. $\quad . \quad$. $\quad$ • . . 305 


\section{CHAPTER VII \\ Prisms and Pyramids}

Section I. Area and Volume of a Prism .310

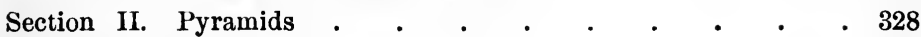

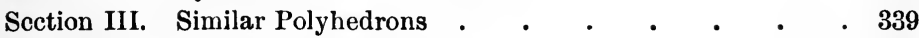

Section IV. Regular Polyhedrons . . . . . . . . . 342

Section V. Polyhedrons in General . . . . . . . $\quad$. 345

Miscellaneous Exercises . . . . . . . . . . 347

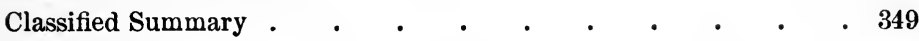

\section{CHAPTER VIII \\ Cylinders and Cones}

Section I. Cylinders . . . . . . . • • • 353

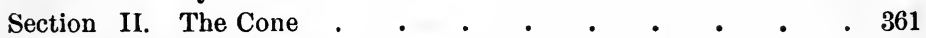

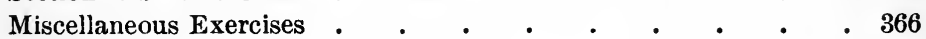

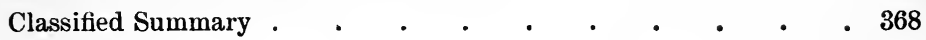

\section{CHAPTER IX}

\section{The Sphere}

Section I. Plane Sections and Tangent Planes . $\quad$ • $\quad$ - 370

Section II. Spherical Angles, Triangles, and Polygons . . $\quad$. 380

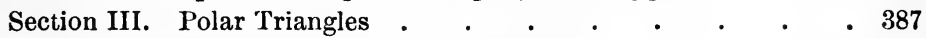

Section IV. Areas of Spherical Triangles . $\quad . \quad$ • $\quad . \quad .394$

Section V. Volume of the Sphere . . . . . . . 405

Miscellaneous Exercises . . . . . . . . . . . . 409

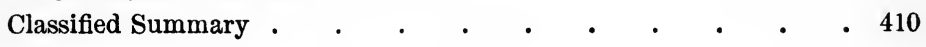

\section{APPENDIX}

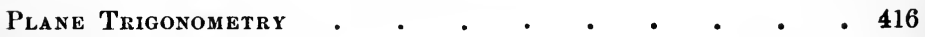

\section{TABLES}

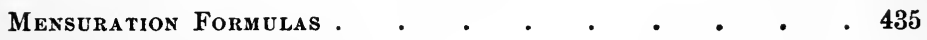

INDEX to Definitions . . . . . . • • • . . 437 



\section{PART I-PLANE GEOMETRY}

\section{$\longrightarrow \infty+\infty 0200$ \\ INTRODUCTION}

\section{PRELIMINARY NOTIONS AND DEFINITIONS}

When we speak of a point or a line, a straight line or a crooked line, everybody knows in a general way. what is meant. In the minds of persons not familiar with the strict sense in which these terms are used in Geometry, it may be that the words 'point' and 'line' are associated with marks of some sort, made for example with a pen or pencil; they may think of a point as a dot, and of a line as a long, narrow mark, but in spite of this association the true meaning of the terms ' point,' ' line,' 'straight,' 'crooked,' etc., is pretty clearly understood.

It is not at all certain that definitions could be given in words which would convey an intelligent idea of the meaning of these terms to a person not already familiar with the thought conveyed by them, still a few words of comment may serve to fix the attention upon some properties of points and lines which are fundamental in the study of Geometry.

1. A point has no magnitude; it has only position.

The smallest mark that can be made with the finest pencil or pen will have some magnitude, and consequently is not a true point in the sense in which this term is used in Geometry. We can think. of points as having position only, but in making diagrams we are obliged to use pencil or pen marks to represent them. 
[INTROD.

2. A line has position and length, but no breadth or thickness.

Whenever we speak of a line it suggests the idea of extension in one way, namely, length, without regard to breadth or thickness; but if we try to represent a line by a mark, this mark will of necessity have some breadth and so cannot be a true geometrical line. You should endeavor to think of a line without actually drawing a mark to represent it.

A line is sometimes spoken of as the path of a moving point.

On a line you can choose as many points as you please. Through two points you can draw as many lines, of one kind or another, as you please.

3. In order to tell one point from another we give them names, conveniently the names of the letters of the alphabet, and place the letter by the side of the point bearing its name.

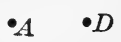

Thus we speak of the point $A$, the point $B$, etc.

If we wish to designate different points by the - $C$ same letter, $P$ say, we distinguish them as $P_{1}, P_{2} ; P_{3}$, and so on.

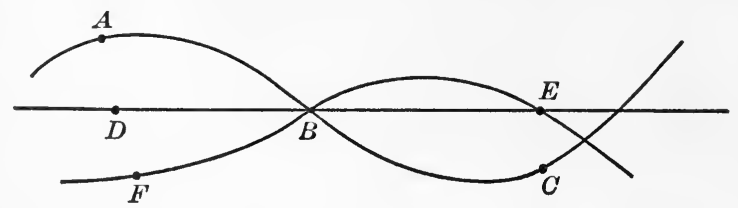

We denote a line by naming a sufficient number of points on it to distinguish it from every other line.

Thus we have in the diagram the line $A B C$, the line $D E$, the line $F B E$.

The two points $B$ and $E$ would not distinguish the line $D B E$ from the line $F B E$, since $B$ and $E$ are points of both lines, or as we say, are common to both

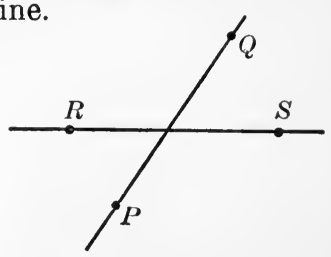
lines. The lines $P Q$ and $R S$ in the second diagram are, however, distinguished by naming two points on each. 
4. A straight line is such that only one of that kind can pass through the same two points.

That is to say, if two straight lines pass through the same two points, they must occupy the same position, and there is no point of one which does not also lie on the other.

This is sometimes expressed by saying that, "If two straight lines coincide at two points, they coincide throughout"; or, "Two points determine a straight line," i.e. fix the location of the line and distinguish it from every other straight line.

Two different straight lines therefore can have only one point in common; in other words, two straight lines intersect in only one point.

Any two straight lines can be made to coincide at every point by placing them so that they coincide at two points.

5. Suppose that on a given straight line we choose two points and mark them by the letters $A$ and $B$. These will serve to distinguish this straight line

from every other, since no A

$B$ other straight line can pass

through both of these points. We may therefore call this line the straight line $\boldsymbol{A B}$.

That portion of the line which lies between the points $A$ and $B$ is called the segment $\boldsymbol{A} B$ of the line. If other points are chosen on the line,

as $C, D, E$, etc., the portion of the line lying between the points $A$ and $C$ is called the segment $A C$; that between $C$ and $D$, the segment $C D$, etc.

It should be observed that two points chosen anywhere on a straight line determine it; the line may be as long as you please, i.e. it may be unlimited in extent, yet these two points will distinguish it from every other straight line. When we have in mind only that segment of a straight line lying between 
[INTROD.

two fixed points $A$ and $B$, we shall usually speak of it as the line-segment $\boldsymbol{A B}$.

The distinction thus made between the straight line and the line-segment is sometimes effected by calling the former an unlimited straight line, and the latter a terminated straight line. Of course any mark that we make to represent a straight line must be terminated both ways by points; but the term 'line-segment' will be used only when we wish to restrict our thought to that portion of the whole line which lies between two particular points.

6. When two line-segments having one end-point in common lie in the same straight line and do not overlap, for example, the segments $A C$ and $C B$, then the segment which is made up of these two, i.e. the segment $A B$, is called their sum.

If the line-segments have one end-point in common and do overlap, then that segment of the one which is not covered by the other is called their difference.

Thus the difference of the segments $A B$ and $C B$, in the last diagram, is the segment $A C$.

7. A surface extends in two ways, i.e. it has length and breadth, but no thickness.

We speak of the surface of the blackboard, the surface of the table, the surface of the earth, and think of their extension in two ways only, not at all of depth or thickness. A geometrical surface has no thickness.

Any mumber of lines can be drawn on a surface, and any number of points can be chosen on a surface.

If a surface is such that every straight line joining two points of it lies wholly on the surface, it is called a plane surface. 
The top of a table is approximately a plane surface, while the surface of an apple is clearly not plane.

For brevity we sometimes use the term a plane instead of a plane surface.

8. A solid extends in three ways, i.e. it has length, breadth, and thickness.

9. A geometrical figure is any combination of points, lines, and surfaces.

A plane geometrical figure is one whose points and lines all lie in one plane.

\section{Triangles and Angles}

10. One of the simplest geometrical figures is what is called a triangle. It consists of three straight lines which intersect two and two in three points.

The three points are called the vertices of the triangle, and the three straight lines its sides.
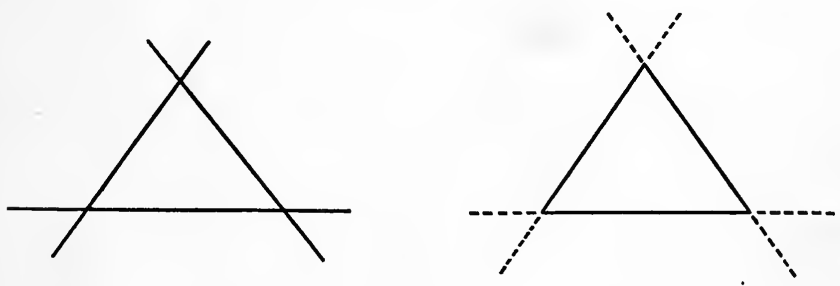

In most cases when we speak of a triangle we shall have in mind only the figure consisting of the three vertices and the three line-segments lying between them, and then the three line-segments are thought of as the sides of the triangle. It should be remembered, however, that these sides may be extended as far as we wish at any time. 
[INTROD.

The length of a side will always mean the length of the linesegment between the vertices.

A triangle may be designated by naming its vertices since no different triangle can have the same three vertices.

Thus the triangle in the diagram is the triangle $A B C$, or the triangle $B A C$.

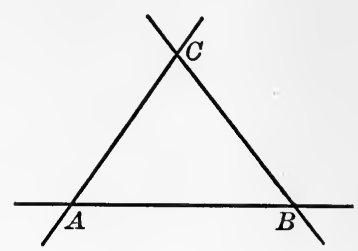

Exercise. Name the triangle in the diagram in six different ways.

Sometimes it is found convenient to designate a straight line by a single letter, and in this case we commonly use a small letter, reserving the capital letters to denote points. When the sides of a triangle are so marked we can designate the triangle by naming its three sides.

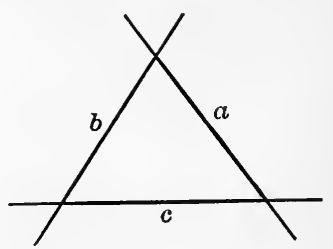

11. In looking at the figure of a triangle it will be noticed that the two sides which meet in any vertex start out from the vertex in different directions.

Whenever two straight lines which meet diverge from their common point in this way, they are said to form a plane angle, or simply an angle, with each other.

The idea of an angle will be made clearer by the accompanying figure. The straight lines $A B$ and $A C$ meet at the point $A$. They make with each other an angle at this point. This angle we name the angle $B A C$, or the angle $C A B$, meaning by that the angle formed by the lines $B A$ and $A C$. It is

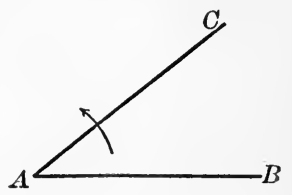
sometimes spoken of as "the angle between the lines $A B$ and $A C$," or " the angle contained by the lines $A B$ and $A C . "$

If we keep the line $A B$ fixed in position and rotate the line 
$A C$ about the point $A$ in the way indicated by the arrowhead, we enlarge the angle $B A C$; that is, we increase the divergence between the lines $A B$ and $A C$, and so enlarge the angle. If, however, we rotate the line the other way, the divergence becomes less; that is, the angle formed by the lines becomes smaller.

The size of the angle formed by two lines does not depend on the length of the lines, or on anything except the amount of their divergence.

The straight lines $A B$ and $A C$ drawn from $A$ are called the boundaries of the angle, and their point of intersection $A$, the vertex of the angle.

It will readily be seen that there are three angles in any triangle, namely, the angle made by the sides $A B$ and $A C$ at $A$, that made by the sides $B A$ and $B C$ at $B$, and that made by the sides $C A$ and $C B$ at $C$. For brevity we shall sometimes speak of these as the angle $A$, or the angle $B$, or the angle $C$, but it must always be borne in mind that we mean the angle at $A$, or $B$, or $C$, made by the two straight lines which meet at that point.

The pupil should carefully observe that in reading an angle the letter at the vertex is always named between the other two letters. The angle $B A C$ and the angle $C A B$ are the same, having the same vertex at $A$ and the same boundaries $A B$ and $A C$, while the angle $A B C$ is different, having its vertex at $B$.

12. When two angles are placed so as to have the same point for vertex and one boundary in common without overlapping, the angle formed by the other two boundaries, of which these two angles are parts, is called the sum of the two angles.

If, on the other hand, the two angles have the vertex and one boundary in common and do overlap, then the angle formed by the other two boundaries is called the difference of the two given angles. 
For instance, the angle $B A D$ in the figure is the sum of the angle $B A C$ and the angle $C A D$, while the angle $B A C$ is the difference of the angle $B A D$ and the angle $C A D$.

When two angles have a common vertex and one common boundary, without overlapping, they are called adjacent angles.

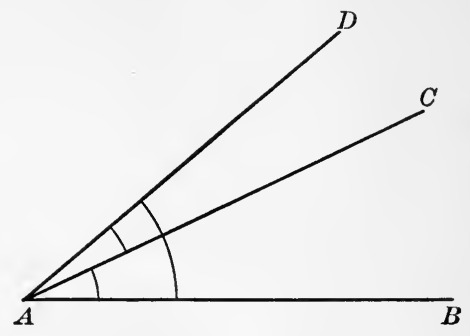

Thus in the figure the angle $B A C$ and the angle $C A D$ are adjacent angles.

13. Suppose that on a straight line $A B$ we choose any point $C$, and from it draw another straight line $C D$ (Fig. 1). This line makes two adjacent angles with $A B$, namely, the angle $B C D$ and the angle $D C A$. If the line be drawn, as $E D$ (Fig. 2), so as to cross $A B$, then it also makes with $A B$ two adjacent angles on the other side, namely, the angle $E C B$ and the angle ECA.

In Fig. 2 the angles whose boundaries lie in the same straight line, but which have only their vertices in common, for example, the angle $A C E$ and the angle $B C D$, are called vertically opposite angles, or briefly, vertical angles.

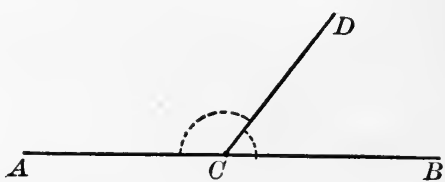

FIG. 1

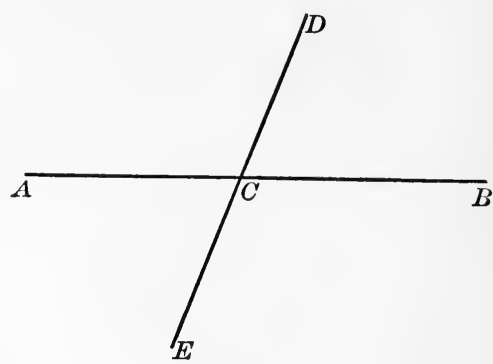

FIG. 2

Exercise. In this figure point out and name all pairs of adjacent angles and all pairs of vertically opposite angles. How many pairs of each kind do you find? 


\section{Test of Equality}

14. In Elementary Geometry we assume that figures can be moved about in any manner without altering their size or shape. Upon this assumption we have the following tests of equality :

1. If two line-segments can be so. placed that their endpoints coincide, they are equal; and conversely, if two linesegments are equal, they can be placed so that their end-points will coincide.

2. If two plane angles can be so placed that their boundaries coincide, they are equal ; and conversely, if two plane angles are equal, they can be placed so that their boundaries coincide.

3 . If two triangles can be so placed that their vertices coincide, and consequently also their sides and angles, they are identically equal; and conversely, if two triangles are identically equal, they can be placed so that their vertices, their sides, and their angles will coincide.

In general, two geometrical figures are said to be identically equal when, and only when, they can be made to occupy, point for point, and line for line, the same positions.

\section{Right Angles and Straight Avgles}

15. Suppose that from any point $C$ in a straight line $A B$, a straight line $C D$ is drawn so as to make the adjacent angles $B C D$ and $A C D$ equal, then each of these equal angles is called a right angle, and $C D$ is said to be at right angles to $A B$, or perpendicular to $A B$.

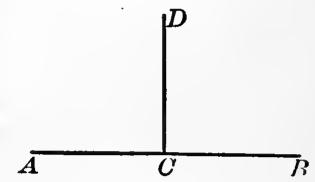

Definition. If one straight line meets another so as to make the two adjacent angles equal, each of these angles is called a right angle. 
16. Let us take any straight line $A B$ and another straight line $C D$ meeting it at the point $C$ so as to make two adjacent angles $A C D$ and $B C D$. If now we let the line $A B$ remain fixed in position, and rotate the line $C D$ about the point $C$ so that it takes up successively the positions $C D_{1}, C D_{2}, C D_{3}^{\circ}$, etc., the

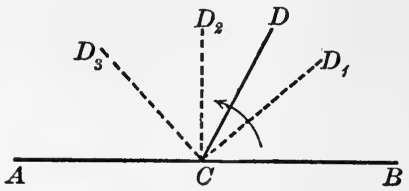
effect will be to gradually increase one of the two adjacent angles made by the lines, and to decrease the other.

The sum of the two angles however will remain unaltered, since the boundaries of the sum, viz. $C B$ and $C A$, are not changed by the rotation of $C D$.

In some one position, as at $C D_{2}$, each of the adjacent angles is a right angle, and their sum is, therefore, two right angles. Hence in whatever position the line $C D$ is drawn the sum of the two adjacent angles $B C D$ and $A C D$ is always equal to two right angles.

If the line $C D$ should cross $A B$, the sum of the two angles on the other side of $A B$ would also be equal to two right angles.

That is to say,

The sum of the two angles which one straight line makes with another on one side of it is equal to two right angles; and when two straight lines intersect, the sum of the four angles formed at their point of intersection is equal to four right angles.

17. The pupil should notice carefully the argument which led up to the above conclusion.

(1) The straight line $C D$ makes two adjacent angles with the straight. line $A B$, which vary in size as $C D$ rotates about $C$.

(2) The sum of these adjacent angles does not vary by the rotation of $C D$.

(3) For one position of $C D$, each of the adjacent angles is a right angle, and consequently their sum is equal to two right angles.

(4) Therefore for every position of $C D$, the sum of the two adjacent angles is equal to two right angles. 
Finally, the argument which applies to the two angles on one side of $A B$ would apply equally well to the two angles on the other side, if the line $C D$ were drawn so as to cross $A B$ at $C$.

18. From Article 16 it follows at once that - If from a point in a straight line any number of straight lines are drawn in a plane on one side of the line, the sum of all the angles so formed is equal to two right angles; and,

If any number of straight lines are drawn from a point in a plane, the sum of all the angles so formed is equal to four right angles.

19. When the sum of two angles is equal to two right angles, each of them is called the supplement of the other.

If two supplementary angles are not equal, one of them must be less than a right angle, and the other, greater than a right angle.

An angle which is less than a right angle is called an acute angle.

An angle which is greater than a right angle and less than two right angles is called an obtuse angle.

An acute angle is always less than its sup-

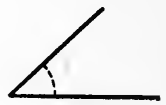
plement, while an obtuse angle is greater than its supplement.

The supplement of an acute angle is obtuse, and the supplement of an obtuse angle is acute.

What is the supplement of a right angle?

20. Suppose again we let one boundary $O B$ of an angle $B O C$ remain fixed while we rotate the other boundary $O C$ about the vertex so as to increase the angle. In one position, say the position $O C_{1}$, the two boundaries are in the same straight line.

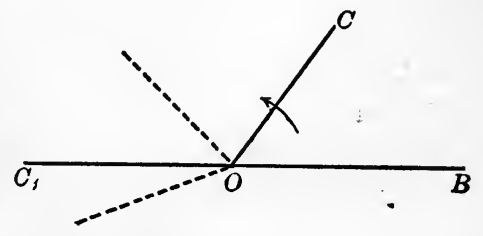


The angle $B O C$ has then become a so-called straight angle, and if $O C$ continues to rotate beyond this position the angle becomes reflex.

Definitions. A straight angle is an angle whose boundaries extend from the vertex in opposite ways, and lie in the same straight line.

A reflex angle is an angle which is greater than a straight angle.

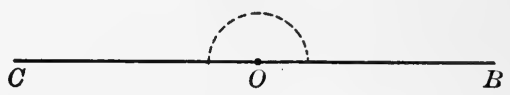

A straight angle is equal to the sum of two right angles; in other words, a right angle is half of a straight angle.

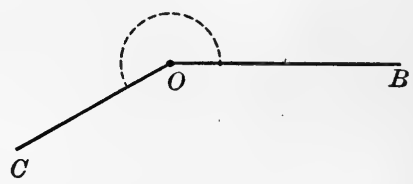

21. If we place one straight angle upon another so that their vertices coincide, and so that one boundary of the first shall coincide with one boundary of the second, then the other boundary of the first must also coincide with the other boundary of the second. For the two boundaries of each angle lie in a straight line, and these straight lines have been made to coincide in more than one point. Hence they must coincide throughout. Art. 4.

Therefore any one straight angle is equal to any other straight angle; or, all straight angles are equal.

Since a right angle is always half of a straight angle, and all straight angles are equal, therefore all right angles are equal.

The sum of any angle and its supplement is a straight angle. Hence, if two angles are equal their supplements are equal, and two different supplements of the same angle are equal.

22. Let us now take two supplementary angles $A O C$ and $B O C$ and place them so that they will be adjacent, that is, so that their vertices will coincide and one boundary of one will fall on one boundary of the other, while the angles do not overlap. 
The other boundaries $A O$ and $O B$ must then be parts of the same straight line.

For, if we should prolong the straight line $A O$ through the vertex, the angle so formed with $C O$ would be the supplement of $A O C$. But $B O C^{\prime}$ is the supplement of

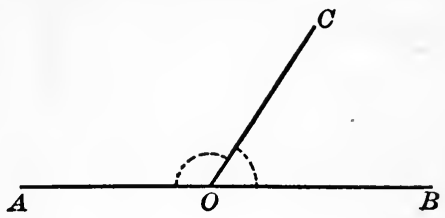
$A O C$. Therefore $O B$ must coincide with the extension of $A O$ through the vertex. That is to say,

If two adjacent angles are supplementary, their outer boundaries lie in the same straight line.

\section{On Closed Lines}

23. By a closed line we mean one that can be followed with a pencil or a pen, starting from any point of it and returning to the same point, without removing the pencil or pen from the paper, and without passing twice over the same portion of the line.

Any figure which can be traced in a similar

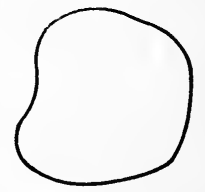
way, starting at one point and returning to the same point, is called a closed figure.
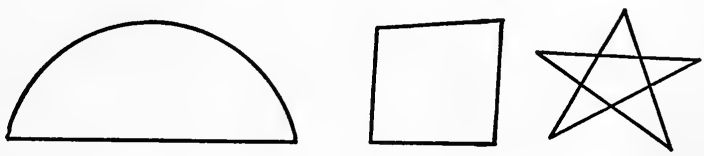

If a closed figure is made up wholly of segments of straight lines, it is called a closed rectilinear figure.

Any connected set of linesegments which is not closed may be called a broken line.

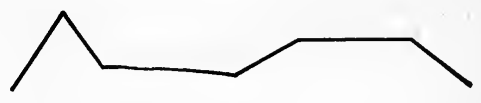




\section{Properties of Closed Lines}

24. If a point is chosen inside of any closed line, every straight line drawn through it must, if extended far enough, cut the closed line in at least two points.

If one closed line lies partly inside and partly outside of another closed line, these two closed lines must intersect in at least two points.

25. Definition. A circle is a closed line so drawn that all points of it are equally distant from a certain fixed point within it.

This fixed point is called the centre of the circle.

A line-segment drawn from the centre to any point of the circle is called a radius of the circle, while a line-segment drawn through the centre and terminated both ways by the circle is called a diameter of the circle.

A diameter of a circle is twice as long as a radius, or is equal to the sum of two radii.

If a straight line intersects a circle, it must cut it at two points if extended far enough, and when one circle lies partly within and partly without another, they must intersect at two points.

\section{ON THE USE OF INSTRUMents}

26. In the construction of figures in Elementary Geometry it is customary to limit ourselves to the use of a straight-edge or ruler and a pair of compasses.

With the ruler we are able to draw a straight line between any two points and also to extend or produce, as far as we please, any terminated or finite straight line or line-segment already drawn.

The ruler is supposed to have no scale of measurements marked on it, so that it cannot be used to compare lengths. 
The compasses are used for describing circles and for marking off equal lengths.

The pupil will readily see how this instrument can be made use of -

(1) To describe a circle having a given point for centre and its radius equal to a given line-segment.

(2) To mark off on the longer of two given line-segments a part equal to the shorter.

(3) To draw from a given point a line-segment equal to a given line-segment.

\section{Postulates}

27. In geometry, whenever we assume a fundamental property or the possibility of constructing some simple figure, we say that we postulate these things.

A few of these fundamental assumptions or postulates have already crept into our discussions along with the definitions and descriptions of simple figures.

\section{Examples of Postulates}

(1) A straight line can be drawn from any one point to any other point.

From the defining property of a straight line there is only one such line. Art. 4.

(2) A terminated straight line, or a line-segment, can be produced to any length in the same straight line.

(3) A circle can be described having any given point for centre and its radius of any given length.

(4) Geometrical figures can be moved about in space without altering their size or shape.

Other postulates will be added as we proceed, and these will be collected in tabulated form at the end of the chapter in which they first appear. 


\section{Axions}

28. A simple statement or proposition which is readily admitted to be true as soon as its meaning is understood, is called an axiom. In other words, an axiom is a statement the truth of which does not need to be demonstrated, and, in general, cannot be demonstrated; it is, as we say, self-evident.

Many of the axioms to which we shall make reference apply not only to geometrical magnitudes, but to magnitudes of all kinds.

The term 'magnitude' is applied to anything that has size, i.e. to anything that can be increased or diminished. For example, a line-segment, an angle, the surface within a closed figure, are magnitudes. A point is not a magnitude, since it has no size.

The following are a few examples of such axioms, but it must not be supposed that this list includes all that could be stated. Other axioms will be announced when there is occasion for their use.

\section{Examples of Axioms}

1. Magnitudes which are equal to the same magnitude are equal to each other.

If $A$ equals $C$, and $B$ equals $C$, then $A$ equals $B$.

2. If equal magnitudes are added to equal magnitudes the sums are equal.

If $A$ equals $B$, and $C$ equals $D$, then the sum of $A$ and $C$ equals the sum of $B$ and $D$.

3. If equal mugnitudes be taken from equals, the remainders are equal.

4. If equal magnitudes be added to unequals, or unequals to equals, the sums are unequal.

5. If equal magnitudes be taken from unequals, or unequals from equals, the remainders are unequal.

6. Doubles of the same magnitude, or of equal magnitudes, are equal; and doubles of unequals are unequal. 
7. Halves of the same magnitude, or of equal magnitudes, are equal; and halves of unequals are unequal.

8. The whole of anything is greater than a part of the same thing.

9. If one magnitude is greater than a second, and the second greater than a third, then the first is greater than the third.

29. A theorem is a statement of fact which is usually not self-evident, but which needs to be demonstrated before its truth becomes apparent.

For example,

(1) If two adjacent angles are supplementary, their outer boundaries lie in the same straight line. Art. 22.

(2) The sum of any two angles of a triangle is less than two right angles.

A theorem, the truth of which is self-evident, has already been called an axiom.

30. To demonstrate a theorem is to establish its truth by a process of reasoning in which reference is made only to the accepted definitions and axioms, and to the facts contained in previously established theorems.

When the truth of a theorem has once been established, the theorem is used without further question whenever it will be serviceable in the demonstration of other theorems.

31. A problem is a task to be accomplished by the use of the ruler and compasses.

For example,

(1) To draw a straight line which will divide a given angle into two equal parts.

(2) To construct a triangle whose sides shall be equal to three given line-segments.

The general term Proposition is used to include both theorems and problems. 


\section{EXERCISES}

1. Using only the ruler and compasses, draw a line-segment which shall be (1) twice as long as a given line-segment, (2) three times as long as a given line-segment.

2. Draw a line-segment equal in length (1) to the sum of two given line-segments, (2) to the difference of two given line-segments.

3. Describe a circle whose radius is (1) equal to a given line-segment, (2) double of a given line-segment.

4. Describe two circles with the same centre such that the radius of one is equal to the diameter of the other.

Definition. Circles having the same centre and unequal radii are called concentric circles.

5. Describe two circles so situated that a radius of one is a diameter of the other.

6. Describe two circles so that the same line-segment is a radius of each, but so situated that the circles do not coincide.

7. Express the supplement of (1) half a right angle, (2) two-thirds of a right angle, in terms of a right angle.

8. Draw three straight lines in a plane so that they do not all pass through one point and each intersects the other two. How many points of intersection are there?

9. Choose three points in a plane, not in the same straight line, and join each point to both the others. How many straight lines are there?

10. Draw four straight lines in a plane so that no three pass through the same point and each intersects all the others. How many points of intersection are there?

Name the lines $a, b, c, d$, and mark the points of intersection with capital letters, $A, B, C$, etc.

Point out and name the vertices of the triangles whose sides are any three of the four lines. How many such triangles are there?

To how many of these triangles does each line belong?

To how many of the triangles does each vertex belong?

11. Choose four points in a plane so that no three lie in the same straight line, and join each point to all the others. How many lines are thus drawn? Name the points $A, B, C, D$, and the lines $a, b$, etc.

Point out and name the sides of all the triangles whose vertices ar any three of the four points. How many such triangles are there?

To how many of these triangles does each vertex belong?

To how many of these triangles does each line belong? 
1. Simple Elements.

\section{SUMMARY}

Points, Lines, Surfaces.

2. Properties of the Simple Elements.

(1) Point - has no magnitude or extension. $\S 1$. has position only. $\S 1$.

(2) Line - has extension in one way, viz. length. § 2. path of a moving point. $\S 2$. contains an unlimited number of points. $\$ 2$.

(3) Surface - has extension in two ways, viz. length and breadth. $\$ 7$. contains an unlimited number of points and lines. $\S 7$.

3. Definitions.

(1) Straight Line - a line such that only one of the kind can pass through two points. $\S 4$.

(2) Plane Surface or Plane - a surface such that every straight line

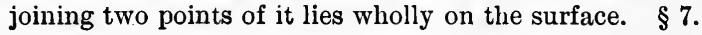

(3) Geometrical Figure - any combination of points, lines, and surfaces. $\$ 9$.

(4) Equal Geometrical Figures - two figures which can be made to occupy identically the same position. $§ 14$.

(5) Line-segment - the portion of a straight line which lies between two fixed points. $\S 5$.

Sum or Difference of Two Line-segments - see $\S 6$.

(6) Angle - the inclination of two straight lines which meet. $\$ 11$.

Boundaries of the Angle - the two straight lines forming it.

Vertex of the Angle - their common point.

Sum or Difference of Two Angles - see $\S 12$.

(7) Adjacent Angles - two angles which have a common vertex and one common boundary, without overlapping. $\$ 12$.

(8) Vertically Opposite Angles or Vertical Angles - two angles which have a common vertex, the boundaries of each being the extensions of the boundaries of the other. $\S 13$.

(9) Right Angle - an angle formed by one straight line meeting another when the two adjacent angles formed by them are equal. $\S 15$.

Acute Angle, Obtuse Angle, Reflex Angle - see §§ 19 and 20.

(10) Supplementary Angles - two angles whose sum is equal to two right angles. $\$ 19$.

(11) Straight Angle - an angle whose boundaries extend in opposite ways from the vertex and lie in the same straight line. $§ 20$. 
(12) Perpendicular Lines - lines which form a right angle. $\$ 15$.

(13) Triangle - a geometrical figure consisting of three straight lines intersecting, two and two, in three points. $\$ 10$.

Vertices of a Triangle - the points in which the three lines intersect. $\S 10$.

Sides of a Triangle - the three lines, the line-seginents between the vertices. $\S 10$.

Angles of a Triangle - the angles formed by the sides, two and two. $\S 11$.

(14) Circle - a closed line so drawn that all points of it are equally distant from a certain fixed point within it. $\$ 25$.

Centre of the Circle - the point from which all of its points are equally distant. $\$ 25$.

Radius of a Circle - a line-segment drawn from the centre to any point of the circle. $\$ 25$.

Diameter of a Circle - a line-segment drawn through the centre and terminated both ways by the circle. $\S 25$.

(15) Concentric Circles - those having the same centre and unequal radii. Ex. 4, p. 18.

(16) Postulate - a fundamental geometrical property, or construction, which is assumed. $\S 27$.

(17) Axiom - a statement the truth of which is admitted as soon as its meaning is understood. $\$ 28$.

(18) Theorem - a statement of fact which is usually not self-evident, but which needs to be demonstrated before its truth becomes apparent. $\S 29$.

(19) Problem - a task to be accomplished by the use of the ruler and compasses. $\S 31$.

4. Postulates.

See $\S 27$ for Postulates 1-4.

5. Axions.

See $\S 28$ for Axioms 1-9.

6. Theorems.

(1) The sum of the two angles which one straight line makes with another on one side of it is equal to two right angles; and when two straight lines intersect, the sum of the four angles formed at their point of intersection is equal to four right angles. $§ 16$. 
(2) If from a point in a straight line any number of straight lines are drawn in a plane on one side of the line, the sum of all the angles so formed is equal to two right angles. $\S 18$.

(3) If from a point any number of straight lines are drawn in a plane, the sum of all the angles so formed is equal to four right angles. $\S 18$.

(4) All straight angles are equal. $§ 21$.

(5) All right angles are equal. $\$ 21$.

(6) If two angles are equal, their supplements are equal, and two different supplements of the same angle are equal. $\$ 21$.

(7) If two adjacent angles are supplementary, their outer boundaries lie in the same straight line. $\$ 22$.

7. On the Use of Instruments.

See $\S 26$. 


\section{CHAPTER I}

\section{TRIANGLES AND PARALLELOGRAMS}

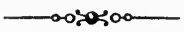

\section{SECTION I}

\section{GENERAL PROPERTIES OF TRIANGLES}

32. After laying so much of a foundation we are ready to proceed with the task of building up the main body of geometrical knowledge, and it must be constantly borne in mind that this subject is a continuous development. We shall meet new truths at every turn, and every new truth will aid us in the establishment of still other new truths.

We shall first present a few easy problems connected with the construction of triangles.

\section{Definitions}

33. A triangle whose three sides are unequal in length is called a scalene triangle.

A triangle which has two equal sides is called an isosceles triangle.

A triangle whose three sides are all equal is called an equilateral triangle.

Is an equilateral triangle also isosceles?

Is an isosceles triangle ever equilateral? 


\section{Proposition I}

34. It is required to construct an equilateral triangle of which a given line-segment is one side.

First, recall what an equilateral triangle is. If one side is given in length, how long are the other two sides?

Now state the proposition in specific terms, thus :

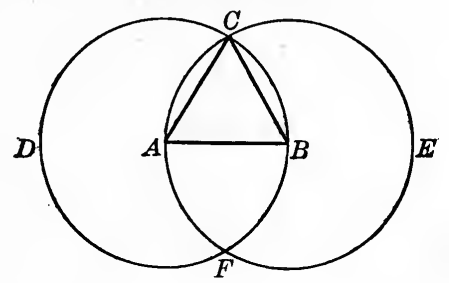

Let $A B$ (see the diagram) be the given line-segment.

It is required to construct an equilateral triangle, of which $A B$ is one side.

Next proceed with the construction.

With centre $A$, and radius $A B$, describe a circle.

With centre $B$, and radius $B A$, describe a circle.

These circles will intersect in two points. Why? (See. Art. 25.)

Let $C$ be one of their points of intersection.

Join $A C$ and $B C$.

Note. When we say 'Join $A C$,' we mean draw a straight line from the point $A$ to the point $C$.

The triangle $A C B$ so constructed has the line-segment $A B$ for one side; but it remains to show that this triangle is equilateral.

Proof. Because $A$ is the centre of the first circle, and the line-segments $A B$ and $A C$ are radii, therefore $A C$ equals $A B$. 
Because $B$ is the centre of the other circle, and the linesegments $B A$ and $B C$ are radii, therefore $B C^{\prime}$ equals $B A$.

That is, $A C$ and $B C$ are each equal to $A B$.

Hence $A C$ equals $B C$.

(Axiom 1)

Therefore $A B, A C, B C$ are all equal, and the triangle $A B C$ is equilateral.

\section{EXERCISES}

1. Is this proposition a problem or a theorem?

2. If $F$ is the other point of intersection of the two circles, and we join $A F$ and $B F$, prove that $A F B$ is also an equilateral triangle.

3. Join $C F$. Show that the triangles $A C F$ and $B C F$ are each isosceles.

35. The student should carefully note the order of arrangement in the preceding proposition. The same order, written out in less detail, perhaps, will be followed in all the propositions. We have

1. The general enunciation of the proposition; that is, the statement of the proposition in general terms, and the student, after reading the enunciation, should proceed no further until he thoroughly understands just what he is expected to do.

2. A re-statement of the proposition applied to a particular figure, or what is sometimes called the particular enunciation.

3. Making the necessary construction.

4. Proof that the thing constructed is what was required. In the case of a theorem the proof goes to show that the statement made in the enunciation is true.

36. In naming a circle we usually mention three points on it, these being sufficient to distinguish it from every other circle.

Thus, in the diagram of this proposition, the circle whose centre is $A$ would be called the circle $B C D$, and the circle whose centre is $B$ would be called the circle $A C E$.

Exercise. Would naming its centre and one point on it be sufficient to distinguish a circle from all other circles? 


\section{Proposition II}

[General Enunciation]

37. To construct a triangle two of whose sides shall be each equal to one given line-segment, and the third side equal to another given line-segment.

What sort of a triangle will this be ?

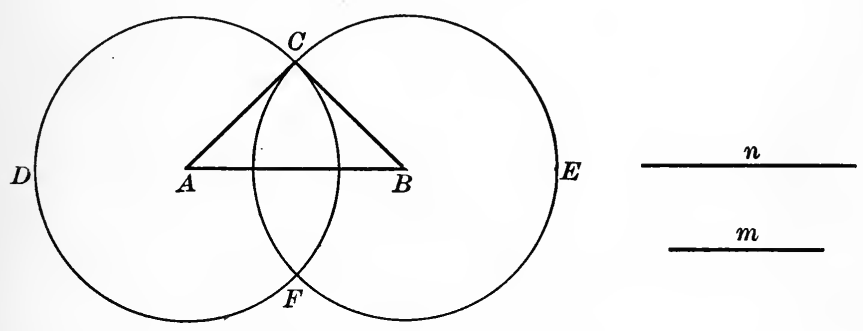

\section{[Particular Enunciation]}

Let $m$ and $n$ be the given line-segments.

It is required to construct a triangle, of which two sides are each equal to $m$ and the third side equal to $n$.

\section{[Construction]}

Draw anywhere in the plane a line-segment $A B$ equal to $n$. With centre $A$, and radius equal to $m$, describe a circle $C D F$.

With centre $B$, and radius also equal to $m$, describe a circle CEF.

Suppose these two circles intersect at $C$.

Do these circles necessarily intersect? If so, at how many points?

Join $C A$ and $C B$.

Then $C A B$ is the triangle required. 


\section{[Proof $]$}

Let the pupil make a diagram for himself and letter it as in the construction.

In the triangle $C A B$, the side $A C$ is a radius of the circle $C D F$, hence $A C$ equals $m$.

All radii of a circle are equal by definition. (See Art. 25.)

The side $B C$ is a radius of the circle $C E F$, hence $B C$ equals $m$. Explain why.

The side $A B$ was constructed equal to $n$.

Hence the triangle $C A B$ fulfils the given conditions, having two sides equal to $m$, and one side equal to $n$.

\section{EXERCISES}

1. Do the two circles in the above construction necessarily intersect? Suppose the line $n$ were more than twice as long as $m$, what then? Can a triangle be formed with sides 6 feet, 6 feet, and 13 feet in length ?

2. If the line-segments $m$ and $n$ are equal, what sort of triangle does $C A B$ become?

3. Construct a triangle two of whose sides shall be each equal to the longer line-segment $n$, and the third side equal to the shorter linesegment $m$.

4. If $A F, B F$, and $C F$ are joined, show that $A C F$ and $B C F$ are each isosceles triangles.

5. Show how to find a point equidistant from two given points, making use only of the compasses.

38. For convenience, one side of a triangle is sometimes called the base of the triangle, and the other two, the sides. The base may be any side whatever; the opposite angle is then called the vertical angle, and the vertex of that angle, the vertex of the triangle. The two angles adjacent to the base are called the base angles.

In an isosceles triangle it is usually the unequal side which is called the base. 


\section{Proposition III}

39. To construct a triangle having its sides respectively equal to three given line-segments.

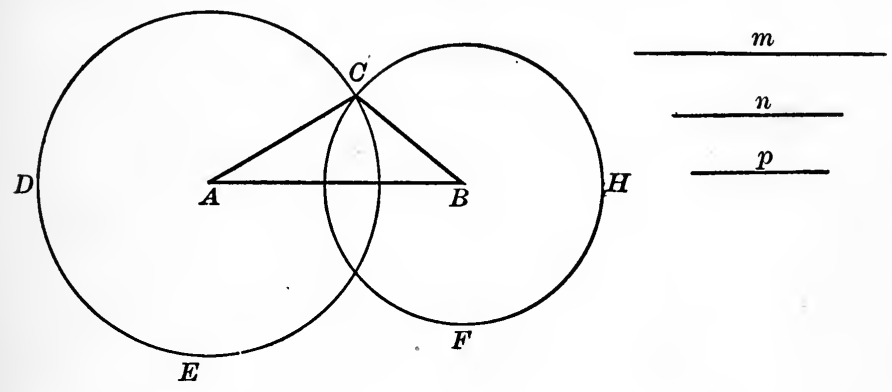

Let $m, n, p$, be the three given line-segments.

It is required to construct a triangle whose sides are respectively equal to $m$, $n$, and $p$.

Construction. Draw a line-segment $A B$ anywhere in the plane equal, say, to $m$, the longest of the three given line-segments.

With centre $A$, and radius equal to $n$, describe a circle $C D E$.

With centre $B$, and radius equal to $p$, describe a circle CHF.

Suppose these two circles intersect at $C$.

Join $C A$ and $C B$.

The triangle $C A B$ is the triangle required.

Proof. In the triangle $C A B$, the side $C A$ equals the given line-segment $n$ [why?]; the side $C B$ equals the given linesegment $p$ [why?]; and the side $A B$ was chosen equal to the given line-segment $m$.

Hence the triangle $C A B$ fulfils the given conditions. 


\section{EXERCISES}

1. Under what conditions would the circle $C D E$ pass through the point $B$, or the circle $C H F$ through the point $A$ ?

2. Under what conditions would the two circles $C D E$ and $C H F$ not intersect? Could the required triangle be constructed in that case ?

3. If the line $m$ is greater than the sum of $n$ and $p$, see what the diagram would look like if, instead of choosing the first line equal to $m$, you should choose it equal to $n$ or $p$.

4. Can you infer from this solution that one side of a triangle cannot be greater than the sum of the other two sides? Keep this in mind for future consideration.

40. If two triangles are identically equal, either of them can be made to occupy the position of the other (Art. 14); or, in other words, the one can be placed on the other so that their vertices will coincide and their sides coincide. In this case the three sides of either triangle must be equal, respectively, to the three sides of the other, and the three angles of either, equal respectively to the three angles of the other, and the two triangles are said to be equal in all respects.

But the question arises immediately, If two triangles are equal in some respects, is not this sufficient to make them equal in other respects, or perhaps in all respects?

In other words, if two triangles can be made to coincide in some of their parts, will they not necessarily coincide in all their parts?

We speak of the three sides and the three angles of a triangle as the parts of the triangle.

The next two or three propositions will relate to questions of this kind.

The method of testing the equality of two geometrical figures by placing the one on the other is known as the method of superposition. 


\section{Proposition IV}

41. If two triangles have two sides and the included angle of the one equal, respectively, to two sides and the included angle of the other, the triangles are identically equal.
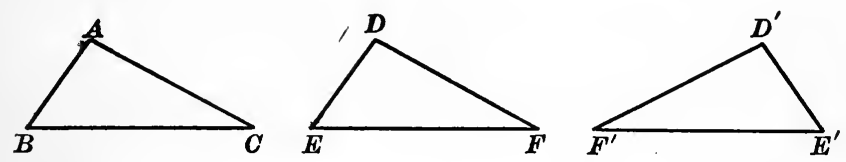

Let $A B C$ and $D E F$ be the two given triangles in which the side $A B$ equals the side $D E$, the side $A C$ equals the side $D F$, and the angle $B A C$ equals the angle $E D F$.

It is required to prove that the third side $B C$ equals the third side $E F$, that the angle $A B C$ equals the angle $D E F$, and that the angle $A C B$ equals the angle $D F E$.

Proof. Place the triangle $D E F$ upon the triangle $A B C$ so that the vertex $D$ shall coincide with the vertex $A$, and the side $D E$ shall fall on its equal side $A B$.

Then because $D E$ equals $A B, E$ must coincide with $B$. Since the angle $E D F$ equals the angle $B A C$, and $D E$ coincides with $A B, D F$ will fall on $A C$.

It may be necessary to turn the triangle over in order to make $D F$ fall on $A C$, as would be the case with the triangle $D^{\prime} E^{\prime} F^{\prime}$, but this is allowable. (See Postulate 4.)

And because $D F$ equals $A C, F$ must coincide with $C$.

Call to mind at this point what three parts of one triangle were given equal to three parts of the other.

Now, two points $E$ and $F$ of the straight line $E F$ have been made to coincide, respectively, with two points $B$ and $C$ of the straight line $B C$. 
Hence these two straight lines must coincide throughout.

If two straight lines coincide in two points, they coincide at every point. (Art. 4.)

And the line-segment $E F$ must equal the line-segment $B C$.

Therefore, since the two triangles $A B C$ and $D E F$ have been made to coincide in all their vertices and all their sides, their angles must be equal, respectively, and the two triangles must be identically equal.

Notice particularly that in these two triangles the angles which are equal are opposite sides which are equal.

Such angles or sides are said to be similarly situated in the two triangles. They are sometimes called corresponding angles or sides; or homologous angles or sides.

42. In a theorem there are always two distinct parts. It reads :

If certain things are true, then certain other things are true. The first part, the if part, is called the hypothesis. The second part, the then part, is called the conclusion.

The theorem of Proposition IV may be divided into hypothesis and conclusion, thus:

Hypothesis. If two triangles have two sides and the included angle of the one equal, respectively, to two sides and the included angle of the other,

Conclusion. Then the triangles are identically equal.

Separate the next proposition into hypothesis and conclusion.

\section{EXERCISES}

In the two triangles $A B C$ and $D E F$,

1. If $A B$ equals $D E$, and $A C$ equals $D F$, but the angle $B A C$ is greater than the angle $E D F$, where would $D F$ fall when the, triangle $D E F$ is placed upon the triangle $A B C$ as in this proposition?

2. If the angles were equal, but $A C$ greater than $D F$, where would $F$ fall?

3. Is Proposition IV a problem or a theorem? 


\section{Proposition V}

43. If two triangles have a side and the two adjacent angles of the one equal, respectively, to a side and the two adjacent angles of the other, the triangles are identically equal.*
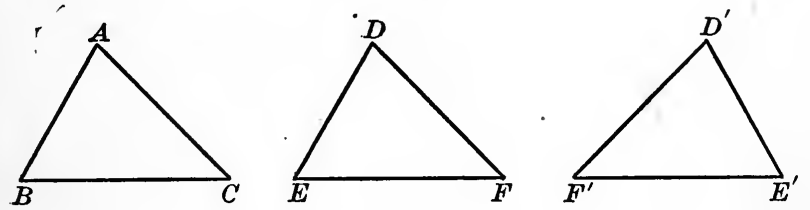

This proposition, like the last one, is proved by superposition.

Let $A B C$ and $D E F$ (or $D^{\prime} E^{\prime} F^{\prime}$ ) be two given triangles in which the side $E F$ is equal to the side $B C$, and the angles at $E$ and $F$ are respectively equal to the angles at $B$ and $C$.

It is required to prove that the two triangles are identically equal.

Proof. Place the triangle $D E F$ upon the triangle $A B C$ so that the side $E F$ shall coincide with its equal side $B C$, the point $E$ coinciding with $B$, and $F$ with $C$, and so that the vertices $D$ and $A$ lie on the same side of $B C$.

Then, since the angle $F E D$ equals the angle $C B A$, the side $E D$ must fall on $B A$, and the point $D$ will coincide with some point of the line $B A$.

Why do we not know at this stage that $D$ coincides with $A$ ?

Also, since the angle $E F D$ equals the angle $B C A$, the side $F D$ must fall on the side $C A$, and the point $D$ will coincide with some point of the line $C A$.

* It should be observed that by 'the two adjacent angles' we mean the two angles adjacent to this side, or of which this side is a common boundary. Do not confuse this with 'adjacent angles' defined on p. 8. 
Since $D$ coincides both with some point of $B A$ and with some point of $C A$, it must coincide with their point of intersection, namely, with $A$.

Therefore, since the two triangles have been made to coincide in all their vertices and all their sides, they are identically equal.

44. It will be interesting to notice the relation between the last two propositions.

Proposition IV may be stated:

Hy ротнеsis. If two triangles have two sides and the included angle of the one equal, respectively, to two sides and the included angle of the other,

Corclusios. Then the base and the two adjacent angles of the one are equal, respectively, to the base and the two adjacent angles of the other.

\section{Proposition V may be stated:}

Hypotiesis. If two triangles have the base and the two adjacent angles of the one equal, respectively, to the base and the two adjacent angles of the other,

Conclusion. Then the two sides and the included angle of the one are equal, respectively, to the two sides and the included angle of the other.

Stated in this way, the hypothesis in Proposition IV becomes the conclusion in Proposition V, and the conclusion in Proposition IV, the hypothesis in Proposition V.

Two theorems so related are said to be converse.

Definition. Two theorems are converse when the hypothesis of each is the conclusion of the other.

\section{Notation}

45. Hereafter, whenever it is desirable, we shall make use of certain symbols to abbreviate our forms of statement. For instance, instead of writing in full 'the angle $A B C$,' we shall write $\angle A B C$; instead of 'the angle at $B$,' we shall write $\angle B$; instead of 'the triangle $H K L$,' we shall write $\triangle H K L$; and instead of 'is equal to,' or 'equals,' we shall sometimes use the 
algebraic symbol $=$. But care should be taken in every case to read the symbol as though the statement were written out in full.

\section{ON Bisectors}

46. If through the vertex of an angle $B A C$, we draw any straight line $A D$, it divides the angle into two parts, $B A D$ and $D A C$. These two parts may be equal, or they may be unequal. If they are equal, the line $A D$ is said to bisect the angle $B A C$.

If the angles $B A D$ and $D A C$ are unequal, suppose that $B A D$ is smaller than $D A C$, and rotate $A D$ in the way indicated by the arrowhead. This

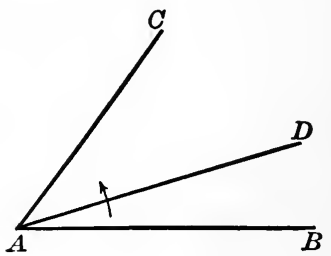
will continuously increase the one angle and continuously decrease the other, while it leaves their sum unaltered.

It is clear that there must be some position of $A D$ for which the angles $B A D$ and $D A C$ are equal; and that if $A D$ is noved ever so little from that position, one of these angles will be increased while the other is diminished, so that they will become unequal.

Hence,

Through the vertex of any angle there can be drawn one, and only one, straight line which bisects the angle.

If the angle bisected is a straight angle (see Art. 20), the bisector is perpendicular to the straight line which bounds the angle. From this it follows immediately that

At any point in a straight line there can be drawn one, and only one, perpendicular to the line.

47. Just as we are able to show that there is one, and only one, 'bisector' of an angle, so we might show that there is one, and only one, 'point of bisection' or 'mid-point' of a linesegment. 


\section{Proposition VI}

48. If two sides of a triangle are equal, the angles opposite those sides are also equal.

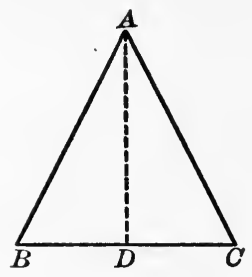

Let $A B C$ be an isosceles triangle, $B A$ and $C A$ being the equal sides.

It is required to prove that the angle at $C$, opposite the side $B A$, equals the angle at $B$, opposite the side $C A$.

Proof. Suppose that the line $A D$ is the bisector of $\angle B A C$, and that it meets $B C$ at $D$. It thus divides $\triangle B A C$ into two triangles ; namely, $\triangle B A D$ and $\triangle C A D$.

In these two triangles, the sides $B A$ and $A D$ are equal, respectively, to the sides $C A$ and $A D$, and the included angle $B A D$ equals the included angle $C A D$.

Hence $\triangle \mathrm{s} B A D$ and $C A D$ are equal in all respects.

(Prop. IV.)

Therefore $\angle B$ equals $\angle C$, these being corresponding angles, i.e. opposite equal sides, in the two triangles.

49. The proof of this proposition contains the proof of the following theorem:

The straight line which bisects the vertical angle of an isosceles triangle also bisects the base, and is perpendicular to the base.

For since $\triangle \mathrm{s} B A D$ and $C A D$ are identically equal, $B D=C D$ and $\angle B D A=\angle C D A$. Therefore $B C$ is bisected at the point $D$, and $A D$ is perpendicular to $B C$. 
50. Definition. A theorem, the truth of which is easily deduced from another, or the proof of which is easily obtained from the proof of another, is said to be a corollary of that other.

The following is a second corollary to Proposition VI:

Corollary. An equilateral triangle is also equiangular.

The pupil should show that if the three sides of a triangle are equal, the three angles are equal.

While the proof of a corollary is easily deduced from that of the main proposition, it should never be neglected, but should in most cases be carefully written out by the pupil.

\section{EXERCISES}

1. If two isosceles triangles, $A B C$ and $D B C$, are on the same base, $B C$, but on opposite sides of it, prove (1) that the angle $A B D$ is equal to the angle $A C D$, (2) that if $A D$ be drawn $\triangle A B D$ is identically equal to $\triangle A C D,(3)$ that $A D$ bisects the vertical angle of each triangle, (4) that $A D$ bisects the common base of the triangles, (5) that $A D$ makes right angles with the base.

2. Prove the same things when the two triangles lie on the same side of the base.

3. If the vertex of an isosceles triangle is joined to the mid-point of the base, prove that the line so drawn bisects the vertical angle of the triangle, and also that it is perpendicular to the base.

4. On a given line-segment construct two different isosceles triangles, and make use of these to find the mid-point of the given line-segment.

5. Prove that the triangle whose vertices are the mid-points of the sides of an equilateral triangle is equilateral. (Apply Prop. IV.)

6. Prove that the triangle whose vertices are the mid-points of the sides of an isosceles triangle is isosceles. (Apply Prop. IV.)

7. If on the sides $A B, B C, C A$, of an equilateral triangle $A B C$, equal lengths $A P, B Q, C R$, are taken, prove that the triangle $P Q R$ is also equilateral. 


\section{Proposition VII}

51. If two angles of a triangle are equal, the sides opposite those angles are also equal.

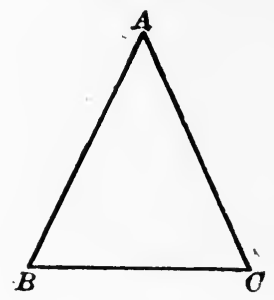

Let $A B C$ be a triangle in which the angle $A B C$ equals the angle $A C B$.

It is required to prove that the side $C A$ is equal to the side $B A$.

Proof. Mark the positions of the vertices and sides of the triangle, and then suppose the triangle to be turned over and put down so that the vertex $B$ takes the former position of the vertex $C$, and the vertex $C$ the former position of the vertex $B$.

The side $B C$ will thus occupy its former position, but will be turned end for end.

Since $\angle B$ equals $\angle C$, the side $B A$, when the triangle is turned over, will fall upon the former position of the side $C A$, and the side $C A$ will fall upon the former position of the side $B A$.

Therefore the point $A$ will occupy its former position, and the side $B A$ will coincide with the former position of the side $C A$.

Therefore the side $B A$ equals the side $C A$.

52. Corollary. An equiangular triangle is also equilateral.

The pupil should prove that if the three angles of a triangle are equal, the three sides must be equal. 


\section{Proposition VIII}

53. If two triangles have the three sides of the one equal, respectively, to the three sides of the other, the two triangles are identically equal.
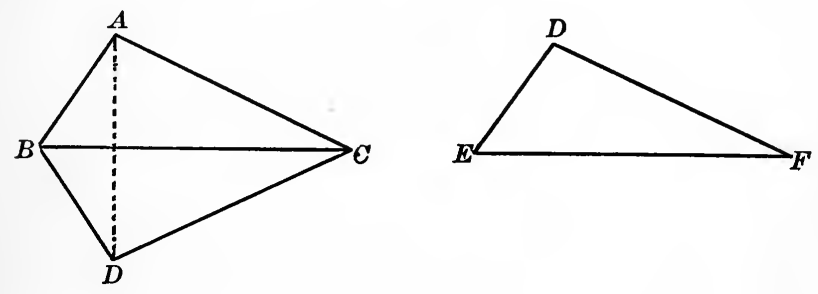

Let $A B C$ and $D E F$ be two triangles having the side $A B$ equal to the side $D E, B C$ equal to $E F$, and $C A$ equal to $F D$.

It is required to prove that the two triangles are identically equal.

Proof. Place the triangle $D E F$ so that the side $E F$ will coincide with its equal side $B C$, the point $E$ with $B$, and the point $F$ with $C$, and so that the vertex $D$ will fall on the opposite side of $B C$ from the vertex $A$.

Join $A D$.

Then in $\triangle A B D, \angle B A D=\angle B D A$,

(Prop. VI.)

and in

$$
\triangle A C D, \angle C A D=\angle C D A \text {. }
$$

Why?

Therefore

$$
\angle B A C=\angle B D C \text {. }
$$

But $\angle B D C$ is $\angle E D F$, which is therefore equal to $\angle B A C$.

Hence in $\triangle \mathrm{s} B A C$ and $E D F$, two sides and the included angle of one are equal, respectively, to two sides and the included angle of the other: .Name them.

Therefore the two triangles are identically equal. (Prop. IV.) 
In Article 46 we showed that for every angle there is one and only one bisector, and also stated in Art. 47 that for every line-segment there is one and only one mid-point, or bisecting point. In the next two propositions, we shall give simple methods for finding these bisectors with the ruler and compasses.

\section{Proposition IX}

54. To bisect a given angle.
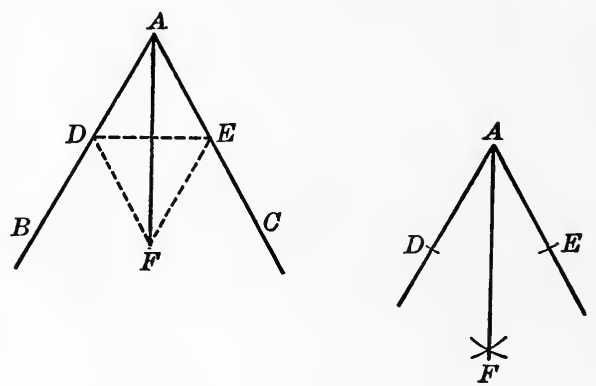

Let $B A C$ be a given angle.

It is required to draw the straight line which will bisect it.

Construction. From $A B$ and $A C$ cut off any equal segments $A D$ and $A E$. [Use compasses.]

Join $D E$.

Upon $D E$ as base construct any isosceles triangle having its vertex $F$ on the opposite side of $D E$ from $A$.

(Prop. II.)

Join $A F$.

Then $A F$ is the required line.

Proof. Compare $\triangle \mathrm{s} A D F$ and $A E F$ side for side.

These triangles are equal in all respects. Where proved?

Therefore $\angle D A F$ equals $\angle E A F$. That is $\angle B A C$ is bisected by $A F$. 
55. It should be noticed that in order to draw the bisector $A F$, it is not necessary to draw the straight lines $D E, D F$, and $E F$. The points $D, E$, and $F$ can be located with the compasses, and then the line $A F$ drawn, and in actual practice this is all that should be done. The other lines, however, are necessary for the proof.

\section{EXERCISES}

1. If $A B C$ is an isosceles triangle, and the equal angles $A B C$ and $A C B$ are bisected by the lines $B D$ and $C D$ which meet at $D$, prove that $D B C$ is also an isosceles triangle.

2. $A B C$ is an isosceles triangle having $A B$ equal to $A C$. In $A B$ and $A C$ two points $D$ and $E$ are taken equally distant from $A$; prove that the line-segments $B E$ and $C D$ are equal.

(Prop. IV.)

3. If two line-segments bisect each other at right angles, prove that any point in one of them is equidistant from the extremities of the other.

4. $B A C$ is a triangle having the angle $B$ double of the angle $A$; if $B D$ bisects the angle $B$ and meets $A C$ at $D$, prove that $B D$ equals $A D$.

5. $A C B$ and $A D B$ are two triangles on the same side of $A B$, such that $A C$ is equal to $B D$, and $A D$ is equal to $B C, A C$ and $B D$ intersecting at $O$. Show that the triangle $A O B$ is isosceles.

6. Divide a given angle into four equal parts.

7. If in a triangle $D E F$ the angles $D$ and $E$ are bisected by straight lines meeting at $H$, and $D H$ equals $E H$, prove that $D F$ equals $E F$.

8. Prove that the bisectors of the angles of an equilateral triangle meet in one point. Is the same thing true of an isosceles triangle?

Suggestion. Draw the bisectors of two equal angles and let them meet at $O$. Join $O$ to the third vertex, and show that the line so drawn bisects the third angle.

9. Apply the construction of Proposition IX to bisect a straight angle, i.e. to draw a perpendicular to a given straight line at a given point.

10. If $D$ and $E$ are the mid-points of the equal sides $A C$ and $A B$ of an isosceles triangle $A B C$, prove that $B D$ equals $C E$.

11. Let $D$ be any point on the bisector of an angle $B A C$; prove that if $A B$ equals $A C$, the angle $A D B$ equals the angle $A D C$. 


\section{Proposition X}

56. To bisect a given line-segment.

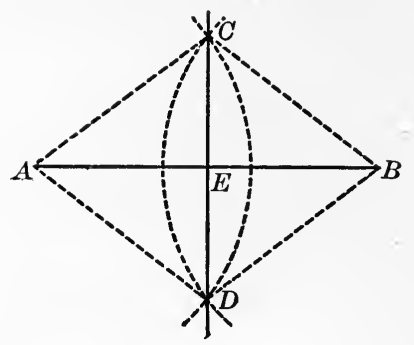

Let $A B$ be a given line-segment, which it is required to bisect.

Construction. With centre $A$ and any convenient radius describe a circle.

With centre $B$, and an equal radius, describe another circle.

Let these circles intersect at points $C$ and $D$.

Note. The radius must be chosen long enough to make the two circles intersect.

Draw the straight line $C D$, meeting $A B$ at $E$.

Then $E$ is the mid-point of the line-segment $A B$.

Proof. Join $C A$ and $C B$, also $D A$ and $D B$.

Since these line-segments are all equal, $\triangle \mathrm{s} A C D$ and $B C D$ are identically equal.

(Prop. VIII.)

Therefore

$$
\angle A C D=\angle B C D .
$$

In the triangles $A C E$ and $B C E$, two sides and the included angle in one are equal to two sides and the included angle in the other.

Therefore these triangles are identically equal. (Prop. IV.) And the line-segment $A B$ is bisected at the point $E$. 
Since $C D$ not only bisects $A B$ at $E$, but is also perpendicular to $A B$, it may be called the perpendicular bisector of $A B$.

57. Corollary. Two points each equidistant from the extremities of a given line-segment determine the perpendicular bisector of the segment.

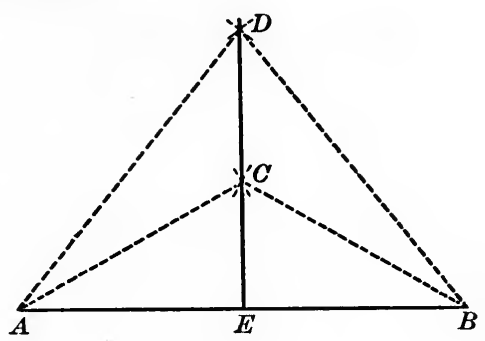

FIG. 1.

The figure of the proposition illustrates one case of the theorem stated in the corollary. Another case arises witen $C$ is equidistant from $A$ and $B$, and also $D$ equidistant from $A$ and $B$, as in Fig. 1 .

Suggestion. It is here required to prove that $D C$ bisects $A B$ at right angles. From $\triangle \mathrm{s} A D C$ and $B D C$ prove $\angle A D C=\angle B D C$.

(Prop. VIII.)

From $\triangle \mathrm{s} A D E$ and $B D E$ prove $A E=B E$ and $\angle A E D=\angle B E D$.

(Prop. IV.)

\section{EXERCISES}

1. What lines of the figure of Proposition $\mathrm{X}$ were necessary to find the point $E$, and what lines had to be added for the proof?

2. In the figure of Proposition $\mathrm{X}$ show that $C D$ is also bisected at right angles by $A B$.

3. If two circles intersect, the straight line joining their centres bisects at right angles the line-segment joining their points of intersection.

4. Find a line-segment equal to (1) half the sum of two given linesegments, (2) half the difference of two given line-segments.

5. Find a line-segment half as long again as a given line-segment.

6. Prove that every point on the perpendicular bisector of a linesegment is equidistant from the extremities of the line-segment. 


\section{Proposition XI}

58. If two straight lines intersect, the vertical angles are equal.

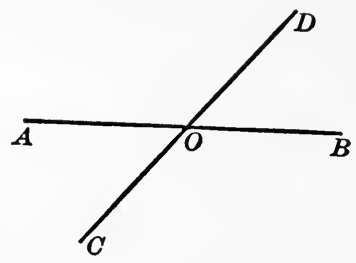

Let the straight lines $A B$ and $C D$ intersect at $O$.

It is required to prove that the vertical angles $A O C$ and $B O D$ are equal.

Proof. $\angle \mathrm{s} A O C$ and $A O D$ are together equal to two right angles.

Hence $\angle A O C$ is the supplement of $\angle A O D$.

(Art. 16.)

The $\angle \mathrm{s} B O D$ and $A O D$ are together equal to two right angles. Why?

Hence $\angle B O D$ is the supplement of $\angle A O D$.

Therefore $\angle A O C$ and $\angle B O D$, being supplements of the same angle, are equal.

(Art. 21.)

Similarly it may be shown that the vertical angles $A O D$ and $B O C$ are equal.

\section{EXERCISES}

1. The straight line which bisects one of two vertical angles bisects also the other.

2. The straight lines which bisect two adjacent supplementary angles are at right angles to each other.

3. If one straight line is perpendicular to a second, the second is perpendicular to the first.

In connection with this example you will need carefully to recall the definition of a perpendicular given in Art. 15.

4. If one of the four angles made by two intersecting straight lines is a right angle, the other three are also right angles. 


\section{Proposition XII}

59. If any side of a triangle is produced, the exterior angle so formed is greater than either of the two interior non-adjacent angles.

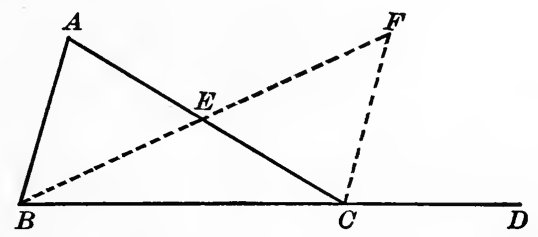

Let the triangle $A B C$ have the side $B C$ produced to $D$.

It is required to prove that the angle $A C D$ is greater than either the angle at $A$ or the angle at $B$.

Proof. Bisect the side $A C$ at $E$, join $B E$, and produce to $F$, making $E F$ equal to $B E$. Join $F C$.

$\triangle E F C$ is identically equal to $\triangle E B A$. Why?

Apply Propositions XI and IV.

Therefore $\angle E C F$ equals its corresponding $\angle E A B$.

But $\angle A C D$ is greater than $\angle E C F$. Therefore $\angle A C D$ is greater than $\angle C A B$, or $\angle A$.

To prove $\angle A C D$ greater than $\angle B$, it is only necessary to produce the side $A C$, thus forming a vertical angle equal to $\angle A C D$, then proceed just as before, bisecting the side $B C$ instead of the side $A C$.

\section{EXERCISES}

1. Show that $\angle A$ is less than $\angle A E F$, and that $\angle F$ is less than $\angle B E C$.

2. Draw three figures to show that an exterior angle of a triangle may be greater than, equal to, or less than the interior adjacent angle. 


\section{Proposition XIII}

60. Any two angles of a triangle are together less than two right angles.

Suggestions for Proof. Produce one side of the triangle and so form an exterior angle.

This exterior angle is greater than either of the interior nonadjacent angles.

(Prop. XII.)

The sum of the interior adjacent angle and this exterior angle is equal to two right angles.

(Art. 16.)

Therefore the sum of the interior adjacent angle and either of the other interior angles is less than two right angles.

By producing a different side of the triangle the angles can be taken in pairs in other ways, and the theorem can thus be proved for all possible pairs.

The pupil should make his own diagram and write out the proof in full, naming the angles used.

61. Corollary I. If a triangle has one right angle or one obtuse angle, the other two angles must be acute.

Definition. A triangle which has a right angle is called a right triangle.

The side opposite the right angle is called the hypotenuse.

62. Corollary II. From a point outside a given straight line, not more than one perpendicular to the line can be drawn.

\section{EXERCISES}

1. Show by joining the vertex $A$ of a triangle $A B C$ with any point of the opposite side between $B$ and $C$ that the angles at $B$ and $C$ are together less than two right angles.

2. If any side of a triangle is produced both ways, the two exterior angles so formed are together greater than two right angles.

3. The angles at the base of an isosceles triangle are both acute. 


\section{Proposition XIV}

63. If two sides of a triangle are unequal, the angle opposite the greater side is greater than the angle opposite the less.

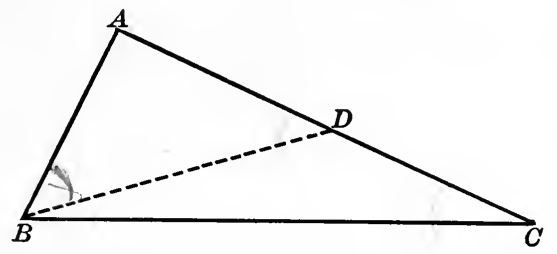

Let $A B C$ be a triangle in which the side $A C$ is greater than the side $A B$.

It is required to prove that the angle $B$ is greater than the angle $C$.

Proof. From $A C$ cut off a part $A D$ equal to $A B$. Join $B D$. The line $B D$ necessarily falls within $\angle A B C$.

In $\triangle A B D, \angle A B D$ equals $\angle A D B$. Why?

But $\angle A D B$ is greater than $\angle A C B$. Why?

Therefore $\angle A B D$ is greater than $\angle A C B$.

But $\angle A B C$ is greater than $\angle A B D$.

Therefore $\angle A B C$ is greater than $\angle A C B$.

(Axiom 8.)

(Axiom 9.)

\section{EXERCISES}

1. The angles in a scalene triangle are all unequal.

2. If one side of a triangle is less than another, the angle opposite it must be acute.

3. Prove the proposition by producing $A B$ to $D$, making $A D$ equal to $A C$ and joining $D C$.

4. If straight lines are drawn from any point within a triangle to two of the vertices of the triangle, the angle contained by these lines is greater than the angle at the third vertex.

Suggestion. Draw a line from the third vertex through the chosen point. Then compare angles. Find also a second proof. 
64. Axion 10. Two magnitudes $A$ and $B$ of the same kind are either equal, or $A$ is greater than $B$, or $A$ is less than $B$.

\section{Proposition XV}

65. If two angles of a triangle are unequal, the side opposite the greater angle is greater than the side opposite the less.

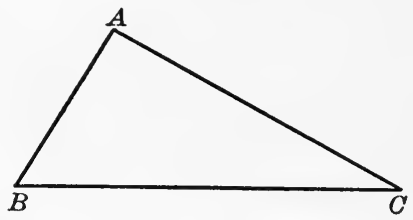

Let $A B C$ be a triangle in which the angle $B$ is greater than the angle $C$.

It is required to prove that the side $A C$ is greater than the side $A B$.

Proof. If the side $A C$ is not greater than $A B$, it must be equal to $A B$, or less than $A B$.

(Axiom 10.)

If $A C$ were equal to $A B$, how would $\angle \mathrm{s} B$ and $C$ compare? Give reference.

If $A C$ were less than $A B$, how would $\angle \mathrm{s} B$ and $C$ compare? Give reference.

Conclusion.

66. Corollary I. In a right triangle the hypotenuse is the grealest side.

67. Corollary II. Not more than two equal straight lines can be drawn from a given point to a given straight line.

Suppose a third line $A D$, equal to the first two, $A B$ and $A C$, can be drawn. Then $\angle A B C=\angle A C B, \angle A B D=\angle A D B$, and $\angle A C D=\angle A D C$. (Prop. VI.) But this is impossible by Proposition XII. 
68. The theorems of Propositions XIV and XV are converse, as are also those of Propositions VI and VII.

Proposition VIII may be stated:

If two triangles have the three sides of the one equal, respectively, to the three sides of the other, then the three angles of the one are equal, respectively, to the three angles of the other.

State the converse. Is the converse true? Can a theorem be true and its converse be false?

69. Special attention should be paid to the method of proof in Proposition XV. It is a method very frequently used when we wish to prove the converse of a theorem whose truth has already been established.

In the theorem of this proposition the statement to be proved is that one side $p$ of a triangle is greater than another side $q$, upon the hypothesis that the opposite angle $P$ is greater than the opposite angle $Q$, and we proceed as follows :

1. Suppose that the statement is not true. The only other possibilities are: First, $p$ is equal to $q$, or second, $p$ is less than $q$.

2. Show by reference to a previous theorem (Prop. VI.) that if $p$ is equal to $q$, then the hypothesis cannot be true.

3. Show also by reference to a previous theorem (Prop. XIV.) that if $p$ is less than $q$, then again the hypothesis cannot be true.

4. Conclude that since with the given hypothesis neither of the other possibilities can be true, the statement of the theorem must be true.

Care must be taken in such a proof that all possible cases have been examined before drawing a conclusion.

Such a proof is called an indirect proof.

\section{EXERCISES}

1. Prove that if $D$ be any point in the base $B C$ of an isosceles triangle $A B C$, between $B$ and $C, A D$ is less than $A B$; but if $D$ lie on the base produced either way, then $A D$ is greater than $A B$.

2. $A B C$ is a triangle in which $O B$ and $O C$ bisect the angles $B$ and $C$, respectively; show that if $A B$ is greater than $A C$, then $O B$ is greater than $O C$. 


\section{Proposition XVI}

70. Any two sides of a triangle are together greater than the third side.

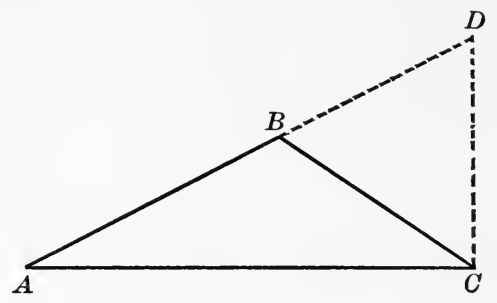

Let $A B C$ be any triangle.

It is required to prove that any two sides $A B$ and $B C$ are together greater than the third side $A C$.

Proof. Produce the side $A B$ to $D$, making $B D$ equal to $B C$. Join $D C$.

Then $A D$ is the sum of $A B$ and $B C$, and it is required to prove that $A D$ is greater than $A C$.

$\angle B C D$ equals $\angle B D C$. Why?

Therefore $\angle A C D$ is greater than $\angle B D C$, or $\angle A D C$.

Therefore in $\triangle A D C$, the side $A D$ is greater than the side $A C$. Give reference.

Hence in $\triangle A B C$, the sides $A B$ and $B C$ are together greater than the side $A C$.

\section{EXERCISES}

1. Prove this theorem by bisecting one angle and producing the bisector to meet the opposite side.

2. Could a triangle be formed whose sides are 7, 11, and 19 feet, respectively? What is the limitation on the construction in Proposition III.

3. Prove that the difference between any two sides of a triangle is less than the third side.

4. A side of an isosceles triangle is always greater than half the base. 


\section{Proposition XVII}

71. From a given point in a straight line to, draw a perpendicular to the line.

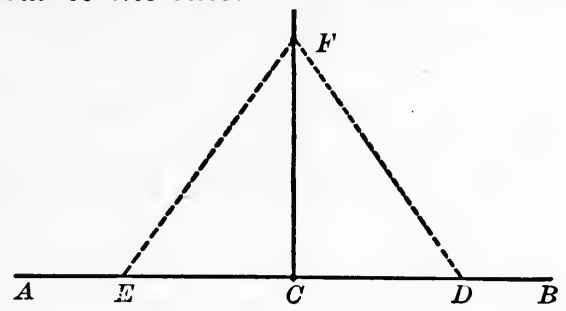

Let $A B$ be the given straight line and $C$ the given point in it.

It is required to draw from $C$ a straight line which is perpendicular to $A B$.

Construction. From $C$, on opposite sides, mark off any equal segments $C D$ and $C E$.

Upon the line-segment $E D$ as base, construct any isosceles triangle whose vertex is $F$. Draw the straight line $C F$.

Then $C F$ is perpendicular to $A B$.

Proof. Compare $\triangle \mathrm{s} F C E$ and $F C D$, making use of Proposition VIII. The triangles are identically equal.

Hence $\angle F C E$ equals $\angle F C D$ and each of them is a right angle.

Therefore $C F$ is the required line.

Compare the proof of this proposition with Ex. 9, p. 39.

For a proof that not more than one perpendicular can be drawn from any point, see Article 6

\section{EXERCISES}

1. Repeat the above construction, using the compasses and ruler, drawing only such arcs and lines as are necessary.

2. In the figure above, show that any point in the straight line $C F$, produced ever so far either way, is equidistant from $D$ and $E$, while a point of the plane, not on $C F$, is not equidistant from $D$ and $E$. 


\section{ON Locr}

72. If you should be given the problem to find a point in a plane equidistant from two given points in that plane, you would at once recognize that there are many points which will satisfy this condition. In fact, every point of a particular straight line fulfils the condition, while no point off that line does so. (Ex. 2, p. 49.)

This particular straight line is called the locus of the points fulfilling the given conditions.

The locus of points in a plane which are at a given distance from a given point is a circle of which the given point is the centre, and the given distance equal to a radius, since all points of the circle are at the given distance from the given point, and there is no other point of the plane which is at that distance from the given point.

73. Definitiox. If every point of a line or group of lines satisfies given conditions, while no other point satisfies those conditions, that line or group of lines is called the locus of the points satisfying the given conditions.

If any moving point continues to satisfy the given conditions, it is evident that it must follow the locus.

74. The properties of loci can be utilized for the solution of many problems in geometry, for example the problem, - to find a point which is equidistant from three given points $A, B$, and $C$.

The locus of points equidistant from $A$ and $B$ is the perpendicular bisector of the line-segment $A B$.

The locus of points equidistant from $B$ and $C$ is the perpendicular bisector of the line-segment $B C$.

If these two perpendicular bisectors $m$ and $n$ intersect at $O$, then $O$ is equidistant from $A, B$, and $C$, and is the only such point. 
For,

1. Every point of $m$ is equidistant from $A$ and $B$; Every point of $n$ is equidistant from $B$ and $C$.

$O$ is a point of both $m$ and $n$, and is therefore equidistant from $A, B$, and $C$.

2. No point outside of $m$ is equidistant from $A$ and $B$; No point outside of $n$ is equidistant from $B$ and $C$. Therefore, no point other than their intersection, can be equidistant from $A, B$, and $C$.

If $m$ and $n$ do not intersect, there is no point equidistant from $A, B$, and $C$.

\section{EXERCISES}

1. If lines are drawn to the extremities of a given line-segment from any point of its perpendicular bisector, these lines are not only equal but make equal angles, both with the given line-segment and with its perpendicular bisector.

2. Find a point in a given straight line such that its distance from two given points may be equal.

3. If from any point within a triangle line-segments are drawn to the extremities of one side, these are together less than the other two sides of the triangle.

Suggestion. Produce one of these line-segments backward to meet a side of the triangle, then apply Proposition XVI twice.

Notice what was proved concerning lines so drawn in Ex. 4, p. 45.

4. If $O$ be any point within a triangle $A B C$, show that the sum of $O A$, $O B$, and $O C$ is less than the sum of the sides of the triangle, but greater than half the sum of the sides.

5. The seginent of a straight line between any two points is shorter than any broken line connecting those points.

6. Find a point which is equidistant from two given points and at a given distance from a fixed point.

How many such points can there be ? Is it always possible to find a point which will satisfy the given conditions? 


\section{Proposition XVIII}

75. From a given point without a given straight line to draw a perpendicular to the line.

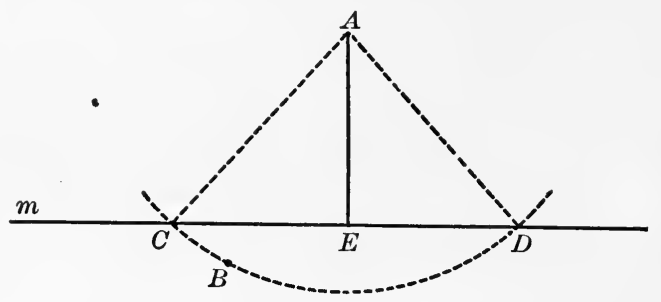

Let $m$ be the given straight line and $A$ the given point.

It is required to draw from $A$ a straight line perpendicular to $m$.

Construction. Choose a point $B$ on the opposite side of $m$ from $A$. With radius $A B$ describe a circle which must intersect $m$ in two points, say $C$ and $D$. (See Art. 25.)

Bisect the line-segment $C D$ at $E$. Join $A E$.

Then $A E$ is the required perpendicular.

Proof. Compare $\triangle \mathrm{s} A E C$ and $A E D$, using Proposition VIII. The triangles are identically equal, hence $\angle A E C$ equals $\angle A E D$, and therefore $A E$ is perpendicular to $m$.

The pupil should repeat this construction, drawing only such lines as are necessary to find the point $E$.

76. It was shown on p. 44 that from a point $A$ there could not be drawn two perpendiculars to the same straight line; that there can be one perpendicular, we have here shown by actually constructing it. Hence the theorem:

From a point outside a straight line, there can be drawn one perpendicular to that line, and only one.

The point $E$ in the diagram is called the foot of the perpendicular drawn from the point $A$ to the line $m$. 
77. By the distance from a point to a straight line, we mean the length of the line-segment between the given point and the foot of the perpendicular drawn from the point to the line. In other words, the distance from a point to a straight line is measured along the perpendicular from the point to the line.

\section{EXERCISES}

1. Of all the line-segments which can be drawn from a given point to a given straight line not passing through it, the perpendicular is the shortest.

2. From a given point line-segments are drawn to a given straight line, making equal angles with the perpendicular, on opposite sides ; prove that these line-segments are equal and intercept equal segments of the given line on opposite sides of the perpendicular ; and, conversely, if they intercept equal segments of the line, then they are equal and make equal angles with the perpendicular.

3. From a given point two line-segments are drawn to a given straight line, making unequal angles with the perpendicular; prove that the one which makes the greater angle with the perpendicular is the greater.

Suggestion. Let $P A$ and $P B$ be the lines, drawn say on the same side of the perpendicular $P M, P A$ making the lesser angle with it. Draw $P B^{\prime}$ on the opposite side of the perpendicular making $\angle B^{\prime} P M=\angle B P . M$. Then $\angle P A M$ is greater than $\angle P B M$ and therefore greater than $\angle P B^{\prime} M$.

4. Suppose $D$ is any point in one side $B C$ of a given triangle $A B C$. Find a straight line such that if the paper on which the triangle is drawn be folded along it, then $A$ will coincide with $D$.

5. A right triangle has one of its acute angles double the other. Prove that the hypotenuse is double the lesser side.

Suggestion. If $\triangle A B C$ is right-angled at $C$, and $\angle A$ double of $\angle B$, produce $A C$ to $D$ making $C D=A C$. Prove $A B D$ equilateral.

6. If $D$ is the mid-point of $B C$ the base of an isosceles triangle $A B C$, and $E$ any point in $A C$, prove that the difference between $B D$ and $D E$ is less than the difference between $A B$ and $A E$.

7. On one boundary of an angle whose vertex is $A$ points $B$ and $D$ are taken, and on the other boundary points $C$ and $E$, such that $A B$ is equal to $A C$, and $A D$ equal to $A E$; prove that $B E$ is equal to $C D$. 


\section{Proposition XIX}

78. If two right triangles have the hypotenuse and a side of one equal, respectively, to the hypotenuse and a side of the other, the triangles are identically equal.
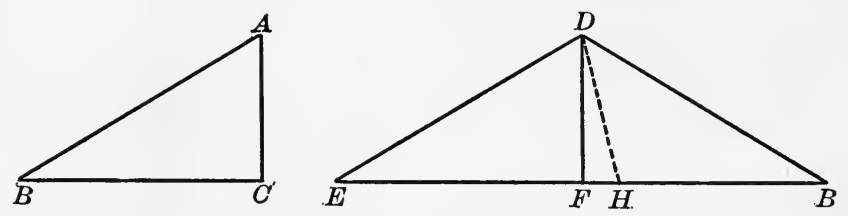

Let $A B C$ and $D E F$ be two triangles, right-angled at $C$ and $F$, respectively, having the hypotenuse $A B$ equal to the hypotenuse $D E$, and the side $A C$ equal to the side $D F$.

It is required to prove that the triangles $A B C$ and $D E F$ are identically equal.

Proof. Place $\triangle A B C$ beside $\triangle D E F$ so that the side $A C$ coincides with its equal side $D F$, the point $A$ with the point $D$, and the point $C$ with the point $F$, and so that the vertices $B$ and $E$ lie on opposite sides of $D F$.

Then $E F$ and $C B$ will lie in one straight line. Why?

Since by hypothesis $D E$ equals $A B$, in $\triangle D E B, D E$ equals $D B$, and therefore $\angle E$ equals $\angle B$. Give reference.

Again, if $B C$ is not equal to $E F$, then $F$ is not the mid-point of $E B$.

Suppose $H$ is the mid-point of $E B$, and join $D H$.

Then $D H$ must be perpendicular to $E B$ (Prop. VIII), and from $D$ there are drawn two perpendiculars to $E B$, which is impossible.

Therefore $H$ is not the mid-point of $E B$, and similarly it may be shown that no other point than $F$ is the mid-point.

Hence $E F$ and $F B$ are equal; that is, $E F$ and $B C$ are equal. Therefore $\triangle \mathrm{s} A B C$ and $D E F$ are identically equal. 


\section{Proposition XX}

79. If two triangles have two sides of the one equal, respectively, to two sides of the other, and the included angles unequal, the triangle which has the greater included angle has also the greater third side.
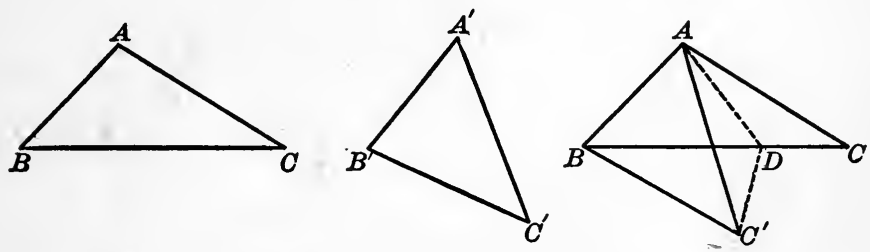

Let $A B C$ and $A^{\prime} B^{\prime} C^{\prime \prime}$ be the two given triangles in which the side $A B$ equals the side $A^{\prime} B^{\prime}$, the side $A C$ equals the side $A^{\prime} C^{\prime \prime}$, and the angle $B A C$ is greater than the angle $B^{\prime} A^{\prime} C^{\prime}$.

It is required to prove that the side $B C$ is greater than the side $B^{\prime} C^{\prime}$.

Proof. Place $\triangle A^{\prime} B^{\prime} C^{\prime \prime}$ upon $\triangle A B C$ so that the side $A^{\prime} B^{\prime}$ will coincide with the side $A B$, the point $A^{\prime}$ with the point $A$, and $B^{\prime}$ with $B$.

The side $A^{\prime} C^{\prime}$ will not coincide with the side $A C$ [why ?], but will fall within $\angle B A C$, while the point $C^{\prime}$ may fall outside $\triangle A B C$ as in the figure, or it may fall on the side $B C$, or within $\triangle A B C$, according to the lengths of the equal sides $A C$ and $A^{\prime} C^{\prime}$.

The pupil should draw figures for the other two cases. The proof is the same in all three cases.

Bisect $\angle C A C^{\prime}$ by the straight line $A D$, meeting $B C$ at $D$. Join $C^{\prime} D$.

$\triangle \mathrm{s} A C^{\prime \prime} D$ and $A C D$ are identically equal.

Therefore $C^{\prime \prime} D$ equals $C D$.

(Prop. IV.) 
Hence $B D$ and $D C^{\prime}$ are together equal to $B C$.

But $B D$ and $D C^{\prime \prime}$ are together greater than $B C^{\prime}$. Why?

'Therefore $B C^{\prime}$ is greater than $B C^{\prime \prime}$. 'That is, $B C$ is greater than $B^{\prime} C^{\prime}$.

\section{Proposition XXI}

80. If two triangles have two sides of the one equal, respectively, to two sides of the other, and the third sides unequal, the triangle which has the greater third side has also the greater included angle.
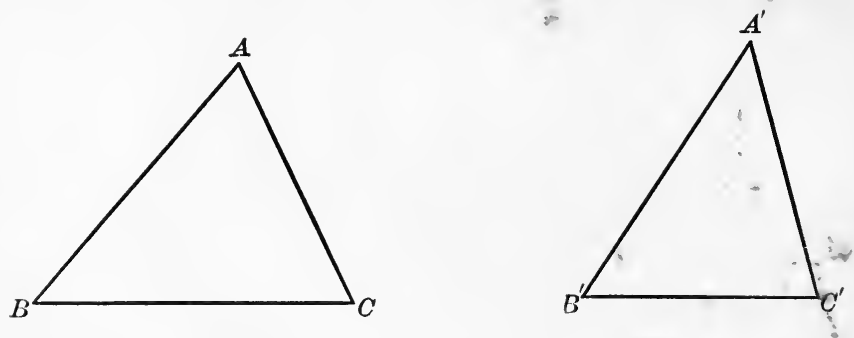

Let $A B C$ and $A^{\prime} B^{\prime} C^{\prime}$ be the given triangles having the side $A B$ equal to the side $A^{\prime} B^{\prime}, A C$ equal to $A^{\prime} C^{\prime}$, but the third side $B C$ greater than the third side $B^{\prime} C^{\prime}$.

It is required to prove that the included angle $B A C$ is greater than the included angle $B^{\prime} A^{\prime} C^{\prime}$.

Proof. If $\angle B A C$ is not greater than $\angle B^{\prime} A^{\prime} C^{\prime}$, it must be equal to, or less than $\angle B^{\prime} A^{\prime} C^{\prime \prime}$.

First, suppose $\angle B A C$ is equal to $\angle B^{\prime} A^{\prime} C^{\prime}$.

Then the side $B C$ must equal the side $B^{\prime} C^{\prime}$.

(Prop. IV.)

But this is contrary to the hypothesis.

Next, suppose $\angle B A C$ is less than $\angle B^{\prime} A^{\prime} C^{\prime}$.

Then the side $B C$ must be less than $B^{\prime} C^{\prime}$.

(Prop. XX.)

But this is also contrary to the hypothesis.

Therefore $\angle B A C$ must be greater than $\angle B^{\prime} A^{\prime} C^{\prime}$.

What kind of proof do you call this? 


\section{Proposition XXII}

81. If two triangles have two sides of the one equal, respectively, to two sides of the otleer, and the angles opposite one pair of equal sides equal, then the angles opposite the other pair of equal sides are either equal or supplementary, and if equal, then the triangles are identically equal.
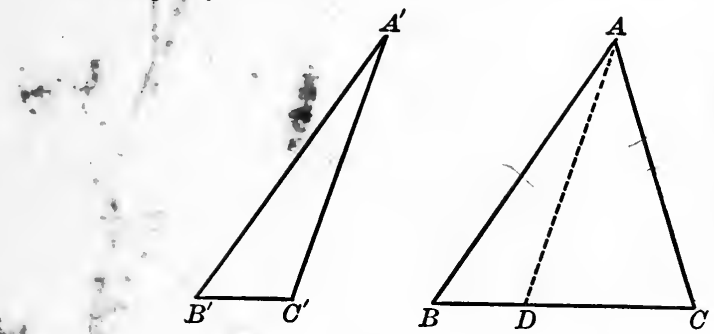

Let $\ldots 2$ and $A^{\prime} B^{\prime} C^{\prime}$ be two triangles having the side $A B$. equal to the side $A^{\prime} B^{\prime}, A C$ equal to $A^{\prime} C^{\prime}$, and the angle at $B$ eq. $z^{\text {tho }}$ ingle at $B^{\prime}$, these being opposite equal sides.

It is required to prove that the angles $A C B$ and $A^{\prime} C^{\prime \prime} B^{\prime}$ are either equal or supplementary, and if equal, then the triangles are identically equal.

Proof. Place $\triangle A^{\prime} B^{\prime} C^{\prime}$ upon $\triangle A B C$ so that the side $A^{\prime} B^{\prime}$ will coincide with its equal side $A B$, the point $A^{\prime}$ with the point $A$, and the point $B^{\prime}$ with the point $B$.

Then since $\angle B^{\prime}$ equals $\angle B$, the side $B^{\prime} C^{\prime \prime}$ will fall on the side $B C$, and $C^{\prime}$ will either coincide with $C$ or with some other point of the straight line $B C$.

If $C^{n}$ coincides with $C$, the two triangles coincide throughout and are identically equal.

If $C^{\prime}$ falls at some other point, as $D, \angle A D C$ equals $\angle A C D$ [why?], and $\angle A D B$ is supplementary to $\angle A D C$, or $\angle A C B$.

That is, $\angle A^{\prime} C^{\prime} B^{\prime}$ is either equal to $\angle A C B$ or supplementary to $\angle A C B$, and if equal the triangles are identically equal. 
82. Corollary. If the two angles given equal are right angles or obtuse angles, the two triangles must be identically equal.

Proposition XIX thus becomes a special case of Proposition XXII.

Cases in which Two Triangles are Identically Equal

83. It may be well at this point to collect in tabulated form the theorems which have already been proved relating to the identical equality of two triangles.

Two triangles are identically equal when the following parts in each are respectively equal:

1. Two sides and the included angle.

(Prop. IV.)

2. Two angles and the adjacent side.

(Prop. V.)

3. The three sides.

(Prop. VIII.)

4. A right angle, the hypotenuse, and a side.

(Prop. XIX.)

5. Two sides and an angle opposite one of them.

(Prop. XXII.)

It should be noticed that in each of the five cases there are three parts of the one triangle equal, respectively, to three parts of the other.

It must not, however, be concluded that if any three parts of one triangle are respectively equal to the three corresponding parts of another, the triangles are identically equal. For example, it is not necessarily true that if the three angles of one triangle are respectively equal to the three angles of another, the triangles are identically equal.

In every case it is necessary that at least one of the three given parts should be a side.

If the three given parts are two sides and an angle opposite one of them, as in Case 5, there is an additional condition necessary, namely, that the angles opposite one pair of equal sides being equal, the angles opposite the other pair shall also be equal, not supplementary. 


\section{Proposition XXIII}

84. At a given point on a given straight line to construct an angle equal to a given angle.
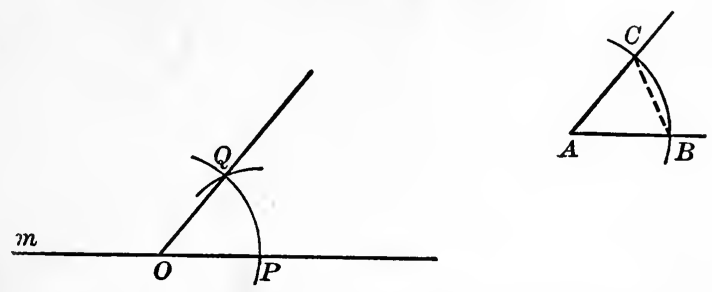

Let $A$ be the given angle, $m$ the given straight line, and $O$ the given point on it.

It is required to construct an angle equal to $A$, having its vertex at $O$, and having the line $m$ for one boundary.

Construction. With the point $A$ as centre describe a circle of any radius cutting the boundaries of the given angle at $B$ and $C$.

With $O$ as centre and the same radius describe a circle cutting the line $m$ at the point $P$.

With centre $P$ and radius equal to the line-segment $B C$ describe a circle cutting the former circle at $Q$. Draw $O Q$.

The angle $P O Q$ is the required angle.

Proof. Join $B C$; also $P Q$. Prove $\triangle P O Q$ identically equal to $\triangle B A C$ (Prop. VIII), and hence $\angle O$ equal to $\angle A$.

How many such angles can be constructed at $O$ ?

\section{EXERCISES}

1. At a given point in a given straight line construct an angle equal to the supplement of a given angle.

2. If the straight line bisecting the vertical angle of a triangle also bisects the base, the triangle is isosceles. (Apply Proposition XXII.)

3. Prove Proposition VIII indirectly by assuming the included angles between two pairs of corresponding sides unequal. 


\section{MISCELLANEOUS EXERCISES}

1. If one angle of a triangle is equal to the sum of the other two, the triangle can be divided into two isosceles triangles.

2. If the angle $C^{\prime}$ of a triangle $A B C$ is equal to the sum of the angles $A$ and $B$, the side $A B$ is equal to twice the straight line joining $C$ to the mid-point of $A B$.

3. $A$ is a given point, and $B$ a given point in a given straight line. It is required to draw from $A$ to the given straight line, a line-segment $A P$ such that the sum of $A B$ and $B P$ may be of given length.

4. If $B E$ and $C F$ are drawn from the extremities of the base of an isosceles triangle $A B C$ meeting the equal sides at $E$ and $F$, and making equal angles with the bases then $\triangle B C F$ and $C B E$ are identically equal.

5. The equal sides $A B$ and $A C$ of an isosceles triangle are produced to $E$ and $F$, respectively, so that $A E$ equals $A F ; B F$ and $E C$ are joined ; show (1) that $B F$ equals $E C$, (2) that $\angle C B F$ equals $\angle B C E$, (3) $\angle A B C$ equals $\angle A C B$, making use only of P'roposition IV.

Note. This is Euclid's order of proving that the angles at the base of an isosceles triangle are equal.

6. On the same base and on the same side of it there are given two triangles, $A C B$ and $A D B$, such that the sides $A C$ and $A D$ are equal. Show that the sides $B C$ and $B D$ cannot be equal.

Note. This is Euclid's Proposition VII of Book I.

7. In a triangle $A B C$ in which $A B$ is greater than $A C$, if the bisector of the angle at $A$ meets $B C$ at $D$, show that $B D$ is greater than $C D$.

Scggestrox. In $A B$ make $A E$ equal to $A C^{\circ}$, join $D E . \triangle \mathrm{s} A D E$ and $A D C$ are identically equal. Then apply Proposition XII and P'roposition XV.

8. Show that the line drawn from the vertex of a triangle to any point in the base (between the vertices) is less than the greater of the two sides, or than either of them if they are equal.

9. If $D$ is the mid-point of the side $B C$ of a triangle $A B C$, in which $A C$ is greater than $A B$, the angle $A D C$ is obtuse.

10. If in the sides $A B, A C$ of a triangle $A B C$, in which $A C$ is greater than $A B$, points $D$ and $E$ are taken such that $B D$ and $C E$ are equal, then $C D$ is greater than $B E$. 


\section{Section II}

\section{PARALLEL LINES}

85. If we have given a point $O$ and a straight line $a$, not passing through it, and draw through $O$ any straight line $p$,

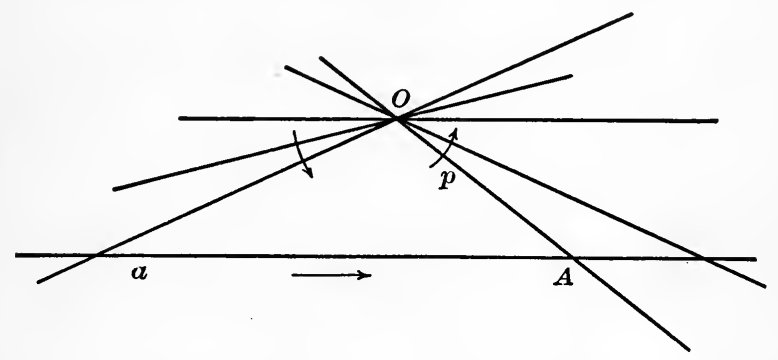

unlimited on either side of $O$, it will ordinarily intersect $a$ in some point $A$. If now the line $p$ is rotated about $O$ as indicated by the arrow-head, $p$ and $a$ will continue to intersect and the point of intersection $A$ will move off to the right. As the rotation proceeds, the lines will by and by intersect far out to the left, but the point of intersection will still move from the left to the right.

The question arises, Do these lines always intersect? and we are accustomed to say, No, there is one position of $p$ for which they do not intersect. When $p$ is in that position it is said to be parallel to $a$.

Definition. Parallel straight lines are straight lines lying in the same plane, and such that if they are drawn ever so far either way they will not intersect.

Straight lines in the same plane which are not parallel must intersect, if produced far enough.

86. Postulate 5. Through any point one and only one straight line can be drawn parallel to a given straight line. 
87. If two straight lines, parallel or not, are intersected by a third line, called a transversal, four angles are made at each intersection. These may be designated by writing a letter in each angle, and calling the angle by the name of the letter.

At each intersection there are two pairs of vertical angles and four pairs of adjacent angles.

Other pairs of angles have

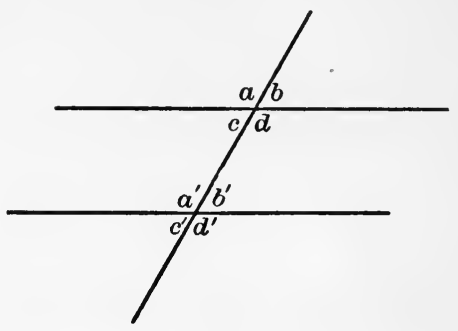
also received special names. Thus the angles $a$ and $a^{\prime}, b$ and $b^{\prime}, c$ and $c^{\prime}, d$ and $d^{\prime}$ are called corresponding angles.

The angles $a$ and $d^{\prime}, b$ and $c^{\prime}, c$ and $b^{\prime}, d$ and $a^{\prime}$, are called alternate angles.

The angles $a, b, c^{\prime}, d^{\prime}$, are called exterior angles, and the angles $c, d, a^{\prime}, b^{\prime}$, are called interior angles.

\section{Propositión XXIV}

88. If two straight lines lying in one plane are such that a transversal makes the interior alternate angles equal these two lines are parallel.

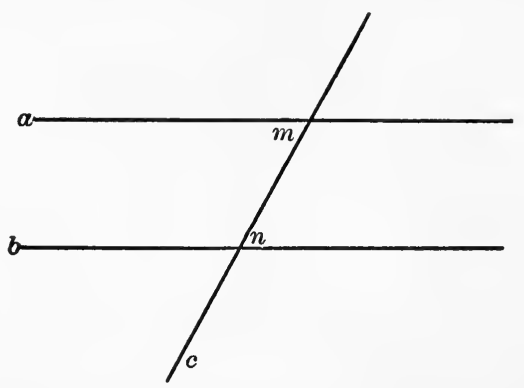

Let the straight lines $a$ and $b$ lie in one plane and be such that the transversal $c$ makes the alternate angles $m$ and $n$ equal. 
It is required to prove that $a$ and $b$ are parallel.

Proof. If $a$ and $b$ are not parallel they will intersect if produced far enough either one way or the other.

If they intersect, they and the transversal will form a triangle in which one of the two angles $m$ and $n$ will be an exterior angle formed by producing a side, and the other, an interior non-adjacent angle, in which case they could not be equal.

(Prop. XII.)

Hence $a$ and $b$ cannot intersect, and therefore must be parallel.

Exercise. Show that if any one pair of alternate angles are equal, all pairs are equal.

89. CoRollary I. If two straight lines lying in one plane are such that a transversal makes a pair of corresponding angles equal, these two lines are parallel.

Prove by showing that if a pair of corresponding angles are equal a pair of alternate angles must also be equal.

90. Corollary II. If two straight lines lying in one plane are such that a transversal makes a pair of interior angles, or a pair of exterior angles, on the same side of it supplementary, these two lines are parallel.

91. Corollary III. Straight lines lying in one plane and perpendicular to the same straight line are parallel.

92. Corollary IV. Two straight lines parallel to the same straight line are parallel to each other.

For, if they are not parallel they must intersect when produced; then there would be through their point of intersection two straight lines parallel to the same line, contrary to Postulate 5 . 


\section{Proposition XXV}

93. If two parallel straight lines are cut by any transversal, the interior alternate angles are equal.

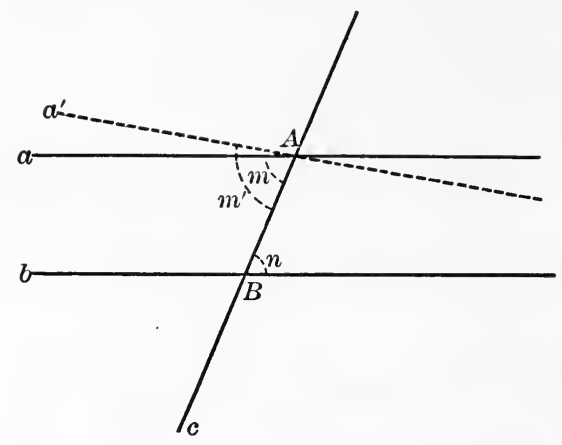

Let $a$ and $b$ be two parallel lines and $c$ any transversal intersecting $a$ at $A$ and $b$ at $B$.

It is required to prove that the interior alternate angles $m$ and $n$ are equal.

Proof. If $\angle m$ is not equal to $\angle n$, through $A$ draw the straight line $a^{\prime}$ making with the transversal $c$ an angle $m^{\prime}$ which is equal to $\angle n$.

Then the straight lines $a^{\prime}$ and $b$ must be parallel. (Prop. XXIV.)

But, by hypothesis, $a$ and $b$ are parallel.

Therefore, since through $A$ but one straight line can be drawn parallel to $b, a^{\prime}$ must coincide with $a$, and $\angle m^{\prime}$ must coincide with $\angle m$.

But by construction $\angle m^{\prime}=\angle n$.

Therefore $\angle m=\angle n$.

94. Corollary I. If two parallel lines are cut by a transversal, any two alternate angles and any two corresponding angles are equal. 
95. CoRollary II. If two parallel lines are cut by any transversal, the interior angles, and also the exterior angles, on the same side of the transversal, are supplementary.

96. CoRollakx III. If any pair of alternate angles formed by two straight lines and a transversal are unequal, or if any pair of corresponding angles are unequal, or if the interior angles on one side are not supplementary, the two lines are not parallel and therefore will meet if produced.

For if the lines are parallel, we have just proved that alternate angles must be equal, corresponding angles must be equal, and interior angles on one side must be supplementary, contrary to the assumption.

97. Corollary IV. If a straight line is perpendicular to one of two parallel lines, it is perpendicular also to the other.

98. Corollary V. If two straight lines intersect, lines perpendicular to them in the same plane will also intersect.

Let $a$ and $b$ be two intersecting straight lines and let $c$ be perpendicular to $a$, and $d$ perpendicular to $b$. If $c$ and $d$ do not intersect, they must be parallel, and then since $a$ is perpendicular to $c$ it must also be perpendicular to $d$ (Cor. IV). Hence $a$ and $b$ are both perpendicular to $d$ and must themselves be parallel (Art. 91), contrary to hypothesis.

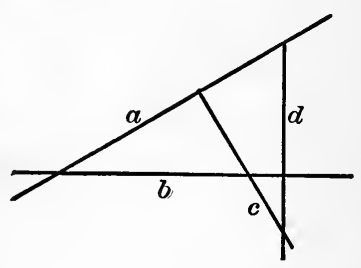

\section{EXERCISES}

1. Any straight line drawn parallel to the base of an isosceles triangle forms with the sides another isosceles triangle.

2. Is it always true that if two angles are equal and one boundary of the one parallel to one boundary of the other, then their other boundaries are also parallel?

Illustrate your answer by diagrams.

3. Prove that two parallel lines are everywhere equidistant.

4. If any two straight lines intersect, lines parallel to them must also intersect. 


\section{Proposition XXVI}

99. Through a given point to draw the straight line parallel to a given straight line.

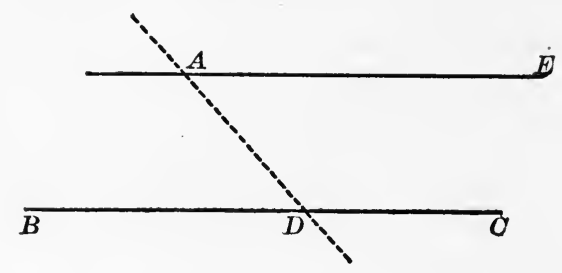

Let $A$ be the given point and $B C$ the given straight line.

It is required to draw through $A$ the straight line parallel to $B C$.

Construction. Through $A$ draw any straight line intersecting $B C$ at a point $D$.

At $A$, in the straight line $A D$, construct $\angle D A E$ equal to $\angle A D B$.

(Prop. XXIII.)

Then the straight line $A E$ is parallel to $B C$. (Prop. XXIV.)

\section{EXERCISES}

1. Through a given point draw a straight line making with a given straight line an angle equal to a given angle. How many such lines can be drawn?

2. Two line-segments $A B$ and $C D$ bisect each other at $O$. Prove that the lines $A C$ and $B D$ are parallel. How about $A D$ and $B C$ ?

3. If through the vertex of an isosceles triangle a straight line is drawn parallel to the base, it will bisect the exterior vertical angle.

4. Through a given point draw a straight line which will make equal angles with two given intersecting straight lines.

Suggestrox. Draw the perpendicular to the bisector of the angle between the lines. 


\section{Proposition XXVII}

100. If one side of a triangle is produced, the exterior angle so formed is equal to the sum of the two interior non-adjacent angles.

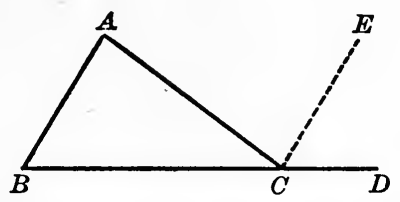

Let $A B C$ be any triangle having the side $B C$ produced to $D$.

It is required to prove that the exterior angle $A C D$ is equal to the sum of the two interior non-adjacent angles, viz. the angles $C A B$ and $C B A$.

Proof. From $C$ draw the straight line $C E$ parallel to $B A$.

(Prop. XXVI.)

Then $\angle E C D=\angle C B A$ and $\angle A C E=\angle C A B$. Why?

Therefore the sum of $\angle \mathrm{s} A C E$ and $E C D$ equals the sum of $\angle \mathrm{s} C A B$ and $C B A$

That is, $\angle A C D$ equals the sum of $\angle \mathrm{s} C A B$ and $C B A$.

101. Corollary I. The sum of the three angles of a triangle is equal to two right angles.

102. Corollary II. The two acute angles of any right triangle are complementary; i.e. their sum is a right angle.

Definition. One angle is said to be the complement of another when the sum of the two is a right angle.

103. Corollary III. Each angle of a triangle is the supplement of the sum of the other two. 
104. Corollary IV. If two triangles have two angles of the one equal respectively to two angles of the other, their third angles are also equal; or, if the sum of two angles in the one is equal to the sum of two angles in the other, their third angles are equal.

105. Corollary V. If the boundaries of one angle are respecticely parallel or perpendicular to the boundaries of another, these two angles are either equal or supplementary.

Make diagrams representing all the cases.

106. Corollary VI. If two triangles have two angles of the one equal respectively to two angles of the other, and the sides opposite one pair of equal angles also equal, the triangles are identically equal.

For then the third angles are also equal, and the thenrem falls under Proposition V. This theorem may be easily proved by superposition, making use of Proposition XII.

\section{EXERCISES}

1. If an isosceles triangle is right-angled, each of the angles, at the base is half a right angle.

2. If two isosceles triangles have their vertical angles equal, they are mutually equiangular; i.e. each angle of the one is equal to an angle of the other.

3. If one angle of a triangle is equal to the sum of the other two, it must be a right angle.

4. Divide a right triangle into two isosceles triangles, and hence show that the midpoint of the hypotenuse is equidistant from the three vertices.

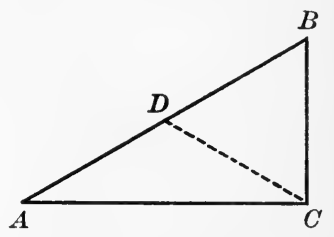

Suggestion. Construct $\angle A C D=\angle A$, and show that $\angle B C D=\angle B$. Hence $D A=D C=D B$.

5. Each angle of an equilateral triangle is two-thirds of a right angle. 


\section{Proposition XXVIII}

107. Every point on the bisector of an angle is equidistant from the boundaries of the angle; and every point within the angle which is equidistant from the bounduries is on the bisector; that is, the bisector of an angle is the locus of points within the angle which are equidistant from its boundaries.

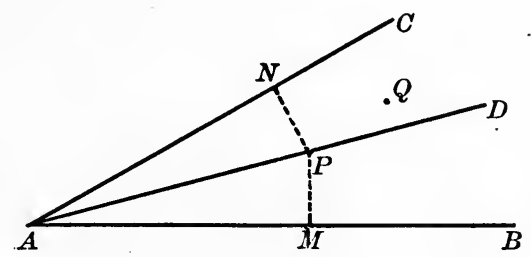

First, let $A D$ be the bisector of the angle $B A C$ and let $P$ be any point on $A D$.

It is required to prove that $P$ is equidistant from $A B$ and $A C$.

Proof. From $P$ draw the lines $P M$ and $P N$ perpendicular, respectively, to $A B$ and $A C$.

Then $\triangle \mathrm{s} P A M$ and $P A N$ are identically equal. Why? (Apply Prop. V.)

Therefore $P M$ equals $P N$; that is, $P$ is equidistant from $A B$ and $A C$.

Next, let $Q$ be any point equidistant from $A B$ and $A C$ within the angle $B A C$.

It is required to prove that $Q$ lies on the bisector of the angle $B A C$.

Proof. From $Q$ draw $Q S$ and $Q T$ perpendicular to $A B$ and $A C$, respectively, and join $Q A$.

By hypothesis, $Q S$ and $Q T$ are equal. 
Then $\triangle \mathrm{s} Q S A$ and $Q T A$ are identically equal. Why? (Apply Prop. XIX.)

Therefore $\angle Q A S$ equals $\angle Q A T$; that is, $Q$ lies on the bisector of the angle $B A C$.

Since the bisector of the angle $B A C$ contains all points within the angle which are equidistant from the boundaries, and no point which is not equidistant from the boundaries, it is the locus of such points.

108. Corollary. The locus of points equidistant from two intersecting straight lines consists of the two lines which bisect the angles formed by the given lines.

\section{EXERCISES}

1. Prove that the locus of points equidistant from two intersecting straight lines consists of two straight lines at right angles.

2. If the straight line drawn from one vertex of a triangle to the midpoint of the opposite side is equal to half of this side, prove that the triangle has one right angle.

3. If, in a right triangle, a perpendicular is drawn from the vertex of the right angle to the opposite side, the two triangles so formed are equiangular with each other and with the whole triangle.

4. A straight line drawn perpendicular to the base $B C$ of an isosceles triangle $A B C$ meets the side $A B$ at $E$ and the side $C A$ produced at $F$. Prove that the triangle $E A F$ is isosceles.

5. If a point is equidistant from two parallel straight lines, any linesegment drawn through it and terminated by the parallel lines is bisected at the point.

6. If a point is equidistant from two parallel straight lines, any two straight lines drawn through it intercept equal segments of the parallel lines.

7. Construct a triangle having given two angles and the length of the perpendicular from the third vertex to the opposite side.

8. If two exterior angles of a triangle be bisected, and from the point of intersection of the bisecting lines a straight line is drawn to the third vertex, it bisects the third angle. (Apply Proposition XXVIII.) 


\section{Section III}

\section{CLOSED RECTILINEAR FIGURES OF MORE THAN} THREE SIDES

109. On page 13 a closed figure was defined as one which can be traversed by starting at any point of it, and moving continuously along the lines of the figure in order, returning to the same point without passing twice over any portion of the figure.

A closed rectilinear figure is one which is made up wholly of line-segments and the points in which they intersect, two and two, in order.
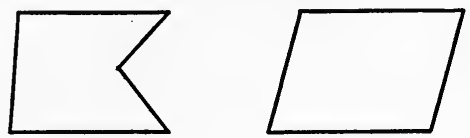

The line-segments are called the sides of the figure, and the points in which the sides intersect are called the vertices of the figure.

Two sides which intersect in a vertex are called adjacent sides.

The angles formed by pairs of adjacent sides are called the angles of the figure.

A straight line joining any two vertices not on the same side is a diagonal.

In the diagram, the points $A, B, C, D, E$ are vertices, the line-segments $A B, B C, C D$, etc., are sides, and $A C, A D, C E$, etc., are diagonals.

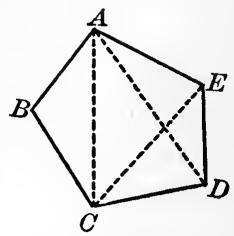

If the number of sides of any closed rectilinear figure is $n$, the number of diagonals which can pass through any one vertex is $n-3$, and since each diagonal passes through two vertices, the total number of diagonals is $\frac{1}{2} n(n-3)$. 
110. A convex rectilinear figure is one which lies wholly on one side or the other of each of its sides.

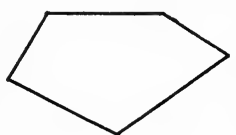

FIG. 1

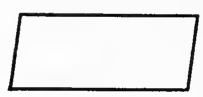

FIG. 2

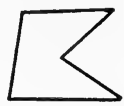

FIG. 3

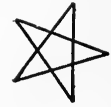

FIG. 4

Figures 1 and 2 above are convex rectilinear figures, while figures 3 and 4 are closed but not convex.

Could a triangle be anything else than convex?

111. We shall usually denote a closed rectilinear figure by placing capital letters at its vertices and naming them in order. 'Thus, the figure at the right is the figure $A B C D E F$, or $C B A F E D$, etc. When no misunderstanding is likely to arise, the figure may be named by mentioning two non-consecutive vertices.

Sometimes it will be convenient to letter the sides of the figure $a, b, c, d, e, f$, and then the figure can be named by mentioning the sides in order.

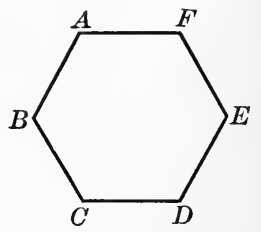

112. It will be observed that in any closed rectilinear figure there are just as many vertices as sides. The vertices and sides together make up the elements of the figure, so that the number of elements is always even, and equal to twice the number of sides of the polygon.

113. Two elements are said to be opposite when just as many elements lie between them the one way round as the other way round the figure.

If the number of sides in the figure is even, a side is always opposite a side, and a vertex opposite a vertex. In the figure above, the side $C D$ is opposite the side $A F$, the vertex $B$ opposite the vertex $E$, etc. the number of elements between the pairs of opposite elements being in each case five.

If the number of sides in the figure is odd, a side is always opposite a vertex.

We have already assumed that the pupil will know the opposite elements in a triangle; it is not, however, so easy to detect opposite elements when the figure has more than three sides. 
114. A closed rectilinear figure having three sides is a triangle, one of four sides is a quadrilateral.

A general name applied to any closed rectilinear figure, without regard to the number of sides, is a polygon.

115. A convex polygon is a closed rectilinear figure which lies wholly on one side or the other of each of its sides.

\section{Proposition XXIX}

116. The sum of the interior angles of any convex polygon, together with four right angles, makes up twice as many right angles as the polygon has sides.

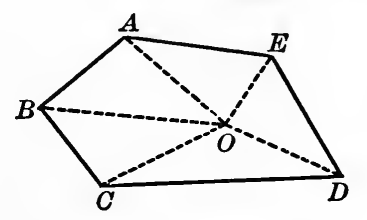

Let $A B C D E$ be any convex polygon.

It is required to prove that the sum of the interior angles $A, B, C, D, E$, together with four right angles, makes up twice as many right angles as the figure has sides.

Proof. Choose any point $O$ within the polygon, and join $O$ to each vertex of the polygon.

This divides the polygon into as many triangles as the polygon has sides.

The three angles of each triangle are together equal to two right angles.

(Prop. XXVII, Cor. I.)

Hence all the angles of all the triangles are together equal to two right angles for each side, or to twice as many right angles as the polygon has sides.

But these angles include the interior angles of the polygon, together with four right angles about the point $O$.

Therefore ... 
117. Corollary I. If the sides of a convex polygon are produced in order, the exterior angles so formed are together equal to four right angles.

Suggestion. Each exterior angle and its adjacent interior angle make two right angles.

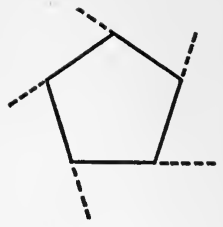

118. CoRollary II. If $S$ represents the sum of the interior angles of a convex polygon, and $n$ the number of sides, then $S+$ four right angles $=2 n$ right angles, or $S=(n-2)$ times two right angles.

\section{Definitions}

119. A parallelogram is a quadrilateral whose opposite sides are parallel.

If only two opposite sides of a quadrilateral are parallel, the figure is called a trapezoid.

If no two sides of a quadrilateral are parallel, the figure is called a trapezium.

120. A parallelogram having two adjacent sides equal is called a rhombus.

121. A parallelogram having one right angle is called a rectangle.

122. A square is a parallelogram having one right angle and two adjacent sides equal.

\section{EXERCISES}

1. The sum of the interior angles of any quadrilateral is equal to four right angles.

To how many right angles is the sum of the interior angles of a convex polygon of five sides equal ? of six sides ? of eight sides?

2. If two angles of a quadrilateral are right angles, the other two are supplementary.

3. If any one angle of an isoceles triangle equals two thirds of a right angle, the triangle is equilateral. 


\section{Proposition XXX}

123. The opposite sides and the opposite angles of a parallelogram are equal.

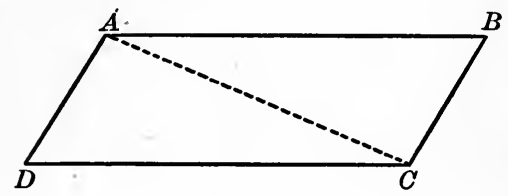

Let $A B C D$ be a parallelogram.

It is required to prove that $A B$ is equal to $D C$ and $A D$ equal to $B C$; also that the angle $A$ is equal to the angle $C$, and the angle $B$ equal to the angle $D$.

Proof. Join a pair of opposite vertices $A$ and $C$.

Because $A B$ is parallel to $D C$, by definition,

$$
\angle B A C=\angle D C A \text {. }
$$

(Prop. XXV.)

And because $A D$ is parallel to $B C$,

$$
\angle B C A=\angle D A C \text {. }
$$

Therefore $\triangle \mathrm{s} A B C$ and $C D A$ are identically equal. (Prop. V.) Conclusion . . .

124. Corollary. Either diagonal of a parallelogram divides the figure into two superposable triangles.

125. In writing out the proofs of propositions and in the exercises, the pupil is advised to make use of certain well established symbols in order to abbreviate his work. For instance, instead of the expression 'is parallel to,' or the word 'parallel,' he may use the symbol \|; and instead of 'is perpendicular to,' or ' perpendicular,' he may use the symbol $\perp$. Thus ' $A B$ is parallel to $C D$ ' would be written ' $A B \| C D$,' and ' $A B$ is perpendicular to $C D$ ' would be written ' $A B \perp C D$ '. The pupil is also advised to make free use of algebraic symbols in all written work. 


\section{Proposition XXXI}

126. The diagonals of a parallelogram bisect each other.

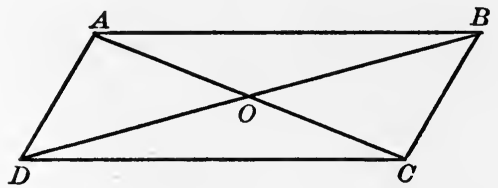

Let $A B C D$ be a parallelogram whose diagonals intersect at $O$.

It is required to prove that $O$ is the mid-point of both $A C$ and $B D$.

Proof. Compare the two triangles $A O B$ and $C O D$, making use of Propositions XXV, XXX, and V.

\section{Proposition XXXII}

127. If two sides of a quadrilateral are parallel and equal, the figure is a parallelogram.

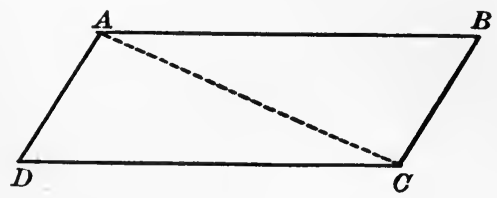

Let $A B C D$ be a quadrilateral having the sides $A B$ and $D C$ parallel and equal.

It is required to prove that $A D$ and $B C$ are parallel, and hence that the figure is a parallelogram.

Proof. Draw a diagonal $A C$. Then $\triangle \mathrm{s} B A C$ and $D C A$ are identically equal by Propositions XXV and IV.

Therefore

$$
\angle B C A=\angle D A C \text {, }
$$

and the sides $A D$ and $B C$ are parallel.

(Prop. XXIV.) 


\section{Proposition XXXIII}

128. If both pairs of opposite sides of a quadrilateral are equal, the figure is a parallelogram.

Given a quadrilateral whose opposite sides are equal.

It is required to prove that the opposite sides are parallel, and hence that the figure is a parallelogram.

Proof. Draw a diagonal and compare the triangles so formed. [The pupil should make the necessary diagram for himself.]

\section{Proposition XXXIV}

129. If two parallelograms have two adjacent sides and the included angle of the one equal, respectively, to two adjacent sides and the included angle of the other, the parallelograms are identically equal.

Prove by superposition, placing the one parallelogram on the other so that parts given equal will coincide, and showing that the remaining parts must also coincide.

\section{EXERCISES}

1. If the diagonals of a quadrilateral bisect each other, the quadrilateral is a parallelogram.

2. In a parallelogram the perpendiculars drawn from one pair of opposite vertices, to the diagonal through the other pair, are equal.

3. If the diagonals of a parallelogram are equal, the parallelogram is rectangular.

4. If $A B C D$ is a parallelogram, and $X, Y$, respectively, the mid-points of the sides $A D, B C$; show that the figure $A Y C X$ is a parallelogram.

5. If both pairs of opposite angles of a quadrilateral are equal, the figure is a parallelogram.

6. If one angle of a parallelogram is a right angle, all the angles are right angles.

7. If two sides of a quadrilateral are parallel, and the other two equal but not parallel, the diagonals are equal.

Such a figure is called an isosceles trapezoid. 


\section{Section IV \\ MISCELLANEOUS THEOREMS \\ Proposition XXXV}

130. The line-segment joining the mid-points of two sides of a triangle is parallel to the third side and equal to half of it.

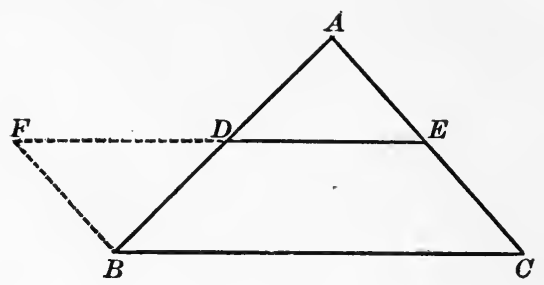

Let $A B C$ be any triangle, $D$ and $E$ the mid-points of the two sides $A B$ and $A C$.

It is required to prove that the line-segment $D E$ is parallel to the third side $B C$, and equal to half of it.

Proof. From $B$ draw $B F$ parallel to $C A$, meeting the straight line $E D$ in $F$.

$\triangle B D F$ is identically equal to $\triangle A D E$. Prove.

Therefore $D F=D E$, and $B F=A E$.

But $A E=C E$ by hypothesis.

Therefore $B F=C E$, and is parallel to $C E$ by construction. Therefore $B F E C$ is a parallelogram (Prop. XXXII), and $F E$, i.e. $D E$, is parallel to $B C$.

It is left for the pupil to prove that $D E$ equals half of $B C$.

131. Conollary I. If the mid-points of the sides of any quadrilateral be joined in order, the figure so formed is a paral- 
lelogram, and the sum of the sides of this parallelogram equals the sum of the diagonals of the quadrilateral.

Suggestion. Draw the diagonals of the quadrilateral and apply Proposition XXXV.

132. Definition. The sum of the sides of any polygon is called the perimeter of the polygon.

133. Corollary II. If from the mid-point of one side of a triangle there is drawn a straight line parallel to a second side, this line passes through the mid-point of the third side.

Else through one point there could be drawn two parallels to the same line.

134. Definition. A straight line drawn from any vertex of a triangle to the mid-point of the opposite side is called a median of the triangle. Every triangle has three medians.

135. Definition. By concurrent lines we mean lines which pass through the same point.

\section{EXERCISES}

1. The mid-points of a pair of opposite sides of a quadrilateral and the mid-points of the diagonals are the vertices of a parallelogram.

2. In a trapezoid the mid-points of the non-parallel sides and the midpoints of the diagonals lie upon the same straight line parallel to the other two sides. The distance between the mid-points of the non-parallel sides is equal to half the sum of the parallel sides; and the distance between the mid-points of the diagonals is equal to half the difference between the parallel sides.

3. Find on one side of a triangle the point from which straight lines drawn parallel to the other two sides, and terminated by these sides, are equal.

Suggestion. Bisect the opposite angle.

4. If $A B C D$ and $A E F G$ are two squares so situated that the angles $A$ coincide, then $A, C$, and $F$ are in the same straight line.

5. If two parallelograms have a diagonal and two vertices in common, show that the other four vertices are the vertices of another parallelogram. 


\section{Proposition XXXVI}

136. The medians of a triangle are concurrent.

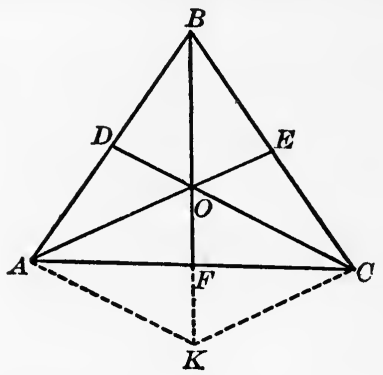

Let $A B C$ be any triangle, $A E$ and $C D$, two of its medians meeting at $O$. Join $B O$ and produce to meet $A C$ at $F$.

It is required to prove that $F$ is the mid-point of $A C$, and hence that $B F$ is the third median of the triangle.

Proof. Through $A$ draw $A K$ parallel to $D C$, meeting $B F$ at $K$. Join $C K$.

Then in $\triangle B A K$, since $B D=D A, B O=O K$.

(Prop. XXXV., Cor. II.)

In $\triangle B C K$, since $B E=E C$, and $B O=O K$,

$E O$ is parallel to $C K$; that is, $O A$ is parallel to $C K$.

Therefore $A O C K$ is a parallelogram, and $A F$ equals $F C$. Therefore $B F$, passing through $O$, is a median of the triangle.

(Prop. XXXI.)

137. Definition. The point $O$, in which the medians intersect, is called the centroid of the triangle.

138. Corollary. The centroid of a triangle is a trisection point of each median of the triangle.

For, since $O K=O B$, and $O F=\frac{1}{2} O K$, therefore, $O F=\frac{1}{2} O B$, or $\frac{1}{3} B F$.

Similarly $O$ may be shown to be a point of trisection of the other medians. 


\section{Proposition XXXVII}

139. The bisectors of the angles of a triangle are concurrent.

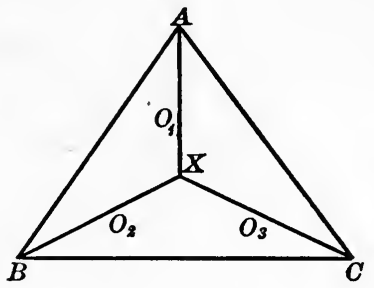

Let $A B C$ be any triangle, and $A O_{1}, B O_{2}, C O_{3}$, the bisectors of the angles.

It is required to prove that $A O_{1}, B O_{2}$, and $\mathrm{CO}_{3}$ meet in one point, or are concurrent.

Proof. Since $\angle \mathrm{s} A B O_{2}$ and $B A O_{1}$ are together less than two right angles [why?], $A O_{1}$ and $B O_{2}$ will meet.

Let them meet in $X$.

(Prop. XXV, Cor. III.)

Now $A O_{1}$ is the locus of points within the angle $B A C$ equidistant from $A B$ and $A C$.

(Prop. XXVIII.)

Also, $\mathrm{BO}_{2}$ is the locus of points within the angle $A B C$ equidistant from $A B$ and $A C$.

The point $X$ must therefore be equidistant from $A C$ and $B C$, and consequently must lie on $\mathrm{CO}_{3}$, which is the locus of points within the angle $A C B$ equidistant from $A C$ and $B C$.

Therefore $\mathrm{AO}_{1}, \mathrm{BO}_{2}, \mathrm{CO}_{3}$ meet in the point $\mathrm{X}$.

1. Produce the sides of the triangle and bisect two external angles and the third internal angle. Are these bisectors concurrent? Prove.

By this means we find four points $X, Y, Z, W$ associated with the triangle. Notice that when the figure is completed, these four points are joined in all possible ways, and that the line joining any two of them is perpendicular to the line joining the other two. 


\section{Proposition XXXVIII}

140. The perpendicular bisectors of the sides of a triangle are concurrent.

Let $A B C$ be any triangle; $D, E, F$, the mid-points of the sides.

It is required to prove that the perpendiculars erected at $D, E$, and $F$ meet in a point.

Proof. The perpendicular at $F$ is the locus of points equidistant from $A$ and $B$.

(Art. 72.)

The perpendicular at $E$ is the locus of points equidistant from $A$ and $C$.

These two perpendiculars intersect. Why?

Their point of intersection being equidistant from $B$ and $C$, must lie on the perpendicular at $D$.

Therefore the three perpendiculars meet in a point.

(Art. 74.)

\section{EXERCISES}

1. If from the points $A$ and $B$ of a straight line, and their mid-point $C$, perpendiculars are drawn to a given straight line, the perpendicular from $C$ is equal to the half-sum, or the half-difference of the perpendiculars from $A$ and $B$, according as $A$ and $B$ are on the same or on opposite sides of the given straight line.

2. If $D$ and $E$ are the mid-points of the sides $A B$ and $A C$ of a triangle $A B C$, and $B E$ is produced to $F$ making $E F$ equal to $B E$, and $C D$ is produced to $G$ making $D G$ equal to $C D$, show that $G, A$, and $F$ are in a straight line.

Suggestion. Show that $D E$ is parallel to both $A F$ and $A G$.

3. An angle of a triangle is right, acute, or obtuse according as the median drawn from its vertex is equal to, greater than, or less than half the side it bisects.

4. The feet of the four perpendiculars, drawn from one vertex of a triangle to the internal and external bisectors of the other two angles, lie on one straight line which passes through the mid-points of two sides of the triangle. 


\section{Proposition XXXIX}

141. The straight lines drawn from the vertices of a triangle perpendicular to the opposite sides are concurrent.

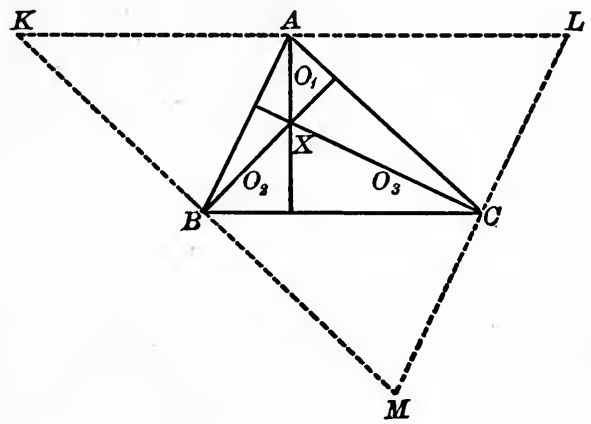

Let $A B C$ be any triangle, and from the three vertices draw the straight lines $A O_{1}, B O_{2}, C O_{3}$ perpendicular to the opposite sides.

It is required to prove that $A O_{1}, B O_{2}$, and $\mathrm{CO}_{3}$ are concurrent.

Proof. Through $A, B$, and $C$ draw the straight lines $K L$, $K M$, and $L M$ parallel respectively to the opposite sides of the triangle.

Then $A C B K$ is a parallelogram, as is also $A B C L$.

Hence $A$ is the mid-point of $K L$, and $A O_{1}$ is perpendicular to $K L$ since it is perpendicular to $B C$. (Prop. XXV., Cor. IV.)

Similarly $\mathrm{BO}_{2}$ is perpendicular to $K M$ at its mid-point.

And $\mathrm{CO}_{3}$ is perpendicular to $L M$ at its mid-point.

Therefore $\mathrm{AO}_{1}, \mathrm{BO}_{2}, \mathrm{CO}_{3}$ are concurrent. (Prop. XXXVIII.)

Definitron. The point $X$, in which the three perpendiculars from $A, B, C$, to the opposite sides intersect, is called the orthocentre of the triangle $A B C$. 


\section{EXERCISES}

1. If the point $D$ is the orthocentre of the triangle $A B C$, show that $A$ is the orthocentre of the triangle $B C D, B$, the orthocentre of the triangle $A C D$, and $C$, the orthocentre of the triangle $A B D$.

2. If the opposite sides of a quadrilateral are perpendicular to each other, the diagonals are also perpendicular to each other (Carnot's theorem). Could such a quadrilateral be convex ?

\section{Section V}

\section{AIDITIONAL PROBLEMS OF CONSTRUCTION}

142. In the preceding propositions we have introduced at convenient places several problems of construction, not because they form an indispensable part of the body of geometrical truth, but rather because of their inherent interest, and because they present to the beginner matters for proof which he clearly sees ought not to be accepted without proof.

By making use of the principles employed in the preceding problems the following can easily be solved:

1. To construct a triangle when two sides and the included angle are given.

2. To construct a triangle when a side and the two adjacent angles are given.

3. To construct a triangle when two angles and a side opposite to one of them are given.

This problem can easily be reduced to (2) by making use of the property of Proposition XXVII, Cor. I.

4. To construct a triangle when two sides and an angle opposite to one of them are given.

The pupil should notice all the cases that may arise in this problem according as the given angle is acute, obtuse, or a right angle; also according as the given angle is opposite the greater or the less of the two given sides; and should determine under what conditions there are two solutions, one solution, or no solution. 
5. To trisect a right angle.

Suggestion. Upon any segment $A C$ of one boundary, terminated at one end by the vertex, construct an equilateral triangle $A E C$. Similarly on an equal segment $A B$ construct an equilateral triangle $A D B . A D$ and $A E$ are the required trisectors. What lines might be omitted in the construction?

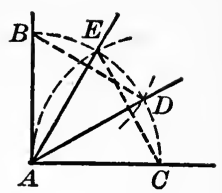

6. To trisect a given segment of a straight line.

Suggestion. Upon the given segment as base construct an equilateral triangle. Bisect the base angles. From the point where these bisectors meet, draw lines parallel to the sides.

\section{Typical Solutions}

143. We shall here insert a few typical problems with their solutions representing the principal methods of attack when a solution does not readily appear.

1. To construct a triangle having given one side, an adjacent angle, and the sum of the other two sides.

Let $a$ be equal to the given side, $b$ the sum of the other two sides, and $P$ the given angle.

Construction. Draw any line $A B$ equal to $a$. At one extremity $A$ construct an angle $B A C$ equal to $\angle P$, and make $A C$ equal to $b$. Now arises the problem to find a point $D$ in $A C$ such that if it be joined to $B, D B$ will equal $D C$, and so the sum of $A D$ and $D B$ will equal $A C$.

Assume the thing done. That is, choose some point $D$ and assume that it fulfils the given conditions.

Join $D B$ and $B C$. Then $B D C$ is an isosceles triangle, and a perpendicular from $D$ upon $B C$ would bisect $B C$.

But we were able to draw $B C$ without knowing $D$, also to bisect $B C$ and erect the perpendicular $E D$, and thus determine $D$.

lows :-

So the construction would be as fol-

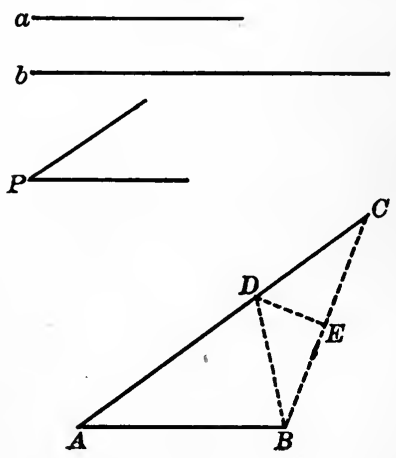


Draw $A B$ and $A C$ as before.

Join $B C$. Bisect $B C$ at $E$ and draw the perpendicular bisector cutting $A C$ at $D$. Join $D B$.

$\triangle A D B$ is the required triangle.

This method of solution is called Solving by Analysis.

Notice the order. We first assumed the required construction to have been made, then analyzed the diagram and sought to find upon what principles the construction rests and what steps would lead to it. Then by taking these steps in order the solution is accomplished.

2. Find a point in a given straight line, which is equidistant from two given points, (1) on the same side of the line, (2) on opposite sides of the line.

If $A$ and $B$ are the given points and $p$ the given straight line, the required point clearly is the intersection with $p$, of the perpendicular bisector of $A B$. For, this perpendicular bisector is the locus of points which are equidistant from $A$ and $B$.

Thus, by considering the locus of points satisfying given conditions we are led to the solution of the stated problem. This method may be called the method of Intersection of Loci.

3. Two points $A$ and $B$ are on opposite sides of a river whose banks are parallel straight lines. If the river must be crossed in a given direction, find the shortest path that can be taken from $A$ to $B$.

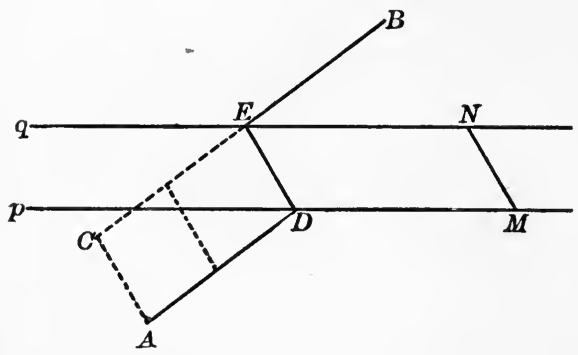

Let the parallel lines $p$ and $q$ represent the banks of the river, and $M N$ the direction in which the river must be crossed.

The line-segment $M N$ will indicate the distance that must be travelled in the given direction. 
From $A$ draw a line $A C$ equal and parallel to $M N$. Then $A C$ is the distance that must be travelled in the given direction; the remaining distance is to be as short as possible, and so will be equal to the linesegment $C B$.

Let $C B$ cut $q$ at $E$. Draw $E D$ parallel to $N M$ or $C A$, meeting $p$ at $D$. Join $A D$. Then $A D E B$ is the required path.

The pupil can easily prove that $A C E D$ is a parallelogram, and hence that the sum of the distances $A D, D E$, and $E B$ is equal to the sum of the distances $A C$ and $C B$. The distance travelled in going from $A$ to $B$ would have been the same if we had crossed from the line $A D$ to the parallel line $C B$, in the given direction, at any point, and the only problem was to shift the given distance $A C$ parallel to itself till it reached from one bank of the river to the other.

When a straight line is moved so as to remain always parallel to its original position it is said to be translated, and the motion is called a translation.

When any figure is moved so that all of its points describe parallel straight lines it is said to be translated.

Since the solution of this problem required the translation of the line $A C$, which satisfied some of the conditions of the problem into such a position as to satisfy the remaining conditions, the method is sometimes called the method of Translation.

\section{EXERCISES}

1. Through a given point to draw a straight line to make equal angles with two given intersecting lines.

Suggestion. Translate the bisector of the angle between the lines till it passes through the given point. See another solution on p. 66 .

2. Construct a triangle having given one side and the orthocentre.

3. Construct a triangle having given the base, the altitude, and the length of one side. Is the solution always possible?

4. Through a given point $P$ draw a straight line such that the perpendiculars to it from two fixed points meet it at equal distances from $P$.

5. Through a given point draw a line-segment terminated by two given intersecting straight lines, which shall be bisected at the given point.

6. Construct a right triangle having given the hypotenuse and the difference of the two sides.

7. Construct a triangle equiangular with a given triangle and having a given perimeter. 


\section{SECTION VI}

\section{SYMMETRICAL FIGURES}

144. If there be given any straight line $a$, and from a point $A$ we draw $A M$ perpendicular to the given line, and produce it to a point $A^{\prime}$ such that $M A^{\prime}$ equals $M A$, then $A$ and $A^{\prime}$ are called inverse points relative to the line $a$.

In the figure, $B$ and $B^{\prime}$ form another pair of inverse points relative to $a$.

Definition. If two figures are such that for every point of the one there is an inverse point in the other, relative to a certain straight line, the two figures are said to be symmetrical with respect to this line, and the line is called an axis of symmetry for the two figures.

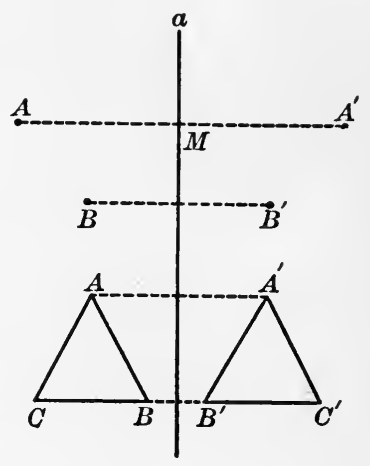

145. If you think of the plane in which there lie two figures symmetrical with respect to a certain straight line, as being folded along this line, just as you would fold a sheet of paper along a given straight line, it is clear that the two figures would come to coincide, since all lines perpendicular to the axis of symmetry would fold over upon themselves.

Hence,

Theorem. If two figures are symmetrical with respect to a straight line, they are superposable by inversion.

A figure symmetrical with a given figure can easily be obtained by tracing it with ink and then folding the paper along a straight line before the ink is dry. 
146. Two parts of the same figure may be symmetrical with respect to a given straight line, as in the diagram. In such cases we say that the figure is symmetrical with respect to the line.

Theorem. A circle is symmetrical with respect to any of its diameters.

Let $A P B$ be a circle whose centre is $O, A B$ any diameter, $P$ any point on the circle.

From $P$ draw $P M$ perpendicular to the diameter, and produce it to meet the circle again at $P^{\prime}$. Join $P O$ and $P^{\prime} O$.

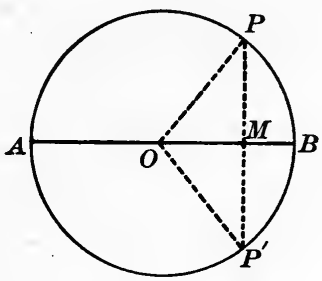

The $\triangle \mathrm{s} O P M$ and $O P^{\prime} M$ are identically equal. Hence $P^{\prime}$ is the inverse point of $P$, and for every point of the circle there is an inverse point with respect to the diameter.

\section{EXERCISES}

1. Show that a square is symmetrical with respect to each of its diagonals.

2. Show that an equiangular triangle is symmetrical with respect to each of its medians.

3. If a quadrilateral is symmetrical with respect to one of its diagonals, show that the two diagonals are at right angles.

4. If a triangle is symmetrical with respect to a median, it must be isosceles. 


\section{MISCELLANEOUS EXERCISES}

1. In a quadrilateral $A B C D$, if $A B$ is the greatest side and $C D$ the least, show that $\angle B C D$ is greater than $\angle D A B$, and $\angle C D A$ greater than $\angle A B C$.

2. Show that the sum of the diagonals of a convex quadrilateral is greater than the sum of either pair of opposite sides, and also greater than half the sum of the four sides.

3. The sum of the distances of any point within a rectilinear figure from the vertices is greater than half the sum of the sides.

4. If $D$ is any point on the side $B C$ of a triangle $A B C$, show that the sum of the sides of the triangle is greater than twice $A D$.

5. Show that no convex polygon can have more than three of its interior angles acute, nor more than three of its exterior angles obtuse.

6. On a given straight line find a point which is equidistant from two given lines. Under what conditions is the solution impossible?

7. Show how to find a point which is at a given distance from each of two intersecting straight lines. How many such points are there?

8. If two quadrilaterals are mutually equiangular, and two adjacent sides of the one are respectively equal to the two corresponding adjacent sides of the other, show that the quadrilaterals are identically equal.

9. Show that the sum of any two medians of a triangle is greater than the third median.

10. Show that the sum of the three medians of a triangle is greater than three-fourths of the sum of the sides of the triangle.

11. Show that if two of the medians of a triangle are equal, the triangle must be isosceles.

12. If $E$ and $F$ are the mid-points of the sides $A B$ and $C D$ of the parallelogram $A B C D$, show that the lines $E D$ and $B F$ will trisect the diagonal $A C$.

13. If the vertices of one parallelogram lie on the sides of another, show that all four diagonals pass through the same point.

14. If an exterior angle of a triangle be bisected, and also one of the interior non-adjacent angles, the angle made by the two bisectors is equal to half of the other interior non-adjacent angle. 
15. If two triangles on the same side of a common base have their sides which are terminated in opposite extremities of the base equal, the line joining the vertices will be parallel to the common base.

16. From the vertex $A$ of any triangle $A B C$ two straight lines are drawn meeting the opposite side at $D$ and $E$, so as to make the angle $B A D$ equal to the angle $C$, and the angle $C A E$ equal to the angle $B$. Show that the triangle $D A E$ is isosceles.

17. In any triangle $A B C$, the bisector of the angle $A$ makes with the perpendicular drawn from $A$ to the opposite side an angle equal to half the difference between the angles $B$ and $C$.

18. In any triangle $A B C$, the bisectors of the angles $B$ and $C$ make with each other an angle greater by a right angle than one-half $A$.

19. In any triangle $A B C$, the bisectors of the exterior angles at $B$ and $C$ make with each other an angle less by a right angle than one-half $A$.

20. In any convex quadrilateral, the bisectors of two consecutive angles make with each other an angle equal to the half-sum of the other two angles.

21. If through the point of intersection of the bisectors of the angles $B$ and $C$ in any triangle $A B C$, a line-segment $M N$ is drawn parallel to the side $B C$, and terminated by the sides $A B$ and $A C$, show that $M N$ equals the sum of $B M$ and $C N$.

22. If upon one boundary $O X$ of an angle you choose two points $A$ and $A^{\prime}$, and upon the other boundary $O Y$ you choose two points $B$ and $B^{\prime}$, such that $O B$ equals $O A$ and $O B^{\prime}$ equals $O A^{\prime}$, and join crosswise $A B^{\prime}$ and $A^{\prime} B$, show that the intersection of these lines is upon the bisector of the angle $X O Y$.

23. If two sides of a triangle are unequal, the median through their intersection makes the greater angle with the lesser side.

24. A quadrilateral which has two sides equal and the other two sides parallel may be a parallelogram, or it may be a trapezoid having an axis of symmetry.

25. In an isosceles triangle the sum of the distances of a point in the base from the two sides is the same, no matter where in the base the point is chosen.

If the point should be chosen in the base produced, how would this theorem be altered?

26. In an equilateral triangle the sum of the distances of a point within the triangle from the three sides is the same, no matter where the point is chosen. 


\section{SUMMARY OF CHAPTER I}

1. Definitions.

(1) Scalene Triangle, Isosceles Triangle, Equilateral Triangle. See $\S 33$.

(2) Right Triangle - a triangle having one right angle. $\$ 61$.

(3) Hypotenuse of a Right Triangle - the side opposite the right angle. $\S 61$.

(4) Complementary Angles - two angles whose sum is equal to a right angle. $\S 102$.

(5) Corollary - a theorem, the truth of which is easily deduced from another, or the proof of which is easily deduced from the proof of another. $\S 50$.

(6) Converse Theorems - two theorems such that the hypothesis of each is the conclusion of the other. $\S 44$.

(7) Locus - a line or group of lines such that all their points satisfy certain conditions while no other points satisfy those conditions. $\S 73$.

(8) Parallel Lines - straight lines lying in the same plane, and such that if they are drawn ever so far either way they will not intersect. $\S 85$.

(9) Polygon - a closed rectilinear figure of any number of sides. $\S 114$.

(10) Convex Polygon - one which lies wholly on one side or the other of each of its sides. $\S 115$.

(11) Diagonal of a Polygon - a straight line joining any two nonconsecutive vertices. $\S 109$.

(12) Quadrilateral - a closed rectilinear figure having four sides. $\S 114$.

(13) Parallelogram - a quadrilateral whose opposite sides are parallel. $\S 119$.

(14) Trapezoid - a quadrilateral having one and only one pair of opposite sides parallel. $\$ 119$.

(15) Isosceles Trapezoid - a trapezoid having its non-parallel sides equal. Ex. 7, p. 77.

(16) Trapezium - a quadrilateral having no two sides parallel. §119.

(17) Rhombus - a parallelogram having two adjacent sides equal. $\S 120$.

(18) Rectangle - a parallelogram having one right angle. \$121.

(19) Square - a parallelogram having one right angle and a pair of adjacent sides equal. $\S 122$. 
(20) Perimeter of a Polygon - the sum of its sides. $§ 132$.

(21) Median of a Triangle - a straight line drawn from any vertex to the mid-point of the opposite side. $\S 134$.

(22) Centroid of a Triangle - the point at which its medians intersect. $\S 137$.

(23) Concurrent Lines - straight lines which pass through the same point. $\S 135$.

(24) Inverse Points - two points are inverse relative to a given straight line when the line-segment joining them is bisected perpendicularly by the given line. $\S 144$.

(25) Symmetrical Figures - a figure is symmetrical relative to a given straight line when for every point of the figure there is an inverse point of the figure relative to that line. $\S 144$.

Two figures are symmetrical relative to a given straight line when for every point of one figure there is an inverse point of the other, relative to that line. $\S 144$.

(26) Axis of Symmetry - a straight line relative to which a figure is symmetrical. $\S 144$.

2. Axioms.

Two magnitudes $A$ and $B$ of the same kind are either equal, or $A$ is greater than $B$, or $A$ is less than $B$ (Axiom 10). $\$ 64$.

3. Postulates.

Through any point one and only one straight line can be drawn parallel to a given straight line (Postulate 5). $\quad \S 86$.

4. Problems.

(1) To construct an equilateral triangle, having given the length of each side. $\S 34$.

(2) To construct an isosceles triangle, having given the lengths of the sides. $\$ 37$.

(3) To construct any triangle, having given the lengths of the sides. $\S 39$.

(4) To bisect a given angle. $\$ 54$.

(5) To bisect a given line-segment. $\$ 56$.

(6) From a given point in a straight line to draw a perpendicular to the line. $\$ 71$.

(7) From a given point without a given straight line, to draw a perpendicular to the line. $\S 75$.

(8) At a given point on a given straight line to construct an angle equal to a given angle. $\S 84$. 
(9) Through a given point to draw the straight line parallel to a given straight line. $\S 99$.

(10) To construct a triangle having given one side, an adjacent angle, and the sum of the other two sides. $\$ 143.1$.

(11) In a given straight line to find a point equidistant from two given points. $§ 143.2$.

(12) To find the shortest path between two given points when a part of the path is given both in length and direction. $\$ 143.3$.

5. Theorems on Bisectors.

(1) Through the vertex of an angle there can be drawn one and only one straight line which bisects the angle. $\S 46$.

(2) In a given line-segment there can be found one and only one point which bisects the line-segment. $\S 47$.

(3) Two points each equidistant from the extremities of a given line-segment determine the perpendicular bisector of the linesegment. $\S 57$.

(4) The bisector of an angle is the locus of points within the angle which are equidistant from its boundaries. $\S 107$.

6. Theorems on Perpendiculars.

(1) At any point in a straight line there can be drawn one, and only one, perpendicular to the line. $\$ 46$.

(2) From a point outside a given straight line not more than one perpendicular to the line can be drawn. $\S 62$.

(3) Perpendiculars to the same straight line, lying in the same plane, are parallel. $\$ 91$.

(4) If a straight line is perpendicular to one of two parallel lines, it is perpendicular also to the other. $\S 97$.

(5) If two straight lines intersect, lines perpendicular to them in the same plane will also intersect. $§ 98$.

7. Theorems on tile Equality of Triangles.

Two triangles are identically equal if they have-

(1) Two sides and the included angle of one equal, respectively, to two sides and the included angle of the other. $\S 41$.

(2) A side and the two adjacent angles of one equal, respectively, to a side and the two adjacent angles of the other. $\$ 43$.

(3) The three sides of one equal, respectively, to the three sides of the other. $\$ 53$.

(4) Each a right angle, and the hypotenuse and a side of one equal, respectively, to the hypotenuse and a side of the other. $\$ 78$. 
(5) Two sides of one equal, respectively, to two sides of the other and the angles opposite a pair of equal sides also equal $\S \S 81,82$.

(6) Two angles of one equal, respectively, to two angles of the other, and the sides opposite one pair of equal angles also equal. $\S 106$.

\section{Theorems on Unequal Triangles.}

(1) If two triangles have two sides of the one equal, respectively, to two sides of the other, and the included angles unequal, the triangle which has the greater included angle has also the greater third side. $\S 79$.

(2) If two triangles have two sides of the one equal, respectively, to two sides of the other, and the third sides unequal, the triangle which has the greater third side has also the greater included angle. $\$ 80$.

9. Theorems on the Properties of Triangles.

(1) In an isosceles triangle, the angles opposite the equal sides are equal. $\S 48$.

(2) The straight line which bisects the vertical angle of an isosceles triangle also bisects the base, and is perpendicular to the base. $\$ 49$.

(3) An equilateral triangle is also equiangular. $\$ 50$.

(4) If two angles of a triangle are equal, the sides opposite those angles are also equal. $\S 51$.

(5) An equiangular triangle is also equilateral. $\$ 52$.

(6) If one side of a triangle is produced, the exterior angle so formed is greater than either of the two interior non-adjacent angles. $\S 59$.

(7) Any two angles of a triangle are together less than two right angles. $\S 60^{\circ}$.

(8) If a triangle has one right angle or one obtuse angle, the other two angles must be acute. $\$ 61$.

(9) If two sides of a triangle are unequal, the angle opposite the greater side is greater than the angle opposite the less. $\$ 63$.

(10) If two angles of a triangle are unequal, the side opposite the greater angle is greater than the side opposite the less. $\S 65$.

(11) In a right triangle the hypotenuse is the greatest side. $\$ 66$. 
(12) Any two sides of a triangle are together greater than the third side. $\$ 70$.

(13) If one side of a triangle is produced, the exterior angle so formed is equal to the sum of the two interior non-adjacent angles. $\S 100$.

(14) The sum of the three angles of a triangle is equal to two right angles. $\S 101$.

(15) The line-segment joining the mid-points of two sides of a triangle is parallel to the third side and equal to half of it. $\S 130$.

(16) If from the mid-point of one side of a triangle there is drawn a straight line parallel to a second side, this line passes through the mid-point of the third side. $\$ 133$.

(17) The medians of a triangle are concurrent. $\$ 136$.

(18) The centroid of a triangle is a trisection point of each median. $\S 138$.

(19) The bisectors of the angles of a triangle are concurrent. $\S 139$.

(20) The perpendicular bisectors of the sides of a triangle are concurrent. $\$ 140$.

(21) The straight lines drawn from the vertices of a triangle perpendicular to the opposite sides are coneurrent. $§ 141$.

10. Theorems on Parallel Lines.

(1) If two straight lines lying in one plane are such that a transversal makes a pair of alternate angles equal, or any pair of corresponding angles equal, these two lines are parallel. $\$ \S 88,89$.

(2) If two straight lines lying in one plane are such that a transversal makes a pair of interior angles, or a pair of exterior angles, on the same side of it supplementary, these two lines are parallel. $\S 90$.

(3) Two straight lines parallel to the same straight line are parallel to each other. $\$ 92$.

(4) If two parallel straight lines are cut by a transversal, any two alternate angles, and any two corresponding angles, are equal. $\S \S 93,94$.

(5) If two parallel straight lines are cut by any transversal, the interior angles, and also the exterior angles, on the same side, are supplementary. $\S 95$.

11. Tireorems on Convex Polygons.

(1) The sum of the interior angles of any convex polygon, together with four right angles, makes up twice as many right angles as the polygon has sides. $§ 116$. 
(2) If the sides of a convex polygon are produced in order, the exterior angles so formed are together equal to four right angles. $§ 117$.

12. Theorems on Parallelograms.

(1) The opposite sides and the opposite angles of a parallelogram are equal. $\$ 123$.

(2) Either diagonal of a parallelogram divides the figure into two superposable triangles. $\$ 124$.

(3) The diagonals of a parallelogram bisect each other. $\S 126$.

(4) If two sides of a quadrilateral are parallel and equal, the figure is a parallelogram. $\$ 127$.

(5) If both pairs of opposite sides of a quadrilateral are equal, the figure is a parallelogram. $\S 128$.

(6) If two parallelograms have two adjacent sides and the included angle of one equal, respectively, to two adjacent sides and the included angle of the other, the parallelograms are identically equal. $\$ 129$.

13. Miscellaneous Theorems.

(1) If two straight lines intersect, the vertical angles are equal. $\$ 58$.

(2) Not more than two equal line-segments can be drawn from a given point to a given straight line. $\$ 67$.

(3) If any pair of alternate angles formed by two straight lines and a transversal are unequal, or if any pair of corresponding angles are unequal, or if the interior angles on one side are not supplementary, the two lines are not parallel, and therefore will meet if produced. $\$ 96$.

(4) If the boundaries of one angle are respectively parallel or perpendicular to the boundaries of another, these two angles are either equal or supplementary. $\S 105$.

(5) If the mid-points of the sides of any quadrilateral be joined in order, the figure so formed is a parallelogram, and the sum of the sides of this parallelogram equals the sum of the diagonals of the quadrilateral. $\S 131$.

14. On Symmetry.

(1) If two figures are symmetrical with respect to a straight line, they are superposable by inversion. $\$ 145$.

(2) A circle is symmetrical with respect to any of its diameters. $\S 146$. 


\section{CHAPTER II}

\section{THE CIRCLE}

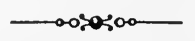

\section{SeCtion I}

\section{DEFINITIONS AND PRELIMINARY THEOREMS}

147. In the introductory chapter the following definitions were given :

A circle is a closed line all points of which are equally distant from a certain point within it called the centre.

The line-segment joining the centre to any point of the circle is called a radius, and a line-segment through the centre terminated both ways by the circle is called a diameter.

From these definitions it follows that all radii of the same circle are equal, and all diameters of the same circle are equal.

The distance from the centre of any point inside the circle is less than a radius, and of any point outside the circle is greater than a radius.

148. Theorem I. Two circles in a plane which have the same centre and equal radii coincide throughout.

For, if there is any point of one circle which does not coincide with a point of the other, it must lie either inside or outside of the other, and hence its distance from the centre is either less or greater than a radius of the other. But this is not the case, since all radii of the same circle are equal by definition and radii of the two circles are assumed to be equal.

Corollary I. Two circles in a plane which have the same centre and one point in common coincide throughout. 
For they have the same centre and equal radii, since the line joining their common point to the centre is a radius of each circle.

Corollary II. Two circles which have equal radii can be made to coincide, and hence are identically equal; and conversely, equal circles have equal radii.

149. Theorem II. Through a given point any number of different circles can be described.

For, if $A$ is the given point, we may choose any point $O$ whatever for centre, and with radius $O A$ describe a circle which passes through $A$.

Since a circle is a closed curve, two circles which intersect at one point must also intersect at a second point.

(Art. 25.)

150. Theorem III. Through two given points any number of different circles can be described.

Let $A$ and $B$ be the given points; then every point of the perpendicular bisector of the line-segment $A B$ is equidistant from $A$ and $B$ (Art. 72). Choose any point $O$ on this perpendicular bisector for centre, and the circle described with radius $O A$ will pass through both $A$ and $B$.

151. Theorem IV. Through three given points not in the same straight line, one and only one circle can be described.

Let $A, B$, and $C$ be the three given points. Then the locus of points equidistant from $A$ and $B$ is the perpendicular bisector of the line-segment $A B$, and the locus of points equidistant from $B$ and $C$ is the perpendicular bisector of the line-segment $B C$. These two loci have one (Art. 98) and only one common point; which point, $O$ say, is equidistant from $A, B$, and $C$, and is the only such point.

With $O$ as centre and with radius $O A$ a circle may be described through $A, B$, and $C$, while no other circle can be 
described through these three points, since no other point than $O$ can be found for centre.

Corollary I. Two circles which coincide at three points coincide throughout.

For they must have the same centre and equal radii.

Corollary II. Two different circles can have at most two points in common;

Or, two circles can intersect in at most two points.

Incidentally in this article we have solved the problem:

To find the centre of the circle which passes through three given points, or of which at least three points are given.

This is the same problem as To pass a circle through the three vertices of a triangle, a thing which can always be done.

152. The questions arise:

Can a circle be described through three given points which lie in a straight line?

If you should proceed as in the case where the three points do not lie in one straight line, in what particular would the construction fail ?

How many points of a straight line are equidistant from any given point?

(See Art. 67.)

Theorem V. A straight line can intersect a circle in at most two points;

Or, what is the same thing, a straight line can have at most two points in common with any circle.

153. Definitions. Any portion of a circle terminated by two points is called an arc of the circle.

The straight line joining any two points of a circle, i.e. joining the extremities of an arc, is called a chord of the circle. 
The chord is said to subtend the are, the arc to be subteraied by the chord.

Every chord subtends two ares of the circle, one on either side of it, called the greater or major arc and the lesser or minor arc.

The two arcs subtended by any chord together make up the whole circle. Each of these ares is called the conjugate of the other.

When we speak of the arc subtended by a given chord we shall always have in mind the lesser or minor are unless the contrary is expressly stated.

154. The arc of a circle subtended by a diameter is called a semicircle.

Since a circle is symmetrical with respect to any of its diameters (Art. 146), a semicircle and its conjugate semicircle are equal. If you should fold the circle over, along the diameter, the two semicircles would coincide.

Hence any semicircle is half of a circle, and all semicircles belonging to the same or equal circles are equal.

155. Theorem VI. The perpendicular bisector of any chord of a circle passes through the centre.

For it is the locus of points equidistant from the extremities of the chord, and the centre being equidistant from the extremities of the chord must lie on this locus.

156. Definitions. The figure formed by an arc of a circle and its subtending chord is called a segment of the circle.

The figure formed by an arc and the two radii to its extremities is called a sector of the circle. The angle at the centre formed by two radii is called the angle at the centre subtended by the intercepted arc, or by the chord of that arc.

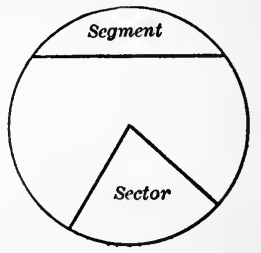




\section{Proposition I}

157. If in equal circles two angles at the centre are equal, the arcs subtending them are also equal; and of two unequal angles at the centre, the greater is subtended by the greater arc.
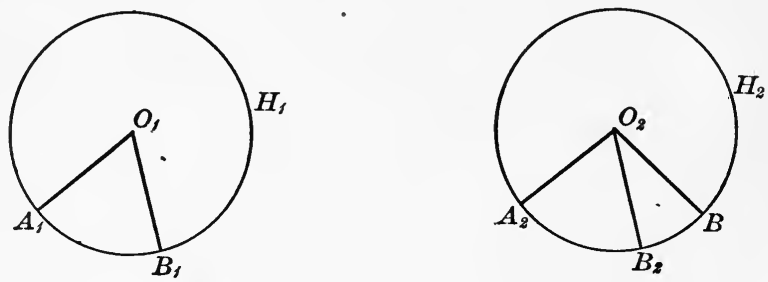

In the equal circles $A_{1} B_{1} H_{1}$ and $A_{2} B_{2} H_{2}$, of which the centres are $O_{1}$ and $O_{2}$,

First, let the angle $A_{1} O_{1} B_{1}$ be equal to the angle $A_{2} O_{2} B_{2}$.

It is required to prove that the arc $A_{1} B_{1}$ is equal to the are $A_{2} B_{2}$.

Proof. Superpose the circle $O_{1}$ on the circle $O_{2}$ so that their centres coincide. Then the circles will coincide throughout, since by hypothesis they are equal, and every point of the one will coincide with a point of the other.

Further, make $\angle A_{1} O_{1} B_{1}$ coincide with its equal $\angle A_{2} O_{2} B_{2}$.

Since the radii of the two circles are equal, $A_{1}$ will coincide with $A_{2}$, and $B_{1}$ with $B_{2}$, so that the arc $A_{1} B_{1}$ will coincide with and be equal to the arc $A_{2} B_{2}$.

Next, let the angle $A_{1} O_{1} B_{1}$ be greater than the angle $A_{2} O_{2} B_{2}$.

It is required to prove that the arc $A_{1} B_{1}$ is greater than the $\operatorname{arc} A_{2} B_{2}$.

Proof. As before, superpose the circle $O_{1}$ on the circle $O_{2}$ so that their centres coincide, and so that the radius $O_{1} A_{1}$ coincides with the radius $O_{2} A_{2}$. 
Since $\angle A_{1} O_{1} B_{1}$ is greater than $\angle A_{2} O_{2} B_{2}$, the radius $O_{1} B_{1}$ will not coincide with $\mathrm{O}_{2} B_{2}$, but will fall outside $\angle A_{2} O_{2} B_{2}$ in some position such as $O_{2} B$.

The are $A_{1} B_{1}$, which will coincide with the arc $A_{2} B$, will therefore overlap and be greater than the arc $A_{2} B_{2}$.

158. Conollary. If in the same circle two angles at the centre are equal, the arcs subtending them are also equal; and of two unequal angles at the centre, the greater is subtended by the greater arc.

\section{Proposition II}

159. If in equal circles two arcs are equal, the angles at the centre which they subtend are also equal; and of two unequal arcs the greater subtends the greater angle at the centre.

[This theorem is the converse of the theorem in Proposition I, and may be proved either indirectly or by superposition. The pupil should write out the proof by both methods.]

160. Corollary. If in the same circle two arcs are equal, the angles at the centre which they subtend are also equal; and of two unequal arcs, the greater subtends the greater angle at the centre.

\section{EXERCISES}

1. Every point of a chord of a circle, except its extremities, lies inside the circle.

Let $O$ be the centre of the circle, $A$ and $B$ the extremities of the chord, $E$ any other point of the chord. Prove that $O E$ is less than $O A$.

2. Find the centre of a circle having given any arc of it.

3. What is the locus of the centres of circles passing through two given points ?

4. Describe a circle that shall pass through two given points and have a given radius. 


\section{Proposition III}

161. If in the same circle or in equal circles two arcs are equal, the chords subtending them are also equal; and of two unequal minor arcs, the greater is subtended by the greater chord.
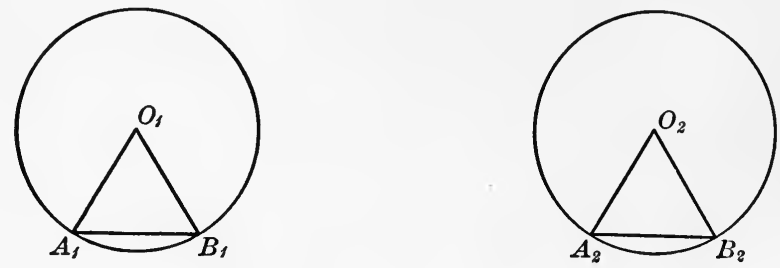

Let the pupil give a particular enunciation of the theorem, applying it to the diagram.

Suggestions for Proof. First, ( $a$ ) equal ares subtend equal angles at the centre.

(Prop. II.)

(b) $\triangle \mathrm{s} O_{1} A_{1} B_{1}$ and $O_{2} A_{2} B_{2}$ are identically equal. (Art. 41.)

(c) Therefore the chord $A_{1} B_{1}$ equals the chord $A_{2} B_{2}$.

Next, $(a)$ the greater arc subtends the greater angle at the centre.

(Prop. II.)

(b) Apply Proposition XX, Ch. I, to the $\triangle \mathrm{s} O_{1} A_{1} B_{1}$ and $\mathrm{O}_{2} A_{2} B_{2}$.

Therefore . . .

162. Corollary. If in the same circle, or in equal circles, two angles at the centre are equal, the chords subtending them are also equal; and of two unequal angles at the centre, both less than straight angles, the greater is subtended by the greater chord.

EXERCISE. - Why is it necessary in this proposition and its corollary to assume that the unequal arcs are minor arcs, and that the unequal angles are less than straight angles? 


\section{Proposition IV}

163. If in the same circle, or in equal circles, two chords are equal, the arcs subtended by them are also equal; and of two unequal chords, the greater subtends the greater minor arc.

164. Corollary. If in the same circle, or in equal circles, two chords are equal, the angles at the centre which they subtend are also equal; and of two unequal chords, the greater subtends the greater angle.

\section{Proposition V}

165. The straight line drawn from the centre of a circle perpendicular to a chord bisects the chord, and if produced, bisects the arc subtended by the chord.

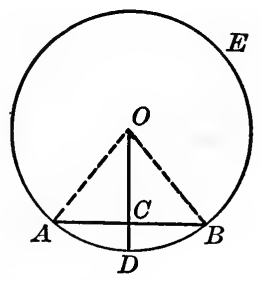

Let $A B$ be any chord of the circle $A E B$ whose centre is $O$, and $O C$ a line drawn from $O$ perpendicular to $A B$.

It is required to prove that $O C$ bisects the chord $A B$ at $C$, and if produced, bisects the arc $A B$ at $D$.

Proof. Join $O A$ and $O B$.

$\triangle \mathrm{s} O A C$ and $O B C$ are identically equal. Why?

Therefore

$$
A C=B C \text {. }
$$

Also

$\angle A O D=\angle B O D$.

Therefore

$$
\operatorname{arc} A D=\operatorname{arc} B D \text {. }
$$


166. CoRollary I. The straight line drawn from the centre of a circle to the mid-point of a chord is perpendicular to the chord.

167. Corollary II. The mid-points of a system of parallel chords all lie on a diameter perpendicular to the chords.

For the diameter drawn to the mid-point of one chord is perpendicular to that chord (Cor. 1), and therefore to every chord of the system (Art. 97). Hence this diameter passes through the mid-point of every chord of the system.

168. Corollary III. The straight line joining the centres of two intersecting circles bisects their common chord at right angles.

Suggestion. Join the mid-point of the common chord with the centre of one circle. This line, if produced backward, will pass through the centre of the other circle. Why?

169. Corollary IV. The diameter which bisects a chord also bisects the angle at the centre subtended by the chord.

\section{EXERCISES}

1. If two chords of a circle intersect, they cannot both be bisected at their common point, unless that point is the centre.

2. Through a given point within a circle, not the centre, draw a chord which is bisected at that point. Is there more than one such chord?

3. Prove that the arcs of a circle which lie between two parallel chords are equal.

4. The straight line joining the mid-point of an arc of a circle and the mid-point of its chord is perpendicular to the chord, and will, if produced, pass through the centre of the circle.

5. The diameter which bisects an arc of a circle also bisects its chord at right angles.

6. Show how to bisect a given arc of a circle.

7. If a quadrilateral is inscribed in a circle, the perpendiculars to the sides at their mid-points meet in one point. 


\section{Proposition VI}

170. In the same circle, or in equal circles, equal chords are equidistant from the centre; and of two unequal chords, the greater is nearer to the centre than the less.
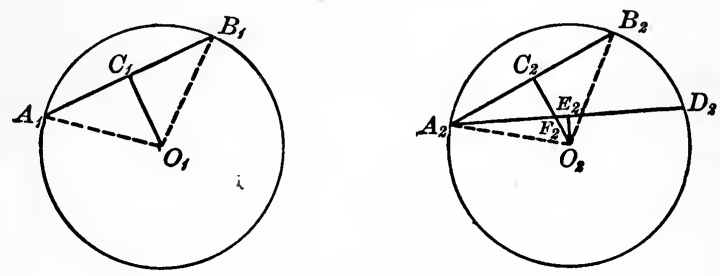

First, let $O_{1}$ and $O_{2}$ be the centres of two equal circles, $A_{1} B_{1}$ and $A_{2} B_{2}$ equal chords in them.

It is required to prove that the perpendiculars $O_{1} C_{1}$ and $\mathrm{O}_{2} \mathrm{C}_{2}$ from the centre upon the chords are equal.

Proof. The angles at the centre $A_{1} O_{1} B_{1}$ and $A_{2} O_{2} B_{2}$ are equal. Give reasons. Also $\angle A_{1} O_{1} C_{1}=\angle A_{2} O_{2} C_{2}$. Why?

$\triangle A_{1} O_{1} C_{1}$ is identically equal to $\triangle A_{2} O_{2} C_{2}$. Prove.

Therefore

$$
O_{1} C_{1}=O_{2} C_{2} \text {. }
$$

Next, let the chord $A_{2} D_{2}$ be greater than the chord $A_{2} B_{2}$.

It is required to prove that the perpendicular $\mathrm{O}_{2} \mathrm{C}_{2}$ upon the lesser chord is greater than the perpendicular $O_{2} E_{2}$ upon the greater chord.

Proof. Since the chord $A_{2} D_{2}$ is greater than the chord $A_{2} B_{2}$, the arc $A_{2} D_{2}$ is greater than the arc $A_{2} B_{2}$.

(Prop. IV.)

If the chords be so placed as to coincide at one extremity and lie on the same side of the centre, the arc $A_{2} D_{2}$ will extend beyond the arc $A_{2} B_{2}$, and the chord $A_{2} B_{2}$ will lie on the opposite side of $A_{2} D_{2}$ from the centre. Consequently, the perpendicular $\mathrm{O}_{2} \mathrm{C}_{2}$ will intersect the chord $A_{2} D_{2}$ at some point $F_{2}$.

Now $O_{2} C_{2}$ is greater than $O_{2} F_{2}$, and $O_{2} F_{2}$ is greater than $O_{2} E_{2}$. Why? Therefore $\mathrm{O}_{2} \mathrm{C}_{2}$ is greater than $\mathrm{O}_{2} E_{2}$. 


\section{Proposition VII}

171. In the same circle, or in equal circles, chords equidistant from the centre are equal; and of two chords unequally distant, the one nearer the centre is the greater.

What is the relation of this proposition to Proposition VI?

The proof is left to the pupil, with the suggestion that the indirect method will probably be easiest.

Thus, if in the first case, the chords are not equal, one or the other of them must be nearer the centre. Which? Prop. VI.

In the second case, if the one nearer the centre is not the greater, what?

172. Corollary. A diameter is the greatest chord that can be drawn in any circle.

This follows directly from the above proposition, or it may be shown otherwise, as follows :-

The diameter $A B$ equals the sum of the radii $A O$ and $O C$, which is greater than any chord $A C$, not a diameter.

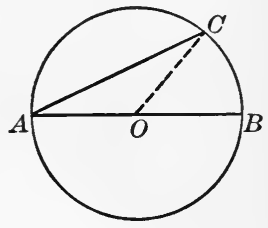

\section{EXERCISES}

1. If an equilateral triangle is inscribed in a circle, the sides are equidistant from the centre.

2. If two chords of a circle which intersect make equal angles with the line joining their common point to the centre, show that the chords are equal.

3. A chord which is perpendicular to a radius is less than any other chord through their point of intersection.

4. If from a point within a circle more than two equal straight lines can be drawn to the circle, that point must be the centre.

Suggestion. - Suppose that three equal lines can be drawn from the point, and show that the point is the intersection of two lines upon each of which the centre lies. See Art. 155 .

5. Describe two concentric circles each of which passes through two given points, the first through $A$ and $B$, say, and the second through $C$ and $D$. 


\section{Proposition VIII}

173. Of all line-segments which can be drawn to a circle from a point within it, not the centre, the greatest is that which passes through the centre, and the least is that which, if produced backward, would pass through the centre; and of any two others, the greater is that which makes the less angle with the greater segment of the diameter through the point.

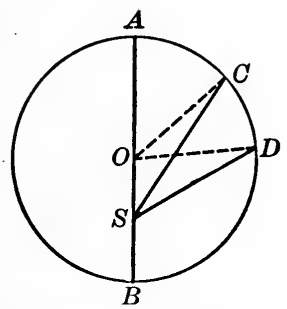

Let $S$ be any point within a circle other than the centre, $S A$ the line drawn from $S$ through the centre to the circle, $S B$ the line from $S$ to the circle which, if produced backward, would pass through the centre, $S C$ and $S D$ any other straight lines from $S$ to the circle, of which $S C$ makes a less angle with the diameter through $S$ than does $S D$.

It is required to prove (1) that $S A$ is the greatest line from $S$ to the circle, (2) that $S B$ is the least line, and (3) that $S C$ is greater than $S D$.

Proof. Join $O C$ and $O D$.

First, $S A$ equals the sum of $S O$ and $O C$.

But the sum of $S O$ and $O C$ is greater than $S C$. Why?

Therefore $S A$ is greater than $S C$, any other line drawn from $S$ to the circle.

Next, $S B$ equals the difference between $S O$ and $O C$.

But the difference between $S O$ and $O C$ is less than $S C$. (Ex. 3, p. 48.) 
Therefore $S B$ is less than $S C$, any other line drawn from $S$ to the circle.

Lastly, if we rotate the line $S C$ about $S$ into the position $S D$, thereby increasing $\angle A S C$ (hypothesis), we at the same time increase $\angle A O C$ till it becomes $\angle A O D$.

Since $\angle A O C$ is less than $\angle A O D$, the supplementary $\angle S O C$ is greater than the supplementary $\angle S O D$, while the sides containing these angles are respectively equal.

Therefore $S C$ is greater than $S D$.

174. Corollary. From any point within a circle two equal straight lines can be drawn to the circle; these make equal angles with the diameter through the point.

\section{EXERCISES}

1. State and prove a theorem for a point without a circle similar to that of Proposition VIII.

2. If two circles intersect, any two parallel lines drawn through the points of intersection and terminated both ways by the circles are equal.

3. If two circles intersect, any two lines drawn through one point of intersection, making equal angles with the line of centres and terminated both ways by the circles, are equal.

4. If with the vertex of an isosceles triangle for centre a circle is described which cuts the base or the base produced, show that the segments of the base line intercepted between the extremities of the base and the circle are equal.

175. In Proposition VIII we saw that if from $S$, any point within a circle, not the centre, a straight line $S P$ is drawn to the circle, and the line rotated about $S$ in the way indicated by the arrowhead, while $P$ traverses the circle, the magnitude $S P$ will continuously increase till $P$ reaches the point $A$. After this it will continuously decrease till $P$ comes to coincide with $B$, when it will again begin to increase.

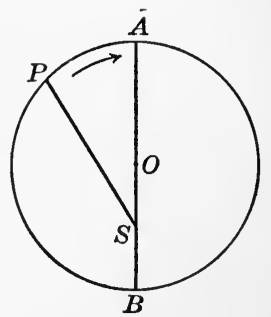


The magnitude $S P$ is thus a variable quantity, varying continuously as the angle $A S P$ varies continuously. It has a maximum value, viz. $S A$, and a minimum value, viz. $S B$.

If the point $S$ is chosen on the circle, $S P$ is a variable chord whose maximum value is a diameter and whose minimum value is zero.

\section{Section II}

\section{ANGLES INSCRIBED IN ARCS}

176. Definition. If a point is chosen on any arc of a circle, and the chords are drawn from it to the extremities of the arc, the angle between these chords is said to be an angle in the arc, or an angle inscribed in the arc, and the arc is said to contain the angle.

An angle in an arc is often spoken of as an angle in the segment formed by the arc and its chord, and the segment then is said to contain the angle.

The angle $A C B$ is said to be inscribed in the arc $A E B$, or in the segment $A E B$; it is an angle at a point of the circle subtended by the arc $A F B$, or subtended by the chord $A B$.

Sometimes the expression 'an angle stands upon an arc' is used instead of 'is subtended by an arc.'

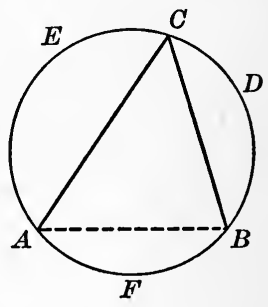

ExERcise. In what are is $\angle A B C$ inscribed? What arc does it subtend? What chord does it subtend?

177. Definition. A polygon is said to be inscribed in a circle when its vertices lie on the circle; and the circle is said to be circumscribed about the polygon.

Each angle of an inscribed polygon is subtended by an arc of the circle, and is inscribed in the conjugate arc. 


\section{Proposition IX}

178. An angle whose vertex is on a circle equals half the angle at the centre subtended by the same arc.

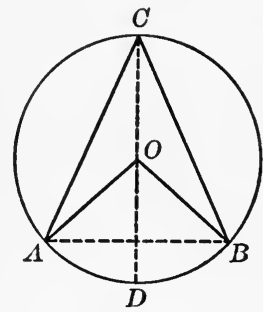

FIG. 1

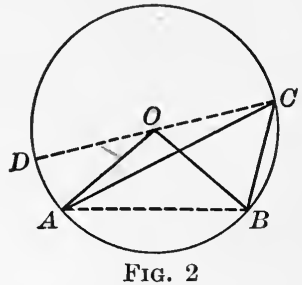

FIG. 2

Let $A B$ be any arc of a circle, $A C B$ an angle at the point $C$ of the circle, and $A O B$ the angle at the centre subtended by the same arc $A B$.

It is required to prove that the angle $A C B$ equals half the angle $A O B$.

Proof. Draw $C O$ and produce it to meet the circle at $D$.

$$
\angle O C A=\angle O A C \text {. }
$$

And $\angle A O D$ equals the sum of $\angle O A C$ and $O C A$. (Art. 100.) Therefore $\angle A C O$ equals half of $\angle A O D$.

Similarly $\angle B C O$ equals half of $\angle B O D$. Prove.

Therefore the sum (Fig. 1), or difference (Fig. 2), of $\angle A C O$ and $B C O$ equals half the sum, or difference, of $\angle A O D$ and $B O D$.

That is, $\angle A C B$ equals half of $\angle A O B$.
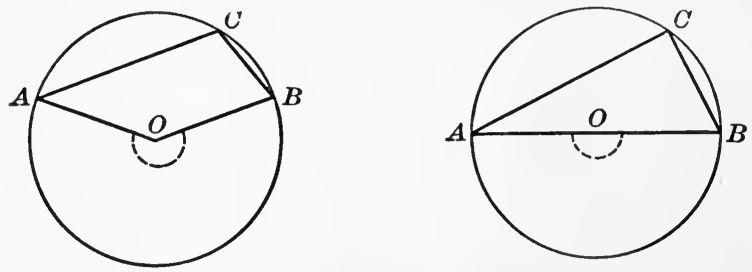

If the arc subtending the angle is greater than a semicircle, then the angle at the centre becomes the reflex angle $A O B$; 
and if the arc is a semicircle, the angle at the centre becomes the straight angle $A O B$. The same method of proof will show that the theorem holds in these cases as well as in the cases treated in the proposition.

179. Corolla Ry I. An angle in a semicircle is a right angle.

For it is half of a straight angle. Prove also by joining the centre with the vertex $C$.

180. Corollary II. An angle in an arc greater than a semicircle is acute, and an angle in an arc less than a semicircle is obtuse.

181. Corollary III. All angles in a circle subtended by the same arc, or by equal arcs, are equal ;

Or in other words, all angles inscribed in the same segment, or in equal segments, of a circle are equal.

For they are all equal to half of the same angle at the centre of the circle.

182. Corollary IV. Equal angles on the same base, and on the same side of it, have their vertices on an arc of a circle of which the given base is the chord.

If $A C B$ and $A D B$ are equal angles on the same side of $A B$, the circle passing through $A, C$, and $B$, must also pass through $D$. For if not it will cut $A D$ at some other point as $E$. Then $\angle A E B$ will equal $\angle A C B$.

(Cor. III.)

But $\angle A E B$ is greater (or less) than $\angle A D B$.

(Art. 59.)

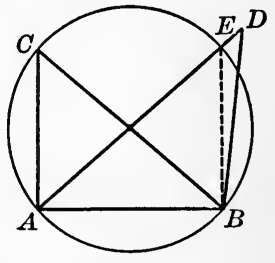

Therefore $\angle A C B$ is not equal to $\angle A D B$, contrary to hypothesis.

Therefore, etc.

183. Corollary V. The circle described upon the hypotenuse of a right triangle as diameter passes through the vertex of the right angle. 


\section{Proposition X}

184. The opposite angles of any convex quadrilateral inscribed in a circle are supplementary.

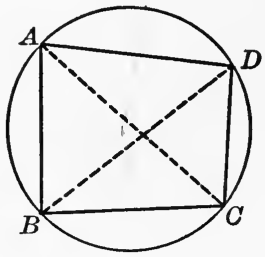

Let $A B C D$ be any convex quadrilateral inscribed in a given circle.

It is required to prove that the angles $B A D$ and $B C D$, also that the angles $A B C$ and $A D C$ are supplementary.

Proof. Join $A C$ and $B D$.

$$
\angle B A C=\angle B D C \text {; also } \angle D A C=\angle D B C \text {. Why? }
$$

Therefore the sum of $\angle B B A C$ and $D A C$ equals the sum of $\measuredangle B D C$ and $D B C$.

That is, $\angle B A D$ equals the sum of $\angle B D C$ and $D B C$.

But the sum of $\measuredangle B D C$ and $D B C$ is the supplement of $\angle B C D$. Why?

Therefore $\angle B A D$ is the supplement of $\angle B C D$.

That the angles $A B C$ and $A D C$ are supplementary may be proved in the same way, or it follows directly from the fact that the sum of the interior angles of any quadrilateral is equal to four right angles.

Conversely. If two opposite angles of a convex quadrilateral are supplementary, its four vertices lie on a circle.

Definition. Four points which lie on the same circle are said to be concyclic. 
Alternative Proof of Proposition X.

To prove that of the inscribed quadrilateral $A B C D, \measuredangle A B C$ and $A D C$ are supplementary.

$\angle A D C$ equals half of $\angle A O C, O$ being the centre of the circle.

(Prop. IX.)

$\angle A B C$ equals half of the reflex angle $A O C$.

The sum of $\measuredangle A B C$ and $A D C$ equals half of the sum of the two angles at $O$, i.e. half of four right angles.

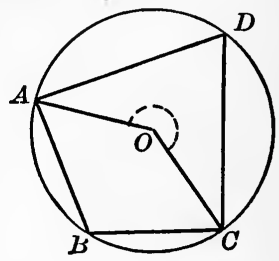

Therefore the sum of $\angle A B C$ and $A D B$ equals two right angles, or $\angle A B C$ and $A D C$ are supplementary.

\section{EXERCISES}

1. If a parallelogram is inscribed in a circle it must be a rectangle.

\section{SECTION III \\ SECANTS AND TANGENTS}

185. Definition. An unlimited straight line which intersects a circle in two points is called a secant of the circle.

186. If a secant $A B$ of any circle is rotated about one of its points of intersection $A$, the other point of intersection $B$ will move along the circle till it comes to coincide with $A$, and then if the rotation is continued will reappear sn the other side of $A$.

In that position of the secant in which the two points of intersection coincide the straight line is said to touch the circle, or to be tangent to it, and the common point of the line and circle is

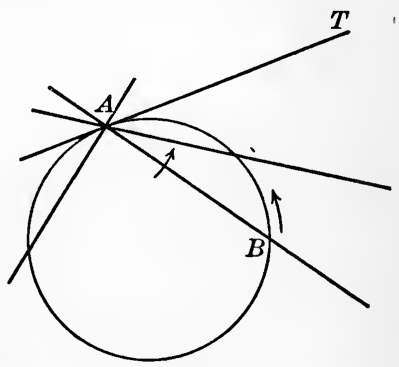
called the point of contact. The line itself is called a tangent to the circle. 
Definition. A tangent to a circle is a straight line which meets the circle, but which when produced does not cut it.

In consequence of this definition every point of a tangent to a circle lies outside the circle except the point of contact.

\section{Proposition XI}

187. The perpendicular to a diameter of a circle at one extremity is a tangent to the circle; and any other straight line through that extremity will cut the circle at a second point.

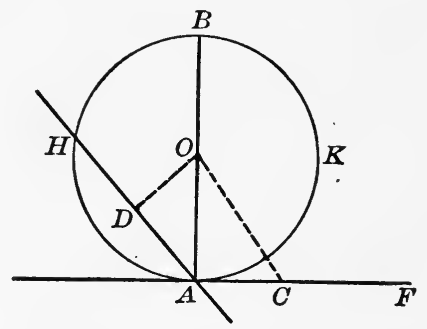

Let $A K B$ be any circle, $O$ its centre, $A B$ a diameter, $A F$ a straight line perpendicular to the diameter at the point $A$, and $A H$ any other straight line through $A$.

It is required to prove, (1), that $A F$ is a tangent to the circle, and (2), that $A H$ will meet the circle at a second point and consequently is not a tangent.

Proof. First, choose any point $C$ in $A F$, and join $O C$.

Since $A F$ is perpendicular to $A B, O C$ is greater than $O A$.

Hence $C$ lies outside the circle.

Since $A F$ meets the circle at $A$, while every other point of it lies outside the circle, it is a tangent by definition.

Next, draw $O D$ perpendicular to $A H$, meeting $A H$ at $D$.

Then $O D$ is less than $O A$, so that $D$ lies within the circle, and $A D$ produced must meet the circle a second time. (Art. 25.)

Hence $A H$ is not a tangent to the circle. 
188. Corollary I. At any point of a circle there can be drawn one and only one tangent.

For at the extremity of the diameter through the point there can be drawn one and only one perpendicular to this diameter.

189. CoRollary II. Any tangent to a circle is perpendicular to the radius drawn to the point of contact.

For, if not, it must cut the circle at a second point.

190. Corollary III. The centre of a circle lies on the perpendicular to a tangent drawn from the point of contact.

For the straight line joining the centre to the point of contact is perpendicular to the tangent, and there can be only one perpendicular to the tangent drawn from the point of contact.

191. Corollary IV. The straight line drawn from the centre of a circle perpendicular to a tangent meets it at the point of contact.

192. Conollary V. The tangent to a circle at the mid-point of any arc is parallel to the chord of the arc.

For it is perpendicular to the radius drawn to its point of contact, and the chord is also perpendicular to this radius.

(Ex. 5. p. 106.)

\section{EXERCISES}

1. Draw a tangent to a circle from a given point on the circle.

2. Draw a tangent to a circle which shall be (1) parallel to a given straight line; (2) perpendicular to a given straight line. How many such tangents can be drawn?

3. Draw a tangent to a circle which shall make a given angle with a given straight line. How many such tangents can be drawn?

4. A straight line will intersect, touch, or lie wholly outside of a given circle according as its distance from the centre is less than, equal to, or greater than a radius of the circle. 


\section{Proposition XII}

193. To draw a tangent to a circle from a given point without it.

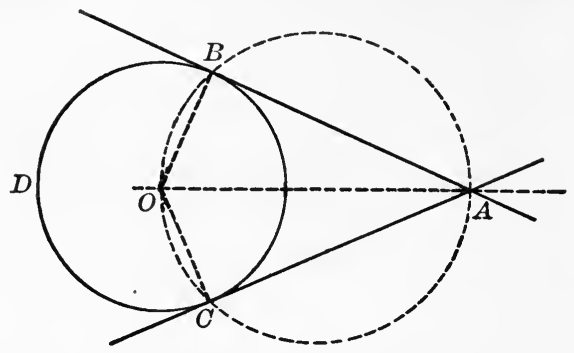

Let $B C D$ be any given circle whose centre is $O$, and let $A$ be any point without it.

It is required to draw from $A$ a straight line tangent to the given circle.

Construction. Join $A O$, and upon $A O$ as diameter describe a circle intersecting the given circle at $B$ and $C$. Join $A B$.

Then $A B$ is a tangent to the given circle.

Proof. Join $O B . \angle O B A$ is a right angle. Why?

Therefore $A B$ is tangent to the given circle at $B$. (Prop. XI.)

Similarly $A C$ is tangent to the given circle at $C$.

194. Corollary. The segments of two tangents to a circle which lie between their point of intersection and their points of contact are equal, and make equal angles with the line joining their intersection to the centre of the circle.

Suggestion. In the above diagram prove $A B$ equal to $A C$, and $\angle B A O$ equal to $\angle C A O$.

Sometimes, when no misunderstanding is likely to arise, the segment of a tangent lying between the point of contact and the point $A$ from which it is drawn will be spoken of as the tangent from $A$. In that case the above corollary may be stated as follows: 
Corollary. The two tangents to a circle drawn from any outside point are equal, and the straight line from the point to the centre of the circle bisects the angle between them.

Definition. The straight line joining the points of contact of two tangents is called their chord of contact.

195. From the above construction two tangents can be drawn to a circle from any outside point, and the question immediately arises, can more than two tangents be drawn from the same point?

Suppose in the above diagram another straight line be drawn through $A$ meeting the given circle at a point $E$, different from $B$ or $C$.

Then if $A E$ is also tangent to the circle, $\angle O E A$ is a right angle, and the circle whose diameter is $A O$ would pass through $E$ (Art. 183), and would therefore intersect the given circle at $E$.

But this is impossible since this circle already intersects the given circle at two points, $B$ and $C$.

Therefore no third tangent can be drawn through a given point.

Hence the theorem,

Two tangents and only two can be drawn to a circle from an outside point.

196. A polygon is said to be circumscribed about a circle when its sides are all tangent to the circle; and the circle is said to be inscribed in the polygon.

If a triangle is circumscribed about a circle, the bisector of any of its angles passes through the centre of the circle (Art. 194).

Hence to solve the problem:

To inscribe a circle in a given triangle,

you need only to bisect two of the angles of the triangle, and the point of intersection of these bisectors will be the centre of the required circle. For each bisector is the locus of points equidistant from two sides of the triangle, hence their point of intersection is equidistant from all three sides. 


\section{EXERCISES}

1. The angle between any two tangents to a circle is supplementary to the angle between the radii drawn to their points of contact.

2. If a quadrilateral is circumscribed about a circle, the sum of two opposite sides is equal to the sum of the other two opposite sides.

3. If a parallelogram is circumscribed about a circle it must be a rhombus and its diagonals must pass through the centre.

4. The diameter through the intersection of two tangents bisects their chord of contact at right angles.

5. Prove that the chord which joins the points of contact of two parallel tangents to a circle is a diameter.

6. If a circle is described upon one side of an isosceles triangle as diameter, show that it will pass through the mid-point of the base.

7. Construct a right triangle having given the hypotenuse and one side.

8. If $A B C$ is a triangle whose vertices lie on a circle and $A D$ bisects the angle at $A$ meeting the circle again at $D$, show that the diameter through $D$ bisects $B C$ at right angles.

9. If the opposite sides $A B$ and $C D$ of a quadrilateral $A B C D$ inscribed in a circle are produced to meet in $E$, the triangles $A E C$ and $D E B$ are equiangular, as are also the triangles $A E D$ and $C E B$.

10. Divide a circle into two arcs such that the angle contained by one shall be twice the angle contained by the other.

11. Chords of a circle whose centre is $O$ pass through a fixed point $P$; show that the locus of the mid-points of the chord is a circle whose diameter is $O P$.

12. 'Two circles intersect at the points $A$ and $B$, and a straight line $P A Q$ is drawn through $A$ cutting the circles at $P$ and $Q$, respectively. Show that if the circles are equal the chords $P B$ and $Q B$ are equal, and conversely, if $P B$ and $Q B$ are equal the circles must be equal.

13. If a circle is inscribed in a right triangle, the sum of its diameter and the hypotenuse of the triangle is equal to the sum of the other two sides. 


\section{Proposition XIII}

197. The angle formed by a tangent to a circle and $a$ chord through its point of contact equals half the angle at the centre, subtended by the intercepted arc.

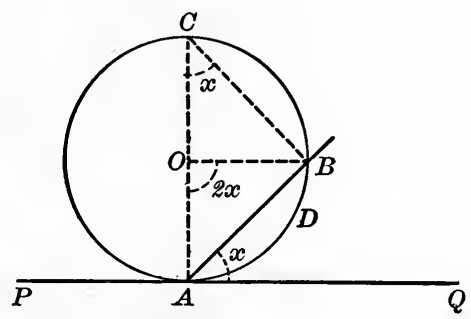

Let $O$ be the centre of the given circle, $P Q$ a tangent at $A$, and $A B$ a chord.

It is required to prove that the angle $Q A B$ equals half the angle $A O B$ at the centre, subtended by the arc $A B$.

Proof. Draw the diameter $A O$, meeting the circle a second time at $C$, and join $B O$ and $B C$.

$\angle B A Q$ is the complement of $\angle B A C$. Why?

$\angle A C B$ is also the complement of $\angle B A C$. Why?

Therefore $\angle B A Q$ equals $\angle A C B$.

But $\angle A C B$ equals half of $\angle A O B$.

Therefore $\angle B A Q$ equals half of $\angle A O B$.

(Prop. IX.)

Show in a similar manner, or otherwise, that the angle $P A B$ equals half of the reflex angle $A O B$.

198. Corollary. The angle between a tangent to a circle and a chord through its point of contact is equal to any angle inscribed in the segment on the opposite side of the chord.

By 'the intercepted are' is meant the arc falling within the angle. Thus, if we have in mind $\angle Q A B$ as the angle between the tangent and 
the chord, the intercepted arc is the minor arc $A B$; but if we think of $\angle P A B$ as the angle between the tangent and the chord, the intercepted arc is the major arc $A B$.

The corollary states that $\angle Q A B$ equals any angle inscribed in the segment $A C B$, and that $\angle P A B$ equals any angle inscribed in the segment $A D B$.

\section{Proposition XIV}

199. On a given line-segment as chord, to construct an arc of a circle which shall contain a given angle.
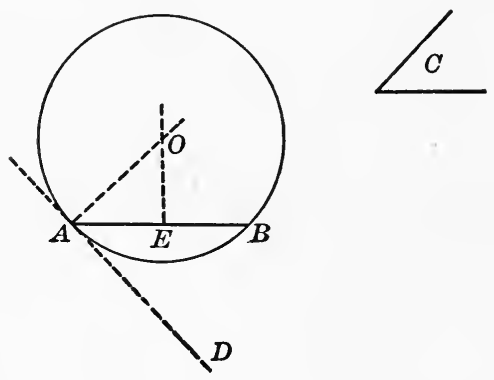

Let $A B$ be the given line-segment, and $C$ the given angle.

It is required to construct on $A B$ an arc of a circle which shall contain an angle equal to the angle $C$.

Construction. From one extremity of the given line-segment draw a straight line $A D$ making $\angle B A D$ equal to the given angle $C$.

$A B$ is to be the chord of the required arc, and if $A D$ were a tangent the given angle would be equal to the angle between a tangent and chord of the required circle.

The problem then becomes: To describe a circle tangent to $A D$ at $A$ and passing through $B$.

Find the centre of such a circle and complete the problem, giving references to all theorems employed. 


\section{Proposition XV}

200. The angle formed by two secants which intersect within a circle is equal to the sum of the two angles whose vertices are on the circle, and which are subtended by the intercepted arcs.

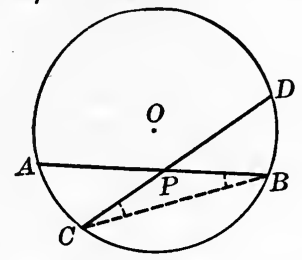

Let $A B$ and $C D$ be any two secants of a circle intersecting at $P$ within the circle.

It is required to prove that the angle $A P C$ is equal to the sum of the angles at points of the circle, subtended by the $\operatorname{arcs} A C$ and $B D$.

Proof. Join $B C$. Then $\angle A P C$ equals the sum of $\angle \mathrm{s} P B C$ and $B C P$. Why?

But $\angle P B C$, or $\angle A B C$, has its vertex on the circle and is subtended by the are $A C$.

And $\angle B C P$, or $\angle B C D$, is subtended by the arc $B D$.

Therefore, etc.

In just the same way it can be shown that the supplementary angle $A P D$ equals the sum of the angles subtended by the arcs $A D$ and $B C$.

201. If the point of intersection $P$ lies on the circle one of the intercepted arcs vanishes, but the theorem as stated for an internal point is still true. For $\angle A P C$, or its vertical angle, is subtended by the arc $B D$, while $\angle A P D$ equals the sum of the angles whose vertices are on the circle and which are subtended by the $\operatorname{arcs} B P$ and $P D$. This will readily be seen if you join $B D$.

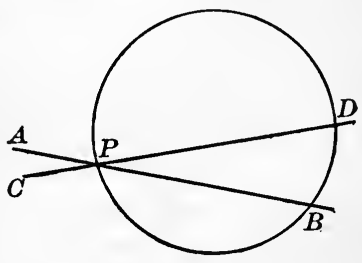




\section{Proposition XVI}

202. The angle formed by two secants which intersect without a circle is equal to the difference between the two angles whose vertices are on the circle and which are subtended by the intercepted arcs.

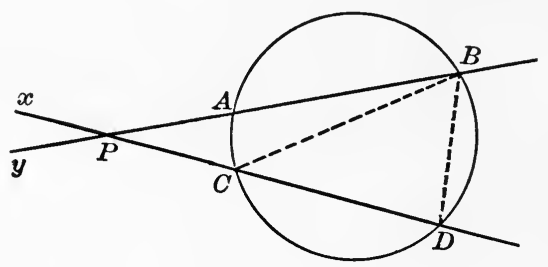

Let $A B$ and $C D$ be any two secants of a circle, intersecting at $P$, without the circle.

It is required to prove that the angle $A P C$ is equal to the difference between the angles at points of the circle, subtended by the ares $A C$ and $B D$.

Proof. Join $B C$. Then $\angle A P C$ equals the difference between $\angle \mathrm{s} A B C$ and $B C D$.

(Art. 100.)

But $\angle A B C$ is subtended by the are $A C$.

And $\angle B C D$ is subtended by the arc $B D$.

Therefore, etc.

In this case the supplementary angle $A P x$ equals the sum, not the difference, of $\angle \mathrm{s} A B D$ and $B D C$, or the sum of the angles subtended by the $\operatorname{arcs} A D$ and $B C$.

\section{EXERCISES}

1. Construct a triangle having given the base, the vertical angle, and one side. What is the limitation on the length of this side?

2. Construct a triangle having given the base, the vertical angle, and the altitude. What is the limitation upon the altitude?

3. Coustruct a triangle having given the base, the vertical angle, and the sum of the sides. 
203. Suppose one of the secants $A B$ becomes a tangent at $A$, the two intersection points $A$ and $B$ thus coinciding at $A$. Then $\angle A P C$ between the tangent and the secant equals the difference between $\angle A C D$ and $\angle C A P$. But $\angle A C D$ is subtended by the in-

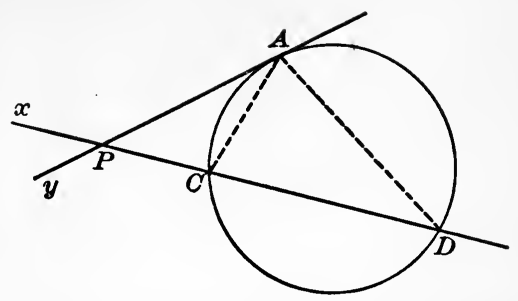
tercepted arc $A D$, or $B D$ since $A$ and $B$ coincide; and $\angle C A P$ equals an angle subtended by the intercepted arc $A C$. (Prop. XIII.)

Hence, just as in the case of two secants, we have,

THEоREм. The angle between a tangent and a secant equals the difference between the angles whose vertices are on the circle and which are subtended by the intercepted arcs.

The supplementary angle $A P x$ equals the sum of $\angle \mathrm{s} P A D$ and $P D A$.

But $\angle P A D$ equals an angle subtended by the arc $A C D$, while $\angle P D A$ is subtended by the arc $A C$.

That is, the supplementary angle $A P x$ equals the sum of the angles subtended by the ares $A D$ and $A C$ (or $B C$ ), just as in the case of the two secants.

Suppose, finally, that both secants become tangents, the intersection points $A$ and $B$ coinciding at $A$, while $C$ and $D$ coincide at $C$.

Then $\angle A P C$ equals the difference between $\angle \mathrm{s} A C z$ and $P A C$, i.e. equals the difference between the angles subtended by the $\operatorname{arcs} A K C$

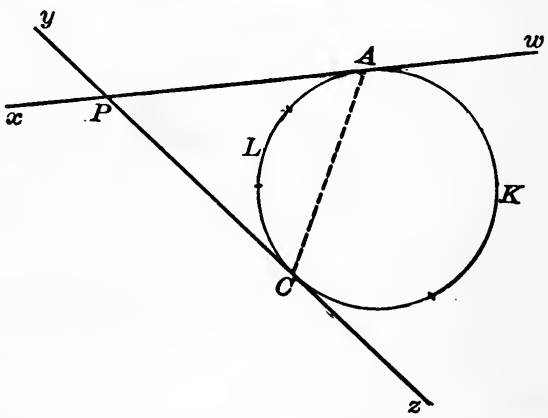
and $A L C$. And $\angle A P y$ equals the sum of $\angle \mathrm{s} P A C$ and $P C A$, i.e. equals twice the angle subtended by the arc $A L C$.

\section{Principle of Continuity}

204. The preceding discussion of the different phases of Propositions $\mathrm{XV}$ and XVI leads us to the consideration of a principle which is generally recognized in geometry as fundamental. We shall take up in the first place some very simple illustrations of the principle and then proceed 
to more complicated ones, such as are involved in the theorems just considered.

1. Suppose we have given any line-segment $A B$ marked out on an unlimited straight line $m$. If we choose a point $C$ on this line between $A$

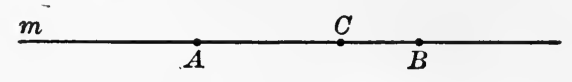

and $B$, we say that the line-segment $A B$ equals the sum of the linesegments $A C$ and $C B$.

Or, expressed algebraically,

$$
A B=A C+C B .
$$

This is true no matter where the point $C$ is chosen between $A$ and $B$, or if $C$ coincides with either $A$ or $B$, for in the latter case one of the segments becomes zero and the other equals $A B$.

If, however, $C$ is chosen on the line $m$, not between $A$ and $B$, we

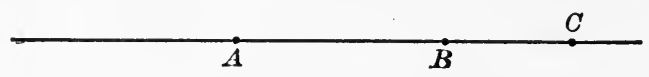

are accustomed to say that the line-segment $A B$ equals the difference between the line-segments $A C$ and $B C$,

Or, expressed algebraically,

$$
A B=A C-B C .
$$

In order to avoid the necessity of varying the statement for different positions of the point $C$, it is convenient to think of a line-segment $A B$ as having a definite sense, i.e. as though it started at $A$ and ran to $B$. The line-segment $B A$ would then run in the opposite sense.

If we attach the algebraic term positive to the line-segment $A B$, then the line-segment $B A$ should be thought of as negative, since positive and negative always indicate opposites in sense.

Thus the line-segment $A B=-$ the line-seginent $B A$, and "6 " $\quad B A=-$ " $A B$.

So for any line-segment whatever $P Q=-Q P$.

With this understanding, the expression $A C-B C$ is just the same as $A C+C B$.

Hence, if we attach to a line-segment a distinguishing sign, positive or negative, according as it is traversed in the one sense or in the other, no matter where the point $C$ may be chosen upon the unlimited straight line, whecher between $A$ and $B$ or not, it is always true that

$$
A B=A C+C B \text {. }
$$


So also, no matter how the points $M, N, P$, may be placed upon a straight line,

$$
M N+N P=M P,
$$

or expressed in words, the result of going from $M$ to $N$ and then from $N$ to $P$ is the same as going from $M$ to $P$.

So again for any four points $P, Q, R, S$, upon a straight line,

or

$$
\begin{aligned}
& P Q+Q R+R S=P S, \\
& P R+R S+S Q=P Q .
\end{aligned}
$$

Many theorems in geometry consist simply of a statement of some relation among the parts of a geometrical figure, and the principle to which we have referred affirms that this relation, once true and properly interpreted, remains true when the figure changes continuously from one form to another, subject to the conditions under which it was first described. For this reason the principle is called the principle of continuity.

Thus in the case just considered

$$
A B=A C+C B
$$

when $C$ lies on the line $m$ between $A$ and $B$. The principle of continuity affirms that this relation remains true when $C$ moves anywhere along the line $m$, if the signs of the segments be taken into account.

2. In Proposition $\mathrm{X}$ we proved that if $A Q B P$ is a convex quadrilateral inscribed in a circle, the angles $B Q A$ and $B P A$ are supplementary, and it follows immediately that $\angle B P T$ equals $\angle B Q A$.

Now suppose in the figure that the points $A, Q, B$, remain fixed while $P$ moves along the circle toward $A$, and finally coincides with $A$. During this motion $\angle B P A$ remains constant (Prop. IX, Cor. III), as does also $\angle B P T$. When $P$ comes to coincide with $A, A P$ becomes the tangent at $A$, and $B P$ takes the position $B A$ and $\angle B P T$ falls into the position of $\angle B A T$.

But

$$
\angle B P T=\angle B Q A \text {. }
$$

\section{Therefore $\angle B A T=\angle B Q A$.}

This last statement, however, is nothing else than the theorem of Proposition XIII. So, by letiing the secant $A T$ vary continuously till it takes the position of a tangent, we pass from the theorem of Proposition X to that of Proposition XIII. In other words, Proposition XIII is merely a special case of Proposition $\mathbf{X}$.

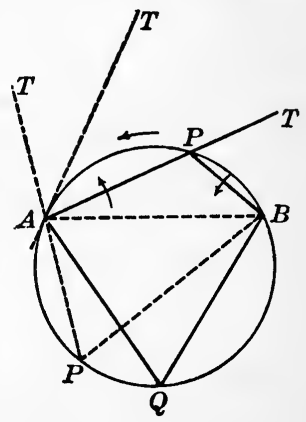


If the rotation of the secant $A T$ is continued beyond the position of a tangent, $\angle \mathrm{s} B P T$ and $B Q A$ become angles in the same segment.

During the process our figure has changed its form continuously, and the theorem with which we started has developed forms which were at first considered to be different, but which we now see to be merely different phases of the same theorem.

3. Now turn to Proposition $\mathrm{XV}$. The theorem states that $\angle B P D$ equals the sum of the angles whose vertices are on the circle and which are subtended by the arcs $B D$ and $A C$, and it was shown in connection with this proposition that $\angle A P \dot{D}$ equals the sum of the angles subtended in the same way by the ares $A D$ and $B C$.

It should be noticed that the arcs mentioned above have been named in each case, starting from the points $A$ and $B$; also that if we think of an initial and a terminal point of an arc, as we did of a linesegment, then $A C$ and $B D$

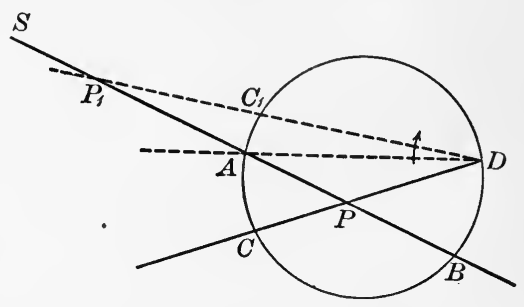
run round the circle in the same sense; so also do $A D$ and $B C$.

Suppose now we make an agreement as to the signs to be attached to arcs, similar to that which was made upon line-segments, viz., arcs which run round the circle in the same sense shall have the same sign, while arcs which run round the circle in opposite senses shall have opposite signs.

So also angles subtended by arcs of the same sign shall be of the same sign, while angles subtended by arcs of opposite signs shall be of opposite signs.

In the figure before us let us hold the points $A, B, D$, fixed and rotate the line $D C$ about $D$ in the sense indicated by the arrowhead. Then $C$ will move along the circle toward $A$ till it coincides with $A$, and will then pass to the other side. The intersection point $P$ of the two secants must move out along $B A$ till it likewise coincides with $A$ and will then go beyond.

To start with we have these two relations from Proposition XV.

$\angle D P B=$ angle subtended by $\operatorname{arc} D B+$ angle subtended by arc $C A$.

$\angle D P A=$ angle subtended by $\operatorname{arc} D A+$ angle subtended by arc $C B$.

When, by the rotation of $D C, C$ comes to coincide with $A$, the arc $C A$ vanishes, but the two relations are easily seen to remain true. 
If $C$ passes beyond $A$ into the position $C_{1}$, say, so that $P$ goes outside the circle, the $\operatorname{arcs} C_{1} A$ and $D B$ have opposite signs, and the angles subtending them must also be considered as having opposite signs. The algebraic sum on the right of the first relation then becomes the numerical difference of the angles, and the relation expressed becomes the same as that stated in Proposition XVI.

As $P$ passes outside the circle, the $\operatorname{arcs} C B$ and $D A$ overlap, but remain of the same sign. So the second relation is unaltered for Proposition XVI.

The figure is capable of still further variation which would serve to develop the other special cases given under Proposition XVI. For instance, when the point $P$ is outside, we might hold $P$ fixed, and rotate $P D$ till it becomes a tangent. The $\operatorname{arcs} A C$ and $B D$ still have opposite signs, while $A D$ and $B C$ have the same signs, etc.

Thus by making application of the principle of continuity, and by suitable conventions as to sign, Proposition XVI and the special cases which were considered under it, are shown to be simply modifications of Proposition XV. And so it frequently happens that propositions which are at first glance very different, can, by the application of the principle of continuity, be harmonized under one general statement.

\section{EXERCISES}

1. If from the point of contact of a tangent to a circle, a chord is drawn, the perpendiculars from the mid-point of either of the arcs so formed to the chord and the tangent are equal.

2. $A B C$ is a triangle inscribed in a circle, and from any point $D$ in $B C$ a straight line $D E$ is drawn parallel to $C A$, meeting the tangent at $A$ in $E$. Show that a circle may be described through the four points $A, E, B, D$.

3. Two circles intersect at $A$ and $B$, and through $A$ two straight lines $P A Q$ and $R A S$ are drawn cutting one circle in $P$ and $R$, respectively, and the other in $Q$ and $S$. Show that if the chord $P R$ is equal to the chord $Q S$, the circles must be equal; and conversely, that if the circles are equal the chords $P R$ and $Q S$ will be equal.

4. Prove that the line bisecting any interior angle of a quadrilateral inscribed in a circle and the line bisecting the opposite exterior angle intersect on the circle.

5. Prove that the straight lines which bisect the vertical angles of all triangles having the same base and equal vertical angles, have one point in common. 


\section{SECTION IV}

\section{CIRCLES IN CONTACT}

205. Definition. Two circles are said to touch each other, or to be in contact, when they have a common tangent at a common point.

This common point is called the point of contact.

\section{Proposition XVII}

206. When two circles touch each other their point of contact and their two centres lie in a straight line.

For the perpendicular to their common tangent at the point of contact must pass through the centre of each circle.

(Prop. XI. Cor. III.)

\section{Proposition XVIII}

207. Two circles which touch each other can have no other point in common than the point of contact.
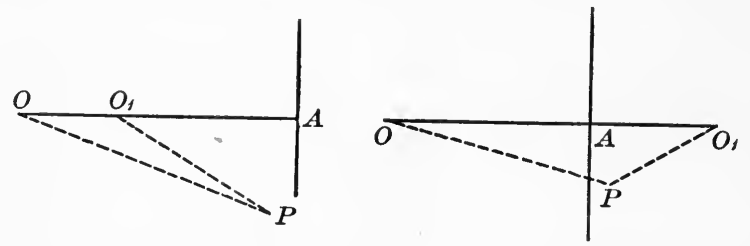

By the preceding theorem the point of contact and the two centres are collinear.

The two centres cannot coincide, for then the circles would be identical, having a common centre and a common point.

Two cases must then be considered, according as the centres lie (1) on the same side, (2) on opposite sides of the point of contact.

Let $O$ and $O_{1}$ be the centres of the two circles, and let $A$ be their point of contact. 
If possible, let $P$ be another point common to the two circles. Then $O A=O P$, and $O_{1} A=O_{1} P$. Hence in either of the two possible cases, one side of the triangle $O P O_{1}$ must equal the sum of the other two sides, which is impossible.

Therefore, two circles tangent at $A$ can have no other common point.

When the two centres lie on the same side of the common tangent, the circles are said to have internal contact, or to touch each other internally; when the
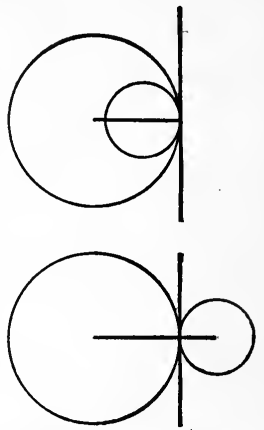
centres lie on opposite sides the circles have external contact, or touch each other externally:

In the first case one circle lies inside of the other, in the second case each lies outside of the other.

208. CoRolla RY. If two circles have a common point on their line of centres, they must have a common tangent at that point.

For the tangent to either circle at that point is perpendicular to the line of centres and the two tangents must therefore coincide. That is the two circles have a common tangent at that point.

\section{EXERCISES}

1. Two circles touch each other externally or internally at a point, and through that point a straight line is drawn to cut the two circles. If the points of intersection be joined to the respective centres, the two straight lines so drawn will be parallel.

2. If the distance between the centres of two circles is equal to the sum of their radii, the two circles must touch each other externally.

3. If the distance between the centres of two circles is equal to the difference of their radii, the two circles must touch each other internally.

4. Describe a circle passing through a given point and touching a given circle at a given point. 


\section{Proposition XIX}

209. To draw a common tangent to two given circles, each of which lies wholly outside of the other.

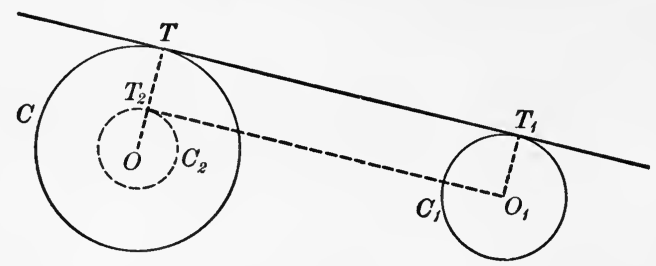

Let $C$ and $C_{1}$ be the given circles, $O$ and $O_{1}$ their centres, and suppose the radius of $C$ greater than the radius of $C_{1}$.

It is required to draw a straight line which shall touch both of these circles.

Construction. With centre $O$ and radius equal to the difference between the radii of $C$ and $C_{1}$ describe a circle $C_{2}$.

From $O_{1}$ draw a tangent to $C_{2}$, the point of contact being $T_{2}$.

[Notice that two such tangents can be drawn.]

Join $O T_{2}$ and produce to meet $C$ at $T$.

From $O_{1}$ draw a radius of $C_{1}$, viz. $O_{1} T_{1}$, parallel to $O T$, and on the same side of the line of centres.

Draw the straight line $T T_{1}$, which shall be the required common tangent.

Proof. The quadrilateral $T T_{1} O_{1} T_{2}$ is a parallelogram. Show why.

The angle $O_{1} T_{2} T$ is a right angle. Why?

Therefore the straight line $T T_{1}$ is perpendicular to a radius of each of the given circles at points $T$ and $T_{1}$ of the circles.

It is therefore tangent to both circles.

\section{EXERCISES}

1. Show that by drawing the other tangent from $O_{1}$ to the circle $C_{2}$, a second common tangent to the two given circles could be found. 
2. Show that by drawing a circle $C_{3}$ with centre $O_{1}$ instead of $O$, and with radius equal to the sum of the radii of the given circles, and also by drawing the parallel radii on opposite sides of the line of centres, two other common tangents to the given circles could be found.

The two common tangents first drawn are called the direct common tangents, the other two the transverse common tangents.

3. Show that when the two given circles are equal the direct common tangents are parallel. How does the construction differ in this case ?

4. Show that when the two given circles are in contact, the centre $O$ lies upon the circle $C_{3}$ (as drawn to obtain the transverse tangents); and that when the given circles intersect, the centre $O$ lies within the circle $C_{3}$.

Hence, when the two given circles are in contact, the two transverse common tangents coincide and become the common tangent at the point of contact ; and when the two given circles intersect, the transverse common tangents cannot be drawn.

5. If in any two given circles which are in contact there be drawn two parallel diameters, the point of contact and an extremity of each diameter lie in the same straight line.

Suggestion. Draw the line of centres, and join an extremity of each diameter to the point of contact. Show that the two lines so drawn make equal angles with the line of centres.

6. If two circles have a common point not on the line of centres, the circles must intersect at that point, and also at another point which is situated symmetrically with it relative to the line of centres.

Suggestion. The foot of the perpendicular from the common point to the line of centres lies inside of both circles; hence the circles must intersect.

7. Two circles touch each other internally or externally at the point $A$, and through $A$ two straight lines are drawn cutting one circle in $P$ and $R$, respectively, and the other circle in $A$ and $S$. Show that $P R$ is parallel to $Q S$.

8. Two circles touch one another at the point $A$, and have a common tangent meeting them at the points $B$ and $C$, respectively. Show that the circle whose diameter is $B C$ passes through $A$. Show also that if the lines $B A$ and $C A$ are produced to cut the circles again at $C^{\prime}$ and $B^{\prime}$, respectively, the lines $B B^{\prime}$ and $C C^{\prime}$ will be diameters.

Suggestion. If the common tangent at $A$ intersects the common tangent $B C$ at $M, M A=M B=M C$ (Art. 194). Therefore $\angle B A C$ is a right angle. 


\section{MISCELLANEOUS EXERCISES}

1. All chords of the greater of two given concentric circles which are tangent to the smaller are equal.

2. What is the locus of the centres of circles touching two given straight lines which intersect?

3. Describe a circle of given radius which shall touch two given intersecting straight lines. Show that there are four such circles.

4. A circle is described on the radius of another as diameter. Prove that any chord of the greater circle drawn through their point of contact is bisected by the lesser circle.

5. Through a point of intersection of two given circles draw the greatest possible line-segment which is terminated both ways by the circles.

6. If $A B$ and $C D$ are two equal chords in a circle, prove that of the two pairs of straight lines $A D, B C$, and $A C, B D$, one pair are equal and the other parallel.

7. Given two circles and a tangent to each, these being parallel; if the points of contact of the tangents be joined by a straight line, the tangents at the points where this straight line cuts the circles a second time are also parallel.

8. Two radii of a circle are at right angles and when produced are cut by a straight line which touches the circle. Show that the other tangents drawn from the points of intersection with the radii are parallel.

9. If two circles touch each other and a chord be drawn through the point of contact, the tangents at the other points where the chord meets the circles are parallel.

10. From all the points of a circle equal and parallel line-segments are drawn in the same direction. What is the locus of their extremities?

11. From any point within a circle straight lines are drawn to the circle; show that the locus of their mid-points is a circle. What would be the locus of the mid-points if the lines were drawn from a point on or outside the circle?

12. Let $A B$ be any diameter of a circle whose centre is $O$, and let $C$ be any point on this diameter produced. Through $C$ draw any secant cutting the circle at $D$ and $E$. If the exterior part $C D$ of this secant is equal to a radius of the circle, show that the angle $E O A$ is three times the angle $D O B$. 
13. What is the locus of the mid-points of equal chords of a circle ?

14. If two equal chords of a circle are produced to meet outside the circle, prove that the exterior parts are equal. What is the corresponding property when the chords intersect inside the circle? Notice the principle of continuity.

15. What is the locus of the centres of circles of constant radius which touch a given circle?

16. A straight line is drawn intersecting two concentric circles. Show that the line-segments intercepted between the circles are equal.

17. Circles are described on the sides of a quadrilateral as diameters. Show that the common chord of the circles described on two adjacent sides is parallel to the common chord of the other two circles.

18. If $A B$ and $A^{\prime} B^{\prime}$ are two equal line-segments lying in a plane, but not parallel, find a point $O$ such that if the line $A O$ be rotated about it through a certain angle, $A$ will coincide with $A^{\prime}$ and at the same time $B$ with $B^{\prime}$.

19. Three circles touch one another externally (each touching the other two), at the points $A, B, C$; the straight lines $A B, A C$, are produced to meet the circle $B C$ at $D$ and $E$. Show that $D E$ is a diameter of this circle parallel to the line of centres of the other two circles.

20. If $A B$ is a fixed diameter, and $D E$ an arc of constant length in a given circle, and the lines $A E, B D$ intersect at $P$, show that the angle $A P B$ is constant.

21. Three concurrent straight lines make fixed angles with each other. If they be moved so that two of them constantly pass through fixed points, the third must also pass through a fixed point.

22. A triangle is inscribed in a circle. Show that the sum of the angles contained in the three arcs subtended by the sides is equal to four right angles.

23. If $A B C$ is any triangle and a circle is described through the vertices $B$ and $C$, cutting the sides $B A$ and $C A$, at the points $P$ and $Q$, prove that $P Q$ is parallel to a fixed straight line.

24. If $E$ is a point on one of the diagonals $A C$ of a parallelogram $A B C D$, and circles are described about $D E A$ and $B E C$, show that the other point of intersection of these two circles must lie on $B D$.

25. If through $P$, any point on one of two circles which intersect at $A$ and $B$, the straight lines $P A$ and $P B$ are drawn to meet the other circle at $Q$ and $R$, prove that the arc $Q R$ is of constant length, or that the length of $Q R$ is independent of the position of the point $P$. 
1. Definitions.

\section{SUMMARY OF CHAPTER II}

(1) Circle, centre, radius, diameter. $§ 147$. See also p. 20.

(2) Arc of a Circle - any portion of a circle terminated by two points. $\$ 153$.

(3) Chord of a Circle - the straight line joining two points of a circle. $\S 153$.

(4) Conjugate Arcs of a Circle - two arcs which together make up the whole circle. $\$ 153$.

(5) Semicircle - the arc of a circle subtended by a diameter. $§ 154$.

(6) Segment of a Circle - the figure formed by an arc and its subtending chord. $§ 156$.

(7) Sector of a Circle - the figure formed by an arc and the two radii to its extremities. $\S 156$.

(8) Inscribed Figure - a rectilinear figure is said to be inscribed in a circle when its vertices lie on the circle. $\$ 177$.

(9) Circumscribed Figure - a rectilinear figure is said to be circumscribed about a circle when its sides are all tangent to the circle. $\S 196$.

(10) Concyclic Points - points which lie on the same circle. §184.

(11) Secant of a Circle - an unlimited straight line which intersects a circle in two points. $\S 185$.

(12) Tangent to a Circle - a straight line which meets a circle, but which when produced does not cut it. $\$ 186$.

(13) Chord of Contact - the straight line joining the points of contact of two tangents. $\S 194$.

(14) Circles in Contact - two circles which have a common tangent at a common point. $\S 205$.

(15) Direct and Transverse Common Tangents. See Ex. 2, p. 133.

(16) Principle of Continuity - the principle which asserts that a relation among the parts of a geometrical figure, once true and properly interpreted, remains true when the figure changes continuously from one form to another, subject to the conditions under which it was first described. $\$ 204$.

2. Problems.

(1) To find the centre of a circle which passes through three given points, or to circumscribe a circle about a given triangle. $§ 151$.

(2) To inscribe a circle in a given triangle. $\$ 196$.

(3) To draw a tangent to a circle from a given point on it. $\S 187$. 
(4) To draw a tangent to a circle from a given point without it. $\S 193$.

(5) On a given line-segment to construct an arc of a circle which shall contain a given angle. $\S 199$.

(6) To draw a common tangent to two given circles, each of which lies wholly outside of the other. $§ 209$.

3. Theorems on the Coincidence of Circles.

(1) Two circles in a plane which have the same centre and equal radii coincide throughout. $\S 148$.

(2) Two circles in a plane which have the same centre and one point in common coincide throughout. $\$ 148$.

(3) Two circles which have equal radii can be made to coincide, and hence are identically equal ; and, conversely, equal circles have equal radii. $\S 148$.

(4) Through three points not in the same straight line, one and only one circle can be described. $\S 151$.

(5) Two circles which coincide at three points coincide throughout. $\S 151$.

(6) Two different circles can have at most two points in common. $\S 151$.

(7) A straight line can intersect a circle in at most two points. $\$ 152$.

4. Theorems on Arcs, their Chords and Angles.

(1) In equal circles or in the same circle:

(a) Equal angles at the centre are subtended by equal arcs; and of two unequal angles the greater is subtended by the greater arc. $\$ \S 157,158$.

(b) Equal arcs subtend equal angles at the centre; and of two unequal arcs the greater subtends the greater angle at the centre. $\$ \S 159,160$.

(c) Equal arcs are subtended by equal chords; and of two unequal minor arcs, the greater is subtended by the greater chord. $\S \S 161,162$.

(d) Equal chords subtend equal arcs; and of two unequal chords, the greater subtends the greater arc. $§ 163$.

(e) Equal chords subtend equal angles at the centre, and of two unequal chords, the greater subtends the greater angle. $\S 164$.

(2) An angle whose vertex is on a circle equals half the angle at the centre subtended by the same arc. $\S 178$.

(3) The angle in a semicircle is a right angle. $\$ 179$. 
(4) An angle in an arc greater than a semicircle is acute, and an angle in an arc less than a semicircle is obtuse. $\S 180$.

(5) All angles in a circle subtended by the same arc, or by equal arcs, are equal. $\S 181$.

(6) Equal angles on the same base, and on the same side of it, have their vertices on an arc of a circle of which the given base is the chord. $\S 182$.

5. Theorems on Chords in relation to the Centre.

(1) The perpendicular bisector of any chord of a circle passes through the centre. $\S 155$.

(2) The line drawn from the centre perpendicular to a chord bisects the chord, and, if produced, bisects the arc subtended by the chord. $\S 165$.

(3) The straight line drawn from the centre to the mid-point of a chord is perpendicular to the chord. $\S 166$.

(4) The mid-points of a system of parallel chords all lie on a diameter perpendicular to the chords. $\S 167$.

(5) The diameter which bisects a chord also bisects the angle at the centre subtended by the chord. $\S 169$.

(6) In the same circle or in equal circles :

(a) Equal chords are equidistant from the centre; and of two unequal chords, the greater is nearer to the centre than the less. $\$ 170$. And, conversely -

(b) Chords equidistant from the centre are equal; and of two chords unequally distant, the one nearer the centre is the greater. $\S 171$.

(7) A diameter is the greatest chord that can be drawn in any circle. $\S 172$.

6. Theorems relating to Tangents.

(1) The perpendicular to a diameter of a circle at one extremity is a tangent to the circle; and any other straight line through that extremity will cut this circle at a second point. $\$ 187$.

(2) Any tangent to a circle is perpendicular to the radius drawn to the point of contact. $\S 189$.

(3) The centre of a circle lies on the perpendicular to a tangent drawn from the point of contact. $\S 190$.

(4) The straight line drawn from the centre of a circle perpendicular to a tangent meets it at the point of contact. $\$ 191$.

(5) The tangent to a circle at the mid-point of any arc is parallel to the chord of the arc. $\S 192$. 
(6) At any point of a circle there can be drawn one and only one tangent. $\$ 188$.

(7) From a point outside of a circle there can be drawn two and only two tangents. $\S 195$.

(8) The two tangents to a circle drawn from any outside point are equal, and the straight line from the point to the centre of the circle bisects the angle between them. $\S 194$.

(9) The angle formed by a tangent to a circle and a chord through its point of contact equals half the angle at the centre, subtended by the intercepted arc; or is equal to the angle in the segment on the opposite side of the chord. $\$ \S 197,198$.

\section{Theorems on Two Circles.}

(1) The straight line joining the centres of two intersecting circles bisects their common chord at right angles. $\$ 168$.

(2) When two circles touch each other, their point of contact and their two centres are collinear. § 206.

(3) Two circles which touch each other can have no other point in common than the point of contact. $\$ 207$.

(4) When two circles have a common point on their line of centres, they must have a common tangent at that point. $\$ 208$.

\section{Miscellaneous Theorems.}

(1) Of all straight lines which can be drawn to a circle from a point within it, not the centre, the greatest is that which passes through the centre, and the least is that which, if produced backward, would pass through the centre; and of any two others, the greater is that which makes the less angle with the diameter through the point. $\S 173$.

(2) From any point within a circle two equal straight lines can be drawn to the circle; these make equal angles with the diameter through the point. $\$ 174$.

(3) The circle described upon the hypotenuse of a right triangle as diameter passes through the vertex of the right angle. $\S 183$.

(4) The opposite angles of any convex quadrilateral inscribed in a circle are supplementary. $\S 184$.

Conversely, if two opposite angles of a convex quadrilateral are supplementary, its vertices are concyclic. $\$ 184$.

(5) The angle formed by two secants of a circle is equal to the sum or the difference of the angles whose vertices are on the circle and which are subtended by the intercepted arcs, according as the secants intersect within or without the circle. $\$ \S 200,202$. 


\section{CHAPTER III}

\section{SIMILAR RECTILINEAR FIGURES}

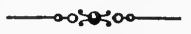

\section{SECTION I}

\section{MEASUREMENT, RATIO, AND PROPORTION}

\section{On Measurement}

210. If you are asked to measure a stick or a piece of cloth, you first select a magnitude which is well known, say a footrule or a yard-stick, and then compare the length of the given stick or piece of cloth with the chosen unit length. You find perhaps that the given stick is four feet long, or that the piece of cloth is five yards long.

What is meant by saying that the stick is four feet long? Simply that the length of the stick is four times the length of the foot-rule.

211. Definition. To measure anything is to find out by experiment how many times it will contain a chosen unit.

If one magnitude contains another an integral number of times, the first is called a multiple of the second, and the second a measure of the first.

The measure of any magnitude is a number expressing how many times it contains the unit of measure.

212. The unit of measure and the thing to be measured must of course be magnitudes of the same kind.

In the case mentioned we had two magnitudes of the same 
kind, viz. the length of the stick and the length of the footrule, and we say that the measure of the former is four when the latter is taken for unit.

Exercise. What is the measure of 12 feet when a yard is taken for unit? Of a dollar when a dime is taken for unit? Of a mile when a foot is taken for unit? Of 68 when 4 is taken for unit?

213. If two magnitudes are multiples of the same magnitude, or, in other words, if two magnitudes have a common measure, they are said to be commensurable.

The numbers 21 and 33 have a common measure 3 ; the numbers 17 and 43 have a common measure 1 ; the numbers $4 \frac{1}{2}$ and $3 \frac{1}{6}$ have a common measure $\frac{1}{6}$ or $\frac{1}{12}$.

Two magnitudes which have no common measure are called incommensurable.

As examples of incommensurable magnitudes the following may be mentioned :

(1) The circumference and diameter of a circle; (2) the side and diagonal of a square.

The numbers 7 and $\sqrt{11}, 1$ and $\sqrt{2}$ are incommensurable.

214. The standard unit for measuring lengths in the English or common system is the yard, and in the French system, which is coming to be used almost universally for scientific purposes, the standard unit is the metre.

These units are defined to be lengths equal to the lengths of certain pieces of metal which are carefully preserved, the first in London, the second in Paris.

For making small measurements these units are too large for convenient use, and so fractional parts of them are used in such cases ; for example, 'a foot' is $\frac{1}{3}$ of a yard; 'an inch' is $\frac{1}{12}$ of a foot or $\frac{1}{36}$ of a yard; 'a centimetre' is $\frac{1}{100}$ of a metre, and so on.

On the other hand, for making large measurements we use 'a mile,' which equals 1760 yards, and so on. 
215. For measuring angles the standard unit is the right angle, defined in Article 15, page 9 .

This unit again is too large for convenient use, and a smaller unit is commonly employed in making actual measurements, namely, a degree.

A degree is defined to be $\frac{1}{90}$ of a right angle.

A minute is $\frac{1}{60}$ of a degree, and a second, $\frac{1}{60}$ of a minute.

\section{EXERCISES}

1. How many degrees in two right angles? In two-thirds of a right angle? In one-fifth of a right angle?

2. How many degrees in the sum of the angles of a triangle?

3. How many degrees in each of the angles of an equilateral triangle ?

4. How many degrees in each of the angles of an isosceles right triangle?

5. One angle of a right triangle equals $30^{\circ}$. How many degrees in each of the others?

6. The vertical angle of an isosceles triangle equals $18^{\circ}$. How many degrees in each of the base angles?

7. One of the base angles of an isosceles triangle equals $50^{\circ}$. How large is the vertical angle?

8. One angle of a parallelogram equals $45^{\circ}$. What is the measure of each of the other angles?

\section{ON Ratio}

216. When we speak of the ratio of one quantity to another, we have in mind their relative magnitude. By this we mean not how much the one is greater or less than the other, but how many times the one is as great as the other.

A ratio can be expressed only between two quantities of the same kind.

When dealing with numbers we say that the ratio of one to another is the quotient arising from dividing the first by the 
second, since the quotient expresses how many times the one is as great as the other.

Thus the ratio of 8 to 4 is 2 , the ratio of 7 to 3 is $2 \frac{1}{3}$, the ratio of 5 to 9 is $\frac{5}{9}$, etc. ; but the division of one geometrical magnitude by another has no meaning unless the first is an exact multiple of the second, or until a meaning is assigned by definition. Consequently, the numerical value of the ratio of two such magnitudes must sometimes be got at in a roundabout way.

217. The same two quantities $A$ and $B$ have two different ratios, viz. the magnitude of $A$ as compared with $B$, and the magnitude of $B$ as compared with $A$.

The first is expressed $A: B$, and should be read 'the ratio of $A$ to $B$ '; the second is expressed $B: A$ and should be read ' the ratio of $B$ to $A$.'

In any ratio the first term is called the antecedent, the second term, the consequent.

218. The following postulates will serve to add definiteness to the meaning of the term 'ratio.'

Postulate 6. If $P$ and $Q$ are any two equal magnitudes and $R$ is a third magnitude of the same kind, then the ratio of $P$ to $R$ is equal to the ratio of $Q$ to $R$, i.e. if $P=Q$, then $P: R=Q: R$; and, conversely, if $P$ and $Q$ are such that $P: R=Q: R$, then $P=Q$.

Postulate 7. If $P$ and $Q$ are two unequal magnitudes, and $R$ is a third magnitude of the same kind, then the ratio of $P$ to $R$ is greater or less than the ratio of $Q$ to $R$, according as $P$ is greater or less than $Q$.

219. In order to show how the ratio of one geometrical magnitude to another can be expressed numerically, it is necessary to consider two distinct cases. 
(a) When the two given magnitudes have a common measure, or are commensurable.

(b) When the two given magnitudes have no common measure, or are incommensurable.

\section{(a) Ratio of commensurable Magnitudes}

220. Definition. The ratio of two commensurable magnitudes is the ratio of their numerical measures by a common unit.

Let $A$ and $B$ be any two magnitudes of the same kind which have a common measure $S$, and let $S$ be contained in $A m$ times, and in $B n$ times, so that $m$ is the measure of $A$, and $n$ the measure of $B$, by the common unit $S$.

Then by definition

$$
A: B=m: n \text {. }
$$

But the ratio of the number $m$ to the number $n$ is the - quotient $\frac{m}{n}$.

Therefore

$$
A: B=\frac{m}{n}
$$

221. If instead of $A$ and $B$ we take any equimultiples of these magnitudes, say $p A$ and $p B$, their measures by the common unit $S$ would be $p m$ and $p n$.

Then by definition

$$
\begin{aligned}
p A: p B & =p m: p n \\
& =\frac{p m}{p n}=\frac{m}{n} .
\end{aligned}
$$

But $\frac{m}{n}$ is the ratio of $A$ to $B$.

Therefore, the ratio of any two commensurable magnitudes is equal to the ratio of any equimultiples of those magnitudes, taken in the same order.

The ratio of $5 \mathrm{ft}$. to $7 \mathrm{ft}$. is the same as the ratio of $10 \mathrm{ft}$. to 14 $\mathrm{ft}$, , or of $25 \mathrm{ft}$. to $35 \mathrm{ft}$. 
222. Again, if instead of the common measure $S$ we use a different common measure, say $\frac{S}{t}$, so that the measure of $A$ becomes $t m$ and the measure of $B$ becomes $t n$, then

$$
\begin{aligned}
A: B & =t m: t n \\
& =\frac{t m}{t n}=\frac{m}{n} .
\end{aligned}
$$

That is, the ratio of $A$ to $B$ is not altered by a change in the unit of measure.

The ratio of $5 \mathrm{ft}$. to $7 \mathrm{ft}$. is not altered if those lengths are expressed in inches, or in yards, instead of feet.

223. For convenience we frequently write $A: B$ in the form $\frac{A}{B}$ even when $A$ and $B$ represent geometrical magnitudes; but the symbol so used should always be read as 'the ratio of $A$ to $B$,' and should not be confounded with an ordinary fraction, or symbol of division.

Thus, $\frac{\angle A B C}{\angle P Q R}$ expresses the ratio of $\angle A B C$ to $\angle P Q R$, and may be written in that form or in the usual ratio form

$$
\angle A B C: \angle P Q R \text {. }
$$

If $\angle A O B$ is at the centre of a circle subtended by the arc $A B$, and $\angle A C B$ is at a point of the circle subtended by the same arc, then $\frac{\angle A O B}{\angle A C B}=\frac{2}{1}$ and $\frac{\angle A C B}{\angle A O B}=\frac{1}{2}$, the angle $A C B$ being taken for unit of measure in each case.

(Art. 178.)

224. Whenever the first of the two given magnitudes is an exact multiple of the second, the second may be taken as the common unit of measure; and then the ratio of the first to the second is equal to the measure of the first by the second.

The ratio of 8 . to 4 is 2 , of 20 to 5 is 4 , of the diameter to a radius of a circle is 2 , of the perimeter to a side of an equilateral triangle is 3 , etc. 


\section{(b) ratio of incommensurable magnitudes}

225. Next suppose that $A$ and $B$ are two magnitudes such that no unit however small will measure them both integrally, that is, suppose that $A$ and $B$ are incominensurable.

In that case the ratio of $A$ to $B$ cannot be expressed either as a whole number (Art. 224) or as a fraction whose numerator and denominator are both whole numbers (Art. 220), since they have no common measure.

But while the ratio of two such magnitudes does not absolutely equal any integer or common fraction, it is always possible to find a common numerical fraction which will differ in value from their ratio by less than any assigned quantity however small, as we shall now proceed to show.

226. It is necessary before going further to introduce a new idea, which we shall do by means of an illustration.

A boy is to walk from $P$ to $Q$, a distance of two miles. He goes half the distance the first hour, half the remaining distance the second hour,

half the remaining distance the third hour,

and so on. Would he ever absolutely reach his distination? Could you fix a point between $P$ and $Q$, as near as you like to $Q$, beyond which he would not pass in time? Would he ever get beyond $Q$ ? .

In the first hour the boy would go 1 mile, in the second, $\frac{1}{2}$ mile; in the third, $\frac{1}{4}$ mile; in the fourth, $\frac{1}{8}$ mile; and so on.

Take these numbers $1, \frac{1}{2}, \frac{1}{4}, \frac{1}{8}, \frac{1}{16}, \frac{1}{32}, \cdots$, and let $S$ represent the sum of $n$ of them.

Then when $n=1, S=1$;

$$
\begin{aligned}
& \text { when } n=2, S=1+\frac{1}{2}=\frac{3}{2} ; \\
& \text { when } n=3, S=1+\frac{1}{2}+\frac{1}{4}=\frac{7}{4} ; \\
& \text { when } n=4, S=1+\frac{1}{2}+\frac{1}{4}+\frac{1}{8}=\frac{15}{8} ;
\end{aligned}
$$

and so on. 
Here $S$ is a variable quantity dependent upon the value of the integer $n$. As $n$ increases, $S$ increases. By taking $n$ large enough, we can make $S$ as nearly equal to the fixed number two as we please, but it can never be absolutely equal to two, and can never exceed two.

The fixed quantity two is called the limit of the variable quantity $S$.

227. Definition. If a quantity is made to vary, by changing in some definite way another quantity on which it depends, and its value approaches a fixed quantity nearer than for any assignable difference, though it cannot be made absolutely identical with it, this fixed quantity is called the limit of the variable quantity.

In the illustration of Article 226, the variable quantity $S$ is called the dependent variable, and the quantity $n$ on which it depends the independent variable.

228. Now let $A$ and $B$ be two incommensurable magnitudes, for example, two line-segments. Then $A: B$ is a so-called incommensurable ratio. Take a unit length $x$, which will measure $B$ integrally $n$ times, and apply it as often as possible to $A$, say

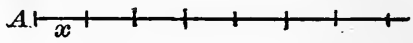
$m$ times, $m$ and $n$ being thus both integers. Since $A$ and $B$ are incommensurable, the unit $x$ will not measure $A$ exactly, and there must be a remainder which is less than $x$.

If we repeat the process, taking for unit a measure of $B$ smaller than $x$, then $m$ and $n$ will ordinarily be greater than before, and the remainder for $A$ will again be smaller than the chosen unit. By taking the unit small enough, we can make this remainder as small as we please, though it can never be made to disappear altogether.

When the chosen unit of measure is $x, A$ is greater than $m x$, but less than $(m+1) x$, while $B$ equals $n x$. 
Therefore $A: B$ is greater than $m x: n x$, but less than $(m+1) x: n x$, (Art. 218, Post. 7.) or $A: B$ is greater than $m: n$, but less than $(m+1): n$. That is, $A: B$ lies between $\frac{m}{n}$ and $\frac{m+1}{n}$. Now $\frac{m}{n}$ differs from $\frac{m+1}{n}$ by $\frac{1}{n}$. Therefore $A: B$ differs from $\frac{m}{n}$ by less than $\frac{1}{n}$.

By taking the unit of measure $x$ small enough, $n$ can be made as great as we please, i.e. $\frac{1}{n}$ as small as we please, and, consequently, smaller than any number you may choose to name.

Therefore by choosing $x$ small enough, we can always find a fractional number $\frac{m}{n}$ (whose numerator and denominator are both integers) which differs in value from the incommensurable ratio $A: B$ by less than any number you choose to name, no matter how small, i.e. by less than any assigned quantity.

The variable fraction $\frac{m}{n}$ approaches a limit, and this limit is taken as the numerical value of the ratio of $A$ to $B$.

229. Definition. The ratio of two incommensurable magnitudes $A$ and $B$ is the limit which the fraction $\frac{m}{n}$ (defined in Article 228) approaches as the unit $x$ upon which it depends is indefinitely diminished.

If $A$ and $B$ are commensurable as in Article 220, some one of the fractions found in proceeding to the limit will itself be their ratio.

230. General Theorem on Limits. If there are two variable quantities dependent on the same quantity in such a way that 
they remain always equal while each approaches a limit, then their limits are equal.

For, if the limits are not equal, they must differ by some finite quantity, say $k$; and since each variable approaches indefinitely near to its own limit, the limits differing by $k$, the two variables must come finally to differ by a finite quantity, which is contrary to the assumption that they remain always equal. Therefore the limits cannot differ by any finite quantity; in other words, the limits are equal.

231. By the definition given in Art. 220, the ratio of two commensurable magnitudes can always be expressed as the ratio between two fixed integral numbers (viz. their measures by a common unit); and by the definition of Article 229, the ratio of two incommensurable magnitudes is the limit of the ratio of two variable integral numbers.

Hence the properties of the ratios of integral numbers can be applied directly to geometrical magnitudes.

\section{Proportion}

232. Definition. If four quantities are such that the ratio of the first to the second equals the ratio of the third to the fourth, these four quantities are said to form a proportion.

That is, if the four quantities, $a, b, c, d$, are such that $a: b=$ $c: d$, then they form a proportion, or are in proportion; or, the terms $a$ and $b$ are proportional to $c$ and $d$.

A proportion may be written in the form $a: b=c: d$, or in the fractional form $\frac{a}{b}=\frac{c}{d}$, and should be read 'the ratio of $a$ to $b$ equals the ratio of $c$ to $d$,' or ' $a$ is to $b$ as $c$ is to $d$.'

Sometimes, in expressing the equality of two ratios, the double colon (::) is used instead of the ordinary sign of equality. Thus $a: b:: c: d$ means the same as $a: b=c: d$.

In a proportion the first and fourth terms are called the extremes, the second and third, the means. 
233. Theorem. If four numbers are in proportion, the product of the extremes equals the product of the means.

For if $a: b=c: d$, then $\frac{a}{b}=\frac{c}{d}$, and multiplying both sides of this equation by $b d$, we obtain $a d=b c$.

Converseiy. If the product of two numbers equals the product of two others, one pair can be the extremes and the other pair the means in a proportion.

$$
\text { If } a d=b c \text {, dividing by } b d \text { gives } \frac{a}{b}=\frac{c}{d} \text { or } a: b=c: d \text {. }
$$

234. The property expressed in the above theorem, namely, that the product of the extremes equals the product of the means, may be called the essential property of a proportion, and the terms of the proportion may be written in any order which will preserve this property.
Thus, if
$a: b=c: d$,

then $a d=b c$, and the proportion may be written either in the form

(2), $\quad b: a=d: c$,

or

$a: c=b: d$,

since these forms (2) and (3) have the same essential property as form (1).

Form (2) may be deduced from the essential property by dividing both sides by $a c$ after interchanging the sides.

Thus

$$
\begin{aligned}
& b c=a d, \\
& \frac{b c}{a c}=\frac{a d}{a c} .
\end{aligned}
$$

Therefore

$$
\frac{b}{a}=\frac{d}{c}, \text { or } b: a=d: c .
$$

And form (3) may be deduced by dividing the essential property by $c d$. 
Thus

$$
\begin{aligned}
& a d=b c, \\
& \frac{a d}{c d}=\frac{b c}{c d} .
\end{aligned}
$$

Therefore

$$
\frac{a}{c}=\frac{b}{d}, \text { or } a: c=b: d \text {. }
$$

If the two sides of the proportions (1), (2), and (3) are interchanged, we have

$$
\begin{aligned}
& \text { (4) } \quad c: d=a: b, \\
& \text { (5) } \quad d: c=b: a, \\
& \text { (6) } \quad b: d=a: c .
\end{aligned}
$$

Form (2) is said to be obtained from form (1) by inversion, and form (3) from form (1) by alternation.

\section{EXERCISES}

1. How are (5) and (6) obtained from (4)?

2. Deduce forms (4), (5), and (6) from the essential property.

235. It should be remembered that the two terms of any ratio must be magnitudes of the same kind, since magnitudes of different kinds cannot be compared. But it is not necessary that the two ratios involved in a proportion should relate to magnitudes of the same kind, since the value of any ratio is a pure number, and the proportion merely expresses the equality of the two numbers.

For example, a proportion among four abstract numbers,

$$
4: 6=10: 15 \text {, }
$$

may be written in any one of the six forms of the preceding article. But if the terms involved are concrete, while we may write

or

$$
4 \text { men : } 6 \text { men }=10 \text { apples : } 15 \text { apples, }
$$$$
6 \text { men : } 4 \text { men }=15 \text { apples : } 10 \text { apples, }
$$

there would be no meaning in the statement,

$$
4 \text { men }: 10 \text { apples }=6 \text { men }: 15 \text { apples, }
$$

since no ratio can exist between magnitudes of different kinds. 
Hence, when a proportion exists among four quantities, two of one sort and two of another, care should always be taken to express the ratios between the pairs of quantities of the same sort.

236. Definition. If three quantities, $a, b, c$, are so related that $a: b=b: c$, they are said to be in continued proportion, and $b$ is called the mean proportional between $a$ and $c$, while $c$ is called the third proportional to $a$ and $b$.

In such a relation $b^{2}=a c$.

So also if $a, b, c, d, e, \ldots$ are so related that $a: b=b: c=$ $c: d$, etc., these quantities are said to be in continued proportion.

237. Theorem. If any four quantities, $a, b, c, d$ are in proportion, then (1) $a+b: b=c+d: d$; (2) $a-b: b=c-d: d$; and (3) $a+b: a-b=c+d: c-d$.

Proof. (1) If $a: b=c: d$, then

$$
\frac{a}{b}=\frac{c}{d}
$$

Adding one to both sides gives

$$
\begin{aligned}
& \frac{a}{b}+1=\frac{c}{d}+1 \\
& \frac{a+b}{b}=\frac{c+d}{d} .
\end{aligned}
$$

Therefore

$$
a+b: b=c+d: d \text {. }
$$

This relation is said to be derived from the given relation by Composition.

(2) Similarly, $\quad \frac{a}{b}-1=\frac{c}{d}-1$,

or

$$
\frac{a-b}{b}=\frac{c-d}{d} \text {. }
$$

Therefore

$$
a-b: b=c-d: d \text {. }
$$


This relation is said to be derived from the given relation by Division.

(3) Dividing each member of equation $(A)$ by the corresponding member of equation $(B)$, we have

$$
\begin{aligned}
& \frac{\frac{a+b}{b}}{\frac{a-b}{b}}=\frac{\frac{c+d}{d}}{\frac{c-d}{d}} \\
& \frac{a+b}{a-b}=\frac{c+d}{c-d}
\end{aligned}
$$

Therefore $a+b: a-b=c+d: c-d$.

This relation is said to be derived from the given relation by Composition and Division.

If $a: b=c: d$, show that

\section{EXERCISES}

$$
\begin{aligned}
& \text { (1) } m a: n b=m c: n d \\
& \text { (2) } a: a \pm b=c: c \pm d, \\
& \text { (3) } m a: m a \pm n b=m c: m c \pm n d, \\
& \text { (4) } a^{2}: b^{2}=c^{2}: d^{2} \\
& \text { (5) } a^{n}: b^{n}=c^{n}: d^{n}
\end{aligned}
$$

238. Theonem. If three terms of one proportion are equal, respectively, to the three corresponding terms of another, their fourth terms must be equal.

That is, if

and

then

$$
\begin{aligned}
a: b & =c: d, \\
a: b & =c^{\prime}: d, \\
c & =c^{\prime} .
\end{aligned}
$$

Proof. Since the ratios $c: d$ and $c^{\prime}: d$ are each equal to $a: b$, therefore, $c: d=c^{\prime}: d$, and $c$ must equal $c^{\prime}$. 
239. Theorem. If $P: Q$ equals $h: k$, and $Q: R$ equals $m: n$, then $P: R$ equals $h m: k n$.

For,

$$
\text { if } \frac{P}{Q}=\frac{h}{k} \text {, then } P=\frac{k}{h} \cdot Q \text {; }
$$

and,

$$
\text { if } \frac{Q}{R}=\frac{m}{n} \text {, then } Q=\frac{m}{n} \cdot R \text {. }
$$

Therefore, $P=\frac{h}{k} \cdot \frac{m}{n} \cdot R$, or $\frac{P}{R}=\frac{h \cdot m}{k \cdot n}$, that $P: R=h m: k n$.

240. Theorem. If any number of ratios are equal, then the sum of all the antecedents is to the sum of all the consequents as any one antecedent is to its consequent.

Let $a_{1}: b_{1}=a_{2}: b_{2}=a_{3}: b_{3}=\cdots$.

It is required to prove that

$$
a_{1}+a_{2}+a_{3}+\cdots: b_{1}+b_{2}+b_{3}+\cdots=a_{1}: b_{1} \text {, or } a_{2}: b_{2} \cdots \text {. }
$$

Suppose $r$ to be the common value of the given ratios, so that

$$
\begin{aligned}
& a_{1}: b_{1}=r, \text { then } a_{1}=r b_{1}, \\
& a_{2}: b_{2}=r, \text { then } a_{2}=r b_{2}, \\
& a_{3}: b_{3}=r, \text { then } a_{3}=r b_{3}, \\
& \text { ete., } \quad \text { etc. }
\end{aligned}
$$

Adding, we have $a_{1}+a_{2}+a_{3}+\cdots=r b_{1}+r b_{2}+r b_{3}+\cdots$

$$
=\left(b_{1}+b_{2}+b_{3}+\cdots\right) r \text {. }
$$

Therefore, $\quad \frac{a_{1}+a_{2}+a_{3}+\cdots}{b_{1}+b_{2}+b_{3}+\cdots}=r=\frac{a_{1}}{b_{1}}=\frac{a_{2}}{b_{2}}=\cdots$,

or

$$
a_{1}+a_{2}+a_{3}+\cdots: b_{1}+b_{2}+b_{3}+\cdots=a_{1}: b_{1}=a_{2}: b_{2}=\cdots \text {. }
$$

241. While it requires four quantities to form a proportion, we sometimes speak of one quantity being proportional to another. When we say that $A$ is proportional to $B$, we mean 
that $A$ and $B$ are connected in some way so that if $A$ changes to $A^{\prime}, B$ will change to a value $B^{\prime}$ such that

or

$$
\begin{aligned}
& A: A^{\prime}=B: B^{\prime}, \\
& A: B=A^{\prime}: B^{\prime} .
\end{aligned}
$$

The same thing may be expressed by saying that the ratio $A: B$ is constant.

Suppose

Then
$A: B=m$, a constant.

$A=m B$,

and any change in $A$ will cause a proportional change in $B$, or, what is the same thing, we may say $A$ varies as $B$.

If, on the other hand, the product of $A$ and $B$ is constant, that is,

$$
A B=m \text {, a constant, }
$$

then any increase or decrease in $A$ will cause a proportional decrease or increase in $B$, and we say that $A$ is inversely proportional to $B$, or that $A$ varies inversely as $B$.

\section{EXERCISES}

1. What number bears the same ratio to 6 as 5 bears to 10 ? As 3 to 9 ? As 6 to 9 ?

2. Find the fourth proportional to $5,8,10$; also the fourth proportional to $10,5,8$.

Definition. If $a, b, c, d$ are in proportion, $d$ is called the fourth proportional to $a, b$, and $c$.

3. If $a: b=c: d$, show that $3 a+2 b: a=3 c+2 d: c$.

4. If $a: b=2$, and $b: c=5$, show that $a: c=10$.

5. Find the mean proportional between 4 and 9 , between 2 and 32 , between 4 and 36, between 5 and 125 .

6. If $A$ varies as $B$, and when $A=10, B=4$, what is the value of $A$ when $B=14$ ?

7. If $A$ varies inversely as $B$, and when $A=2, B=9$, what is the value of $B$ when $A=3$ ?

8. If $a: b=b: c$, show that $a: c=a^{2}: b^{2}$. 


\section{SECTION II}

\section{SIMILAR POLYGONS}

\section{Proposition I}

242. A straight line parallel to one side of a triangle divides the other two sides in the same ratio.

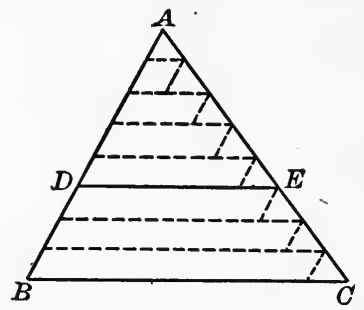

Let a straight line parallel to the base $B C$ of the triangle $A B C$ meet the other two sides at $D$ and $E$.

It is required to prove that $A D: D B=A E: E C$.

Proof. There are two cases to be considered, according as $A D$ and $D B$ are commensurable or incommensurable.

CASE I. When $A D$ and $D B$ are commensurable.

Let $A D$ and $D B$ have a common measure which is contained $m$ times in $A D$, and $n$ times in $D B$.

Then $A D: D B=m: n$.

Apply this measure to $A D$ and $D B$, and through the points of division draw lines parallel to $B C$, producing them to cut the side $A C$.

The number of segments thus formed in $A E$ and $E C$ is equal to the number in $A D$ and $D B$, respectively, namely, $m$ segments in $A E$, and $n$ in $E C$.

Through the several points of division in the side $A C$ draw lines parallel to $A B$. These are all equal. Why? 
Therefore the small triangles in the figure are identically equal, and the segments of $A C$ are all equal, (Arts. 94, 43.)

consequently

But

Therefore

$$
A E: E C=m: n \text {. }
$$$$
A D: D B=m: n \text {. }
$$

$$
A D: D B=A E: E C \text {. }
$$

(Axiom 1.)

CASE II. When $A D$ and $D B$ are incommensurable.

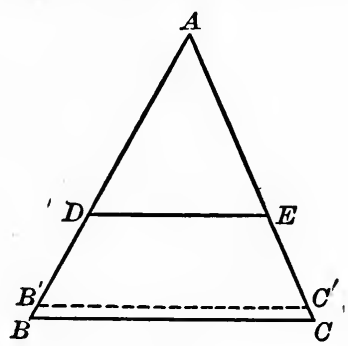

Take any measure of $A D$ for unit, and with it mark off segments on $A D$ and $D B$.

Since $A D$ and $D B$ are incommensurable, this unit will not measure $D B$ integrally, but will leave a remainder less than the unit, say $B^{\prime} B$.

Through $B^{\prime}$ draw a line parallel to $B C$, or to $D E$, meeting $A C$ at $C^{\prime \prime}$.

Then in $\triangle A B^{\prime} C^{\prime}$, since $A D$ and $D B^{\prime}$ are commensurable,

$$
\frac{A D}{D B^{\prime}}=\frac{A E}{E C^{\prime}}
$$

by Case I.

If now the length of the unit of measure be repeatedly diminished, $B^{\prime}$ will approach more and more nearly to $B$, and $C^{\prime}$ more and more nearly to $C$, since $B^{\prime} B$ is always less than the unit of measure; and in this way $B^{\prime} \dot{B}$ can be made less than any assigned quantity.

Therefore the ratio $\frac{A D}{D B^{\prime}}$ will approach as its limit the ratio $\frac{A D}{D B}$ 
And the ratio $\frac{A E}{E C^{\prime}}$ will approach as its limit the ratio $\frac{A E}{E C}$. But these ratios, $\frac{A D}{D B^{\prime}}$ and $\frac{A E}{E C^{\prime}}$, are always equal to each other.

Therefore their limits, $\frac{A D}{D B}$ and $\frac{A E}{E C}$, are equal. (Art. 230.)

That is,

$$
A D: D B=A E: E C \text {. }
$$

Noтe. The theorem of this proposition might be stated: A straight line parallel to one side of a triangle cuts the other two sides proportionally.

243. Corollary. In the diagram for this proposition

$$
\begin{aligned}
& A B: D B=A C: E C ; \\
& A D: A B=A E: A C ; \\
& A D: A E=A B: A C ;
\end{aligned}
$$

etc.

244. Converse of Proposition I. If a straight line divides two sides of a triangle in the same ratio, it is parallel to the third side.

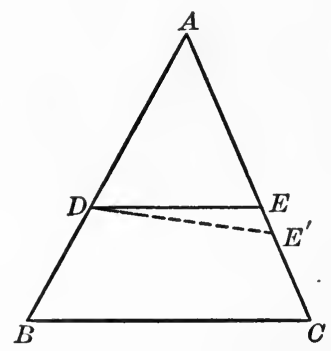

Let the straight line $D E$ divide the sides $A B$ and $A C$ of the triangle $A B C$ in the same ratio, so that

$$
A B: A D=A C: A E \text {. }
$$


If $D E$ is not parallel to $B C$, draw $D E^{\prime}$ parallel to $B C$.

Then

$$
A B: A D=A C: A E^{\prime} .
$$

But by hypothesis $A B: A D=A C: A E$.

Therefore $A E^{\prime}=A E$ (Art. 238), so that the lines $D E$ and $D E^{\prime}$ coincide.

Therefore $D E$ is parallel to $B C$.

\section{EXERCISES}

1. If two sides of a quadrilateral are parallel, any straight line drawn parallel to them will cut the other two sides, or these two sides produced, proportionally.

2. $A B C$ is a triangle, and through $D$, a point in $A B, D E$ is drawn parallel to $B C$, meeting $A C$ in $E$. Through $C$ a straight line $C F$ is drawn parallel to $B E$, meeting $A B$ produced in $F$. Prove that $A B$ is a mean proportional between $A D$ and $A F$, i.e. $A D: A B=A B: A F$.

3. The straight line which joins the mid-points of two sides of a triangle is parallel to the third side.

4. The straight line drawn through the mid-point of one side of a triangle parallel to a second side bisects the third side.

5. Any two straight lines cut by any three parallel straight lines are cut proportionally.

6. $B A C$ is any angle and $P$ any point within it. Through $P$ draw a straight line terminated both ways by the boundaries of the angle which is bisected at $P$.

Suggestion. Through $P$ draw $P D$ parallel to $B A$ meeting $A C$ in $D$. Find $E$ in $A C$ so that $A D=D E$. Join $E P$ and produce to meet $A B$ in $F . \quad E F$ is the required line.

7. $B A C$ is any angle and $P$ any point within it. Through $P$ draw a straight line terminated bath ways by the boundaries of the angle and divided in a given ratio at $P$.

Suggestion. Notice the difference between this problem and Ex. 6 . Follow the construction there given, making changes corresponding to the changes in the problem.

8. If a system of parallel lines intercept equal segments on one transversal, they will intercept equal segments on every other transversal. 


\section{Proposition II}

245. The bisector of an angle of a triangle divides the opposite side into segments proportional to the adjacent sides.

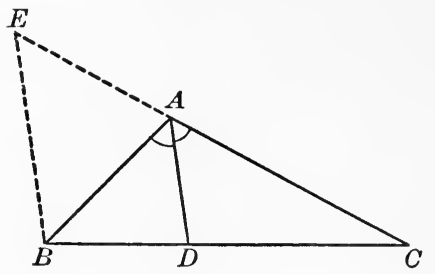

Let $A D$ bisect the angle $A$ of the triangle $A B C$, meeting the opposite side at $D$.

It is required to prove that $B D: D C=B A: A C$.

Proof. Through $B$ draw a line parallel to $D A$, meeting $C A$ produced at $E$.

Then

$$
\begin{aligned}
\angle A B E & =\angle B A D . \\
& =\angle C A D . \\
& =\angle A E B .
\end{aligned}
$$

Therefore

$$
A E=A B \text {. }
$$

Now

$$
B D: D C=E A: A C \text {. }
$$

Therefore $\quad B D: D C=B A: A C$.

\section{EXERCISES}

1. State and prove the converse of the theorem in Proposition II.

2. Apply this theorem to prove that the straight line bisecting the vertical angle of an isosceles triangle also bisects the base.

3. $A B C$ is any triangle whose base is bisected at $D$; the $\angle \mathrm{s} A D B$ and $A D C$ are bisected by straight lines meeting the sides $A B$ and $A C$ at $E$ and $F$, respectively. Prove that $E F$ is parallel to $B C$. 
4. Trisect a given line-segment.

Suggestion. Construct a triangle having the given line-segment, for base, and one of the sides double of the other. Bisect the vertical angle.

\section{Definitrons}

246. If $A B$ is a given line-segment and $C$ any point between $A$ and $B, C$ is said to divide $A B$ internally into the two segments $A C$ and $C B$. But if $C$ lies on the given line outside the segment $A B$,

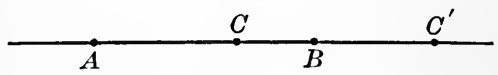
as at $C^{\prime \prime}$, then it is said to divide $A B$ externally into the segments $A C^{\prime}$ and $C^{\prime \prime} B$.

The two segments are the intercepts between the point and the extremities of the given line-segment, and their sum, proper attention being paid to signs, is always equal to the given linesegment. See Article 204.

With this definition the following theorem may be stated and proved similarly to that of Proposition II.

Theorem. The bisector of an external angle of a triangle divides the opposite side externally into segments proportional to the adjacent side; and conversely.

247. If the angles of one rectilinear figure taken in order are equal, respectively, to the angles of another rectilinear figure taken in order, the two figures are said to be mutually equiangular.

248. Two polygons are said to be similar when they have the same number of sides, are mutually equiangular, and have their pairs of corresponding sides proportional.

Two polygons of the same number of sides may in general be mutually equiangular without having their corresponding sides proportional ; or, they may have their corresponding sides proportional and not be mutually equiangular. Such polygons are not similar since they do not fulfil all the conditions of similarity.

Similar polygons are, so to speak, of the same shape but not necessarily of the same size. 


\section{Proposition III}

249. Two triangles are similar if the three angles of one are equal, respectively, to the three angles of the other.
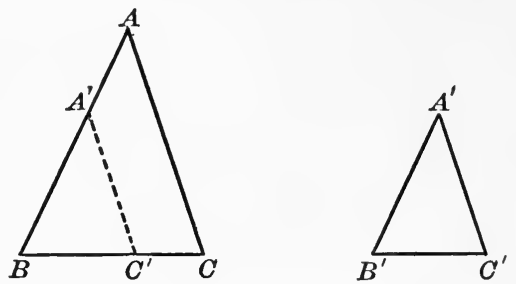

Let $A B C$ and $A^{\prime} B^{\prime} C^{\prime \prime}$ be two triangles in which the angle $A$ equals the angle $A^{\prime}, B$ equals $B^{\prime}$ and $C$ equals $C^{\prime}$.

It is required to prove that $A B: B C=A^{\prime} B^{\prime}: B^{\prime} C^{\prime}$,

$$
\begin{aligned}
& B C: C A=B^{\prime} C^{\prime \prime}: C^{\prime} A^{\prime}, \\
& C A: A B=C^{\prime \prime} A^{\prime}: A^{\prime} B^{\prime},
\end{aligned}
$$

or, more briefly, that $A B: B C: C A=A^{\prime} B^{\prime}: B^{\prime} C^{\prime \prime}: C^{\prime \prime} A^{\prime}$.

[Will this prove the triangles similar? Recall the definition of similar polygons.]

Proof. Place $\triangle A^{\prime} B^{\prime} C^{\prime}$ upon $\triangle A B C$ so that the vertex $B^{\prime}$ coincides with the vertex $B$ and $\angle A^{\prime} B^{\prime} C^{\prime \prime}$ with $\angle A B C$.

Then $A^{\prime}$ will fall on $B A$ or $B A$ produced, and $C^{\prime}$ will fall on $B C$ or $B C$ produced, and $A^{\prime} C^{\prime}$ will be parallel to $A C$. Why?

Therefore

$$
A B: B C=A^{\prime} B^{\prime}: B^{\prime} C^{\prime} \text {. }
$$

Similarly the other pairs of sides may be shown proportional, two and two.

250. Corollary. If two triangles have two angles of one equal, respectively, to two angles of the other, the triangles are similar. 


\section{Proposition IV}

251. If two triangles have an angle of one equal to an angle of the other, and the sides including these angles proportional, the triangles are similar.
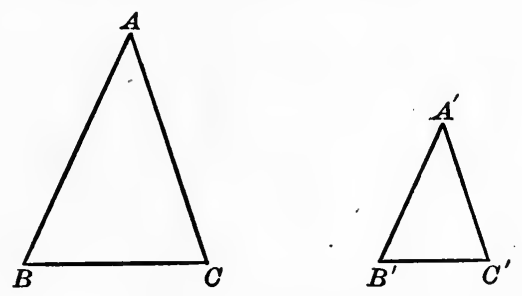

Let $A B C$ and $A^{\prime} B^{\prime} C^{\prime}$ be two triangles having the angle at $A$ equal to the angle at $A^{\prime}$, and the sides $A B$ and $A C$ proportional to $A^{\prime} B^{\prime}$ and $A^{\prime} C^{\prime}$, that is, having

$$
A B: A C=A^{\prime} B^{\prime}: A^{\prime} C^{\prime \prime} \text {. }
$$

It is required to prove that the two triangles are similar.

What do we need to show in order to prove the two triangles similar?

Proof. Suggestions. (1) Superpose the.triangles so that the equal angles coincide.

(2) The sides $B^{\prime} C^{\prime}$ and $B C$ are then parallel. Why?

(3) The triangles are therefore mutually equiangular and hence similar.

(Prop. III.)

\section{EXERCISES}

1. Two right triangles are similar if an acute angle of the one equals an acute angle of the other.

2. Two isosceles triangles are similar if their vertical angles are equal.

3. Two chords $A C$ and $B D$ of a circle $A B C$ intersect at $E$, either within or without the circle; prove $\triangle \mathrm{s} A E B$ and $C E D$ similar, and also $\triangle \mathrm{s} A E D$ and $B E C$.

4. The straight line joining the mid-points of two sides of a triangle is equal to half of the third side. 
252. Corollary to Proposition IV. If two similar polygons are divided into triangles by the diagonals drawn from a pair of homologous vertices, these triangles are similar each to each and are similarly placed.
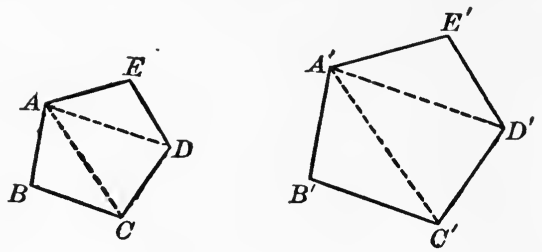

\section{Suggestions for Proof.}

First. By hypothesis polygons $A B C D E$ and $A^{\prime} B^{\prime} C^{\prime} D^{\prime} E^{\prime}$ are similar. That is $\angle B=\angle B^{\prime}$ and $A B: B C=A^{\prime} B^{\prime}: B^{\prime} C^{\prime}$.

Therefore $\triangle \mathrm{s} A B C$ and $A^{\prime} B^{\prime} C^{\prime}$ are similar.

Next.

$$
\begin{gathered}
\angle A C D=\angle A^{\prime} C^{\prime} D^{\prime} . \\
A C: C D=A^{\prime} C^{\prime}: C^{\prime} D^{\prime} .
\end{gathered}
$$

Why?

Why?

Therefore $\triangle \mathrm{s} A C D$ and $A^{\prime} C^{\prime} D^{\prime}$ are similar. And so on.

Conversely. If the diagonals drawn from one vertex in each of two polygons divide them into the same number of triangles, similar each to each and similarly placed, the two polygons are similar.

\section{EXERCISES}

1. State the theorem in the first chapter analogous to Proposition IV, proving the equality of two triangles.

2. From a point $E$ in the common base of the triangles $A C B$ and $A D B$ straight lines are drawn parallel to $A C$ and $A D$ meeting $B C$ and $B D$ in $F$ and $G$; show that $F G$ and $C D$ are parallel.

3. If two sides of a quadrilateral are parallel, the diagonals cut each other in the same ratio.

4. The bisector of an external angle of a triangle divides the opposite side externally into segments proportional to the adjacent sides, and conversely. 


\section{Proposition V}

253. If the ratios of the three sides of one triangle to the three sides of another, two and two, are equal, the triangles are similar.
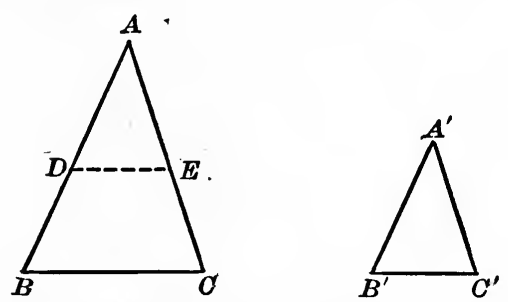

Let $A B C$ and $A^{\prime} B^{\prime} C^{\prime \prime}$ be two triangles such that

$$
A B: A^{\prime} B^{\prime}=A C: A^{\prime} C^{\prime}=B C: B^{\prime} C^{\prime} \text {. }
$$

It is required to prove that the two triangles are similar.

What must be shown in order to prove the triangles similar?

Proof. On $A B$ mark off a length $A D$ equal to $A^{\prime} B^{\prime}$, and on $A C$, a length $A E$ equal to $A^{\prime} C^{\prime}$. Join $D E$.

Then because $A B: A D=A C: A E$

$$
\triangle A D E \text { is similar to } \triangle A B C \text {; }
$$

(Prop. IV.)

Therefore

$$
A B: A D=B C: D E \text {. }
$$

But, by hypothesis, $A B: A^{\prime} B^{\prime}=B C: B^{\prime} C^{\prime}$, and by construction,

$$
\begin{aligned}
& A D=A^{\prime} B^{\prime} . \\
& D E=B^{\prime} C^{\prime} .
\end{aligned}
$$

(Art. 238.)

Hence $\triangle A D E$ is identically equal to $\triangle A^{\prime} B^{\prime} C^{\prime}$. (Art. 53.) But $\triangle A D E$ is similar to $\triangle A B C$.

Therefore $\triangle A^{\prime} B^{\prime} C^{\prime}$ is similar to $\triangle A B C$.

\section{EXERCISES}

1. State the analogous proposition on the equality of two triangles.

2. Two triangles are similar when their sides are respectively parallel or respectively perpendicular to each other.

3. If any quantities $A, B, C$, and $p, q, r$, are so related that $A: C=p: r$ and $B: C=q: r$, show that then $A+B: C=p+q: r$. 


\section{Proposition VI}

254. The perimeters of two similar polygons are in the same ratio as any two corresponding sides.

Suggestions for Proof.

1. Let $A B C D E \cdots$ and $A^{\prime} B^{\prime} C^{\prime} D^{\prime} E^{\prime} \cdots$ be any two similar polygons.

2. By definition $A B: A^{\prime} B^{\prime}=B C: B^{\prime} C^{\prime}=C D: C^{\prime} D^{\prime}$ etc.

3. Applying Art. 240, $A B+B C+C D+\cdots: A^{\prime} B^{\prime}+B^{\prime} C^{\prime}+C^{\prime} D^{\prime} \cdots=A B: A^{\prime} B^{\prime}$. Therefore

\section{Proposition VII}

255. If in a right triangle the perpendicular is drawn from the vertex of the right angle to the hypotenuse:

(1) The two triangles thus formed are similar to each other, and to the whole triangle;

(2) The perpendicular is a mean proportional between the segments of the hypotenuse;

(3) Each side of the triangle is a mean proportional between the hypotenuse and the segment adjacent to that side.

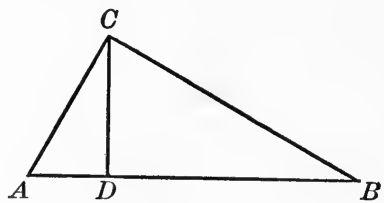

Let $A B C$ be a right triangle, right-angled at $C$, and $C D$ the perpendicular from $C$ to $A B$.

It is required to prove

(1) That the triangles $A C D$ and $C B D$ are similar to each other and to $A B C$.

All these triangles are easily shown to be mutually equiangular and therefore similar by Proposition III. 
(2) That

$$
A D: D C=D C: D B .
$$

This follows immediately from the fact that $\triangle \mathrm{s} A D C$ and $C D B$ are similar.

(3) That

and

$$
\begin{aligned}
& A B: A C=A C: A D, \\
& A B: B C=B C: B D .
\end{aligned}
$$

These relations follow from the fact that each small triangle is similar to the whole triangle.

256. Corollary. A perpendicular drawn from any point of a circle to a diameter is a mean proportional between the segments into which it divides the diameter.

\section{EXERCISES}

1. Show that the radius of a circle is a mean proportional between the segments of a tangent intercepted between its point of contact and any pair of parallel tangents.

2. If in two similar triangles perpendiculars are drawn from homologous vertices to the opposite sides, these are in the same ratio as any pair of homologous sides.

3. The sides of a triangle $A B C$ are 6,7 , and 8 feet, respectively ; find the lengths of the segments into which the bisector of the opposite angle will divide each side.

4. Show that the diagonals of any quadrilateral inscribed in a circle divide the quadrilateral into four triangles, which are similar, two and two.

5. Sum up the conditions under which two triangles are similar and compare these with the conditions under which two triangles are identically equal as given in Chapter I.

257. We shall hereafter frequently find it convenient to speak of the product of two line-segments, by which we shall mean the product of their measures by a common unit. With this understanding we may enunciate the following two theorems. 


\section{Proposition VIII}

258. If two chords of a circle intersect within the circle the product of the segments of the one equals the product of the segments of the other.

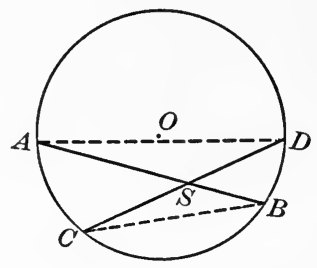

Let $A B$ and $C D$ be two chords intersecting at $S$ within a given circle.

It is required to prove that the product of the segments $A S$ and $S B$ equals the product of the segments $C S$ and $S D$.

Proof. Join $A D$ and $B C$.

$\triangle \mathrm{s} A S D$ and $C S B$ are mutually equiangular.

Therefore these triangles are similar, (Prop. III.) and

$$
A S: S D=C S: S B \text {. }
$$

Therefore the product $A S \cdot S B=$ the product $C S \cdot S D$.

(Art. 233.)

This theorem might be stated:

If two chords intersect within a circle, their segments are reciprocally proportional.

A segment of the first chord is to a segment of the second as the remaining segment of the second is to the remaining segment of the first; that is the segments are taken in reciprocal orders in the two ratios.

259. Corollary I. If through a fixed point within a circle any chord is drawn, the product of its segments is the same whatever its direction. 
260. Corollary II. Either segment of the least chord that can be drawn through a fixed point within a circle is a mean proportional between the segments of any other chord drawn through that point.

\section{Proposition IX}

261. If two chords of a circle, when produced, intersect without the circle, the product of the segments of the one equals the product of the segments of the other.

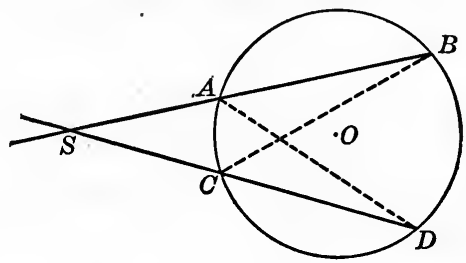

Let $A B$ and $C D$ be two chords intersecting at $S$ without the circle.

It is required to prove that the product of the segments $A S$ and $S B$ equals the product of the segments $C S$ and $S D$.

Proof. Join $A D$ and $B C$.

The proof is identical with that of Proposition VIII.

262. Corollary I. If a tangent and a secant of a circle intersect, the tangent is a mean proportional between the whole secant and its external segment.

This follows directly from the preceding proposition on considering a tangent as the limit of a secant, i.e. as a secant whose points of intersection coincide. Or it may easily be proved after the same general method as was used in the two preceding propositions, joining $T A$ and $T B$.

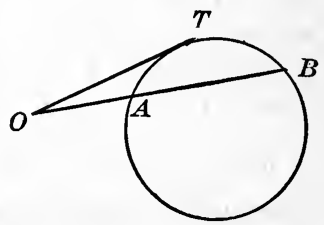


263. Corollary II. If from any point on the common chord of two intersecting circles produced, tangents are drawn to the circles, the lengths of these tangents are equal.

For either tangent is a mean proportional between the segments into which the chord is divided externally by the point.

Definition. The common chord of two intersecting circles produced indefinitely is called the Radical Axis of the circles; and a system of circles through the same two points is called a coaxial system, since all pairs of the circles have the same radical axis.

264. It will be interesting to recall the principle of continuity in connection with Propositions VIII and IX.

Proposition VIII states that if two chords $A B$ and $C D$ intersect within a circle at $S$, then $A S \cdot S B$ $=C S \cdot S D$.

Named in that order the two seginents of each chord are of the same sign.

Suppose. now we rotate the chord $C D$ about the point $D$. Then when $C$ coincides with $A$, $S$ will also coincide with $A$ and one segment of each chord will vanish. Both products $A S \cdot S B$ and $C S \cdot S D$ then become zero and are still equal.

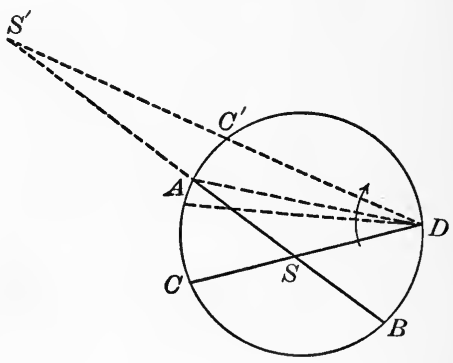

If we continue the rotation, $S$ will pass outside the circle, i.e. the two chords will intersect outside the circle, as in Proposition IX, and the point of intersection will divide them both externally. The two segments $A S$ and $S B$ will then be of opposite signs, as will also $C S$ and $S D$, and the products $A S \cdot S B$ and $C S \cdot S D$ still remain equal.

These two propositions may thus be comprised under a single more general theorem, as follows:

The products of the segments of any two chords of a circle, determined by their point of intersection, are equal. 


\section{EXERCISES}

1. If two line-segments intersect so as to make the product of the segments of one equal to the product of the segments of the other, their four extremities are concyclic.

Suggestion. Pass a circle through three of their extremities, and apply Proposition VIII.

2. If two circles intersect and through any point of their common chord two other chords are drawn, one in each circle, their four extremities are concyclic.

3. If two circles intersect, their common chord bisects their common tangents.

4. If two circles touch each other, the tangent at their point of contact bisects their other common tangents.

5. If three circles intersect two and two, their three common chords pass through one point.

Suggestion. If $A B$ and $C D$, the common chords of $X, Y$, and $X, Z$, intersect at $O$, and $P$, one of the common points of $Y$ and $Z$, is joined to $O$, then $P O$ must pass through the other common point of $Y$ and $Z$. If not let it cut $Y$ a second time at $Q$, and $Z$ a second time at $R$, and prove $O Q=O R$.

6. $A B$ is a given line-segment, $D$ and $E$ two points on it ; $D F$ and $E G$ are parallel and proportional to $A D$ and $A E$. Prove that $A, F$, and $G$ lie on one straight line.

7. The line-segment $A B$ is divided internally at $C$ and $D$, so that $A B: A C=A C: A D$. From $A$ any other line-segment $A E$ is drawn equal to $A C$. Prove that the triangles $A B E$ and $A E D$ are similar, and that $E C$ bisects the angle $B E D$.

265. We shall here insert a proposition which has no direct connection with the properties considered in this chapter, but which will serve as an additional example of a demonstration by the Method of Limits, and recall some of our fundamental notions of Ratio. It will be well for the pupil to review the proof of Proposition I in this connection. These two propositions are of the same general character, and the method used in demonstrating them will be frequently used in subsequent demonstrations. 


\section{Proposition X}

266. In equal circles, angles at the centre are in the same ratio as the arcs subtending them.
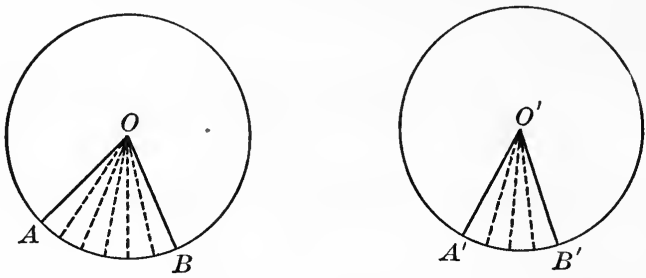

Let $A O B$ and $A^{\prime} O^{\prime} B^{\prime}$ be angles at the centres of two equal circles, subtended by the arcs $A B$ and $A^{\prime} B^{\prime}$, respectively.

It is required to prove

$$
\angle A O B: \angle A^{\prime} O^{\prime} B^{\prime}=\operatorname{arc} A B: \operatorname{arc} A^{\prime} B^{\prime} .
$$

Proof. Two cases arise according as the arcs $A B$ and $A^{\prime} B^{\prime}$ are commensurable or not.

Case I. Suppose $A B$ and $A^{\prime} B^{\prime}$ to have a common measure, which is contained $m$ times in $A B$ and $n$ times in $A^{\prime} B^{\prime}$. Apply this measure to the ares and mark the points of division.

Draw radii to the points of division.

These radii divide $\angle A O B$ into $m$ equal parts and $\angle A^{\prime} O^{\prime} B^{\prime}$ into $n$ parts, equal to each other and to the parts of $\angle A O B$.

Then

and

$$
\frac{\operatorname{arc} A B}{\operatorname{arc} A^{\prime} B^{\prime}}=\frac{m}{n}
$$

$$
\frac{\angle A O B}{\angle A^{\prime} O^{\prime} B^{\prime}}=\frac{m}{n} \text {. }
$$

Therefore

$$
\frac{\angle A O B}{\angle A^{\prime} O^{\prime} B^{\prime}}=\frac{\operatorname{arc} A B}{\operatorname{arc} A^{\prime} B^{\prime}},
$$

or

$$
\angle A O B: \angle A^{\prime} O^{\prime} B^{\prime}=\operatorname{arc} A B: \operatorname{arc} A^{\prime} B^{\prime} \text {. }
$$


CAse II. Suppose $A B$ and $A^{\prime} B^{\prime}$ to be incommensurable. Take any measure of $A^{\prime} B^{\prime}$, say $x$, and apply it to $A B$ as often as possible. Let the last point of division be $C$, so that the arc $C B$ is less than $x$.
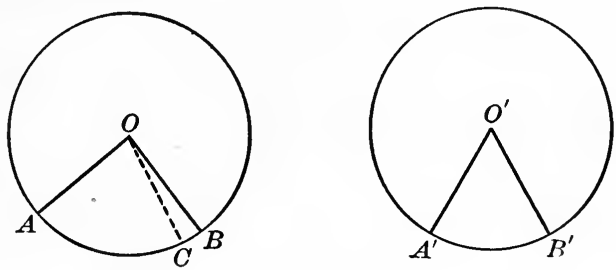

Then the arcs $A C$ and $A^{\prime} B^{\prime}$ are commensurable, so that

$$
\frac{\angle A O C}{\angle A^{\prime} O^{\prime} B^{\prime}}=\frac{\operatorname{arc} A C}{\operatorname{arc} A^{\prime} B^{\prime}}, \quad \text { by Case I. }
$$

By repeatedly decreasing the unit measure $x$, the arc $C B$ can be made smaller than any assigned quantity, so that the arc $A C$ will approach as its limit the arc $A B$, and at the same time $\angle A O C$ will approach as its limit $\angle A O B$.

Therefore the ratios $\frac{\angle A O C}{\angle A^{\prime} O^{\prime} B^{\prime}}$ and $\frac{\operatorname{arc} A C}{\operatorname{arc} A^{\prime} B^{\prime}}$ approach as their limits the ratios $\frac{\angle A O B}{\angle A^{\prime} O^{\prime} B^{\prime}}$ and $\frac{\operatorname{arc} A B}{\operatorname{arc} A^{\prime} B^{\prime}}$, respectively.

But $\frac{\angle A O C}{\angle A^{\prime} O^{\prime} B^{\prime}}=\frac{\operatorname{arc} A C}{\operatorname{arc} A^{\prime} B^{\prime}}$ for every unit measure $x$. Therefore the limit $\frac{\angle A O B}{\angle A^{\prime} O^{\prime} B^{\prime}}=$ the limit $\frac{\operatorname{arc} A B}{\operatorname{arc} A^{\prime} B^{\prime}}$.

(Art. 230.)

Or $\angle A O B: \angle A^{\prime} O^{\prime} B^{\prime}=\operatorname{arc} A B: \operatorname{arc} A^{\prime} B^{\prime}$.

267. Corollary I. In the same circle, angles at the centre are in the same ratio as the arcs subtending them.

268. Corollary II. In the same circle, or in equal circles, angles whose vertices lie on the circle are in the same ratio as the arcs subtending them. 


\section{Section III}

PROBLEMS OF CONSTRUCTION

\section{Proposition XI}

269. To divide a given line-segment into any required number of equal parts.

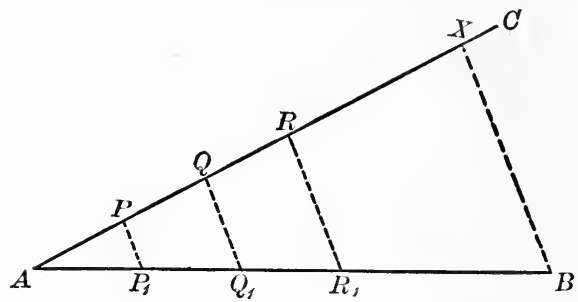

It is required to divide a given line-segment $A B$ into say $n$ equal parts.

Construction. From one end of the given line-segment draw any straight line $A C$ making an angle with $A B$.

On $A C$ mark off $n$ equal parts of any convenient length, beginning at the point $A$, and call the points of division $P, Q, R, \cdots$, the last one being $X$.

Join $X B$ and through the points of division $P, Q, R, \cdots$ draw lines parallel to $\mathrm{X} B$, cutting $A B$ in the points $P_{1}, Q_{1}, R_{1}, \ldots$.

Then $A B$ is divided into $n$ equal parts $A P_{1}, P_{1} Q_{1}, Q_{1} R_{1}, \cdots$.

Proof. Since $P P_{1}, Q Q_{1}, R R_{1}, \cdots, \mathrm{X} B$ are parallel and intercept equal segments on $A C$, they likewise intercept equal segments on $A B$.

(Prop. I.)

\section{EXERCISES}

1. Apply the method of this proposition to bisect a given line-segment, to trisect a given line-segment.

2. Show how to find three-fifths of a given line-segment. 


\section{Proposition XII}

270. To divide a given line-segment into parts proportional to other given line-segments.

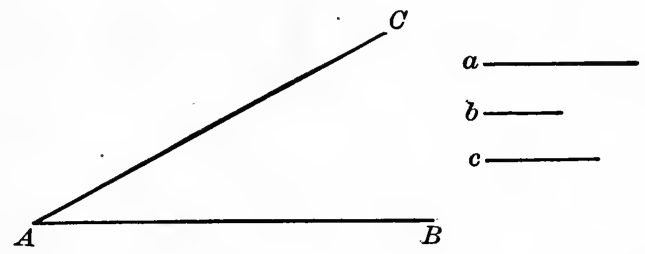

It is required to divide a given line-segment $A B$ into parts proportional to the given segments $a, b, c$.

The construction is similar to that for Proposition XI, except that on the arbitrary line $A C$, segments equal to $a, b, c$, respectively, are marked off, instead of segments equal to each other.

The pupil should write out the construction and proof in full.

271. If it is required to divide a given line-segment $A B$ in the ratio of $a$ to $b$, we must know whether the division is to be internal or external. If internal, proceed just as in the proposition above; but if external, mark on the line $A C$ the segment $A P$ equal to $a$, and $P Q$ equal to $b$ as before, but instead of $P Q$ being an extension of $A P$, it should lie upon $A P$ so that $A Q$ is divided externally at $P$ in the given ratio. Then join $Q B$ and draw $P M$ parallel to $Q B$. $\quad M$ will be the required point of division.

\section{EXERCISES}

1. Divide a given line-segment eight inches long into three parts proportional to one, two, and three.

2. Divide a given line-segment eight inches long (1) internally in the ratio $3: 2 ;(2)$ externally in the ratio $3: 2$. 


\section{Proposition XIII}

272. To find the fourth proportional to three given line-segments.

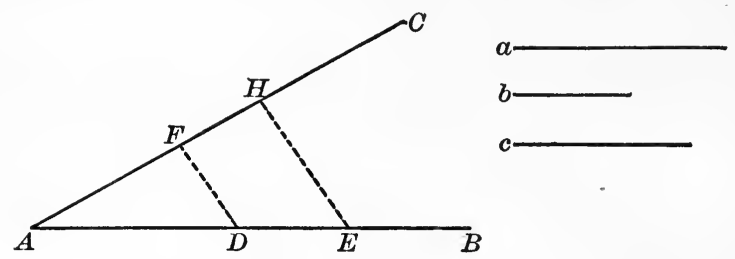

It is required to find the fourth proportional to the three given line-segments, $a, b, c$.

Construction. Draw two straight lines $A B$ and $A C$, making any angle with each other.

Upon $A B$ mark off the segments $A D$ and $D E$, equal respectively to $a$ and $b$.

Upon $A C$ mark off the segment $A F$ equal to $c$.

Join $D F$, and through $E$ draw $E H$ parallel to $D F$.

Then $F H$ is the fourth proportional to $a, b, c$.

The proof is apparent from Proposition I.

It should be noticed that if $a, b, c$ are taken in a different order the fourth proportional will be different.

\section{EXERCISES}

1. To find the third proportional to two given line-segments.

What is meant by a third proportional to two quantities?

2. How many different fourth proportionals can be found with three given line-segments?

3. $A B$ and $A C$ are two straight lines drawn from $A$. Produce $C A$ backward to $D$, making $A D=A C$; describe a circle through the three points $B, C, D$, and produce $B A$ to meet this circle at $E . A E$ is a third proportional to $A B$ and $A C$. 


\section{Proposition XIV}

273. To find the mean proportional between two given line-segments.

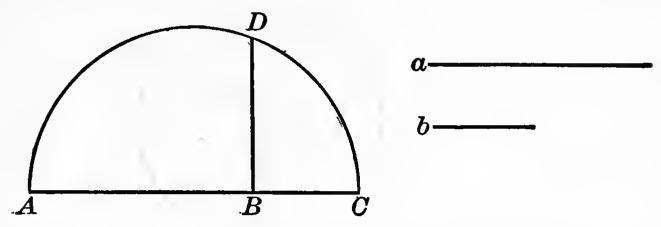

It is required to find the mean proportional between the two given line-segments $a$ and $b$.

Construction. Upon any straight line mark off the segments $A B$ and $B C$ equal to $a$ and $b$, respectively.

Upon $A C$ as diameter describe a circle, and at $B$ erect a perpendicular to $A C$, meeting the circle at $D$.

Then $B D$ is the mean proportional between $a$ and $c$.

(Art. 256.)

\section{EXERCISES}

1. If the given line-segments were $A C$ and $B C$, placed as in the diagram, show how to find a mean proportional between them.

2. Show that half the sum of two unequal line-segments is greater than the mean proportional between them.

274. Definition. When a given line-segment is divided into two parts such that one of the parts is a mean proportional between the whole segment and the other part, it is said to be divided in extreme and mean ratio.

For example, if $A B$ is divided internally at $C$ so that

$$
A B: A C=A C: C B,
$$

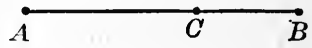

then $A B$ is divided internally in extreme and mean ratio; while if $C$ is so chosen on $A B$ produced that

$$
A B: B C=B C: A C,
$$

then $A B$ is divided externally in extreme and mean ratio. 


\section{Proposition XV}

275. To divide a given line-segment in extreme and mean ratio.

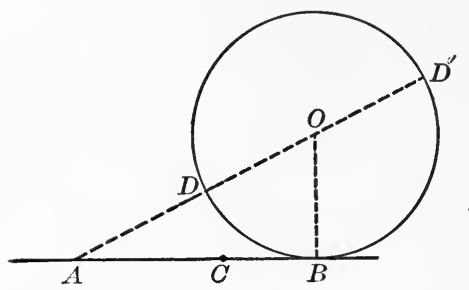

It is required to divide the line-segment $A B$ at a point $C$, so that

$$
A B: A C=A C: C B .
$$

Construction. At $B$ draw a straight line $B O$ perpendicular to $A B$ and equal to half of $A B$.

With centre $O$ and radius $O B$ describe a circle. Draw $A O$, cutting the circle at $D$ and $D^{\prime}$.

On $A B$ mark off a segment $A C$ equal to the shorter segment $A D$.

Then $C$ is the point required.

Proof. $A B$ is tangent to the circle $D B D^{\prime}$. (Art. 187.) Therefore $A D^{\prime}: A B=A B: A D$.or $A C$.

Whence, $A D^{\prime}-A B: A B=A B-A C: A C$. (Art. 237.)

Now

and also

$$
A B=2 O B=D D^{\prime} \text {, }
$$

$$
\begin{aligned}
A D^{\prime}-A B & =A D^{\prime}-D D^{\prime}=A D=A C ; \\
A B-A C & =B C .
\end{aligned}
$$

Therefore

$$
A C: A B=B C: A C,
$$

or, by inversion,

$$
A B: A C=A C: B C \text {. }
$$

\section{EXERCISE}

1. Suppose on $B A$ produced you should mark off a segment $A K$ equal to $A D^{\prime}$. Prove that $A B: A K=A K: B K$, or that $A B$ is divided externally at $D$ in extreme and mean ratio. 


\section{Proposition XVI}

276. Upon a given line-segment to construct a polygon similar to a given polygon, and such that the given line-segment shall be homologous to a given side.
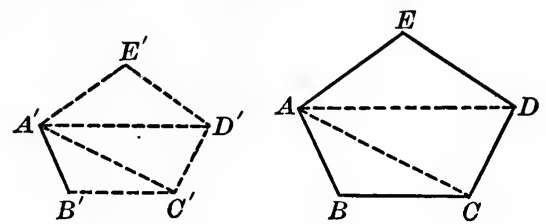

It is required to construct upon $A^{\prime} B^{\prime}$ a polygon similar to $A B C D E$, so that $A^{\prime} B^{\prime}$ shall be homologous to the side $A B$.

Construction. Divide the given polygon into triangles by drawing the diagonals from the vertex $A$.

Make $\angle \mathrm{s} A^{\prime} B^{\prime} C^{\prime}$ and $B^{\prime} A^{\prime} C^{\prime}$ equal to $\angle \mathrm{s} A B C$ and $B A C$, respectively.

Then $\triangle A^{\prime} B^{\prime} C^{\prime}$ is similar to $\triangle A B C$. (Prop. II.)

Similarly, construct

$\triangle A^{\prime} C^{\prime} D^{\prime}$ similar and similarly situated to $\triangle A C D$, and $\triangle A^{\prime} D^{\prime} E^{\prime}$ similar and similarly situated to $\triangle A D E$.

Then the polygon

$A^{\prime} B^{\prime} C^{\prime} D^{\prime} E^{\prime}$ is similar to the polygon $A B C D E$. (Art. 252.)

\section{EXERCISES}

1. Inscribe in a given circle a triangle similar to a given triangle.

Suggestion. At any point of the circle draw the tangent and chord making an angle equal to one angle of the given triangle. Then apply Article 198.

2. Circumscribe about a given circle a triangle similar to a given triangle. 


\section{Section IV}

\section{ADDITIONAL PROPOSITIONS}

\section{Proposition XVII}

277. If the sides of a triangle are cut by any straight line, the product of the ratios of their segments taken in order equals unity.

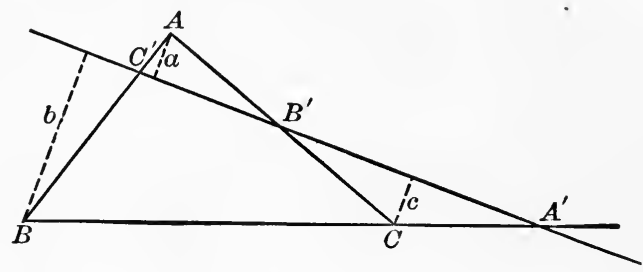

Let the sides $B C, C A, A B$, or these sides produced, be cut by a straight line in the points $A^{\prime}, B^{\prime}, C^{\prime \prime}$, respectively.

It is required to prove that $\frac{B A^{\prime}}{A^{\prime} C} \cdot \frac{C B^{\prime}}{B^{\prime} A} \cdot \frac{A C^{\prime}}{C^{\prime} B}=1$.

If we give heed to the signs of the segments, this product is - 1, since $B A^{\prime}$ and $A^{\prime} C$ are of opposite signs, while $C B^{\prime}$ and $B^{\prime} A, A C^{\prime}$ and $C^{\prime} B$ are of the same sign; that is, the first ratio is negative, the second and third positive. If the line cuts all three sides produced, the three ratios are all negative, and their product likewise is negative.

Proof. Draw perpendiculars $a, b, c$ from the vertices $A, B, C$, of the triangle, to the given transversal.

Then

$$
\frac{B A^{\prime}}{A^{\prime} C}=\frac{b}{c}, \frac{C B^{\prime}}{B^{\prime} A}=\frac{c}{a}, \quad \frac{A C^{\prime}}{C^{\prime} B}=\frac{a}{b} .
$$

Therefore

$$
\frac{B A^{\prime}}{A^{\prime} C} \cdot \frac{C B^{\prime}}{B^{\prime} A} \cdot \frac{A C^{\prime}}{C^{\prime} B}=\frac{b}{c} \cdot \frac{c}{a} \cdot \frac{a}{b}=1 \text {. }
$$

This theorem is attributed to Menelaus, a Greek geometer who lived in the latter part of the first century. 
278. Conversely. If on the sides $B C, C A, A B$ of a triangle $A B C$, three points, $A^{\prime}, B^{\prime}, C^{\prime}$, respectively, be so chosen as to fulfil the relation $\frac{B A^{\prime}}{A^{\prime} C} \cdot \frac{C B^{\prime}}{B^{\prime} A} \cdot \frac{A C^{\prime}}{C^{\prime} B}=-1$ (attention being paid to the signs of the segments), these three points are collinear.

Proof. Let the straight line $A^{\prime} B^{\prime}$ cut the side $A B$ of the triangle at $C_{1}^{\prime}$.

Then

$$
\frac{B A^{\prime}}{A^{\prime} C} \cdot \frac{C B^{\prime}}{B^{\prime} A} \cdot \frac{A C_{1}^{\prime}}{C_{1}^{\prime} B}=-1 \text { by the direct theorem. }
$$

But it was assumed that

$$
\frac{B A^{\prime}}{A^{\prime} C} \cdot \frac{C B^{\prime}}{B^{\prime} A} \cdot \frac{A C^{\prime}}{C^{\prime} B}=-1 .
$$

Therefore

$$
\frac{A C_{1}^{\prime}}{C_{1}^{\prime} B}=\frac{A C^{\prime}}{C^{\prime} B}
$$

whence $C^{\prime \prime}$ and $C_{1}^{\prime}$ coincide.

If attention were not paid to the signs of the segments, the last proportion could be true and $C^{\prime}$ and $C_{1}{ }^{\prime}$ not coincide, for in that case $C^{\prime}$ and $C_{1}{ }^{\prime}$ might divide the side $A B$ internally and externally in the same ratio.

\section{EXERCISES}

1. Points $E$ and $F$ are taken on the sides $A C$ and $A B$ of a triangle $A B C$, such that $A E$ is twice $E C$ and $B F$ is twice $F A$; the straight line $F E$ produced cuts the side $B C$ at $D$. Find the ratio of $B D$ to $D C$.

2. If the bisectors of the angles $B$ and $C$ of a triangle meet the opposite sides at $D$ and $E$, and if the straight line $D E$ produced meet $B C$ produced at $F$, then the external angle at $A$ is bisected by $A F$.

3. If a side $B C$ of a triangle $A B C$ is bisected by a straight line which meets the sides $A B$ and $A C$, produced if necessary, at $D$ and $E$ respectively, then $A E: E C=A D: D B$.

4. Two straight lines intersect at $A$, and $D$ is a point between them. Draw through $D$ a straight line such that the segments of it intercepted between $D$ and the given lines shall be in a given ratio.

Suggestion. Join $A D$ and produce to $E$ so that $A D: D E$ in the given ratio. Through $E$ draw a straight line parallel to one of the given lines, meeting the other at $H$. Then $H$ is a point of the required line. 


\section{Proposition XVIII}

279. If two triangles $A B C$ and $A^{\prime} B^{\prime} C^{\prime}$ are so situated that the straight lines joining the pairs of corresponding vertices $A, A^{\prime} ; B, B^{\prime} ; C, C^{\prime}$ are concurrent, then the points of intersection of the three pairs of corresponding sides are collinear.

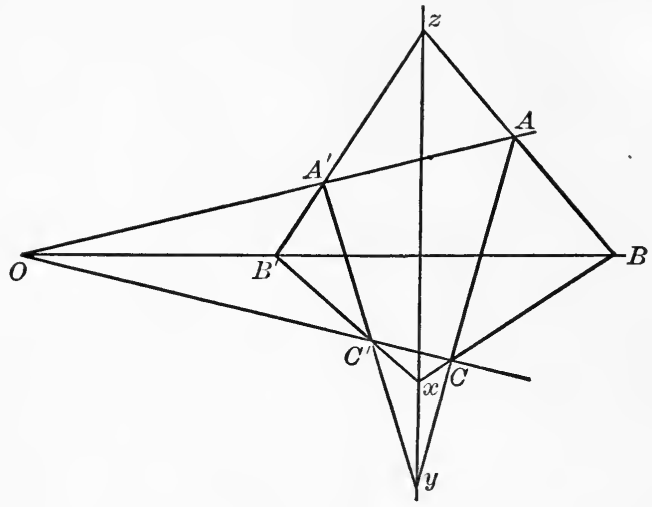

Let the two triangles $A B C$ and $A^{\prime} B^{\prime} C^{\prime}$ be so situated that the straight lines $A A^{\prime}, B B^{\prime}, C C^{\prime}$ meet in $O$.

It is required to prove that the points of intersection $x, y, z$, of the three pairs of corresponding sides $B C, B^{\prime} C^{\prime} ; C A, C^{\prime} A^{\prime}$; $A B, A^{\prime} B^{\prime}$, are collinear.

Proof. In order to prove what is stated it will be sufficient to show,

$$
\frac{A z}{z B} \cdot \frac{B x}{x C} \cdot \frac{C y}{y A}=-1
$$

For if this relation is satisfied, $x, y, z$, points on the sides of the triangle $A B C$, must be collinear.

(Art. 278.)

First, if we consider $\triangle O A B$ to be cut by the transversal $B^{\prime} A^{\prime} z$, we have

$$
\text { (1) } \frac{O A^{\prime}}{A^{\prime} A} \cdot \frac{A z}{z B} \cdot \frac{B B^{\prime}}{B^{\prime} O}=-1 \text {. }
$$


Similarly, from $\triangle \mathrm{s} O B C$ and $O C A$ cut by the transversals $B^{\prime} C^{\prime} x$ and $A^{\prime} C^{\prime \prime} y$, respectively, we get

$$
\begin{aligned}
& \text { (2) } \frac{O B^{\prime}}{B^{\prime} B} \cdot \frac{B x}{x C} \cdot \frac{C C^{\prime}}{C^{\prime} O}=-1 ; \\
& \text { (3) } \frac{A A^{\prime}}{A^{\prime} O} \cdot \frac{O C^{\prime}}{C^{\prime} C} \cdot \frac{C y}{y A}=-1 .
\end{aligned}
$$

Multiplying together the left-hand members of equations (1), (2), and (3), and also their right-hand members, we obtain

$$
\frac{A z}{z B} \cdot \frac{B x}{x C} \cdot \frac{C y}{y A}=-1 .
$$

Therefore $x, y, z$, are collinear.

280. Conversely. If two triangles $A B C$ and $A^{\prime} B^{\prime} C^{\prime \prime}$ are so situated that the points of intersection, $x, y, z$, of the three pairs of corresponding sides, $B C, B^{\prime} C^{\prime} ; C A, C^{\prime} A^{\prime} ; A B, A^{\prime} B^{\prime}$, are collinear, then the straight lines $A A^{\prime}, B B^{\prime}, C C^{\prime}$, joining the pairs of corresponding vertices, are concurrent.

This may be proved indirectly as follows:

Let $O$ be the intersection of the lines $B B^{\prime}$ and $C C^{\prime}$; and let the straight line $A O$ cut $B^{\prime} z$ at $A_{1}$, which, so far as we know, may or may not coincide with $A^{\prime}$.

Then $\triangle \mathrm{s} A B C$ and $A_{1} B^{\prime} C^{\prime \prime}$ fulfil the conditions of the direct theorem; therefore $A_{1} C^{\prime \prime}$ and $A C$ must intersect on the straight line $x z$.

But $A C$ cuts $x z$ at $y$.

Therefore $A_{1} C^{\prime \prime}$ must pass through $y$, and hence $A_{1}$ must coincide with $A^{\prime}$.

This theorem and its converse are known as Desargues's theorems, named from Gerard Desargues (born at Lyons, France, 1593, died 1662), to whom they are usually attributed, though the geometric properties involved in them were known much earlier. 


\section{Proposition XIX}

281. If through any point $O$ straight lines are drawn to the vertices $A, B, C$ of a triangle, meeting the opposite sides at $A^{\prime}, B^{\prime}, C^{\prime}$, respectively, then the product of the ratios of the segments of the sides taken in order equals unity, that is,

$$
\frac{B A^{\prime}}{A^{\prime} C} \cdot \frac{C B^{\prime}}{B^{\prime} A} \cdot \frac{A C^{\prime \prime}}{C^{\prime} B}=1 .
$$

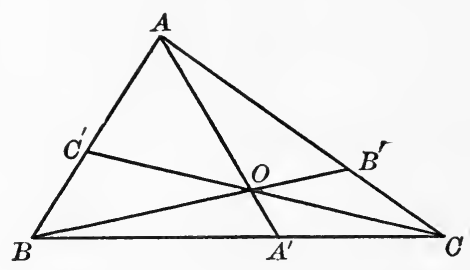

Proof. Take $\triangle A A^{\prime} C$ and consider $B O B^{\prime}$ a transversal of it.

Then

$$
\frac{A O}{O A^{\prime}} \cdot \frac{A^{\prime} B}{B C} \cdot \frac{C B^{\prime}}{B^{\prime} A}=-1 ;
$$

also from $\triangle A A^{\prime} B$ and the transversal $C O C^{\prime}$ we get

$$
\frac{A O}{O A^{\prime}} \cdot \frac{A^{\prime} C}{C B} \cdot \frac{B C^{\prime}}{C^{\prime} A}=-1 \text {. }
$$

Dividing the first of these results by the second, and remembering that $B C=-C B, A^{\prime} B=-B A^{\prime}$, etc., we obtain,

$$
\frac{B A^{\prime}}{A^{\prime} C} \cdot \frac{C B^{\prime}}{B^{\prime} A} \cdot \frac{A C^{\prime}}{C^{\prime} B}=1 .
$$

The converse of this theorem may easily be proved indirectly.

In the diagram we have chosen the point $O$ within the triangle $A B C$. The relation is equally true if the point is chosen outside the triangle. This should be verified both for a point $O$ obtained by passing out of the triangle across a side, and through a vertex. 


\section{Proposition XX}

282. To find the locus of a point whose distances from two fixed points are in a constant ratio different from unity.

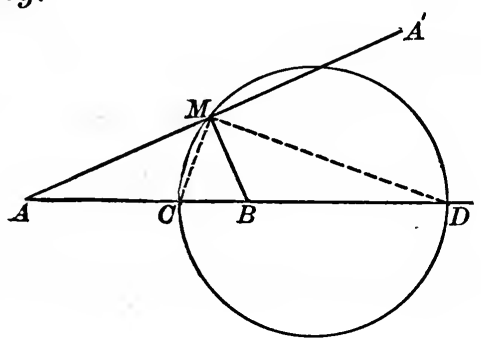

Let $A$ and $B$ be the two fixed points, and $M$ any point, such that $M A: M B$ equals a given fixed quantity $p$.

It is required to find the locus of $M$.

Construction. Join $A B$ and divide this line-segment internally at $C$ and externally at $D$ in the given ratio $p$, i.e. so that $A C: C B=A D: D B=p$.

Then $M A: M B=A C: C B$.

Therefore $M C$ bisects $\angle A M B$.

(Ex. 1, p. 160.)

Also $M A: M B=A D: D B$.

Therefore $M D$ bisects $\angle A^{\prime} M B$.

Therefore $\angle C M D$ is a right angle.

(Ex. 4, p. 164.)

Whence the locus of $M$ is a circle of which $C D$ is a diameter.

\section{EXERCISES}

1. What is the locus of a point equidistant from two fixed points? (See Art. 57.)

That is, what is the locus of a point whose distances from two fixed points are in a constant ratio, equal to unity?

2. How would the diagram for Proposition $\mathrm{XX}$ change if the given constant ratio were made more and more nearly equal to unity? As the ratio approaches unity how does the point $C$ move? How does the point $D$ move? What about the circle? 


\section{Harmonic Division*}

283. Defrnitron. Any line-segment which is divided internally and externally in the same ratio is said to be divided harmonically.

For example, if the segment $A B$, as in the last

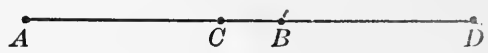
proposition, is divided internally at $C$ and externally at $D$ in such way that

$$
A C: C B=A D: D B,
$$

then $A B$ is divided harmonically at $C$ and $D$, and the four points $A, B, C, D$, are said to form a harmonic range.

284. Theorem. If any line-segment $A B$ is divided harmonically at $C^{Y}$ and $D$, the line-segment $C D$ is also divided harmonically at $A$ and $B$.

For if

$$
A C: C B=A D: D B
$$

so also is

$$
C A: A D=C B: B D,
$$

which shows that the segment $C D$ is divided at $A$ and $B$ internally and externally in the same ratio.

Thus in a harmonic range of four points there are two pairs of points such that each pair bears the same relation to the other pair.

The points of each pair are called 'harmonic conjugates' relative to the points of the other pair, and are said to be separated harmonically by them.

285. For the sake of symmetry we have written the ratios in the form $A C: C B=A D: D B$, but since the point $D$ divides the segment $A B$ externally, while $C$ divides it internally, one

* This section may be omitted on a first reading. 
of the four segments $A C, C B, A D, D B$, must be opposite in sign from the other three; and so, if attention be given to signs, we should write either

or

$$
\begin{aligned}
& A C: C B=A D:-D B, \\
& A C: C B=A D: B D .
\end{aligned}
$$

Choosing the latter form, we may write

$$
\frac{C B}{A C}=\frac{B D}{A D}
$$

or, since $C B=A B-A C$ and $B D=A D-A B$,

$$
\frac{A B-A C}{A C}=\frac{A D-A B}{A D} \text {. }
$$

Hènce

$$
\frac{A B}{A C}-1=1-\frac{A B}{A D},
$$

or

$$
\frac{A B}{A C}+\frac{A B}{A D}=2 \text {. }
$$

Therefore

$$
\frac{1}{A C}+\frac{1}{A D}=\frac{2}{A B}
$$

a formula of great value in the theory of Harmonic Division.

286. Another important relation among harmonic points on a line can be deduced in a similar way.

Let $A, C, B, D$ be a harmonic range, $A$ and $B$ being harmonically separated by

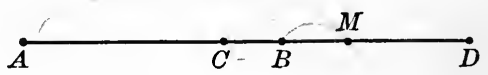
$C$ and $D$, and let $M$ be the mid-point of the line-segment $C D$.

Then

$$
A M \cdot B M=\overline{C M}^{2} \text {. }
$$

Proof. Since the points $A, C, B, D$ are harmonic, $A, B$ and $C, D$ being the pairs of conjugates,

$$
\frac{A C}{C B}=\frac{A D}{B D}
$$


Moreover, giving attention to the signs of segments,

$$
\begin{aligned}
& A C=A M+M C, \\
& C B=C M+M B, \\
& A D=A M+M D, \\
& B D=B M+M D
\end{aligned}
$$

and since $M$ is the mid-point of $C D$,

$$
C M=M D .
$$

Substituting in the relation

$$
\frac{A C}{C B}=\frac{A D}{B D},
$$

we have

$$
\frac{A M+M C}{C M+M B}=\frac{A M+M D}{B M+M D},
$$

or

$$
\frac{A M-C M}{C M-B M}=\frac{A M+C M}{B M+C M} .
$$

Whence, multiplying by the common denominator and collecting terms,

$$
A M \cdot B M=C M \cdot C M=\overline{C M}^{2} .
$$

\section{EXERCISES}

1. Show that two points of a straight line which are harmonically separated by two others are both on the same side of the mid-point of the line-segment determined by the two others.

2. The base of any triangle is cut harmonically by the bisectors of the internal and external vertical angles.

3. The hypotenuse of a right triangle is cut harmonically by two lines through the vertex of the right angle which make equal angles with one of the sides.

4. A straight line meets two intersecting circles at $P, Q, R, S$, and their common chord at $O$; prove that $O P, O Q, O R, O S$, taken in a proper order, are proportional. 


\section{MISCELLANEOUS EXERCISES}

1. If two triangles have one angle of the one equal to one angle of the other, and a second angle of the one supplementary to a second angle of the other, then the sides about the third angles are proportional.

2. $A E$ bisects the vertical angle of the triangle $A B C$ and meets the base in $E$; show that if circles are described about the triangles $A B E$ and $A C E$, their diameters are to each other in the same ratio as the segments of the base.

3. Two circles touch internally at $O ; A B$ a chord of the larger circle touches the smaller at $C$ which is cut by the lines $O A, O B$ at $P$ and $Q$; show that $O P: O Q=A C: C B$.

4. If two triangles have their sides parallel in pairs, the straight lines joining their vertices meet in a point, or are parallel.

5. If any two similar polygons have three pairs of corresponding sides parallel, the straight lines joining the corresponding vertices meet in a point or are parallel.

6. If $A, B, C, D$ are any four points on a circle and $E, F, G, H$ are the mid-points of the $\operatorname{arcs} A B, B C, C D, D A$, respectively, prove that the straight lines $E G$ and $F H$ are at right angles.

7. The sum of the perpendiculars drawn from any point within an equilateral triangle on the three sides is invariable.

8. Prove that the straight lines which trisect one angle of a triangle do not trisect the opposite side.

9. That part of any tangent to a circle which is intercepted between tangents at the extremities of a diameter is divided at the point of contact into segments such that the radius of the circle is a mean proportional between them.

10. If two chords $A B$ and $A C$, drawn from a point $A$ on a circle $A B C$, are produced to meet the tangent at the other extremity of the diameter through $A$, in the points $D$ and $E$ respectively, show that the triangle $A E D$ is similar to the triangle $A B C$.

11. On a circle of which $A B$ is a diameter take any point $P$. Draw $P C$ and $P D$ on opposite sides of $A P$ and equally inclined to it, meeting $A B$ at $C$ and $D$. Prove $A C: B C=A D: B D$. 


\section{SUMMARY OF CHAPTER III}

1. Definitions.

(1) To Measure - to find out by experiment how many times a given magnitude will contain a chosen unit. $\$ 211$.

(2) Multiple of a Given Magnitude - a magnitude which will contain that magnitude an integral number of times. $\$ 211$.

(3) Measure of a Given Magnitude - a magnitude which is contained in that magnitude an integral number of times. $\$ 211$.

(4) Commensurable Magnitudes - such as can be measured with a common unit. $\$ 213$.

(5) Incommensurable Magnitudes - such as cannot be measured by any common unit. $\$ 213$.

(6) Ratio of Two Quantities - their relative magnitude, i.e. how many times one is as great as the other. $\$ 216$.

(7) Ratio of Two Commensurable Magnitudes - the ratio of their numerical measures by a common unit. $\$ 220$.

(8) Ratio of Two Incommensurable Magnitudes - the limit which the ratio of their approximate measures by a common unit approaches, as this unit is indefinitely diminished. $\$ 229$.

(9) Limit of a Variable Quantity - a fixed quantity to which the variable approaches nearer than for any assignable difference, though it cannot be made absolutely identical with it. $\$ 227$.

(10) Proportion - a statement of the equality of two ratios. $\$ 232$.

(11) Continued proportion, mean proportional, third proportional. See $\S 236$.

(12) Mutually Equiangular Polygons - those having their angles equal, each to each, and in the same order. $\$ 247$.

(13) Similar Polygons - two polygons which have the same number of sides, are mutually equiangular, and have their pairs of corresponding sides proportional. \$248.

(14) Radical Axis of Two Intersecting Circles - the line of their common chord. $\$ 263$.

(15) Coaxial System of Circles - the circles through two fixed points. $\S 2(i 3$.

(16) Division in Extreme and Mean Ratio - division of a line-segment into two parts, such that one of them is a mean proportional between the whole segment and the other part. $\$ 274$.

(17) Harmonic Division-division of a line-segment internally and externally in the same ratio. $\S 283$. 


\section{Postulates.}

(1) If $P$ and $Q$ are any two equal magnitudes, and $R$ is a third magnitude of the same kind, then the ratio of $P$ to $R$ is equal to the ratio of $Q$ to $R$, i.e. if $P=Q$, then $P: R=Q: R$; and conversely, if $P$ and $Q$ are such that $P: R=Q: R$, then $P=Q$. (Postulate 6.) §218.

(2) If $P$ and $Q$ are two unequal magnitudes, and $R$ is a third magnitude of the same kind, then the ratio of $P$ to $R$ is greater or less than the ratio of $Q$ to $R$, according as $P$ is greater or less than $Q$. (Postulate 7.) $\quad \S 218$.

3. Problems.

(1) To divide a given line-segment into any required number of equal parts. $\$ 269$.

(2) To divide a given line-segment into parts proportional to other given line-segments. $\S 270$.

(3) To divide a given line-segment internally or externally in a given ratio. $\$ 271$.

(4) To find a fourth proportional to three given line-segments. $\S 272$.

(5) To find a mean proportional between two given line-segments. $\S 273$.

(6) To divide a given line-segment in extreme and mean ratio. $\$ 275$.

(7) Upon a given line-segment to construct a polygon similar to a given polygon, and such that the given line-segment shall be homologous to a given side. $\$ 276$.

(8) To find the locus of a point whose distances from two fixed points are in a constant ratio different from unity. $§ 282$.

4. Theorem on Limits.

If there are two variable quantities dependent on the same quantity in such a way that they remain always equal while each approaches a limit, then their limits are equal. $\S 230$.

5. Theorems on Proportion.

(1) If four numbers are in proportion, the product of the extremes equals the product of the means. $\S 233$.

(2) If $a: b=c: d$, then by inversion $b: a=d: c$, and by alternation $a: c=b: d . \quad \S 234$.

(3) If $a: b=c: d$, then by composition $a+b: b=c+d: d$, by division $a-b: b=c-d: d$, and by composition and division $a+b: a-b=c+d: c-d$. $\$ 237$. 
(4) If three terms of one proportion are equal, respectively, to the three corresponding terms of another, their fourth terms must be equal. $\$ 238$.

(5) If any number of ratios are equal, then the sum of all the ante. cedents is to the sum of all the consequents as any one antecedent is to its consequent. $\$ 240$.

\section{Theorems on the Similarity of Triangles.}

(1) Two triangles are similar if the three angles of one are equal, respectively, to the three angles of the other. $§ 249$.

(2) If two triangles have two angles of one equal, respectively, to two angles of the other, the triangles are similar. $\$ 250$.

(3) If two triangles have an angle of one equal to an angle of the other, and the sides including these angles proportional, the triangles are similar. $\$ 251$.

(4) If the ratios of the three sides of one triangle to the three sides of another, two and two, are equal, the triangles are similar. $\S 253$.

\section{Theorems on Chords of Circles.}

(1) If two chords of a circle intersect within the circle, the product of the segments of the one equals the product of the segments of the other. $\$ 258$.

(2) If through a fixed point within a circle any chord is drawn, the product of its segments is the same whatever its direction. $\S 259$.

(3) Either segment of the least chord that can be drawn through a fixed point within a circle is a mean proportional between the segments of any other chord drawn through that point. $\S 260$.

(4) A perpendicular drawn from any point of a circle to a diameter is a mean proportional between the segments into which it divides the diameter. $\$ 256$.

(5) If two chords of a circle, when produced, intersect without the circle, the product of the segments of the one equals the product of the segments of the other. $\$ 261$.

(6) If a tangent and a secant of a circle intersect, the tangent is a mean proportional between the whole secant and its external segment. $\$ 262$.

(7) If from any point on the common chord of two intersecting circles produced, tangents are drawn to the circles, the lengths of these tangents are equal. $\S 263$. 


\section{Miscellaneous Theorems.}

(1) A straight line parallel to one side of a triangle divides the other two sides in the same ratio, and conversely. $\$ \S 242, \dot{244}$.

(2) The bisector of an angle of a triangle divides the opposite side into segments proportional to the adjacent sides. $\$ 245$.

(3) If two similar polygons are divided into triangles by the diagonals drawn from a pair of homologous vertices, these triangles are similar each to each and are similarly placed, and conversely. $\S 252$.

(4) The perimeters of two similar polygons are in the same ratio as any two corresponding sides. $§ 254$.

(5) If in a right triangle the perpendicular is drawn from the vertex of the right angle to the hypotenuse: (1) the two triangles thus formed are similar to each other and to the whole triangle; (2) the perpendicular is a mean proportional between the segments of the hypotenuse ; (3) each side of the triangle is a mean proportional between the hypotenuse and the segment adjacent to that side. $\S 255$.

(6) In equal circles, angles at the centre are in the same ratio as the arcs subtending them. $\$ 266$.

(7) If the sides of a triangle are cut by any straight line, the product of the ratios of their segments taken in order equals unity, and conversely (Menelaus's theorem). $\$ \S 277,278$.

(8) If two triangles are so situated that the straight lines joining the pairs of corresponding vertices are concurrent, then the points of intersection of the three pairs of corresponding sides are collinear, and conversely (Desargues's theorem). $\$ \S 279,280$.

(9) If through any point straight lines are drawn to the vertices of a triangle, intersecting the sides, the product of the ratios of the segments of the sides taken in order equals unity. $\$ 281$.

(10) If any line-segment $A B$ is divided harmonically at $C$ and $D$, the line-segment $C D$ is also divided harmonically at $A$ and $B$. $§ 284$. 


\section{CHAPTER IV}

\section{AREAS OF PLANE POLYGONS}

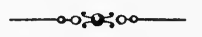

\section{SECTION I}

\section{PARALLELOGRAMS AND TRIANGLES}

287. In the preceding chapters we have discussed for the most part only geometrical figures (combinations of points and lines) and the conditions under which two such figures can be made to coincide. We have spoken of the length of a linesegment and of the relative lengths of two line-segments, of the size of an angle and of the relative sizes of two angles; but so far have had nothing to do with surfaces or the measurement of surfaces.

In this chapter we shall consider the measurement of surfaces enclosed by certain plane rectilinear figures.

288. When two polygons are placed so as to have one or more sides or parts of sides in common, without overlapping, they are said to be adjacent.

By disregarding the common parts of the boundaries
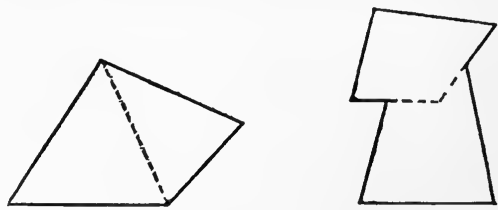
of two adjacent polygons, a third polygon is obtained which is defined to be the sum of the first two.

The sum of two polygons is thus a polygon such that within 
it lie all the points which are inside of either of the first two, and no other points.

If we have two polygons $A$ and $B$ whose sum as defined above is $C$, then the difference between the polygons $C$ and $A$ is the polygon $B$, or the difference between the polygons $C$ and $B$ is the polygon $A$.

289. Definition. The area of a plane closed figure is the surface lying within the figure.

The area of a circle is the surface lying within the circle, i.e. enclosed by the circle.

The area of a triangle is the surface enclosed by the three sides of the triangle.

Aхгом 11. If two plane polygons or other closed figures are identically equal, their areas are equal.

Aхгом 12. The sum of the areas of two plane polygons is equal to the area of their sum.

290. To measure a surface, we must first fix upon a unit of surface.

The unit most frequently chosen is the surface enclosed by a square whose sides are each of unit length.

If the unit length is one inch, the unit of surface is the surface enclosed by a square each of whose sides is an inch in length. Such a surface is called a square inch.

If the unit of length is a foot, then the unit of surface is a square foot; or, if the unit of length is a yard, or a centimetre, the unit of surface is a square yard, or a square centimetre, and so on.

291. The measure of the area of any geometrical figure is the number which expresses how many times this area will contain the chosen unit of surface.

For brevity we shall frequently speak of 'the area of a figure' as though it were 'the measure of the area,' and in that sense the area is a number.

Notatron. Hereafter the symbol $\square$ will frequently be used as an abbreviation for the word 'parallelogram,' $\square$ for the word 'rectangle,' and $\square$ for the word 'square.' 


\section{Proposition I}

292. Parallelograms upon the same base and between the same parallels are equal in area.
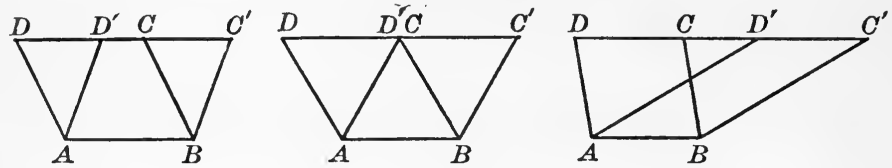

Let $A B C D$ and $A B C^{\prime} D^{\prime}$ be two parallelograms on the same base $A B$, such that the sides opposite to $A B$, viz. $C D$ and $C^{\prime} D^{\prime}$, are in the same straight line.

It is required to prove that these parallelograms are equal in area.

Proof. The whole polygon $A B C^{\prime} D$ is the sum of $\square A B C D$ and $\triangle B C^{\prime \prime} C$.

It is also the sum of $\square A B C^{\prime} D^{\prime}$ and $\triangle A D^{\prime} D$.

Therefore the area of $\square A B C D+$ the area of $\triangle B C^{\prime \prime} C=$ the area of $\square A B C^{\prime} D^{\prime}+$ the area of $\triangle A D^{\prime} D$.

But $\triangle \mathrm{s} B C^{\prime \prime} C$ and $A D^{\prime} D$ are identically equal. Prove.

Therefore the area of $\triangle B C^{\prime \prime} C=$ the area of $\triangle A D^{\prime} D$.

Hence the area of $\square A B C D=$ the area of $\square A B C^{\prime \prime} D^{\prime}$.

Notice that the proof applies equally well to any of the three possible cases represented in the diagrams, viz. (1) when $D C$ and $D^{\prime} C^{\prime}$ overlap, (2) when $C$ and $D^{\prime}$ coincide, (3) when $D C$ and $D^{\prime} C^{\prime}$ are separated.

293. When we say that two polygons are equal, we shall mean simply that they have equal areas, and not that they are necessarily superposable. If it is meant that two figures are superposable, we shall say as heretofore that they are $i d e n-$ tically equal.

294. Corollary I. Parallelograms upon equal bases and between the same parallels are equal in area. 
Let $A B C D$ and $A^{\prime} B^{\prime} C^{\prime} D^{\prime}$ be two parallelograms upon equal bases and between the same parallels. Join $A^{\prime} D$ and $B^{\prime} C$. $A^{\prime} B^{\prime} C D$ is also a parallelogram. Why?

Then $\square A B C D=\square A^{\prime} B^{\prime} C D$.

For they are upon the same base $C D$ and between the same parallels.

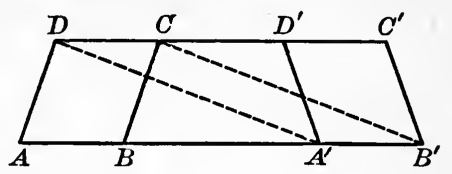

Similarly

$$
\square A^{\prime} B^{\prime} C D=\square A^{\prime} B^{\prime} C^{\prime} D^{\prime} .
$$

Therefore

$$
\square A B C D=\square A^{\prime} B^{\prime} C^{\prime} D^{\prime} \text {. }
$$

295. Corollary II. If a triangle and a parallelogram are upon the same base, or upon equal bases, and between the same parallels, the area of the triangle equals half the area of the parallelogram.

Let $\triangle A B E$ and $\square A B C D$ be upon the same, or equal bases, and between the same parallels.

It is required to prove that the area of the triangle equals half the area of the parallelogram. Complete $\square A B F E$.

Then $\square A B C D=\square A B F E$. (Prop. I.)

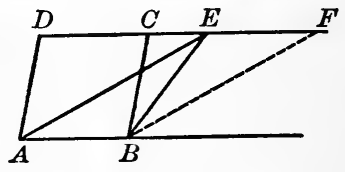

Also $\triangle A B E$ is identically equal to $\triangle F E B$ (Art. 124) and its area is therefore half the area of $\square A B F E$.

Therefore the area of $\triangle A B E$ equals half the area of $\square A B C D$.

296. Corollary III. Triangles upon the same base, or upon equal bases, and between the same parallels are equal in area.

297. Corollary IV. Triangles upon equal bases in the same straight line, having their opposite vertices in common, are equal in area.

\section{Definitions}

298. When one side of a parallelogram has been named the base, the perpendicular distance between it and the opposite side is called the altitude of the parallelogram. 
When one side of a triangle has been named the base, the perpendicular distance of the opposite vertex from it is called the altitude of the triangle.

299. Corollarx V. Any parallelogram is equal in area to a rectangle having an equal base and an equal altitude.

\section{EXERCISES}

1. The straight line joining any vertex of a triangle to the mid-point of the opposite side forms with the sides two triangles of equal areas.

2. Of two triangles which are between the same parallels, that has the greater area which has the greater base.

3. If two triangles, equal in area, are upon the same base and upon the same side of it, the straight line joining their vertices is parallel to the base.

4. $A B C$ is any triangle and $D E$ is drawn parallel to the base $B C$, meeting $A B$ and $A C$ at $D$ and $E ; B E$ and $C D$ are joined. Prove that $\triangle D B C=\triangle E B C, \triangle B D E=\triangle C E D$, and $\triangle A B E=\triangle A C D$.

5. $A B C D$ is a quadrilateral having $A B$ parallel to $C D$; its diagonals $A C$ and $B D$ intersect at $O$. Prove that $\triangle A O D=\triangle B O C$.

6. If through the vertices of a triangle, straight lines are drawn parallel to the opposite sides and produced to meet, the triangle so formed is the sum of four equal triangles.

7. On the same base and between the same parallels as a given parallelogram, construct a rhombus equal in area to the given parallelogram.

8. Divide a given parallelogram into two equal parallelograms. In how many ways can this be done?

9. $A B C$ is any triangle, and $D$ any point in $A B$. Find a point $E$ in $B C$ produced such that $\triangle D B E=\triangle A B C$.

10. If one diagonal of a quadrilateral bisects the other diagonal, it divides the quadrilateral into two triangles equal in area.

11. $A B C D$ is any quadrilateral, $A C$ and $B D$ its diagonals. A parallelogram $E F G H$ is constructed by drawing through the vertices straight lines parallel to the diagonals. Prove that $A B C D$ equals half of $E F G H$. 


\section{Proposition II}

300. The areas of two rectangles having equal altitudes are in the same ratio as their bases.
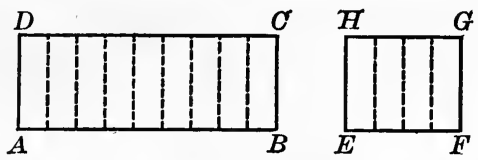

Let $A B C D$ and $E F G H$ be two rectangles of equal altitudes $A D$ and $E H$.

It is required to prove that

$$
\text { area of } A B C D \text { : area of } E F G H=A B: E F \text {. }
$$

Proof. There are two cases to be considered according as $A B$ and $E F$ are commensurable or incommensurable.

CASE I. When $A B$ and $E F$ are commensurable.

Let $A B$ and $E F$ have a common measure $x$ which is contained $m$ times in $A B$ and $n$ times in $E F$.

Then

$$
\frac{A B}{E F}=\frac{m}{n} .
$$

Divide $A B$ and $E F$ into segments each equal to $x$, and through the points of division draw lines parallel to $A D$ and $E H$, respectively, thus dividing the rectangle $A B C D$ into $m$ rectangles, and the rectangle $E F G H$ into $n$ rectangles, all identically equal (Art. 129), and therefore equal in area.

Since $\square A B C D$ is the sum of $m$ of these equal rectangles, and $\square E F G H$ is the sum of $n$ of them,

$$
\frac{\text { area of } A B C D}{\text { area of } E F G H}=\frac{m}{n} \text {. }
$$

Therefore

$$
\frac{\text { area of } A B C D}{\text { area of } E F G H}=\frac{A B}{E F} \text {, }
$$

or, area of $A B C D$ : area of $E F G H=A B: E F$. 
CAse II. When $A B$ and $E F$ are incommensurable.

Take any measure of $E F$, say $x$, and apply it to $A B$ as often as possible, letting $B^{\prime}$ be the last point of division, so that $B^{\prime} B$ is a remainder less than $x$.
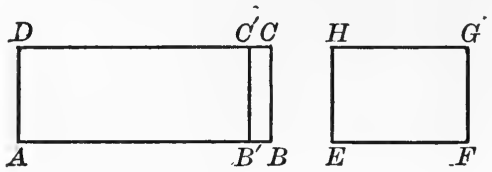

Through $B^{\prime}$ draw $B^{\prime} C^{\prime}$ parallel to $B C$.

Then $A B^{\prime} C^{\prime} D$ and $E F G H$ are two rectangles of equal altitudes whose bases $A B^{\prime}$ and $E F$ are commensurable.

Therefore

$$
\frac{\text { area of } A B^{\prime} C^{\prime} D}{\text { area of } E F G H}=\frac{A B^{\prime}}{E F} \text {. }
$$

If now the measure $x$ be repeatedly diminished, the base $A B^{\prime}$ approaches the base $A B$ as its limit, while the rectangle $A B^{\prime} C^{\prime} D$ approaches the rectangle $A B C D$ as its limit.

Therefore the limit of the variable ratio $\frac{\text { area of } A B^{\prime} C^{\prime} D}{\text { area of } E F G H}$ is the ratio $\frac{\text { area of } A B C D}{\text { area of } E F G H}$, and the limit of the variable ratio $\frac{A B^{\prime}}{E F}$ is the ratio $\frac{A B}{E F}$.

But these variable ratios are always equal; therefore their limits are equal. That is

$$
\frac{\text { area of } A B C D}{\text { area of } E F G H}=\frac{A B}{E F} \text {. }
$$

Or, area of $A B C D$ : area of $E F G H=A B: E F$.

301. Corollary I. The areas of two rectangles having equal bases are in the same ratio as their altitudes.

302. Corollary II. The areas of two triangles having equal altitudes are in the same ratio as their bases; or, having equal bases are in the same ratio as their altitudes. 


\section{Proposition III}

303. The areas of any two rectangles are in the same ratio as the products of their bases and altitudes.
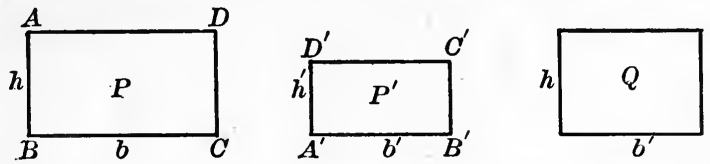

Let $A B C D$ and $A^{\prime} B^{\prime} C^{\prime} D^{\prime}$ be two rectangles whose areas are denoted by $P$ and $P^{\prime}$, whose bases are $b$ and $b^{\prime}$, and whose altitudes are $h$ and $h^{\prime}$, respectively.

It is required to prove that $\frac{P}{P^{\prime}}=\frac{b h}{b^{\prime} h^{\prime}}$.

Proof. Construct a third rectangle, denoting its area by $Q$, whose base is equal to $b^{\prime}$, and altitude equal to $h$.

Then

$$
\frac{P}{Q}=\frac{b}{b^{\prime}} \text { and } \frac{Q}{P^{\prime}}=\frac{h}{h^{\prime}} \text {. }
$$

Therefore

$$
\frac{P}{P^{\prime}}=\frac{b h}{b^{\prime} h^{\prime}}
$$

or

$$
P: P^{\prime}=b h: b^{\prime} h^{\prime} \text {. }
$$

\section{EXERCISES}

1. One rectangle has a base of $12 \mathrm{ft}$. and an altitude of $3 \mathrm{ft}$., another a base of $7 \mathrm{ft}$. and an altitude of $5 \mathrm{ft}$. What is the ratio of their areas ?

2. The ratio of the area of one rectangle to the area of another is $3: 2$. The first has an altitude of $8 \mathrm{ft}$. and a base of $15 \mathrm{ft}$. The second has an altitude of $5 \mathrm{ft}$. Find its base.

3. In tio rectangles, the base of the first is double that of the second, but the altitude of the first is only one-third that of the second; what is the ratio of their areas?

4. The lengths of two rectangular fields are in the ratio $2: 3$. What must be the ratio of their widths in order that the first may have an area twice as great as the second ? 
304. Suppose that in the last proposition the rectangle $A^{\prime} B^{\prime} C^{\prime \prime} D^{\prime}$ is a square having unit base and unit altitude.

Then $P^{\prime}$ is the unit of surface; in other words, it is unit area. And since $b^{\prime}$ and $h^{\prime}$ are in that case each unity, the

relation

$$
P: P^{\prime}=b h: b^{\prime} h^{\prime}
$$

becomes

$$
P: \text { unit area }=b h: 1 \text {, }
$$

or

$$
P=b h \text { times unit area. }
$$

That is, the area of the rectangle $A B C D$ is equal to the product of its base and altitude times unit area.

In other words, $P$ contains the unit area $b h$ times, or the measure of $P$ is $b h$.

Theorem. The measure of the area of a rectangle is equal to the product of its base and its altitude.

Or, more briefly, the area of a rectangle is equal to the product of its base and altitude.

Exercise. Divide a given rectangle into unit squares by straight lines drawn through points of division in its sides, and show that it contains as many squares as is represented by the product of its base and altitude.

305. Corollary I. The area of a parallelogram is equal to the product of its base and altitude.

For, any parallelogram is equal in area to a rectangle having the same base and an equal altitude.

(Art. 299.)

306. Corollary II. The area of a triangle is equal to half the product of its base and altitude.

For, the area of a triangle is half the area of a parallelogram having the same base and an equal altitude.

(Art. 295.)

307. Corollary III. The area of a square is equal to the square of any one of its sides. 


\section{Proposition IV}

308. The areas of two triangles having an angle of the one equal to an angle of the other are in the same ratio as the products of the sides containing the equal angles.

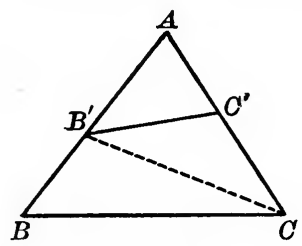

Let $B A C$ and $B^{\prime} A C^{\prime}$ be two triangles having the angles at $A$ equal.

It is required to prove that area of $B A C$ : area of $B^{\prime} A C^{\prime}=$ $B A \cdot A C: B^{\prime} A \cdot A C^{\prime}$.

Proof. Place the triangles so that the equal angles coincide as in the figure, and join $B^{\prime} C$.

Then

$$
\frac{\text { area of } B A C}{\text { area of } B^{\prime} A C}=\frac{B A}{B^{\prime} A},
$$

and

$$
\frac{\text { area of } B^{\prime} A C}{\text { area of } B^{\prime} A C^{\prime}}=\frac{A C}{A C^{\prime}} \text {. }
$$

Therefore

$$
\frac{\text { area of } B A C}{\text { area of } B^{\prime} A C^{\prime}}=\frac{B A \cdot A C}{B^{\prime} A \cdot A C^{\prime}},
$$

or area of $B A C$ : area of $B^{\prime} A C^{\prime}=B A \cdot A C: B^{\prime} A \cdot A C^{\prime}$.

309. Corollary. The areas of two parallelograms having an angle of the one equal to an angle of the other are in the same ratio as the products of the sides containing the equal angles.

\section{EXERCISE}

1. $A B C D$ is any parallelogram. Through $E$, any point in the diagonal $A C$, straight lines are drawn parallel to the sides. Show that the area of the parallelogram $E B$ equals the area of the parallelogram $E D$. 
310. It should be remembered that by the product of two line-segments we mean the product of their measures, and that by the square of a line-segment we mean the square of its measure.

Thus the product of two given line-segments equals the area of a rectangle whose adjacent sides are respectively equal to the given segments; and the square of a given line-segment equals the area of a square each of whose sides equals the given segment.

311. Theоnem. If a given line-segment is divided internally into any two parts, the square on the whole segment is equal in area to the sum of the squares on the two parts together with twice the rectangle contained by the two parts.

If we let $a$ and $b$ be the measures of the parts, then $(a+b)$ is the measure of the given segment and the theorem may be stated algebraically thus:

$$
(a+b)^{2}=a^{2}+b^{2}+2 a b .
$$

The accompanying diagram illustrates geometrically the truth of the theorem.

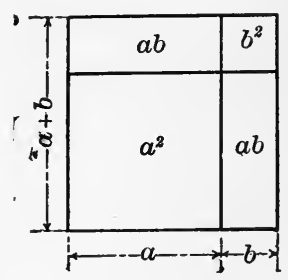

312. Theorem. If a given line-segment is divided externally into any two parts, the square on the given segment is equal in area to the sum of the squares on the two parts less twice the rectangle contained by the two parts.

As before, let $a$ and $b$ be the measures of the parts; then $(a-b)$ is the measure of the given segment, and the theorem may be stated algebraically thus:

$$
(a-b)^{2}=a^{2}+b^{2}-2 a b .
$$

Make a diagram illustrating this theorem. If $C$ divides $A B$ externally, and you let $A C=a$, and $B C=b$, then

$$
A B=a-b .
$$

Construct the squares on $a$ and $a-b$ producing the sides of the latter to meet the former. 
313. The relation between the two theorems stated above can be best shown by placing them side by side thus:

Theorem (1)

$$
\stackrel{A}{\overline{A B}^{2}=\overline{A C}^{2}+\overline{C B}^{2}+2 A C \cdot C B} .
$$

Theorem (2)

$$
\overrightarrow{A B}^{2}=\overline{A C}^{2}+\overrightarrow{C B}^{2}-2 A C \cdot C B .
$$

The two theorems are identical if we attach a sign to the segments. If $C$ divides the segment $A B$ internally, the parts $A C$ and $C B$ are of the same sign, while if $C$ divides $A B$ externally the parts are of opposite signs.

Thus the product $A C \cdot C B$ is in the first case positive, and in the second case negative. The square of either a positive or a negative segment is of course positive.

With this interpretation the relation

$$
\overline{A B}^{2}=\overline{A C}^{2}+\overline{C B}^{2}+2 A C \cdot C B
$$

is true no matter how the points $A, B, C$, are placed on a straight line.

This relation furnishes us with another illustration of the Principle of Continuity.

\section{EXERCISES}

1. Illustrate the following relations geometrically and state in words the theorem contained in each.

$$
\begin{array}{rlrl}
\text { (1) } & a(b+c) & =a b+a c . \\
\text { (2) } & a^{2}=4\left(\frac{a}{2}\right)^{2} . \\
\text { (3) } & a^{2}-b^{2}=(a+b)(a-b) . \\
\text { (4) } & (a+b)^{2}+(a-b)^{2} & =2 a^{2}+2 b^{2} .
\end{array}
$$$$
\text { (5) }(a+b)^{2}-(a-b)^{2}=4 a b \text {. }
$$ 


\section{EXERCISES}

1. The rectangle contained by two line-segments is equal to twice the rectangle contained by one of them and half of the other.

2. If a line-segment is divided internally into any three parts, the square on the whole segment is equal to the sum of the rectangles contained by the whole segment and its three parts.

3. Show that if a square and a rectangle have equal perimeters the square has the greater area.

4. If through the vertices of any triangle straight lines parallel to the sides are drawn to meet, two and two, the resulting triangle will contain three equal parallelograms.

5. Equal parallelograms on opposite sides of the same base are of equal altitude.

6. If two equal triangles are upon the same base and on opposite sides of it, the straight line joining their vertices is bisected by the base.

7. If a quadrilateral is divided into equal triangles by each of its diagonals, it is a parallelogram.

8. On the base of a given triangle construct another triangle of equal area having its vertex on a given straight line. In what case is this impossible?

9. The area of a trapezoid is equal to the product of its altitude and half the sum of its parallel sides.

10. Show that the sum of the squares on the two segments of a given line-segment is the least possible when it is bisected.

11. The sum of the squares on two internal segments of a given linesegment becomes greater as the point of section approaches one extremity.

12. Inscribe a square in a given semicircle.

13. If $A, C, D, B$ are four points on a straight line so situated that $D$ bisects $C B$, prove that the square on $A C$ is less than the sum of the squares on $A D$ and $D B$ by twice the rectangle $A D \cdot D B$.

14. If $B A C$ is any acute angle and $B D, C E$, are drawn perpendicular to its boundaries $A C, A B$, respectively, show that the rectangle whose sides are equal to $A B$ and $A E$ is equal in area to the rectangle whose sides are equal to $A C$ and $A D$.

15. If $A B C$ be a right-angled triangle and $C D$ be drawn perpendicular to the hypotenuse, then $A D: D B=\overline{A C}^{2}: \overline{B C}^{2}$. 


\section{SECTION II}

\section{AREAS OF SIMILAR POLYGONS}

\section{Proposition V}

314. The areas of similar triangles are in the same ratio as the squares of any two homologous sides.
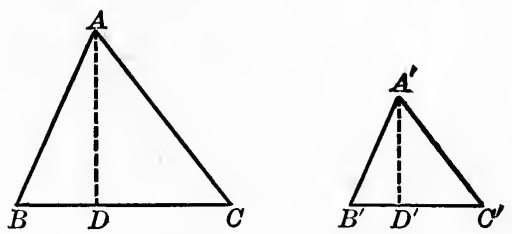

Let $A B C$ and $A^{\prime} B^{\prime} C^{\prime \prime}$ be two similar triangles, of which $A B$ and $A^{\prime} B^{\prime}$ are homologous sides.

It is required to prove that

$$
\text { area of } A B C \text { : area of } A^{\prime} B^{\prime} C^{\prime \prime}=\overline{A B}^{2}:{\overline{A^{\prime} B^{\prime}}}^{2} \text {. }
$$

Proof. Let $A D$ and $A^{\prime} D^{\prime}$ be the altitudes of the two triangles. Then $\frac{\text { area of } A B C}{\text { area of } A^{\prime} B^{\prime} C^{\prime}}=\frac{\frac{1}{2} B C \cdot A D}{\frac{1}{2} B^{\prime} C^{\prime \prime} \cdot A^{\prime} D^{\prime}}=\frac{B C}{B^{\prime} C^{\prime}} \cdot \frac{A D}{A^{\prime} D^{\prime}}$.

Now $\triangle \mathrm{s} A B C$ and $A^{\prime} B^{\prime} C^{\prime \prime}$ are similar;

therefore

$$
\frac{B C}{B^{\prime} C^{\prime}}=\frac{A B}{A^{\prime} B^{\prime}} \text {. }
$$

And since $\triangle \mathrm{s} A D B$ and $A^{\prime} D^{\prime} B^{\prime}$ are also similar, (Art. 249.)

$$
\frac{A D}{A^{\prime} D^{\prime}}=\frac{A B}{A^{\prime} B^{\prime}} \text {. }
$$

Therefore $\quad \frac{\text { area of } A B C}{\text { area of } A^{\prime} B^{\prime} C^{\prime}}=\frac{A B}{A^{\prime} B^{\prime}} \cdot \frac{A B}{A^{\prime} B^{\prime}}=\frac{\overline{A B}^{2}}{{\overline{A^{\prime} B^{\prime}}}^{2}}$.

\section{EXERCISES}

1. If the mid-points of two sides of a triangle are joined by a straight line, what part of the whole triangle is the smaller one so formed? 


\section{Proposition VI}

315. The areas of similar polygons are in the same ratio as the squares of any two homologous sides.
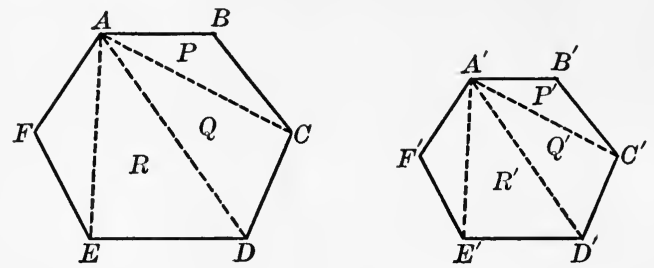

Let $A B C D E F$ and $A^{\prime} B^{\prime} C^{\prime} D^{\prime} E^{\prime} F^{\prime \prime}$ be any two similar polygons of which $A B$ and $A^{\prime} B^{\prime}$ are homologous sides.

It is required to prove that area of $A B \cdots F$ : area of $A^{\prime} B^{\prime} \cdots F^{\prime}=\overline{A B}^{2}:{\overline{A^{\prime} B^{\prime}}}^{2}$.

Proof. Divide the two polygons into triangles by drawing the diagonals from two homologous vertices, $A$ and $A^{\prime}$. These triangles will be similar, two and two.

(Art. 252.)

Let the areas of the triangles be $P, Q, R, S \cdots$ and $P^{\prime}, Q^{\prime}$, $R^{\prime}, S^{\prime}, \cdots$.

Then

$$
\begin{aligned}
& \frac{P}{P^{\prime}}=\frac{\overline{A B}^{2}}{{\overline{A^{\prime} B^{\prime 2}}}^{\prime}}, \quad \frac{Q}{Q^{\prime}}=\frac{{\overline{A C^{2}}}^{2}}{{\overline{A^{\prime} C^{\prime 2}}}^{2}}=\frac{\overline{A B}^{2}}{\bar{A}^{\prime} B^{\prime 2}}, \\
& \frac{R}{R^{\prime}}=\frac{\overline{A D}^{2}}{{\overline{A^{\prime} D^{\prime 2}}}^{2}}=\frac{{\overline{A C^{2}}}^{2}}{\bar{A}^{\prime} C^{\prime^{2}}}=\frac{\overrightarrow{A B^{2}}}{\bar{A}^{\prime} \bar{B}^{\prime 2}} \text {, etc. } \\
& \text { Whence } \quad \frac{P}{P^{\prime}}=\frac{Q}{Q^{\prime}}=\frac{R}{R^{\prime}}=\frac{S}{S^{\prime}}=\cdots=\frac{\overline{A B}^{2}}{{\overline{A^{\prime} B^{\prime}}}^{2}} \text {. }
\end{aligned}
$$$$
\text { Therefore } \frac{P+Q+R+S+\cdots}{P^{\prime}+Q^{\prime}+R^{\prime}+S^{\prime}+\cdots}=\frac{\overline{A B}^{2}}{\bar{A}^{\prime} B^{\prime 2}} \text {. }
$$

That is,

$$
\frac{\text { area of } A B \cdots F}{\text { area of } A^{\prime} B^{\prime} \cdots F^{\prime}}=\frac{\overline{A B}^{2}}{\bar{A}^{\prime} B^{\prime 2}} \text {. }
$$

316. Corollary. The areas of similar polygons are in the same ratio as the squares of any two homologous diagonals. 


\section{Proposition VII}

317. The area of the square described on the hypotenuse of a right triangle is equal to the sum of the areas of the squares described on the other two sides.

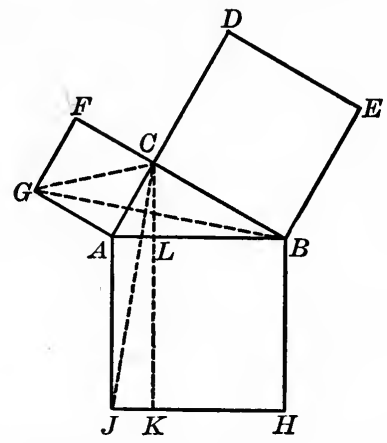

Let $A B C$ be a triangle, right-angled at $C$, and let $A H, B D$, and $C G$ be squares described upon the three sides.

It is required to prove that the area of the square $A H$ is equal to the sum of the areas of the two squares $B D$ and $C G$.

Proof. Draw $C K$ parallel to $A J$ or $B H$, and join $C J$ and $B G$. $F C$ and $C B$ are in the same straight line. Why?

Therefore $\triangle G A B=$ half of $\square G A C F$.

Also $\triangle A J C=$ half of $\square A J K L$.

But $\triangle \mathrm{s} G A B$ and $A J C$ or $C A J$ are identically equal. Why? Therefore $\square G A C F=\square A J K L$.

By joining $A E$ and $C H$, it may be similarly proved that $\square B E D C=\square B H K L$.

This statement should be fully verified by the pupil.

But $\square$ s $A J K L$ and $B H K L$ together make up $\square A J H B$.

Therefore the areas of $\square \mathrm{s} A C F G$ and $B E D C$ are together equal to the area of $\square A J H B$.

This theorem is usually attributed to Pythagoras, a Greek mathematician who lived about 550 B.c. 


\section{Alternate Proofs}

1. In the triangle $A B C$, right-angled at $C$, draw $C D$ perpendicular to the hypotenuse.

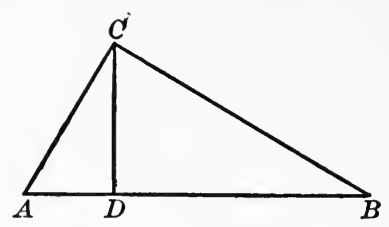

$$
\begin{array}{rlrl}
\text { Then } & A B: A C & =A C: A D, \\
\text { or } & & \overline{A C}^{2} & =A B \cdot A D . \\
\text { Also } & A B: B C & =B C: D B, \\
\text { or } & \overline{B C}^{2} & =A B \cdot D B .
\end{array}
$$

Therefore $\overline{A C}^{2}+\overline{B C}^{2}=A B \cdot A D+A B \cdot D B$

$$
\begin{aligned}
& =A B(A D+D B) \\
& =A B \cdot A B \\
& =\overline{A B}^{2} .
\end{aligned}
$$

2. With centre $A$ and radius $A C$ describe a circle.

Let the side $B A$ meet this circle in the points $E$ and $F$.

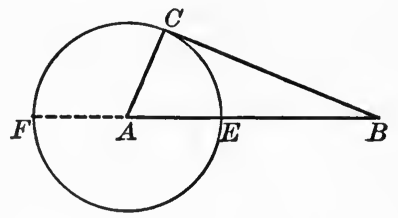

Then $B C$ is tangent to the circle. Why?

Hence

But

and

$$
B E \cdot B F=\overline{B C}^{2} \text {. }
$$

$$
B E=B A-E A=B A-A C,
$$

$$
B F=B A+A F=B A+A C .
$$


Therefore $B E \cdot B F=(B A-A C)(B A+A C)=\overline{B A}^{2}-\overline{A C}^{2}$.

Hence

That is,

$$
\overline{B A}^{2}-\overline{A C}^{2}=\overline{B C}^{2} \text {. }
$$

$$
\overline{B A}^{2}=\overline{A C}^{2}+\overline{B C}^{2} \text {. }
$$

Many other methods for proving this theorem have been devised. The proof presented in the main proposition is that found in Euclid's Elements. The nature of the proof given by Pythagoras is not certainly known.

318. Corollary. The area of any polygon described on the hypotenuse of a right triangle is equal to the sum of the areas of the similar polygons similarly described on the other two sides.

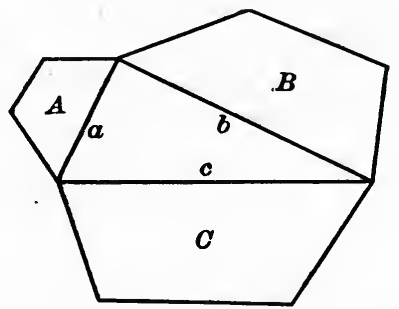

Let $a, b, c$, be the sides of the right triangle, and $A, B, C$, be the areas of the polygons similarly described on them.

Then

$$
A: C=a^{2}: c^{2} \text {. }
$$

$$
B: C=b^{2}: c^{2} \text {. }
$$

Therefore

$$
\begin{aligned}
A+B: C & =a^{2}+b^{2}: c^{2} . \\
a^{2}+b^{2} & =c^{2} ; \\
A+B & =C .
\end{aligned}
$$

But

therefore

\section{EXERCISES}

1. State and prove the converse of Proposition VII.

2. If the difference of the squares on two sides of a triangle is equal to the square on the third side, the triangle is right-angled.

3. The square described on the diagonal of a given square is equal to twice the given square. 
4. A quadrilateral is such that its diagonals intersect at right angles. Prove that the sum of the squares on one pair of opposite sides is equal to the sum of the squares on the other pair of opposite sides.

5. If $O$ is the point of intersection of the perpendiculars drawn from the vertices of a triangle $A B C$ to the opposite sides, the squares on $O A$ and $B C$ are together equal to the squares on $O B$ and $C A$, and also to the squares on $O C$ and $A B$.

6. If the hypotenuse and a side of one right triangle are equal to the hypotenuse and a side of another right triangle, show that the triangles are identically equal.

Prove by use of Proposition VII. See also a different proof in Chapter I, Proposition XIX.

7. Find a line-segment the square on which is equal to three times the square on a given line-segment.

8. Find a line-segment the square on which is equal to the difference of the squares on two given line-segments.

9. Five times the square on the hypotenuse of a right triangle is equal to four times the sum of the squares on the medians to the other two sides.

10. Three times the square on any side of an equilateral triangle is equal to four times the square on the perpendicular drawn from a vertex to the opposite side.

\section{PROJECTIONS}

319. If from a given point a perpendicular is drawn to a given straight line, the point of intersection, or, as we say, the foot of the perpendicular, is called the projection of the given point upon the given line.

The projection of the point $P$ upon the straight line $A B$ is the point $P^{\prime}$, and the projection of $Q$ is $Q^{\prime}$, if $P P^{\prime}$ and $Q Q^{\prime}$ are perpendicular to $A B$.

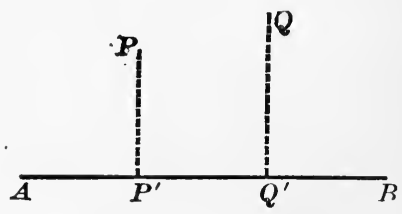

The projection of a given line-segment upon any straight line is the intercept between the projections of its extremities upon the line. 
The projection of the line-segment $P Q$ upon the straight line $A B$ is the segment $P^{\prime} Q^{\prime}$; the projection of the line-segment $R S$ upon $A B$ is the segment $R S^{\prime}$.

A line-segment is equal to its projection upon any straight line parallel to it, but is greater than its projection upon any straight line not parallel to it.

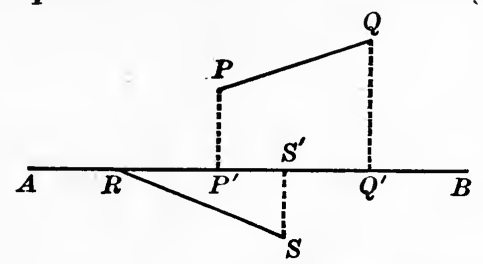

What is the projection upon a given straight line of a segment at right angles to it?

320. Theorem. If two line-segments $O A$ and $O B$ have one extremity in common, the product of $O A$ and the projection of $O B$ upon the line of $O A$ equals the product of $O B$ and the projection of $O A$ upon the line of $O B$.

From $A$ and $B$ draw the perpendiculars $A A^{\prime}$ and $B B^{\prime}$, respectively.

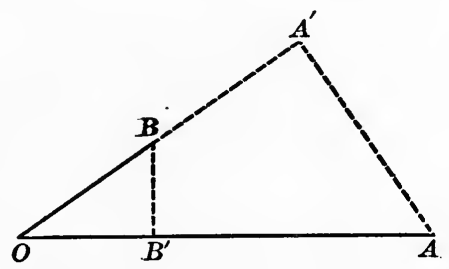

Then $O A^{\prime}$ is the projection of $O A$ upon the straight line $O B$, and $O B^{\prime}$ is the projection of $O B$ upon the straight line $O A$, and we are required to prove that $O A \cdot O B^{\prime}=O B \cdot O A^{\prime}$. $\triangle \mathrm{s} O A A^{\prime}$ and $O B B^{\prime}$ are similar. Why?

Therefore $O A: O A^{\prime}=O B: O B^{\prime}$, or $O A \cdot O B^{\prime}=O B \cdot O A^{\prime} . \quad($ See Ex. 14, p. 206.) 


\section{Proposition VIII}

321. In any triangle the square on the side opposite an acute angle is less than the sum of the squares on the other two sides by twice the product of either of these sides and the projection of the other upon it.
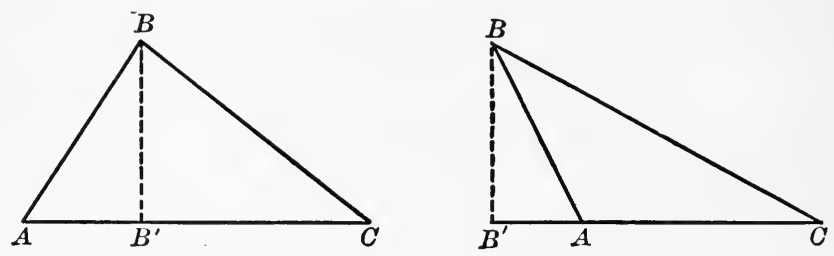

Let the angle $C$ of the triangle $A B C$ be acute, and from $B$ draw the perpendicular $B B^{\prime}$ to the line $A C$, so that $C B^{\prime}$ is the projection of $C B$ upon the line $A C$.

It is required to prove that

$$
\overrightarrow{A B}^{2}=\overline{A C}^{2}+\overrightarrow{C B}^{2}-2 A C \cdot C B^{\prime} .
$$

Proof. Since in either diagram $A B^{\prime}$ is divided externally at $C$,

$$
{\overline{A B^{\prime}}}^{2}={\overline{A C^{2}}}^{2}+{\overline{C B^{\prime}}}^{2}-2 A C \cdot C B^{\prime} .
$$

To each side of this relation add ${\overline{B B^{\prime}}}^{2}$.

Then $\overline{A B^{2}}+\overline{B B^{\prime}}={\overline{A C^{2}}}^{2}+{\overline{C B^{\prime}}}^{2}+\overline{B B^{\prime}}{ }^{2}-2 A C \cdot C B^{\prime}$.

But

$$
{\overline{A B^{\prime}}}^{2}+{\overline{B B^{\prime}}}^{2}=\overline{A B}^{2}
$$

and

$$
{\overline{C B^{\prime}}}^{2}+{\overline{B B^{\prime}}}^{2}=\overline{C B}^{2} \text {. }
$$

Therefore $\quad \overline{A B}^{2}=\overline{A C}^{2}+\overline{C B}^{2}-2 A C \cdot C B^{\prime}$.

What does this relation become in case $B^{\prime}$ coincides with $A$, i.e. in case $A$ is a right angle?

\section{EXERCISE}

If $A B$ and $C D$ are any two given line-segments, prove that the product of $A B$ and the projection of $C D$ upon the line of $A B$ equals the product of $C D$ and the projection of $A B$ upon the line of $C D$. 


\section{Proposition IX}

322. In an obtuse-angled triangle the square on the side opposite the obtuse angle is greater than the sum of the squares on the other two sides by twice the product of either of these sides and the projection of the other upon it.

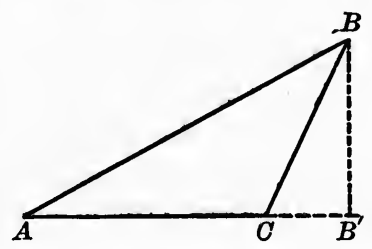

Let the angle $C$ of the triangle $A B C$ be obtuse, and from $B$ draw the perpendicular $B B^{\prime}$ to the line $A C$, so that $C B^{\prime}$ is the projection of $C B$ upon the line $A C$.

It is required to prove that

$$
\overline{A B}^{2}=\overline{A C}^{2}+\overline{C B}^{2}+2 A C \cdot C B^{\prime} .
$$

Proof. Since $A B^{\prime}$ is divided internally at $C$,

$$
{\overline{A B^{\prime}}}^{2}=\overline{A C}^{2}+{\overline{C B^{\prime}}}^{2}+2 A C \cdot C B^{\prime} \text {. }
$$

To each side of this relation add ${\overline{B B^{\prime}}}^{2}$.

Then ${\overline{A B^{\prime}}}^{2}+{\overline{B B^{\prime}}}^{2}={\overline{A C^{2}}}^{2}+{\overline{C B^{\prime}}}^{2}+{\overline{B B^{\prime}}}^{2}+2 A C \cdot C B^{\prime}$.

But

$$
{\overline{A B^{\prime}}}^{2}+{\overline{B B^{\prime}}}^{2}=\overline{A B}^{2}
$$

and

$$
{\overline{C B^{\prime}}}^{2}+{\overline{B B^{\prime}}}^{2}=\overline{C B}^{2} \text {. }
$$

Therefore $\quad \overline{A B}^{2}=\overline{A C}^{2}+\overline{C B}^{2}+2 A C \cdot C B^{\prime}$.

323. No doubt the pupil has already noticed the close similarity between the proofs of Propositions VIII and IX. In fact, except for the change of sign of the segment $C B^{\prime}$, the proofs are identical. 
We might with perfect propriety consider the theorem of Proposition IX a general theorem of which both Propositions VII and VIII are special cases, though, of course, the proof of Proposition IX must follow that of Proposition VII, since it depends upon it.

Suppose, for example, in the diagram for Proposition IX we rotate the side $C B$ about $C$ in the way indicated by the arrow-head. The angle $A C B$ gradually

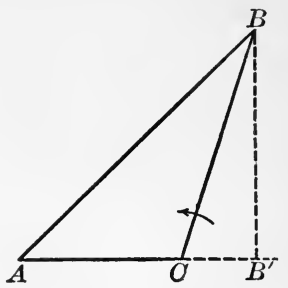
decreases in magnitude, while the point $B^{\prime}$ approaches $C$ and coincides with $C$ when $\angle A C B$ becomes a right angle.

In this position the segment $C B^{\prime}$ is zero and the product $2 A C \cdot C B^{\prime}$ is also zero.

The relation $\overline{A B}^{2}=\overline{A C}^{2}+\overline{C B}^{2}+2 A C \cdot C B^{\prime}$ thus becomes $\overline{A B}^{2}=\overline{A C}^{2}+\overline{C B}^{2}$,

which is the relation proved for a right triangle in Proposition VII.

If the rotation of $C B$ about $C$ continues, $\angle A C B$ becomes acute, $B^{\prime}$ moves in between $C$ and $A$, the segment $C B^{\prime}$ changes sign, and we have the relation

$$
A B^{2}=\overline{A C}^{2}+\overline{C B}^{2}-2 A C \cdot C B^{\prime},
$$

which was proved independently in Proposition VIII.

Here again is an illustration of the Principle of Continuity.

\section{EXERCISE}

The sum of the squares on two sides of any triangle is equal to twice the squares on half the third side, and on the median drawn to that side.

Suggestion. The median makes in general one acute angle and one obtuse angle with the third side. Apply Propositions VIII and IX, and add. 
The Area of a Triangle in Terms of its Three Sides

324. The area of a triangle has been found (Art. 306) to be half the product of its base and altitude, but it is sometimes more convenient to have at hand a formula expressing the area in terms of the lengths of the three sides. Such a formula we are now ready to deduce.
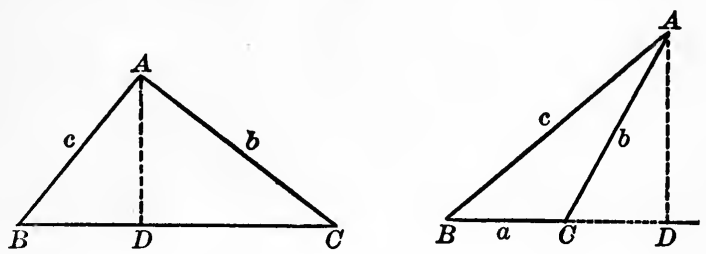

Letter the triangle $A B C$ symmetrically, denoting by $a$ the length of the side opposite the vertex $A$, by $b$ the length of the side opposite the vertex $B$, and by $c$ the length of the side opposite the vertex $C$.

- Giving proper attention to the sign of the segment $C D$,

$$
A B^{2}=\overline{B C}^{2}+\overline{C A}^{2}+2 B C \cdot C D,
$$

(Prop. VIII and IX.)

or

$$
c^{2}=a^{2}+b^{2}+2 a \cdot C D \text {. }
$$

Whence,

$$
C D=\frac{c^{2}-a^{2}-b^{2}}{2 a} \text {. }
$$

If $\angle C$ is acute, the segment $C D$ is negative as appears from the diagram.

Now

$$
\begin{aligned}
B D & =B C+C D \\
& =a+\frac{c^{2}-a^{2}-b^{2}}{2 a} \\
& =\frac{a^{2}+c^{2}-b^{2}}{2 a} .
\end{aligned}
$$

That is to say, whether $\angle C$ is acute or obtuse, the projection of the side $c$ upon the side $a$ equals $\frac{a^{2}+c^{2}-b^{2}}{2 a}$. 
Next, $\quad \overline{A D}^{2}=\overline{A B}^{2}-\overline{B D}^{2}$

(Prop. VII.)

$$
\begin{aligned}
& =(A B+B D)(A B-B D) \\
& =\left(c+\frac{a^{2}+c^{2}-b^{2}}{2 a}\right)\left(c-\frac{a^{2}+c^{2}-b^{2}}{2 a}\right) \\
& =\frac{(a+c)^{2}-b^{2}}{2 a} \cdot \frac{b^{2}-(a-c)^{2}}{2 a} \\
& =\frac{(a+b+c)(a-b+c)(a+b-c)(b+c-a)}{4 a^{2}},
\end{aligned}
$$

or

$$
A D=\frac{1}{2 a} \sqrt{(a+b+c)(a-b+c)(a+b-c)(b+c-a)} .
$$

This expression for the length of $A D$ can be written more conveniently if we let $a+b+c=2 s$,

so that

$$
\begin{aligned}
& a+b-c=2 s-2 c=2(s-c), \\
& a-b+c=2 s-2 b=2(s-b),
\end{aligned}
$$

and

$$
b+c-a=2 s-2 a=2(s-a) .
$$

Substituting these values in the expression for $A D$ gives

$$
\begin{aligned}
A D & =\frac{1}{2 a} \sqrt{2 s \cdot 2(s-a) \cdot 2(s-b) \cdot 2(s-c)} \\
& =\frac{2}{a} \sqrt{s(s-a)(s-b)(s-c)} .
\end{aligned}
$$

That is, the length of the perpendicular upon the side of a triangle from the opposite vertex $A$ equals $\frac{2}{a} \sqrt{s(s-a)(s-b)(s-c)}$.

Finally, the area of $\triangle A B C=\frac{1}{2} B C \cdot A D$,

$$
\begin{aligned}
& =\frac{1}{2} a \cdot \frac{2}{a} \sqrt{s(s-a)(s-b)(s-c)} \\
& =\sqrt{s(s-a)(s-b)(s-c) .}
\end{aligned}
$$




\section{EXERCISES}

1. What does the formula in Article 324 for the projection of the side $c$ upon the side $a$ become when $C$ is a right angle?

2. From symmetry write out the projections of (1) the side $c$ upon the side $b,(2)$ the side $a$ upon the side $b$, etc.

3. By symmetry, write out the expressions for the lengths of the perpendiculars from the vertices $B$ and $C$ upon the opposite sides.

4. Show that the shortest perpendicular falls upon the longest side of the triangle, and that any two perpendiculars are inversely proportional to the sides upon which they fall.

5. The three sides of a triangle are $58 \mathrm{ft}$., $51 \mathrm{ft}$., and $41 \mathrm{ft}$., respectively, find its area.

6. Find the lengths of the perpendiculars from the three vertices on the opposite sides of a triangle whose sides are 7 in., 9 in., and 11 in., respectively.

7. The sides $a, b, c$, of a triangle are 5, 9, 11 feet, respectively ; find whether or not it contains an obtuse angle, and the length of the projections of the sides $a$ and $b$ upon the side $c$.

8. Find the area in acres of a triangular field whose sides are 320 , 425, and 261 yards, respectively.

9. Find the sides of a right triangle if their projections upon the hypotenuse are $3 \frac{13}{1} \frac{3}{7}$ and $13 \frac{4}{17}$ feet, respectively.

10. The two parallel sides of a trapezoid are 16 and 21 feet, respectively, and their distance apart is 5 feet. Find the area.

11. The sum of the squares on the four sides of a parallelogram is equal to the sum of the squares on the diagonals.

12. The square on the base of an isosceles triangle is equal to twice the rectangle contained by either of the equal sides and the projection on it of the base.

13. If the square on one side of a triangle is less than the sum of the squares on the other two sides, how does the angle contained by these two sides compare with a right angle?

14. The base of a triangle being given, find the locus of the vertex when the sum of the squares on the two sides is also given.

Suggestion. Apply Ex. 1, p. 216. 


\section{SECTION III}

CONSTRUCTIONS

Proposition X

325. To construct a rectangle equal in area to a given parallelogram, and having one side equal to a given line-segment.
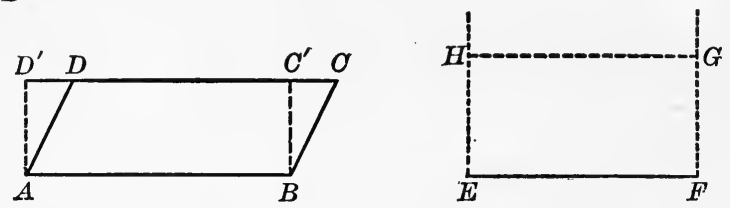

It is required to construct a rectangle upon the given linesegment $E F$ which shall be equal in area to the given parallelogram $A B C D$.

Construction. Draw $A D^{\prime}$ and $B C^{\prime}$ perpendicular to $C D$, thus forming a rectangle $A B C^{\prime} D^{\prime}$ equal in area to the given parallelogram $A B C D$.

(Art. 299.)

If we suppose $E F G H$ to be the required rectangle, of which the side $F G$ is as yet unknown,

then

$$
E F \cdot F G=A B \cdot B C^{\prime},
$$

since the area of a rectangle equals the product of two adjacent sides.

Therefore $E F: A \dot{B}=B C^{\prime}: F G$

(Art. 304.)

and the required side $F G$ can be found as the fourth proportional to $E F, A B$, and $B C^{\prime}$.

(Art. 272.)

The length of the side $F G$ having been thus found, the required rectangle is easily constructed as follows:-

At $E$ and $F$ draw straight lines perpendicular to $E F$.

Cut off $F G$ of the proper length, determined above, and make $E H$ equal to it. Join $H G$.

Prove that the figure as constructed is a rectangle. 
326. The method of analysis employed in the preceding construction (see page 86) should be resorted to whenever a direct solution of a problem does not readily appear.

It is strongly advised that the pupil actually make the constructions indicated in the exercises, drawing all lines carefully with ruler and compasses.

\section{EXERCISES}

1. Construct a parallelogram equal in area to a given triangle.

2. Construct a rectangle equal in area to a given triangle and having one side equal to a given line-segment.

3. Construct a square equal in area to a given parallelogram or triangle.

\section{Proposition XI}

327. To construct a square equal in area to the sum of two given squares.
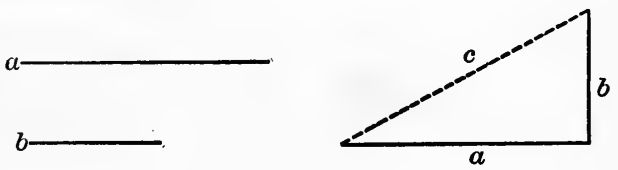

Let $a$ and $b$ be sides of the given squares. If they are placed at right angles, as in the diagram, then $c$ will equal a side of the required square. Why?

\section{EXERCISES}

1. Construct a square equal in area to the difference of two given squares.

2. Construct a square equal in area to the sum of two given triangles.

3. Construct a square which shall be equal to the sum of three given squares.

4. Construct a rectangle equal in area to a given square and having one side of given length. 


\section{Proposition XII}

328. To find two line-segments having the same ratio as the areas of two given squares.
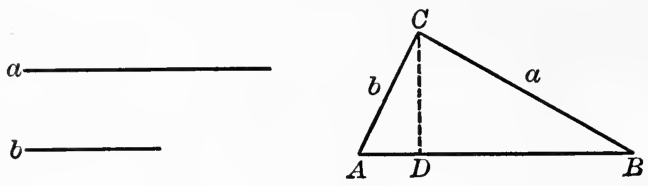

Let $a$ and $b$ be sides of the given squares.

Place these lines as in the diagram so as to form a right angle $A C B$ and join $A B$. The areas of the given squares are in the ratio $\overline{B C}^{2}: \overline{A C}^{2}$.

From $C$ draw $C D$ perpendicular to $A B$.

'Then

or

Also

or

$$
\begin{aligned}
A D: A C & =A C: A B \\
\overline{A C}^{2} & =A D \cdot A B .
\end{aligned}
$$$$
B D: B C=B C: B A \text {, }
$$$$
\overline{B C}^{2}=B D \cdot B A=D B \cdot A B .
$$

Hence

$$
\overline{B C}^{2}: \overline{A C}^{2}=D B \cdot A B: A D \cdot A B=D B: A D .
$$

Therefore $D B$ and $A D$ are the required line-segments.

\section{EXERCISES}

1. Construct a square which shall have a given ratio to a given square.

2. If $c$ is the third proportional to $a$ and $b$, show that $a$ is to $c$ as the square on $a$ is to the square on $b$.

3. Find two line-segments, one of given length, which shall be in the same ratio as the areas of two given squares.

4. Find two line-segments, one of given length, which shall be in the same ratio as the areas of two equilateral triangles. 


\section{Proposition XIII}

329. To construct a triangle equal in area to a given triangle and having one side of given length.

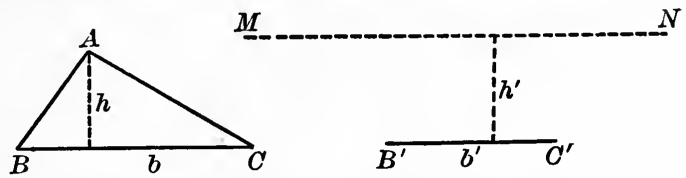

It is required to construct on the line-segment $B^{\prime} C^{\prime \prime}$ a triangle equal in area to a given triangle $A B C$.

Construction. Let $b$ and $h$ be the base and altitude, respectively, of the given triangle, and $b^{\prime}$ and $h^{\prime}$ the base and altitude of the required triangle, of which $h^{\prime}$ is unknown.

Then

$$
\frac{1}{2} h b=\frac{1}{2} h^{\prime} b^{\prime} \text {, or } h b=h^{\prime} b^{\prime} \text {. }
$$

Therefore

$$
b^{\prime}: b=h: h^{\prime} \text {; }
$$

and $h^{\prime}$ can be determined as the fourth proportional to $b^{\prime}$, $b$, and $h$.

(Art. 272.)

When $h^{\prime}$ has been so determined, draw a line $M N$ parallel to $B^{\prime} C^{\prime}$, whose distance from $B^{\prime} C^{\prime}$ equals $h^{\prime}$.

Then any triangle having $B^{\prime} C^{\prime}$ for base and its vertex in $M N$ will satisfy the given conditions.

\section{Proposition XIV}

330. To construct a triangle equal in area to the sum of two given triangles and having one side of given length.

1. Construct two triangles, $P$ and $Q$, equal in area to the given triangles, each having one side (the base) of the given length.

(Prop. XIII.)

2. Construct a triangle, $R$, having its base of the given length and its altitude equal to the sum of the altitudes of $P$ and $Q$.

The triangle $R$ fulfils the given conditions. Prove. 


\section{Proposition XV}

331. To reduce the number of sides of a polygon without altering the measure of its area.

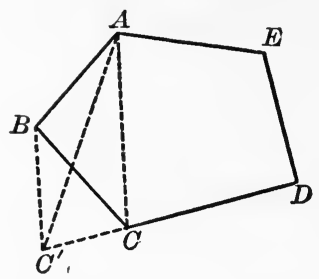

Let $A B C D E$ be the given polygon in which $A, B$, and $C$ are consecutive vertices.

Join $A C$ and through $B$ draw $B C^{\prime}$ parallel to $A C$, meeting the line $D C$ at $C^{\prime}$. Join $A C^{\prime}$.

$$
\triangle A C^{\prime \prime} C=\triangle A B C \text {. }
$$

Why?

Therefore the polygon $A C^{\prime} D E$ is equal in area to the original polygon $A B C D E$, and it has one less side.

By repeated applications of this process, the number of sides of any polygon can be reduced to three without altering the area.

\section{EXERCISES}

1. Apply the method of Proposition XV to reduce the number of sides of a polygon having a reëntrant angle, as in the diagram, the area to remain unaltered.

2. Construct a square equal in area to a given polygon.

First apply Proposition XV to reduce the polygon

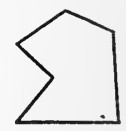
to a triangle.

3. Construct a polygon similar to a given polygon, $P$, and equal in area to another given polygon, $Q$.

Find squares equal in area to $P$ and $Q$. Let $m$ and $n$ be sides of these. Let $a$ be any side of $P$, and find $a^{\prime}$ a fourth proportional to $m, n$, and $a$. On $a^{\prime}$ construct a polygon, $R$, similar to $P, a$ and $a^{\prime}$ being homologous sides. $R$ is the required polygon. Prove. 


\section{Proposition XVI}

332. To construct a rectangle having given its area and the sum of two adjacent sides, or its perimeter.
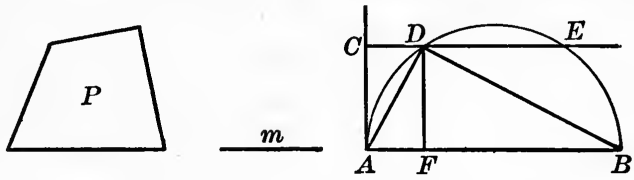

Let $P$ be equal to the given area and $A B$ the sum of two adjacent sides.

It is required to construct a rectangle having its area equal to $P$ and its perimeter equal to twice $A B$.

Construction. Find $m$ a side of the square whose area is equal to $P$.

On $A B$ describe a semicircle.

(Ex. 2, p. 224.)

At $A$ erect a perpendicular $A C$ equal to $m$, and through $C$ draw a straight line parallel to $A B$, cutting the semicircle at $D$ and $E$.

Draw $D F$ perpendicular to $A B$, and join $A D$ and $B D$.

$D F=m$, and $\overline{D F}^{2}=A F \cdot F B=m^{2}$.

(Art. 255.)

Therefore the rectangle whose adjacent sides are $A F$ and $F B$ is the one required.

333. In the above proposition suppose that $A B$ remains unaltered while $P$, the given area, is chosen larger. Then $m$, the side of the equivalent square, becomes longer and may be so long that the line drawn through $C$ parallel to $A B$ will not cut the semicircle. In that case, no rectangle can be constructed satisfying the conditions.

From a consideration of the conditions of this problem answers to the following questions will readily present themselves :

1. What is the greatest length of $m$ for which the required rectangle can be constructed?

2. What rectangle of given perimeter will have the greatest area?

3. What parallelogram with a given base and given perimeter will have the greatest area?

Such questions naturally lead to a further consideration of what we have called maximum and minimum values. (See Art. 175.) 


\section{Section IV}

\section{MAXIMA AND MINIMA}

334. When a geometrical figure varies continuously it sometimes happens that one or more of the variable magnitudes connected with it gradually increases for a time and afterward begins to decrease. When such a magnitude has reached its greatest value, that is, just when it ceases to increase and begins to decrease, it is said to have a maximum value. On the other hand, if the magnitude is first decreasing and then begins to increase, at the time the change takes place the magnitude is said to have a minimum value.

Suppose we recall the example given in Article 175.

Take within a circle any point $P$, not the centre, and join it to a point $A$ on the circle. Let $A$ move continuously along the circle in the way indicated by the arrowhead. The straight line $P A$ will steadily increase in length till it reaches that position in which it passes through the centre; after that it will steadily decrease for a time.

So we say, the line $P A$ is a maximum when it passes through the centre.

As the rotation continues, the line $P A$ will reach

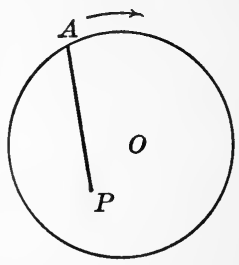
a minimum in that position where, if produced backward, it would pass through the centre.

Since a maximum or minimum value of a variable magnitude occurs at a critical position which may be approached from either side, we naturally look for a maximum or a minimum when the variable magnitude is symmetrically situated with respect to the rest of the figure, and this usually proves to be the case.

Thus, the shortest straight line from a given point to a straight line not passing through it is the perpendicular to the line. 


\section{Examples of Maxima and Minima}

1. If two sides of a triangle are of given lengths while the third side varies, the triangle will have the maximum area when the first two sides are at right angles.

Let $A B$ and $A C$ be given sides of a triangle $A B C$, and let the angle between them vary continuously, so that, while $A B$ remains fixed, the side $A C$ rotates about $A$, taking up successively the positions $A C_{1}, A C_{2}, A C_{3}, \ldots$

The area of $\triangle A B C$ equals one-

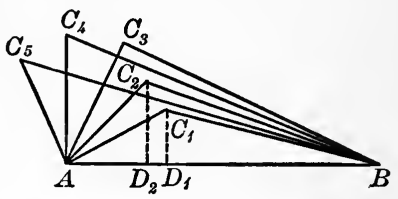
half the product of $A B$ and the perpendicular from $C$ upon $A B$. The perpendicular $C D$ steadily increases with the rotation until $A C$ makes right angles with $A B$, after which it begins to decrease.

The area of $\triangle A B C$ therefore gradually increases with the rotation till $A C$ is perpendicular to $A B$, after which it decreases.

Hence the area is a maximum when $A C$ and $A B$ are at right angles.

2. In a straight line, to find a point such that the sum of its distances from two given points on the same side of the line shall be a minimum.

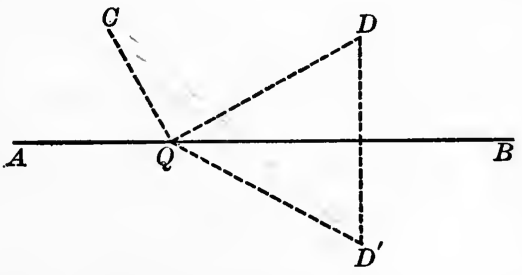

The problem is to find a point $Q$ in $A B$ such that $C Q+Q D$ has a minimum value.

Let $D^{\prime}$ be the inverse point (Art. 144) of $D$ with respect to the line $A B$. Take any point $Q$ in $A B$. Then $Q D^{\prime}=Q D$. Why? 
Therefore $C Q+Q D^{\prime}=C Q+Q D$.

Hence $C Q+Q D$ is least when $C Q+Q D^{\prime}$ is least.

What then is the required position of $Q$ ?

3. Given two intersecting straight lines $A B, A C$, and a point $P$ between them; of all line-segments which pass through $P$ and are terminated by $A B$ and $A C$, that which is bisected at $P$ makes with $A B$ and $A C$ the triangle of minimum area.

Let the line-segment $D E$ be bisected at $P$. It is required to show that $\triangle D A E$ is less than $\triangle F A G$, where $F G$ is any other line-segment through $P$.

Through $D$ draw $D M$ parallel to $A C$.

Then $\triangle P D M$ is identically equal to $\triangle P E G$. Why?

But $\triangle P D M$ is less than $\triangle P D F$. Therefore $\triangle P E G$ is less than $\triangle P D F$.

To each add the figure $A D P G$. Therefore $\triangle D A E$ is less than

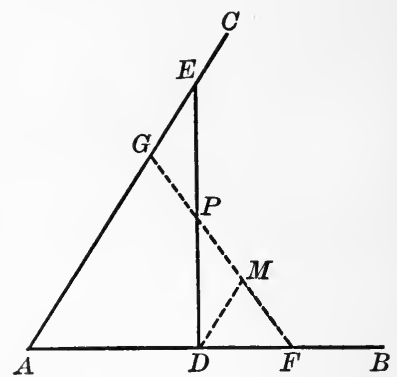
$\triangle F A G$.

\section{EXERCISES}

1. What is the maximum chord of a circle? What is the minimum linesegment from a given point outside to a given circle?

2. Of all triangles having the same base and equal areas, that which is isosceles has the minimum perimeter.

3. Of all triangles having the same area, that which is equilateral has the minimum perimeter.

4. Of all triangles having the same base and equal perimeters, that which is isosceles has the maximum area.

Definition. Figures having equal perimeters are called isoperimetric.

5. Given the base and the vertical angle of a triangle, construct the triangle so that its area may be a maximum.

6. Divide a given line-segment into two parts so that the sum of the squares on the parts may be a minimum.

7. Divide a given line-segment into two parts so that the rectangle contained by the parts is a maximum. 


\section{MISCELLANEOUS EXERCISES}

1. Construct a parallelogram equal in area to a given parallelogram, and having one of its angles equal to a given angle.

2. $A B C$ is a given triangle ; construct a triangle of equal area having $A B$ for base and its vertex in a given straight line.

3. $A B C D$ is a parallelogram ; from any point $P$ in the diagonal $B D$, the straight lines $P A, P C$ are drawn. Show that the triangles $P A B$ and $P C B$ are equal in area.

4. The sides $A B, A C$ of a triangle are bisected at $C^{\prime} B^{\prime}$, respectively ; $C C^{\prime}, B B^{\prime}$ intersect at $F$. Prove that the triangle $B F C$ is equal to the quadrilateral $A C^{\prime} F B^{\prime}$.

5. The locus of a point such that the sum of the squares on its distances from two fixed points is equal to the square on the distance between the points is a circle passing through the two points.

6. The locus of a point such that the difference of the squares on its distances from two fixed points is equal to the square on the distance between the points is a straight line.

7. If $A B C D$ is a square and the vertex $A$ is joined to the mid-point of the side $B C, B$ to the mid-point of $C D, C$ to the mid-point of $D A$, and $D$ to the mid-point of $A B$, the lines so drawn are the sides of a square whose area is one-fifth of the area of the original square.

8. Construct two line-segments in the ratio $1: \sqrt{2}$.

9. Divide a given triangle into two equal parts by a straight line parallel to one of its sides.

10. Construct two line-segments in the ratio $1: \sqrt{3}$.

11. Draw a straight line-parallel to one side of a given triangle so as to form a triangle equal to one-third of the original triangle.

12. If three line-segments are in continued proportion, the first is to the third as the area of a triangle described on the first is to the area of the similar triangle described on the second.

13. If any point within a parallelogram is joined to the four vertices, the sum of either pair of triangles having parallel bases is half the area of the parallelogram.

14. The sides of a triangle are $21,24,35$ feet, respectively. Find the area of the triangle and the projection of the lesser side upon each of the others. 
1. Definitions.

\section{SUMMARY OF CHAPTER IV}

(1) Adjacent Polygons - polygons having one or more sides or parts of sides in common. $\$ 288$.

(2) Sum of Two Polygons - a polygon formed by making the two adjacent and disregarding the common boundary. $\$ 288$.

(3) Area of a Plane Closed Figure - surface enclosed by the figure, or the measure of that surface. $\$ \S 289,291$.

(4) Altitude of a Parallelogram - the distance between the base and the opposite side. $\$ 298$.

(5) Altitude of a Triangle-the distance between the base and the opposite vertex. $\$ 298$.

(6) Projection of a Point on a Line - the foot of the perpendicular drawn from the point to the line. $\$ 319$.

(7) Projection of a Given Line-segment on Any Straight Line - the intercept between the projections of the extremities of the segment on the line. $\S 319$.

(8) Isoperimetric Figures - those having equal perimeters. Ex. 4, p. 228.

2. Axions.

(1) If two plane polygons or other closed figures are identically equal, their areas are equal (Axiom 11). $\$ 289$.

(2) The sum of the areas of two plane polygons is equal to the area of their sum (Axiom 12). $\$ 289$.

3. Problems.

(1) To construct a rectangle equal in area to a given parallelogram, and having one side equal to a given line-segment. $\$ 325$.

(2) To construct a square equal in area to the sum of two given squares. $\$ 327$.

(3) To find two line-segments having the same ratio as the areas of two given squares. $\S 328$.

(4) To construct a triangle equal in area to a given triangle and having one side of given length. $\S 329$.

(5) To construct a triangle equal in area to the sum of two given triangles and having one side of given length. $\$ 330$.

(6) To reduce the number of sides of a polygon without altering the measure of its area. $\S 331$.

(7) To construct a rectangle having given its area and the sum of two adjacent sides, or its perimeter. $\S 332$. 
(8) In a straight line to find a point such that the sum of its distances from two given points on the same side of the line shall be a minimum. $\S 334.2$.

4. Theorems on the Areas of Parallelograms.

(1) Parallelograms upon the same base and between the same parallels are equal in area. $\$ 292$.

(2) Parallelograms upon equal bases and between the same parallels are equal in area. $\$ 294$.

(3) Any parallelogram is equal in area to a rectangle having an equal base and an equal altitude. $\S 299$.

(4) The areas of two rectangles having equal altitudes are in the same ratio as their bases. $\S 300$.

(5) The areas of any two rectangles are in the same ratio as the products of their bases and altitudes. $\S 303$.

(6) The area of any rectangle is equal to the product of its base and its altitude. $\$ 304$.

(7) The area of a parallelogram is equal to the product of its base and its altitude. $\S 305$.

(8) The area of a square is equal to the square of any one of its sides. $§ 307$.

(9) The areas of two parallelograms having an angle of the one equal to an angle of the other are in the same ratio as the products of the sides containing the equal angles. $\S 309$.

\section{Theorems on the Areas of Triangles.}

(1) If a triangle and a parallelogram are upon the same base, or upon equal bases, and between the same parallels, the area of the triangle equals half the area of the parallelogram. $\$ 295$.

(2) Triangles upon the same base, or upon equal bases, and between the same parallels, are equal in area. $\$ 296$.

(3) Triangles upon equal bases in the same straight line, having their opposite vertices in common, are equal in area. $\$ 297$.

(4) The areas of two triangles having equal altitudes are in the same ratio as their bases; or having equal bases are in the same ratio as their altitudes. $\S 302$.

(5) The area of a triangle is equal to half the product of its base and altitude. $\$ 306$.

(6) The areas of two triangles having an angle of the one equal to an angle of the other are in the same ratio as the products of the sides containing the equal angles. $\S 308$.

(7) Area of a triangle in terms of the sides. See $\S 324$. 


\section{Theorems on the Areas of Similar Polygons.}

(1) The areas of similar triangles are in the same ratio as the squares of any two homologous sides. $\$ 314$.

(2) The areas of similar polygons are in the same ratio as the squares of any two homologous sides. $§ 315$.

(3) The areas of similar polygons are in the same ratio as the squares of any two homologous diagonals. $\$ 316$.

(4) The area of the square described on the hypotenuse of a right triangle is equal to the sum of the areas of the squares described on the other two sides. $\$ 317$.

(5) The area of any polygon described on the hypotenuse of a right triangle is equal to the sum of the areas of the similar polygons similarly described on the other two sides. $§ 318$.

\section{Miscellaneous Theorems.}

(1) If a given line-segment is divided internally into any two parts, the square on the whole segment is equal in area to the sum of the squares on the two parts together with twice the rectangle contained by the two parts. $\S 311$.

(2) If a given line-segment is divided externally into any two parts, the square on the given segment is equal in area to the sum of the squares on the two parts less twice the rectangle contained by the two parts. $\S 312$.

(3) In any triangle the square on the side opposite an acute angle is less than the sum of the squares on the other two sides by twice the product of either of these sides and the projection of the other upon it. $\$ 321$.

(4) In an obtuse-angled triangle the square on the side opposite the obtuse angle is greater than the sum of the squares on the other two sides by twice the product of either of these sides and the projection of the other upon it. $\$ 322$.

(5) If two sides of a triangle are of given lengths while the third side varies, the triangle will have the maximum area when the first two sides are at right angles. $\S 334.1$.

(6) Given two intersecting straight lines $A B, A C$, and a point $P$ between them; of all line-segments which pass through $P$ and are terminated by $A B$ and $A C$, that which is bisected at $P$ makes with $A B$ and $A C$ the triangle of minimum area. $\S 334.3$. 


\title{
CHAPTER V
}

\section{MEASUREMENT OF THE CIRCLE}

\author{
$\rightarrow \infty 05000$ \\ Section I \\ REGULAR POLYGONS
}

Definitions

335. A regular polygon is a polygon which is both equilateral and equiangular.

For example, an equilateral triangle is a regular polygon of three sides; a square is a regular polygon of four sides.

A polygon of more than three sides may be equilateral without being equiangular, or it may be equiangular without being equilateral; but in order to be classed as regular it must be both equilateral and equiangular.

That there can be regular polygons of any given number of sides will be seen from the first proposition of this chapter.

Make a diagram of a quadrilateral that is (1) equilateral, but not equiangular; (2) equiangular, but not equilateral ; (3) both equilateral and equiangular; (4) neither equilateral nor equiangular.

336. A regular polygon of more than four sides may or may not be convex, but unless the contrary is stated, it will be understood that any figure under discussion is convex.

A polygon of five sides is called a pentagon; one of six sides, a hexagon; one of seven sides, a heptagon ; one of eight sides, an octagon; one of ten sides, a decagon; one of twelve sides, a dodecagon. 
337. Postulate 8. A circle may be divided into any given number of equal arcs.

The problem "to divide a circle, or any arc of a circle, into a given number of equal parts" is not always solvable by the methods of elementary geometry. The method of solution, if there is one, must of course depend on the number of such parts required. All that the above postulate affirms is that the circle may be thought of as made up of any specified number of equal parts, and the points of division may be assumed. It says nothing at all about a method of finding these points of division.

338. In the preceding chapters we have shown how to divide line-segments, angles, and arcs of circles, into certain numbers of equal parts. The following exercises will recall the methods employed.

\section{EXERCISES}

1. Divide a given line-segment into two equal parts; into four equal parts ; into eight equal parts (Art. 56).

2. Divide a given line-segment into three equal parts ; into six equal parts ; into nine equal parts (Art. 142).

3. Divide a given line-segment into any required number of equal parts (Art. 269).

4. Divide a given angle into two, four, eight, etc. equal parts (Art. 54).

5. Divide a right angle into three equal parts (Art. 142).

6. Divide a given are of a circle into two, four, eight, etc., equal parts (Art. 165).

The problem to divide any given angle or arc of a circle into three equal parts has been found to be impossible of solution by the methods of elementary geometry, in which we make use of the ruler and compasses only.

7. Divide a circle into two, four, eight, etc., equal arcs.

8. Divide a circle into three, six, or twelve equal arcs. (Make use of Ex. 5.) 


\section{Proposition I}

339. If a circle is divided into any number of equal arcs:

I. The chords joining the points of division, taken in order, form a regular inscribed polygon.

II. The tangents to the circle at the points of division, taken in order, form a regular circumscribed polygon.

REMARK. The number of sides of the polygon is equal in each case to the number of points of division in the circle.

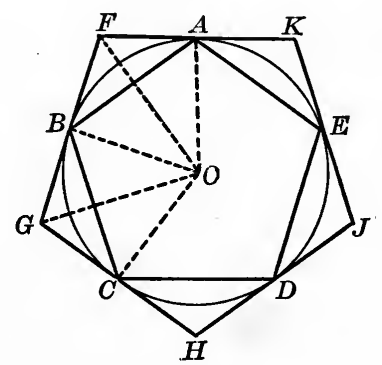

Let a given circle be divided into any number of equal arcs $A B, B C, C D$, etc., and let the points of division be joined in order, thus forming the polygon $A B C D E$; also at the points of division, let the tangents to the circle be drawn, thus forming the polygon $F G H J K$.

It is required to prove that the polygon $A B C D E$ is a regular polygon; and also, that the polygon FGHJK is a regular polygon.

Proof. First, since by hypothesis the arcs $A B, B C, C D$, etc., are equal, the chords $A B, B C, C D$, etc., are equal. (Art. 161.)

Therefore the polygon $A B C D E$ is equilateral.

Also, the arc $E A B=$ the arc $A B C$. Why?

Therefore, $\angle E A B=\angle A B C$. 
Similarly $\angle B C D=\angle A B C$, and so on.

Therefore the polygon $A B C D E$ is equiangular.

That is, the polygon $A B C D E$ being both equilateral and equiangular is regular.

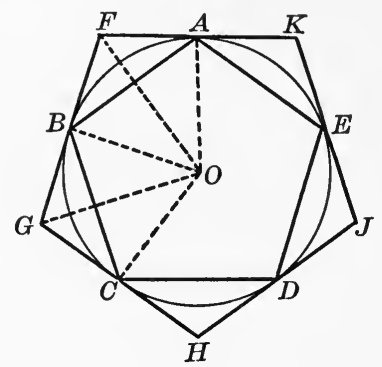

Next, let the points $A, B, C$, etc., be joined to the centre $O$. Then $\angle \mathrm{s} A O B, B O C, C O D$, etc., are all equal. Why ?

Therefore, $\angle \mathrm{s} A F B, B G C, C H D$, being supplements of equal angles at the centre [why?] are also equal.

Hence the polygon $F G H J K$ is equiangular.

Also, the line-segment $A F=$ the line-segment $F B$. (Art. 194.) Also, the line-segment $F B=$ the line-segment $B G$. Prove. Similarly $B G=G C, G C=C H$, and so on.

Therefore the side $F G=$ the side $G H,=$ the side $H J$, and so on.

Hence the polygon $F G H J K$ is equilateral.

That is, the polygon $F G H J K$ is also regular.

340. Corollary I. If the vertices of a regular inscribed polygon are joined to the mid-points of the arcs subtended by the sides of the polygon, the joining lines will form another regular. polygon of twice the number of sides.

341. Corollary II. If tangents are drawn at the mid-points of the arcs between the points of contact of the sides of a regular circumscribed polygon, these together with the sides of the original polygon form a regular polygon of twice the number of sides. 


\section{Proposition II}

342. Any equilateral polygon inscribed in a circle is also equiangular, and hence regular.

For the equal sides of the polygon subtend equal ares of the circle (Art. 163), these arcs together making up the whole circle (or some multiple of the circle, if the polygon is not convex). The angles between consecutive sides of the polygon also subtend equal arcs, and are therefore equal. Hence the polygon is regular.

Is the converse of this theorem true?

\section{EXERCISES}

1. If a circle is divided into five equal arcs at the points $A, B, C, D, E$, and the points are joined, each to the next but one, as in the diagram, prove that the resulting figure is a regular polygon, according to the definition, though not a convex regular polygon.

Suppose the circle is divided into seven equal arcs, construct an inscribed polygon by joining each vertex to the next but one, next but two, next but three, etc. Show that the polygons so constructed are regular.

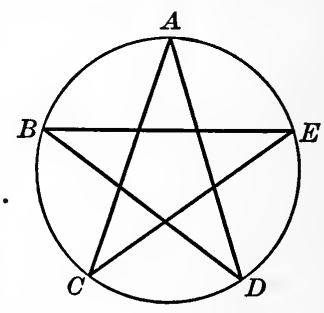

2. All regular convex polygons of the same number of sides inscribed in the same circle are equal; and all regular convex polygons of the same number of sides circumscribed about the same circle are equal.

3. The perimeter of a regular polygon inscribed in a circle is less than the perimeter of a circumscribed polygon of the same number of sides.

4. The perimeter of a regular polygon inscribed in a circle is less than an inscribed polygon of twice the number of sides.

5. Any parallelogram inscribed in a circle is rectangular.

6. If a rectangle is circumscribed about a circle it must be a square.

7. What is the measure of the angle at the centre of a circle subtended by the side of a circumscribed square? 


\section{Proposition III}

343. A circle can be circumscribed about any regular polygon, and another circle can be inscribed in it.

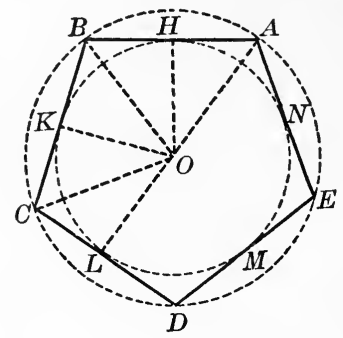

Let $A B C D E \ldots$ be any regular polygon.

It is required to prove that a circle can be circumscribed about it, and that another circle can be inscribed in it.

Proof. First, at the mid-points $H$ and $K$ of two adjacent sides, $A B$ and $B C$, of the given polygon, draw the perpendiculars to these sides, and let them meet at the point $O$.

Then $O$ is the centre of the circle which can be made to pass through the three vertices $A, B$, and $\mathrm{C}$.

(Art. 151.)

Since $O A=O B=O C$, and $A B=B C, \triangle \mathrm{s} O A B$ and $O B C$ are equal isosceles triangles, and therefore

$$
\angle O A B=\angle O B A=\angle O B C=\angle O C B .
$$

That is, $\angle O B C$ is one half of $\angle A B C$.

Therefore $\angle O C B$ is one half of the equal angle $B C D$,

or

$$
\angle O C B=\angle O C D \text {. }
$$

Join $O D$.

Then $\triangle \mathrm{s} C B O$ and $C D O$ are identically equal.

Therefore

$$
O D=O B,
$$

$$
\angle O D C=\angle O B C=\frac{1}{2} \angle C D E \text {. }
$$


Similarly, $O E$ may be shown to be equal to $O C$, and so on for other vertices.

Therefore the circle which passes through the vertices $A, B$, and $C$ will also pass through the remaining vertices $D, E$, etc.

Hence, a circle can be circumscribed about the given polygon.

Next, since $\triangle \mathrm{s} A O B, B O C, C O D$, etc., are equal isosceles triangles, their altitudes $O H, O K, O L, O M$, and $O N$ are all equal. Prove.

Therefore the circle described with centre $O$, and radius $O H$, will pass through the points $K, L, M, N$, and the sides of the polygon will be tangent to the circle, since each side is at right angles to a radius at its extremity.

(Art. 187.)

Therefore a circle can be inscribed in the given polygon.

\section{Definitions}

344. The common centre of the inscribed and circumscribed circles of a regular polygon is called the centre of the polygon.

345. The radius of the circumscribed circle, or the linesegment joining the centre and a vertex of a regular polygon, is called the radius of the polygon.

346. The radius of the inscribed circle, or the perpendicular from the centre on a side of a regular polygon, is called the apothem of the polygon.

347. Corollary I. Any radius of a regular polygon bisects the angle at the vertex.

348. Corollary II. The angle formed by two consecutive radii of a regular polygon equals four right angles divided by the number of sides of the polygon.

This angle is sometimes spoken of as 'the angle at the centre of the polygon.' 


\section{Proposition IV}

349. Any two regular polygons of the same number of sides are similar.
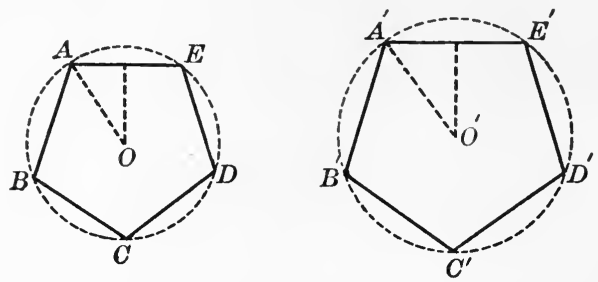

Let $A B C D E$ and $A^{\prime} B^{\prime} C^{\prime} D^{\prime} E^{\prime}$ be two regular polygons of the same number of sides.

It is required to prove that they are similar.

Proof. First, the two polygons are mutually equiangular, since in each polygon the interior angles are all equal, and the magnitude of an interior angle of either polygon depends only on the number of sides, which is the same in both.

Next, the homologous sides are proportional.

For

$$
A B=B C=C D=\text { etc. }
$$

and

$$
A^{\prime} B^{\prime}=B^{\prime} C^{\prime}=C^{\prime} D^{\prime}=\text { etc. }
$$

Therefore $A B: A^{\prime} B^{\prime}=B C: B^{\prime} C^{\prime}=C D: C^{\prime} D^{\prime}=$ etc.

Therefore the two polygons are similar.

350. Corollary I. Homologous sides in two regular polygons of the same number of sides are in the same ratio as the radii of the circumscribed circles, or as the radii of the inscribed circles; that is, as the radii of the polygons, or as the apothems of the polygons.

Suggestion. Draw the radii and apothems and so obtain similar triangles in the two polygons. 
351. Corollary II. The perimeters of two regular polygons of the same number of sides are in the same ratio as their radii, or as their apothems.

Let $S_{1}$ and $S_{2}$ be the lengths of the sides in two regular polygons of $n$ sides, of which $A_{1}$ and $A_{2}$ are the apothems. Then $S_{1}: S_{2}=A_{1}: A_{2}$ (Cor. I). Therefore $n S_{1}: n S_{2}=A_{1}: A_{2}$, that is, the perimeters are in the same ratio as the apothems. The same holds true for the radii.

352. Corollary III. The areas of two regular polygons of the same number of sides are in the same ratio as the squares of their radii, or as the squares of their apothems.

(Art. 315.)

\section{EXERCISES}

1. Tangents to a circle at the mid-points of the arcs subtended by the sides of a regular inscribed polygon form a regular circumscribed polygon.

The sides of the circumscribed polygon are parallel to the sides of the inscribed polygon (Art. 192), and the vertices of the circumscribed lie upon the radii, produced, of the inscribed.

Suggestion. To show that the vertex $P$ lies on the radius $O A$, show by similar triangles that the tangent at $H$ cuts $O A$ in the same ratio as does the tangent at $K$.

Are these two polygons similar?

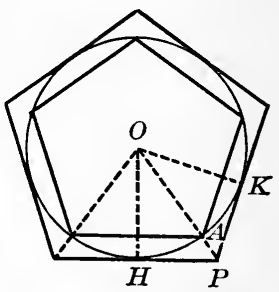

2. The interior angle of a regular polygon of $n$ sides equals $\frac{2}{n}(n-2)$ right angles. (Art. 118.)

3. The interior angle of any regular polygon is the supplement of the angle at the centre of the polygon; that is, it is equal to $180^{\circ}-\frac{360}{n}$.

Show that this statement agrees with the statement of Ex. 2.

4. Prove that the side of a regular hexagon is equal to a radius of the circumscribed circle.

5. Find in degrees the angle (1) of a regular pentagon, (2) of a regular hexagon, (3) of a regular octagon.

6. Regular pentagons are inscribed in two circles of five and eight feet radius, respectively. Find the ratio of their perimeters and of their areas. 


\section{Proposition V}

353. The area of a regular polygon is equal to half the product of its perimeter and its apotherm.

Proof. If all the vertices of the polygon are joined to the centre, the triangles so formed are all equal, and the area of each equals half the product of a side and the apothem.

(Art. 306.)

Therefore the area of the whole polygon equals half the product of its perimeter and its apothem.

\section{Proposition VI}

354. If the number of sides of a regular inscribed polygon be doubled the perimeter will be increased, but if the number of sides of a regular circumscribed polygon be doubled the perimeter will be diminished.

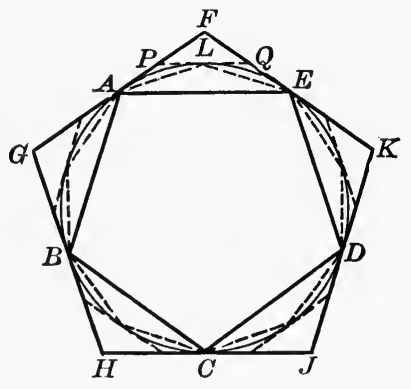

Let $A B C D E$ be any regular polygon inscribed in a circle, and FGHJK a regular polygon circumscribed about the same circle.

For convenience, we may let the two polygons have the same number of sides and be so arranged that the vertices of the inscribed coincide with the points of contact of the circumscribed. 
It is required to prove that if the number of sides of the inscribed polygon be doubled, say by joining the mid-points of the arcs of the circle to the vertices, the perimeter will be increased; but if the number of sides of the circumscribed polygon be doubled, say by drawing tangents at the mid-points of the arcs of the circle, the perimeter will be diminished.

Proof. Let $L$ be the mid-point of the arc $A E$, and $P Q$ be tangent to the circle at $L$.

First, in $\triangle A L E$, the sum of $A L$ and $L E$ is greater than $A E$.

(Art. 70.)

Therefore the perimeter of the regular polygon of which $A L$ and $L E$ are two adjacent sides is greater than the perimeter of the polygon of which $A E$ is a side.

Next, in $\triangle F P Q$, the side $P Q$ is less than the sum of $P F$ and $F Q$.

Therefore the sum of $A P, P Q$, and $Q E$ is less than the sum of $A F$ and $F E$.

Hence the perimeter of the regular polygon of which $P Q$ is one side is less than the perimeter of the given circumscribed polygon.

\section{Proposition VII}

355. The area of a regular inscribed polygon is increased, and the area of a regular circumscribed polygon is diminished, when the number of sides is doubled.

Proof. The area of a regular polygon equals half the product of its perimeter and its apothem.

(Prop. V.)

When the number of sides of a regular inscribed polygon is doubled, the perimeter is increased (Prop. VI), as is also the apothem [why?]. Hence the area is increased.

When the number of sides of a regular circumscribed polygon is doubled, the perimeter is diminished (Prop. VI), while the apothem remains unaltered. Hence the area is diminished. 


\section{SeCtion II}

\section{MEASUREMENT OF THE CIRCLE}

356. In the preceding chapters, whenever we have spoken of a length, we have had in mind a straight line distance; it has been in every case the measure of a line-segment.

What is meant by the length of a curved line is not so evident, and hence it is necessary to give this expression a meaning by definition.

357. If any regular polygon is inscribed in a given circle, and the number of its sides is repeatedly doubled (or is indefinitely increased in any regular way), the polygon can be made as nearly as you please to coincide with the circle.

In other words, the circle is the limit which the regular inscribed polygon approaches, as the number of its sides is indefinitely increased.

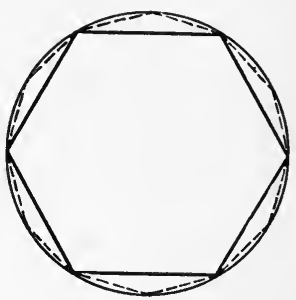

The apothem of the inscribed polygon approaches the radius of the circle as its limit.

358. Definition. The length of a circle is defined to be the limit of the perimeter of an inscribed regular polygon, as the number of sides of the polygon is indefinitely increased.

The fact that the perimeter of a variable inscribed regular polygon has a limit admits of a formal proof, the essential points of which are: (1) the series of perimeters is constantly increasing (Prop. VI); (2) the perimeter never exceeds a fixed finite quantity, for example, the perimeter of a particular circumscribed polygon.

The length of a circle is called its circumference.

When the number of sides of an inscribed regular polygon is indefinitely increased, the 'circle' is the limit of the 'polygon'; the 'circumference of the circle' is the limit of the 'perimeter of the polygon.' 
The length of an arc of a circle is defined in the same way to be the limit of the sum of chords in the arc when the number of such chords is indefinitely increased in some regular way.

359. Dewinition. The area of a circle is the surface enclosed by the circle. It is equal to the limit of the area of a regular inscribed polygon as the number of its sides is indefinitely increased.

360. The circumference of a circle could just as well be defined as the limit of the perimeter of a regular circumscribed polygon when the number of its sides is indefinitely in-

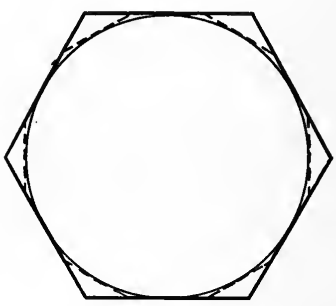
creased, since the limit of the perimeter of the circumscribed polygon is the same as the limit of the perimeter of the inscribed polygon.

This statement again admits of a formal proof, which however involves a greater knowledge of algebra than the pupil is supposed to have at this stage. The assertions that the circle is the limit of the regular inscribed polygon (Art. 357), and also of the regular circumscribed polygon (Art. 360 ) may be taken as postulates.

The radius of the circumscribed polygon approaches the radius of the circle as its limit, and the area of the polygon the area of the circle as its limit.

361. Since the perimeter of the inscribed polygon continually increases and the perimeter of the circumscribed polygon continually decreases as the number of their sides is indefinitely increased (Prop. VI) :

1. The circumference of a circle is greater than the perimeter of any regular polygon inscribed in it.

2. The circumference of a circle is less than the perimeter of any regular polygon circumscribed about it. 


\section{Proposition VIII}

362. The ratio of the circumference of a circle to its diameter is the same for all circles.
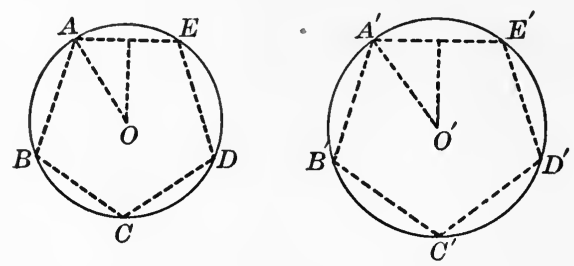

Let $O$ and $O^{\prime}$ be the centres of any two circles whose circumferences are denoted by $C$ and $C^{\prime}$, their diameters by $d$ and $d^{\prime}$, and their radii by $r$ and $r^{\prime}$.

It is required to prove that

$$
C: d=C^{\prime \prime}: d^{\prime} .
$$

Proof. Inscribe in the two given circles regular polygons $A B C D E$ and $A^{\prime} B^{\prime} C^{\prime \prime} D^{\prime} E^{\prime}$ of the same number of sides. The perimeters of these polygons are in the same ratio as their radii, i.e. as the radii of the circles in which they are inscribed.

Let $P$ and $P^{\prime}$ be the perimeters of the two polygons.

(Prop. IV, Cor. II.)

Then

or

$$
\begin{aligned}
P: P^{\prime} & =r: r^{\prime}, \\
\frac{P}{r} & =\frac{P^{\prime}}{r^{\prime}} .
\end{aligned}
$$

Suppose now the number of sides in each polygon is doubled, and let the perimeters of the polygons so formed be denoted by $P_{1}$ and $P_{1}^{\prime}$.

Then

$$
\frac{P_{1}}{r}=\frac{P_{1}^{\prime}}{r^{\prime}} \text {. }
$$

If this process is repeated indefinitely, the perimeter $P$ will approach the circumference $C$ as its limit, and the perimeter $P^{\prime}$ will approach the circumference $C^{\prime}$ as its limit. (Art. 357.) 
Therefore

$$
\frac{C}{r}=\frac{C^{\prime}}{r^{\prime}} .
$$

Also

$$
\frac{C}{2 r}=\frac{C^{\prime}}{2 r^{\prime}}
$$

or

$$
\frac{C}{d}=\frac{C^{\prime}}{d^{\prime}}
$$

Since the two given circles are any circles whatsoever, the ratio of the circumference to the diameter in any one circle is equal to the ratio of the circumference to the diameter in any other circle.

This ratio is denoted by the symbol $\pi$ (called Pī), so that for all circles

or

$$
\frac{C}{d}=\pi,
$$

$$
C=\pi d=2 \pi r \text {. }
$$

363. It should be carefully noted that the symbol $\pi$ represents a fixed and definite number, whose relation to other numbers however we have not yet determined. The old problem of 'squaring the circle,' upon which so much time and labor were expended, was "to find the side of a square whose area is equal to the area of a given circle," and this involved finding the value of $\pi$ in terms of ordinary numbers. It was shown in 1882 , by Lindemann, a German mathematician, that $\pi$ cannot be so expressed. We can, however, approximate its value in ordinary numbers, and this will be done in a subsequent proposition.

\section{EXERCISES}

1. Show that the area of an inscribed square equals $2 r^{2}$.

2. Show that the area of an inscribed regular hexagon equals $3 r^{2} \frac{\sqrt{3}}{2}$, and that the distance of any side from the centre is $r \frac{\sqrt{3}}{2}$.

3. The ratio of the circumference of a circle to the perimeter of an inscribed square is $\pi: 2 \sqrt{2}$, and to the perimeter of an inscribed hexagon is $\pi: 3$. 


\section{Proposition IX}

364. The area of a circle is equal to one-half the product of its circumference and radius.

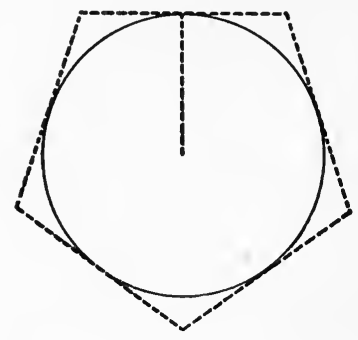

Let $A$ be the area of the given circle, $r$ its radius, and $C$ its circumference.

It is required to prove that $A=\frac{1}{2} C r$.

Proof. Circumscribe a regular polygon about the given circle, and denote its perimeter by $P$, and its area by $H$.

Then since the apothem of this polygon is $r$,

$$
H=\frac{1}{2} \operatorname{Pr} \text {. }
$$

If now the number of sides of the polygon be doubled, and the perimeter of this new polygon be denoted by $P_{1}$ and its area by $H_{1}$, then again

$$
H_{1}=\frac{1}{2} P_{1} r
$$

If this process is repeated indefinitely, the perimeter $P$ approaches the circumference $C$ as its limit, $\frac{1}{2} P r$ approaches $\frac{1}{2} C r$ as its limit, and the area $H$ approaches the area of the circle $A$ as its limit.

(Art. 360.)

Since the variable $H$ always equals the variable $\frac{1}{2} \operatorname{Pr}$, the limit $A$ equals the limit $\frac{1}{2} C r$.

(Art. 230.)

Or

$$
A=\frac{1}{2} C r \text {. }
$$


365. Corollary I. The area of a circle is equal to $\pi$ times the square of its radius, or $\frac{\pi}{4}$ times the square of its diameter.

For since

$$
\begin{aligned}
& C=2 \pi r=\pi d, \\
& A=\pi r^{2}=\frac{1}{4} \pi d^{2},
\end{aligned}
$$

where $d$ is the diameter of the circle.

366. Corollary II. The areas of two circles are in the same ratio as the squares of their radii, or as the squares of their diameters.

If $A$ is the area of a circle whose radius is $r$ and $A^{\prime}$ is the area of a circle whose radius is $r^{\prime}$,

then

$$
\frac{A}{A^{\prime}}=\frac{\pi r^{2}}{\pi r^{\prime 2}}=\frac{r^{2}}{r^{\prime 2}}
$$

and

$$
\frac{A}{A^{\prime}}=\frac{\frac{1}{4} \pi d^{2}}{\frac{1}{4} \pi d^{\prime 2}}=\frac{d^{2}}{d^{\prime 2}}
$$

\section{Definitions}

367. Similar arcs of circles are ares which subtend equal angles at the centres of the circles of which they are parts.

Thus $A B$ and $A^{\prime} B^{\prime}$ are similar arcs of the circles $O$ and $O^{\prime}$, if the angles at
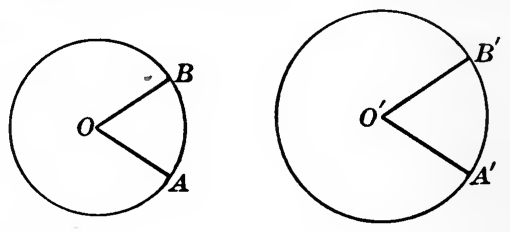
the centres $O$ and $O^{\prime}$ are equal.

368. Similar segments of circles are segments which have similar arcs.

Show that the angles inscribed in similar arcs or similar segments are equal. 
369. Similar sectors of circles are sectors which have similar ares, or which have equal angles between their radii.

370. Theorem. The area of a sector of a circle bears the same ratio to the area of the circle as the length of its arc bears to the whole circumference.

371. Conollary I. The area of a sector of a circle equals one-half the product of its arc and radius. .

Let the arc of a sector equal $a$ in a circle of radius $r$, and denote the area of the sector by $S$.

Then

$$
S: \pi r^{2}=a: 2 \pi r,
$$

that is

$$
S=\frac{1}{2} a r .
$$

372. Corollary II. The arcs of similar sectors are in the same ratio as the radii, and the areas of similar sectors are in the same ratio as the squares of the radii.

\section{EXERCISES}

1. The area of one circle is four times that of another. Show that the radius of the first is double that of the second.

2. The diameter of one circle is three times that of another; compare their circumferences and their areas.

3. A pond is fifty yards in diameter; what is the diameter of a pond having half the area?

4. The are of a sector of a circle equals the radius; show that the area of the sector is to the area of the circle in the ratio $1: 2 \pi$.

5. If the radius of a circle is 10 inches, find the length of a side of an inscribed equilateral triangle.

6. The area of the surface between two concentric circles is equal to twice the area of the smaller circle. Find the ratio between their radii.

7. Through any vertex of a regular pentagon two diagonals can be drawn. Find in degrees the angle between them. 


\section{SECTION III}

PROBLEMS

\section{Proposition X}

373. To inscribe a square in a given circle.

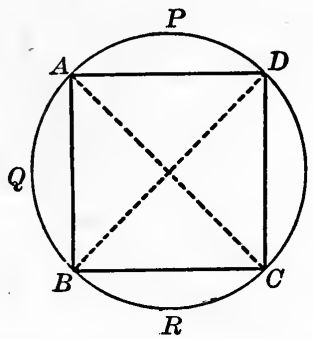

First, suppose $A B C D$ is the required square.

Draw the diagonals $A C$ and $B D$.

These are equal and bisect each other at right angles. Prove. Hence each is a diameter.

Therefore, to inscribe a square in a circle, draw two diameters at right angles and join their extremities in order.

Make the construction in this order and prove that the resulting figure is a square.

374. Theorem. If $r$ is the length of a radius of a given circle, and $a$ is the side of an inscribed square, then $a=r \sqrt{2}$.

The proof follows easily from the application of Proposition VII, Chapter IV.

375. Corollary. All squares inscribed in the same circle are identically equal.

\section{EXERCISES}

1. Inscribe a regular octagon (polygon of eight sides) in a given circle.

2. A square is inscribed in a circle of radius 3 inches ; find the length of each side and its distance from the centre of the circle. 


\section{Proposition XI}

- 376. To inscribe a regular hexagon in a given circle.

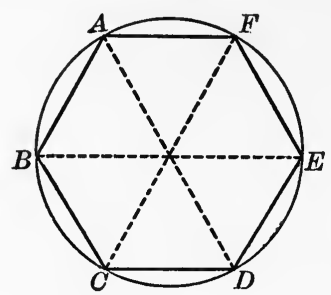

If $A B C D E F$ is the required hexagon, and $O$ the centre of the given circle, each side of the hexagon subtends at the centre an angle equal to $\frac{1}{6}$ of four right angles, or $\frac{2}{3}$ of one right angle. Therefore $\triangle A O B$ is equilateral, and $A B=O A$.

Hence, to construct a regular inscribed hexagon, mark off with the compasses ares of the circle whose chords are each equal to a radius, and join the points of division in order.

377. Theorem. The side of a regular inscribed hexagon is equal to a radius of the circle.

378. Theorem. If the alternate vertices of a regular inscribed hexagon be joined, the figure so formed is an inscribed equilateral triangle.

379. Theorem. The side of an inscribed equilateral triangle is equal to $r \sqrt{3}$, and its distance from the centre is $\frac{1}{2} r$.

380. Definition. The straight lines joining the pairs of opposite vertices in a polygon of an even number of sides are called the principal diagonals of the polygon.

\section{EXERCISES}

1. The pairs of opposite sides of a regular inscribed hexagon are parallel.

2. Each principal diagonal of a regular inscribed hexagon is a diameter. 


\section{Proposition XII}

381. To inscribe a regular decagon in a circle.

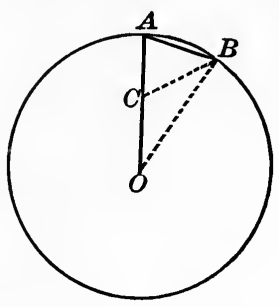

Construction. Take any radius $O A$, and divide it at $C$ in extreme and mean ratio, $O C$ being the greater segment, so that

$$
O A: O C=O C: C A \text {. }
$$

From $A$ draw a chord $A B$ equal to $O C$. Join $O B$ and $B C$.

Proof. Since $O A: A B=A B: A C, \triangle \mathrm{s} O A B$ and $B A C$ are similar.

(Art. 251.)

Therefore

$$
\angle A O B=\angle A B C .
$$

And $A B=B C$, since $\triangle B A C$ must be isosceles.

But

$$
\text { since } A B=O C, B C=O C \text {, }
$$

and

$$
\angle C O B \text { or } A O B=\angle C B O \text {. }
$$

That is $\angle \mathrm{s} A B C$ and $C B O$ are each equal to $\angle A O B$.

Therefore

$$
\begin{aligned}
& \angle A O B=\text { one-half } \angle O B A . \\
& \angle O A B=\angle O B A .
\end{aligned}
$$

Now

Therefore $\angle A O B$ is one-fifth of two right angles, or onetenth of four right angles.

Therefore $A B$ is one side of a regular inscribed decagon.

382. Theonem. If the alternate vertices of a regular inscribed decagon are joined, the figure so formed is a regular inscribed pentagon. 
383. Theorem. If from any point $A$ of a circle two chords $A B$ and $A C$ are drawn on the same side of $A$, the first a side of a regular inscribed decagon, and the second a side of a regular inscribed hexagon, then the chord $B C$ is a side of a regular inscribed polygon of fifteen sides.

Suggestion. What fraction of four right angles does the chord $A B$ subtend at the centre? $A C$ ? $B C$ ?

\section{Proposition XIII}

384. Given the radius of a circle and $a$ side of an inscribed regular polygon of $\boldsymbol{n}$ sides; to find the length of a side of an inscribed regular polygon of $\mathbf{2} \boldsymbol{n}$ sides.

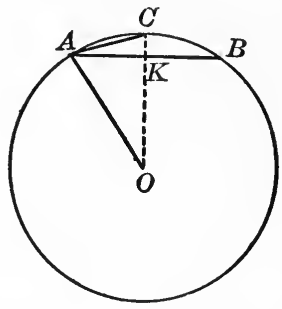

Let $O A(=r)$ be a radius of the given circle, $A B(=p)$ be a side of an inscribed regular polygon of $n$ sides, and $A C(=q)$ be a side of an inscribed regular polygon of $2 n$ sides. The problem is to find the value of $q$ in terms of $r$ and $p$.

Join $O C$. This line bisects $A B$ at right angles, at $K$.

Then

$$
\begin{aligned}
\overline{O K}^{2} & =\overline{O A}^{2}-\overline{A K}^{2} \\
& =r^{2}-\left(\frac{p}{2}\right)^{2} \\
& =\frac{4 r^{2}-p^{2}}{4},
\end{aligned}
$$

or

$$
O K=\frac{\sqrt{4 r^{2}-p^{2}}}{2},
$$

whence $K C=r-\frac{\sqrt{4 r^{2}-p^{2}}}{2}=\frac{2 r-\sqrt{4 r^{2}-p^{2}}}{2}$. 
Now

$$
\begin{aligned}
A C^{2} & =\overline{K C}^{2}+\overline{A K}^{2}, \\
q^{2} & =\left[\frac{2 r-\sqrt{4 r^{2}-p^{2}}}{2}\right]^{2}+\left(\frac{p}{2}\right)^{2} \\
& =2 r^{2}-r \sqrt{4 r^{2}-p^{2}} \\
q & =\sqrt{2 r^{2}-r \sqrt{4 r^{2}-p^{2}}} .
\end{aligned}
$$

whence

Writing $\frac{d}{2}$ instead of $r$, this formula becomes

$$
\begin{aligned}
q & =\sqrt{\frac{d^{2}}{2}-\frac{d}{2} \sqrt{d^{2}-p^{2}}} \\
& =\frac{1}{2} \sqrt{2 d^{2}-2 d \sqrt{d^{2}-p^{2}}} .
\end{aligned}
$$

If now we let $Q_{2 n}$ represent the perimeter of an inscribed regular polygon of $2 n$ sides, while $p$ represents the length of one side of a regular inscribed polygon of $n$ sides, we have

$$
Q_{2 n}=2 n q=n \sqrt{2 d^{2}-2 d \sqrt{d^{2}-p^{2}}}
$$

\section{EXERCISES}

1. Does the above formula for the value of $q$ hold true when $p=2 r$, in which case $q$ is a side of an inscribed square? Compare the result with that given on page 251 .

2. Apply the formula to find the side of an inscribed regular octagon.

3. In a circle of radius $r$, the side of an inscribed regular dodecagon equals $r \sqrt{2-\sqrt{3}}$.

4. In a circle of radius 3 feet, what is the length of a side of an inscribed regular dodecagon?

5. The area of a regular octagon inscribed in a circle is equal to the product of the sides of the inscribed and circumscribed squares.

6. The square of the side of an inscribed equilateral triangle is equal to the sum of the squares of the sides of the inscribed square and of the inscribed regular hexagon. 


\section{Proposition XIV}

385. To find approximately the value of $\pi$.

First, find the perimeter of a regular inscribed polygon of 12 sides.

In the formula of the preceding problem let

$$
2 n=12 \text {, then } n=6 \text {, and } p=r=\frac{d}{2} .
$$

Then

$$
\begin{aligned}
Q_{12} & =6 \sqrt{2 d^{2}-2 d \sqrt{d^{2}-\frac{d^{2}}{4}}} . \\
& =6 d \sqrt{2-\sqrt{3}} \\
& =d \times 3.105828 .
\end{aligned}
$$

Next, find the perimeter of a regular inscribed polygon of 24 sides.

$$
\begin{aligned}
& \text { If } 2 n=24, n=12 \text {, and } p=\frac{Q_{12}}{12}=d \times .258819 \text {, } \\
& \begin{aligned}
Q_{24} & =12 \sqrt{2 d^{2}-2 d \sqrt{d^{2}-(d \times .258819)^{2}}} \\
& =12 d \sqrt{2-2 \sqrt{1-(.258819)^{2}}} \\
& =d \times 3.132628 .
\end{aligned}
\end{aligned}
$$

Similarly,

$$
\begin{aligned}
& Q_{48}=d \times 3.139350 \\
& Q_{96}=d \times 3.141032 \\
& Q_{192}=d \times 3.141452 \\
& Q_{334}=d \times 3.141557 \\
& Q_{768}=d \times 3.141584 \\
& Q_{1536}=d \times 3.1415904
\end{aligned}
$$

When the number of sides of the inscribed polygon is indefinitely increased, $Q_{2 n}$ approaches the circumference $C$ as its limit.

But

$$
C=d \times \pi .
$$

(Art. 362.) 
Hence the multipliers of $d$ in the above computation are approximate values of $\pi$ corresponding to inscribed polygons of $12,24,48,96 \cdots$ sides, respectively.

The value $\pi=3.1415904$ is correct to five decimal places.

If the number of sides of the polygon were again doubled, a still closer approximation would be obtained.

The value of $\pi$ has been computed correctly to over seven hundred decimal places. In practice it is customary to use $\pi=3.1416$.

$\pi=\frac{22}{7}$ is an approximate value correct to two decimal places, while $\pi=\frac{355}{113}$ is correct to five decimal places.

A rough approximation to the value of $\pi$ can be obtained by rolling a circular piece of cardboard, of say 6 inches radius, along a straight line until it makes a complete revolution, then measuring carefully the length of the line-segment so traced, and comparing it with the diameter of the cardboard. A smaller error will probably be made if the cardboard is given two or three revolutions, and the whole distance divided by the number of revolutions.

A second method is to mark out a circle on a piece of cardboard ruled in squares, ordinary centimetre paper, then find approximately the area of the circle by counting the squares enclosed by it, reckoning each fractional square enclosed by the circle, a half square, this being an approximate average value. Setting this area equal to $\pi r^{2}$ gives an approximate value for $\pi$.

\section{EXERCISES}

In these exercises use $\frac{22}{7}$ as the value of $\pi$.

1. The diameter of a circle is 5 feet, what is its circumference?

2. The radius of a circle is 1 foot 8 inches, what is its circumference?

3. A wheel is twelve feet in circumference, what is its diameter?

4. A circular field is $\mathbf{1 0 0 0}$ yards in circumference, what is its diameter and its area?

5. Two fields are each 1600 yards around. One is circular and the other is square. What is the difference in their areas?

6. From a circular piece of paper of 10 inches radius, a circular piece is cut which has a radius of the first for its diameter. Find the area of the remaining piece. 


\section{MISCELLANEOUS EXERCISES}

1. Divide a given circle into two arcs such that any angle inscribed in one arc is three times an angle inscribed in the other.

Suggestion. The side of an inscribed square is the chord of the required arcs.

2. Divide a given circle into two arcs such that any angle inscribed in one arc is five times an angle inscribed in the other arc.

3. From a point without a circle two tangents are drawn which, with their chord of contact, form an equilateral triangle whose side is 18 inches. Find the diameter of the circle.

4. In a regular polygon of $n$ sides the straight lines which join any vertex to the non-adjacent vertices divide the angle at that vertex into $n-2$ equal parts.

5. An equilateral triangle and a regular hexagon are inscribed in a given circle; show that -

(1) The area of the triangle is half that of the hexagon;

(2) The square on a side of the triangle is three times the square on a side of the hexagon.

6. If $A B C D E$ is a regular pentagon and $A C, B E$ intersect at $H$, show that -

(1) $C H$ and $E H$ are each equal to a side of the pentagon ;

(2) $A B$ is a tangent to the circle circumscribed about the triangle $B H C$.

7. Show that the area of a regular hexagon inscribed in a circle is three-fourths of that of the corresponding circumscribed hexagon.

8. The area of a square circumscribed about a circle is double of the area of the inscribed square.

9. If $A B C D$ is a square inscribed in a circle and $P$ is any point on the circle, show that the sum of the squares on $P A, P B, P C, P D$ is double the square on the diameter.

10. An equilateral triangle is inscribed in a circle, and tangents are drawn at its vertices; prove that -

(1) The resulting figure is an equilateral triangle;

(2) Its area is four times that of the given triangle.

11. What is the area of a circular ring if the radii of the outer and inner circles are 132 and 120 feet, respectively?

12. A carriage wheel makes 168 revolutions in going half a mile. What is its height? 
13. The diameter of a circle is 12 feet. Find the diameter of a circle having (1) twice its circumference, (2) twice its area.

14. A circular pond is surrounded by a gravel walk, such that the area of the walk equals the area of the pond. What is the ratio of the diameter of the pond to the width of the walk?

15. A bicycle wheel is 28 inches in diameter. How many revolutions will it make in going 3 miles ?

16. Three circles are concentric and are such that the area of the first equals the area between the first and the second, and also between the second and third. The radius of the smallest is 10 inches. What are the radii of the other two ?

17. A circle is inscribed in a given square. What fraction of the area of the square lies outside of the circle? Is the fraction changed by enlarging the square and the inscribed circle?

18. A square is inscribed in a given circle. What fraction of the area of the circle lies outside of the square?

19. Show that the altitude of an equilateral triangle is to the radius of the circumscribed circle in the ratio of 3 to 2 .

20. Prove that the area of a circular ring is equal to the area of a circle whose diameter equals a chord of the outer boundary which is tangent to the inner.

21. Prove that any equilateral polygon circumscribing a circle is regular.

22. Each side of an inscribed equilateral triangle is parallel to the tangent at the opposite vertex.

23. Describe a circle whose area is equal to the sum of the areas of two given circles.

24. Describe a circle whose circumference is equal to the sum of the circumferences of two given circles.

25. Prove that the radius of an inscribed regular polygon is a mean proportional between its apothem and the radius of a similar circumscribed polygon.

26. If squares are described outwardly on the sides of a regular hexagon, prove that the outer vertices of the squares are the vertices of a regular dodecagon.

27. Find in degrees the angle at the centre of a circle of radius 2 feet, which is subtended by an arc whose length is 18 inches. 
1. Definitions.

\section{SUMMARY OF CHAPTER V}

(1) Regular Polygon - one which is both equilateral and equiangular. $\$ 335$.

(2) Centre of a Regular Polygon - the common centre of the inscribed and circumscribed circles. $\S 344$.

(3) Radius of a Regular Polygon - the radius of the circumscribed circle, i.e. the line from the centre to a vertex. $\$ 345$.

(4) Apothem of a Regular Polygon - the radius of the inscribed circle, i.e. the perpendicular from the centre on a side. $\$ 346$.

(5) Circumference of a Circle - the limit of the perimeter of a regular inscribed polygon as the number of its sides is indefinitely increased. $\$ 358$.

Or, the limit of the perimeter of a regular circumscribed polygon as the number of its sides is indefinitely increased. $\$ 360$.

(6) Length of an Arc of a Circle - the limit of the sum of chords in the arc as the number of such chords is indefinitely increased. $\S 358$.

(7) Area of a Circle - the surface enclosed by the circle, equal to the limit of the area of a regular inscribed polygon as the number of its sides is indefinitely increased. $\$ 359$.

(8) Similar Arcs of Circles - arcs which subtend equal angles at the centres of the circles. $\$ 367$.

(9) Similar Segments - those which have similar arcs. $\$ 368$.

(10) Similar Sectors - those which have similar arcs. $\$ 369$.

(11) Principal Diagonals of a Polygon - the diagonals joining pairs of opposite vertices in a polygon of an even number of sides. $\S 380$.

(12) Pentagon, Hexagon, Octagon, Decagon, Dodecagon. See $§ 336$.

2. Postulates.

(1) A circle may be divided into any given number of equal parts. (Postulate 8.) §337.

3. Problems.

(1) To inscribe a square in a given circle. $\$ 373$.

(2) To inscribe a regular hexagon in a given circle. $\$ 376$.

(3) To inscribe an equilateral triangle in a given circle. $\$ 378$.

(4) To inscribe a regular decagon in a given circle. $\$ 381$.

(5) To inscribe a regular pentagon in a given circle. $\$ 382$. 
(6) To inscribe a regular polygon of fifteen sides in a given circle. $\S 383$.

(7) To find the length of a side of an inscribed regular polygon of $2 n$ sides, having given the length of the side of the inscribed polygon of $n$ sides. $\S 384$.

(8) To find approximately the ratio of the circumference to the diameter of a circle, i.e. the value of $\pi$. $\$ 385$.

The last two are numerical rather than geometrical problems.

\section{Theorems on Inscribed and Circumscribed Regular Polygons.}

(1) If a circle is divided into any number of equal arcs, (1) the chords joining the points of division taken in order form a regular inscribed polygon, (2) the tangents to the circle at the points of division taken in order form a regular circumscribed polygon. $\$ 339$.

(2) An equilateral polygon inscribed in a circle is also equiangular, and hence regular. $\S 342$.

(3) A circle can be circumscribed about any regular polygon, and another circle can be inscribed in it. $\$ 343$.

(4) If the number of sides of a regular inscribed polygon be doubled, the perimeter will be increased, but if the number of sides of a regular circumscribed polygon be doubled, the perimeter will be diminished. $\$ 354$.

(5) The area of a regular inscribed polygon is increased, and the area of a regular circumscribed polygon is diminished, when the number of sides is doubled. $\S 355$.

(6) The side of an inscribed square is equal to $r \sqrt{2}$. $\$ 374$.

(7) All squares inscribed in the same circle are identically equal. $\S 375$.

(8) The side of a regular inscribed hexagon is equal to a radius of the circle. $\$ 377$.

(9) The side of an inscribed equilateral triangle is equal to $r \sqrt{3}$, and its distance from the centre is $\frac{1}{2} r . \quad \S 379$.

5. Theorems on the Properties of Regular Polygons.

(1) Any radius of a regular polygon bisects the angle at the vertex. $\S 347$.

(2) The angle formed by two consecutive radii of a regular polygon equals four right angles divided by the number of sides of the polygon. $\$ 348$.

(3) Any two regular polygons of the same number of sides are similar. $\$ 349$. 
(4) Homologous sides in two regular polygons of the same number of sides are in the same ratio as the radii of the circumscribed circles, or as the radii of the inscribed circles; that is, as the radii of the polygons or as the apothems of the polygons. $\S 350$.

(5) The perimeters of two regular polygons of the same number of sides are in the same ratio as their radii, or as their apothems. $\S 351$.

(6) The areas of two regular polygons of the same number of sides are in the same ratio as the squares of their radii, or as the squares of their apothems. $\S 352$.

(7) The area of a regular polygon is equal to half the product of its perimeter and its apothem. $\$ 353$.

6. Theorems on the Circumferences and Areas of Circles.

(1) The circumference of a circle is greater than the perimeter of any regular inscribed polygon, and less than the perimeter of any regular circumscribed polygon. $\S 361$.

(2) The ratio of the circumference of a circle to its diameter is the same for all circles. $C=\pi d=2 \pi r . \quad \S 362$.

(3) The area of a circle is equal to one-half the product of its circumference and radius. $\$ 364 . \quad A=\frac{1}{2} C r=\pi r^{2}=\frac{1}{4} \pi d^{2} . \quad \S 365$.

(4) The areas of two circles are in the same ratio as the squares of their radii, or as the squares of their diameters. $§ 366$.

\section{Theorems on Sectors of a Circle.}

(1) The area of a sector of a circle bears the same ratio to the area of the circle as the length of its arc bears to the whole circumference. $§ 370$.

(2) The area of a sector of a circle equals one-half the product of its arc and radius. $S=\frac{1}{2}$ ar. $\$ 371$.

(3) The arcs of similar sectors are in the same ratio as the radii, and the areas of similar sectors are in the same ratio as the squares of the radii. $\S 372$. 


\section{PART II - SOLID GEOMETRY}

CHAPTER VI

LINES AND PLANES IN SPACE

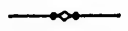

\section{SeCtion I}

INTERSECTING PLANES-PARALLELS AND PERPENDICULARS

386. We come now to the study of geometrical figures whose points and lines do not all lie in the same plane. Such a figure is called a solid figure, or a figure of three dimensions.

A solid figure is spread out or extended in three ways.

A plane figure is two-dimensional since it is spread out in but two ways.

A straight line is one-dimensional since it has only length.

387. It should be remembered that in geometry we are concerned only with forms and relations, not at all with matter; and so it is necessary to distinguish between a three-dimensional figure, or a so-called solid figure, and a physically solid body.

A solid figure is a combination of points, lines, and surfaces. It contains no matter.

Since in Solid Geometry the diagrams are intended to represent figures which do not lie wholly in one plane, they are drawn as we say in perspective, to give an idea of how the figure would look at a distance. Straight lines and planes are supposed to extend indefinitely in any of their directions, though they must be represented in a diagram by limited portions of a line or a plane. For the most part lines are dotted when they are supposed to be seen through a surface which forms a part of the figure. 
388. When we said (p. 3) that two points determine a straight line, we meant that through two points there can pass one straight line, and only one.

In just the same way, three points which do not lie on the same straight line determine a plane, and the possibility of constructing this plane is assumed.

Postulate 9. Through three points not in the same straight line there can be passed one and only one plane.

If then we name three points in any plane, not lying in the same straight line, they will be sufficient to distinguish this plane from every other.

Through two points (or, through the straight line joining two points), any number of planes can be passed.

It follows immediately that

$A$ straight line and a point not lying on it determine a plane.

If two points are chosen on the given line, these two and the given point determine the plane. Does the given line lie wholly in that plane? (See definition of a plane, p. 4.)

Two intersecting straight lines determine a plane.

The point of intersection and one other point on each line determine the plane. Do each of the given lines lie in that plane?

Two parallel straight lines determine a plane.

For, two parallel straight lines lie in one plane by definition (Art. 85), and this must be the plane determined by one of them and a point of the other.

389. Two straight lines which intersect have in common a single point. A straight line and a curved line, or two curved lines, may have in common more than one point.

A straight line and a surface intersect in one or more points. If the surface is plane a straight line can intersect it in but one point.

Two surfaces intersect in one or more lines. These contain all points common to the two surfaces. 


\section{Proposition I}

390. The intersection of two planes is a straight line.

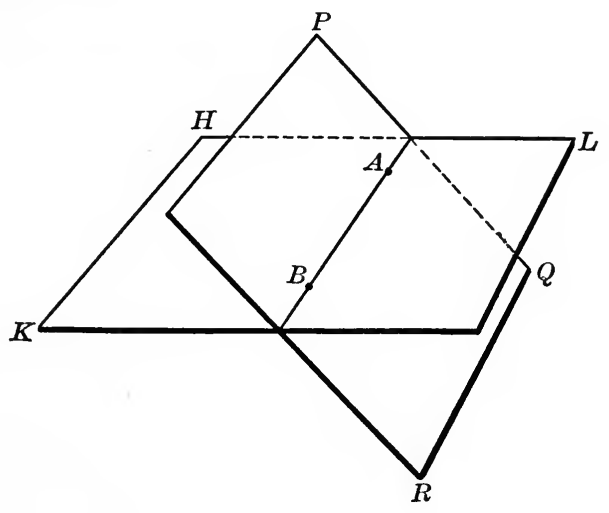

Let $H K L$ and $P Q R$ be any two intersecting planes.

It is required to prove that their intersection is a straight line, or in other words, that the locus of points common to the two planes is a straight line.

Proof. First, let $A$ and $B$ be any two points common to the planes $H K L$ and $P Q R$.

From the definition of a plane every point of the straight line $A B$ lies in the plane $H K L$.

For the same reason, every point of the straight line $A B$ lies in the plane $P Q R$.

Therefore the straight line $A B$ is common to the two planes.

Next, no point outside of $A B$ can be common to the two planes, for then the two planes would coincide.

(Art. 388.)

Therefore the intersection of the planes $H K L$ and $P Q R$ is a straight line.

When no confusion is likely to arise a plane may be denoted by a single letter, as $H$, or $P$, in the above diagram. 
391. Theorem. If two planes have one point in common they must have a second point, and hence a straight line, in common.

Let the planes $M$ and $N$ have the point $A$ in common.

To prove that they must have a second point in common.

In the plane $N$ choose two points $B$ and $C$ on the same side of the plane $M$. Join $B A$ and produce to $F$, so that $B$ and $F$ are on opposite sides

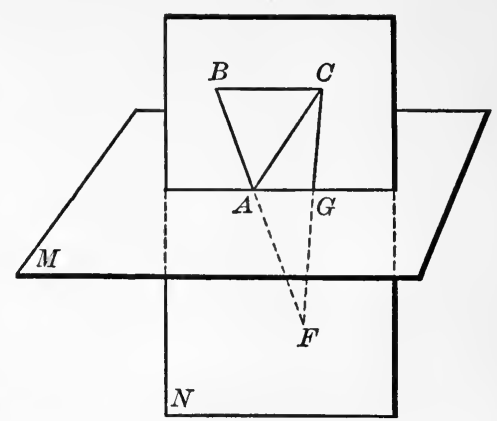
of $M$. Then $C$ and $F$ are also on opposite sides of $M$, and the straight line $C F$ must penetrate the plane $M$ at some point $G$ different from $A$. But since $C F$ lies wholly in the plane $N$, the point $G$ is common to the two planes.

By the preceding theorem the straight line $A G$ is common to the two planes and is the locus of their common points.

\section{Definitions}

392. If a straight line and a given plane do not meet, however far they are extended, they are said to be parallel.

A straight line not lying in a given plane, and not parallel to it, intersects the plane at one point. For convenience, this point is sometimes called the foot of the line.

393. A straight line is perpendicular to a given plane when, and only when, it is perpendicular to every straight line in the plane, drawn through their point of intersection. In that case the plane is also said to be perpendicular to the line.

A straight line neither parallel nor perpendicular to a given plane is said to be oblique to it.

Two planes are parallel when they do not ineet, however far they may be extended. 


\section{Proposition II}

394. If three planes intersect, two and two, their three lines of intersection are either concurrent, or are parallel two and two.

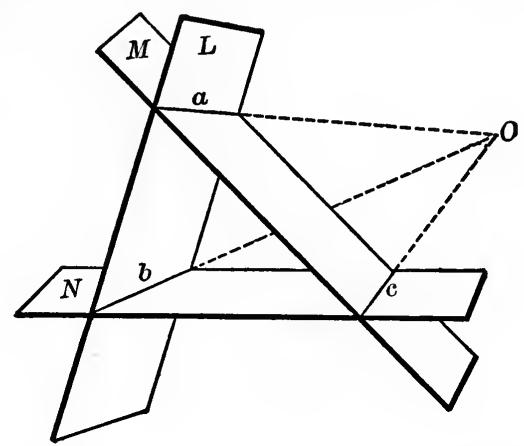

Let $L, M$, and $N$ be the three given planes, and let $a$ be the intersection of $L$ and $M, b$ the intersection of $L$ and $N$, and $c$ the intersection of $M$ and $N$.

It is required to prove that the straight lines $a, b$, and $c$ all pass through one point, or are parallel, two and two.

Proof. First, suppose the lines $a$ and $b$, which both lie in the plane $L$, to intersect at a point $O$. Then because $O$ is a point of $a$ it lies on the plane $M$, and because it is a point of $b$ it lies on the plane $N$.

Therefore, $O$ is a point of $c$, the intersection of $M$ and $N$.

That is, $a, b$, and $c$ all pass through one point.

Next, suppose $a$ and $b$ are parallel.

Then, if $a$ and $c$ are not parallel, they must intersect, since they both lie in the plane $M$; and it may be shown just as before that their common point lies upon $b$, which is impossible since $a$ and $b$ are parallel.

Therefore

Similarly, $a$ and $c$ must be parallel.

$b$ and $c$ must be parallel. 


\section{Proposition III}

395. The two planes determined by two given parallel lines and a point not lying in their plane intersect in a line parallel to each of the given lines.

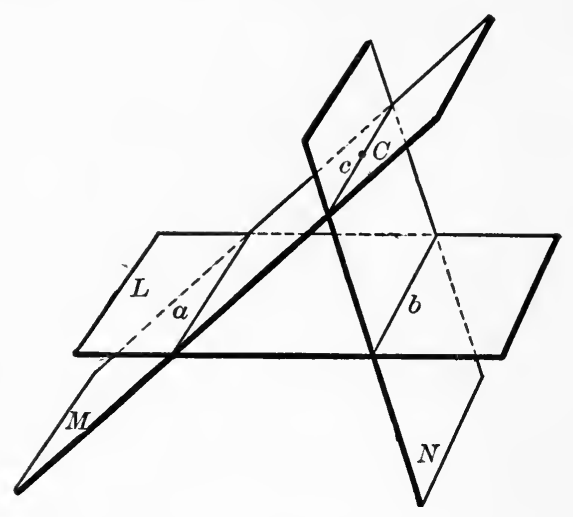

Let $a$ and $b$ be the given parallel lines lying in the plane $L$, and let $C$ be the given point.

Also, let $M$ be the plane determined by $C$ and $a, N$ the plane determined by $C$ and $b$, and let $M$ and $N$ intersect in the line $c$.

It is required to prove that $c$ is parallel to both $a$ and $b$.

Proof. The planes $L, M$, and $N$ intersect, two and two, in the straight lines $a, b$, and $c$.

These lines must therefore be concurrent, or be parallel two and two.

(Prop. II.)

They cannot be concurrent, since $a$ and $b$ are parallel, by hypothesis.

Therefore $c$ must be parallel to both $a$ and $b$.

396. Corollary. Two straight lines each parallel to a third line, are parallel to each other. 


\section{Proposition IV}

397. If a straight line is perpendicular to each of two given straight lines at their point of intersection, it is perpendicular to the plane of those lines.

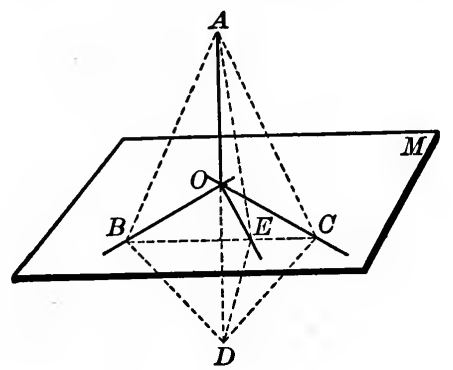

Let the straight line $A O$ be perpendicular to both the straight lines $B O$ and $C O$ at their common point $O$, and let $M$ be the plane determined by $B O$ and $C O$.

It is required to prove that $A O$ is perpendicular to the plane $M$.

Proof. Suppose $O E$ is any other straight line drawn in the plane $M$, through the point $O$. Then if $A O$ is perpendicular to $O E$, it is perpendicular to the plane $M$.

(Art. 393.)

Draw a straight line in the plane $M$ cutting $B O$ and $C O$ at $B$ and $C$, and the line $O E$ at $E$.

Join $A B, A E, A C$.

Produce $A O$ to $D$, making $O D$ equal to $O A$.

Join $D B, D E, D C$.

Since $O B$ is a perpendicular bisector of the line-segment $A D$, $B A$ equals $B D$.

(Art. 72.)

Similarly $C A$ equals $C D$, and hence, $\triangle \mathrm{s} A B C$ and $D B C$ are identically equal.

(Art. 53.)

If these two triangles were superposed, $D E$ would coincide with $A E$, and is therefore equal to $A E$. 
Since $E$ and $O$ are each equidistant from $A$ and $D, E O$ must be a perpendicular bisector of $A D$.

Therefore $\angle A O E$ is a right angle, and consequently $A O$ is perpendicular to the plane $\boldsymbol{M}$.

398. Corollary I. At any point of a straight line one plane can be constructed perpendicular to that line, and only one.

First, two perpendiculars, $O B$ and $O C$, in different planes, can be drawn to the given line $A D$ at the given point $O$, and the plane of these is perpendicular to the given line (Prop. IV).

Next, if any plane other than the plane $B O C$ can be constructed perpendicular to $A D$ at $O$, say the plane $B O C^{\prime}$, it must cut the line $A C$ in a point $C^{\prime}$ different from $C$, and the line $O C^{\prime}$ must then be perpendicular to $A O$, which is impossible, since $O C$ lies in the same plane as $O C^{\prime}$ and is perpendicular to $A D$ at $O$.

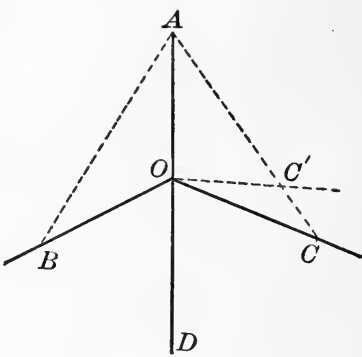

399. Corollary II. Through a given point not on a given straight line, one plane and only one can be constructed perpendicular to the given line.

Let $P$ be the given point and $a$ the given straight line. In the plane $P a$ draw $P A$ perpendicular to $a$, and from $A$ draw another line $A Q$ perpendicular to $a$. The plane of $A P$ and $A Q$ passes through $P$ and is perpendicular to $a$ (Prop. IV). No other plane can pass through $P$ and be perpendicular to $a$, for such a plane must either intersect $a$ at $A$ and not contain $A Q$, or else it must intersect $a$ at a point different from $A$, both of which suppositions are impossible. Show why?

400. Corollary III. Two intersecting planes cannot both be perpendicular to the same straight line.

401. Corollary IV. All the straight lines perpendicular to a given line at a given point lie in one plane perpendicular to the given line. 
Let $O B$ and $O C$ be two lines perpendicular to the straiglit line $A D$ at $O$. Their plane is perpendicular to $A D$ at $O$. Let $O E$ be any other straight line perpendicular to $A D$ at $O$, and suppose, if possible, that it does not lie in the plane $B O C$. 'Then the plane $B O C$ will cut the plane $A O E$ in a straight line $O E^{\prime}$ different from $O E$. Since $O E^{\prime}$ lies in the plane $B O C, \angle A O E^{\prime}$ is a right angle. But this is impossible since $\angle A O E$ is a right angle. Therefore $O E$ cannot lie out of the plane $B O C$.

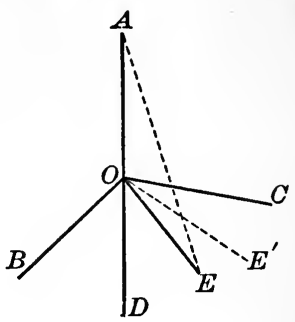

402. Corollary V. If a plane bisects a given line-segment perpendicularly, every point of the plane is equidistant from the extremities of the line-segment.

And conversely: Every point which is equidistant from two fixed points lies in the plane bisecting perpendicularly the line joining them.

\section{EXERCISES}

1. Through four points not lying in the same plane, how many planes can be passed, each containing three of the points? In how many of these planes would any one of the given points lie?

2. Through five points, no four of which lie in the same plane, how many planes can be passed, each containing three of the points?

3. Show how to draw through a given point a straight line which will intersect two given lines not lying in the same plane. Can more than one such line be drawn?

Definition. Two straight lines so situated that no plane can contain them both are said to be skew or gauche to each other.

Hold two pencils so that they are gauche to each other.

4. Find the locus of points in a plane which are equidistant from two given points not lying in the plane.

5. Find a point in a plane equidistant from three given points not lying in that plane.

6. A three-legged table will always stand firmly on a level floor, while a four-legged table will not. How do you account for this? 


\section{Proposition V}

403. At a given point in a given plane to erect a perpendicular to the plane.

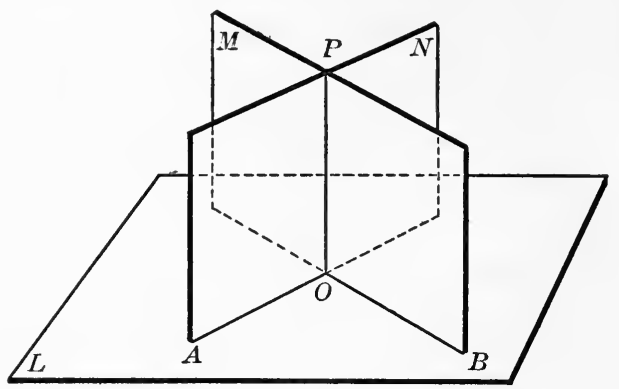

Let $L$ be the given plane and $O$ the given point in it.

It is required to draw through $O$ a straight line perpendicular to the plane $L$.

Construction. Through $O$ draw any two straight lines $O A$ and $O B$ in the plane $L$. At $O$ construct a plane $M$ perpendicular to $O A$, and a plane $N$ perpendicular to $O B$.

(Art. 398.)

These two planes, $M$ and $N$, will intersect in a straight line $O P$ which is perpendicular to $L$.

Proof. Since $O P$ lies in the plane $M$ and passes through $O$, it is perpendicular to $O A$.

(Art. 393.)

And since it lies in the plane $N$, it is perpendicular to $O B$. Therefore $O P$ is perpendicular to the plane $L$. (Prop. IV.)

404. Theorem. At a point in a plane, but one straight line can be drawn perpendicular to the plane.

If there were a second straight line $O Q$ perpendicular to the plane $L$, the plane of $O P$ and $O Q$ would intersect $L$ in a straight line $O R$, to which $O P$ and $O Q$ are both perpendicular. But this is impossible.

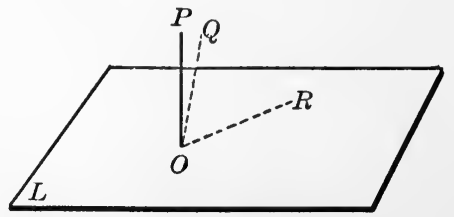




\section{Proposition VI}

405. Two straight lines perpendicular to the same plane are parallel.

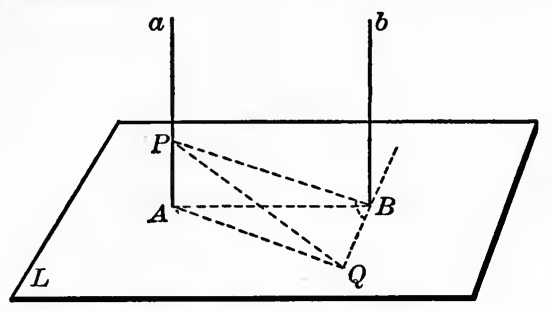

Let $a$ and $b$ be straight lines perpendicular to the plane $L$, at the points $A$ and $B$, respectively.

It is required to prove that $a$ and $b$ are parallel.

Proof. First, to show that $a$ and $b$ are in the same plane. Join $A B$, and in the plane $L$ draw $B Q$ perpendicular to $A B$. In $a$ choose any point $P$, and make $B Q$ equal to $A P$. Join $P B, P Q$, and $A Q$.

$\triangle P A B$ is identically equal to $\triangle Q B A$. Why? Therefore $B P=A Q$.

Also, $\triangle P B Q$ is identically equal to $\triangle Q A P$. Why? Therefore $\angle P B Q$ is a right angle.

Hence $b, B P$, and $B A$ must lie in one plane. (Art. 401.) But since $a$ meets both $B P$ and $B A$, it also must lie in that plane.

Next, the interior angles which $a$ and $b$ make with the transversal $A B$ are both right angles.

Therefore $a$ and $b$ are parallel.

(Art. 90.)

406. CoRollary I. If from the foot of a given perpendicular to a plane, a straight line is drawn at right angles to any line in the plane, any line through their intersection, which meets the given perpendicular, is at right angles to the line of the plane.

That is, $B P$ drawn to meet $a$ is perpendicular to $B Q$. 
407. Corollary II. If one of two parallel lines is perpendicular to a plane, the other is also.

For, if not, at its point of intersection with the plane erect a perpendicular. This lies in a plane with the first perpendicular and is parallel to it.

\section{Proposition VII}

408. From a given point without a given plane to draw a perpendicular to the plane.

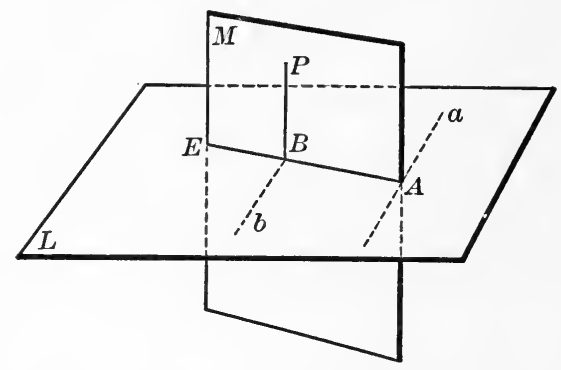

Let $L$ be the given plane, and $P$ the given point without it.

It is required to draw from $P$ a straight line perpendicular to the plane $L$.

Construction. In $L$ take any straight line $a$, and through $P$ construct the plane $M$ perpendicular to $a$.

(Art. 399.)

This plane will intersect $L$ along some line $A E$.

From $P$ in the plane $M$ draw $P B$ perpendicular to $A E$.

$P B$ is the required line.

Proof. From $B$ in the plane $L$ draw the straight line $b$ parallel to $a$.

Then $b$ is perpendicular to the plane $M$ (Art. 407), and hence perpendicular to $P B$ which lies in $\boldsymbol{M}$.

But $P B$ was drawn perpendicular to $A E$.

Therefore $P B$, being perpendicular to both $A E$ and $b$, is perpendicular to their plane, namely, the plane $L$. 
409. Theorem. From a point without a plane only one perpendicular to the plane can be drawn.

For if there are two perpendiculars from $P$, their plane will intersect the given plane in a straight line perpendicular to them both, which is impossible.

410. Corollary. Of all straight

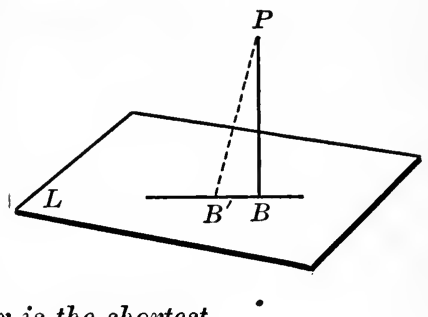
lines which can be drawn from a point to a plane, the perpendicular is the shortest.

411. Definition. The distance from a point to a plane is the length of the perpendicular from the point to the plane.

\section{EXERCISES}

1. $A B$ and $C D$ are two straight lines of which $A B$ lies in a plane perpendicular to $C D$. Prove that the perpendiculars to $A B$ from the different points of $C D$ all pass through one point.

Suggestion. Compare this exercise with the theorem stated in Article 406. What is the relation between them?

2. If $D$ is any point on the straight line drawn through the orthocentre of a triangle $A B C$, perpendicular to the plane of the triangle, then $D A$ is at right angles to the straight line drawn through $A$ parallel to $B C$.

3. Find the locus of points in space equidistant from the points of a circle.

4. Equal oblique lines drawn from a point to a plane make equal angles with the perpendicular from the point, and meet the plane at equal distances from the foot of the perpendicular. Of two unequal lines so drawn, which makes the greater angle with the perpendicular?

5. If two straight lines are each parallel to a third line, they are parallel to each other. Prove. (See Article 396.)

Suggestion. A plane perpendicular to the third line is perpendicular to each of the others.

6. If two straight lines are not in the same plane, no two straight lines joining points of the one to points of the other can be parallel. 


\section{Proposition VIII}

412. Two planes perpendicular to the same straight line are parallel.

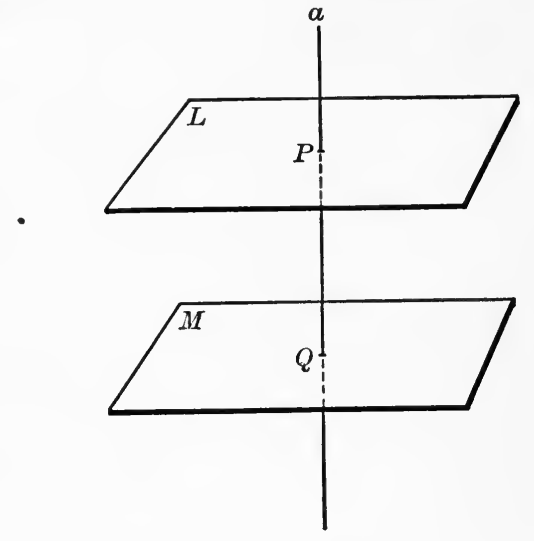

Let $L$ and $M$ be two planes, each perpendicular to the straight line $a$, at the points $P$ and $Q$, respectively.

It is required to prove that these planes are parallel.

Proof. If $L$ and $M$ are not parallel they must intersect in some straight line. Let $E$ be any point of their intersection.

Then through $E$ there would pass two planes perpendicular to $a$, which is impossible.

(Art. 399.)

Therefore $L$ and $M$ are parallel.

\section{EXERCISES}

1. If two planes are perpendicular to the same straight line, any plane containing that line intersects them in parallel lines.

2. Find the locus of points equidistant from two given parallel planes.

3. Prove that through a given point outside a given plane, any required number of straight lines can be drawn parallel to the given plane. 


\section{Proposition IX}

413. If two straight lines are parallel, any plane containing one of them, and not the other, is parallel to the other.

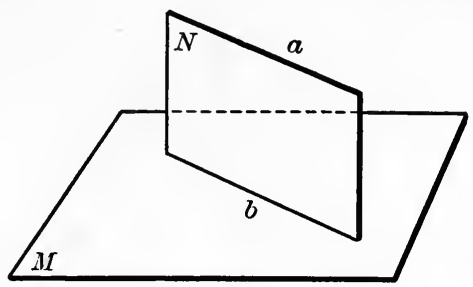

Let $a$ and $b$ be two parallel straight lines, and let the plane $M$ contain the line $b$, but not $a$.

It is required to prove that the plane $M$ is parallel to the line $\alpha$.

Proof. Since $a$ and $b$ are parallel, they lie in one plane by definition, say the plane $N$.

All the points common to $M$ and $N$ lie on the straight line $b$.

(Prop. I.)

Therefore, if $a$ intersects $M$ at any point, it must be at some point of $b$.

But $a$ is parallel to $b$, by hypothesis, and therefore, can have no point in common with $b$, or with the plane $\boldsymbol{M}$. Therefore $\boldsymbol{M}$ is parallel to $a$.

414. Corollary I. Through either of two given straight lines not lying in the same plane, one plane can be passed parallel to the other line.

If $a$ and $b$ are the given straight lines, from any point $P$ of $b$ draw $P Q$ parallel to $a$. Then the plane of $b$ and $P Q$ is parallel to $a$, and passes through $b$.

Can more than one plane be so passed through $b$ ?

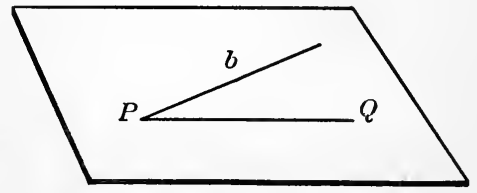


415. Corolitary II. Through a given point, a plane can be passed parallel to any two given straight lines in space.

Suggestion. Through the given point draw straight lines parallel to the given straight lines.

\section{Proposition $\mathrm{X}$}

416. If a straight line is parallel to a given plane, it is parallel to the intersection of any plane through it, with the given plane.

The proof is left to the student with the following queries:

(1) Are the two straight lines under consideration in the same plane?

(2) Will they meet if produced?

417. Conollary. If a straight line is parallel to a given plane, a line drawn from any point in the plane parallel to the given line lies in the given plane.

Suggestion. The chosen point and the given line determine a plane.

\section{EXERCISES}

1. If from any point perpendiculars are drawn to two parallel planes, these perpendiculars must coincide.

2. Prove that if one of two parallel planes is perpendicular to a given straight line, the other must be.

3. Prove that if through each of two parallel straight lines, a plane is passed, these two planes will intersect, if at all, in a straight line parallel to both the given lines.

4. If $a$ and $b$ are two straight lines which do not lie in the same plane, and $c$ and $d$, two straight lines meeting them, prove that either $c$ and $d$ intersect on $a$, or on $b$, or else have no common plane.

5. If two straight lines are parallel, any straight line joining a point in one of them to a point in the other lies in the plane determined by the two parallel lines. 


\section{Proposition XI}

418. If two intersecting straight lines are each parallel to a given plane, the plane determined by these lines is also parallel to the given plane.

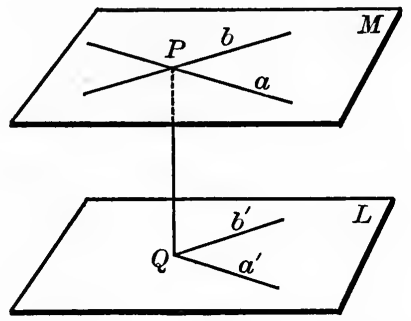

Let $a$ and $b$ be two straight lines, intersecting at $P$, which are each parallel to the plane $L$.

It is required to prove that the plane $M$, determined by $a$ and $b$, is also parallel to $L$.

Proof. From $P$ draw a perpendicular to the plane $L$, meeting it at $Q$.

(Prop. VII.)

From $Q$ draw $a^{\prime}$ and $b^{\prime}$ parallel to $a$ and $b$, respectively.

These will lie in the plane $L$ (Prop. X, Cor.) and hence be perpendicular to $P Q$.

The straight lines $a$ and $b$, being parallel to $a^{\prime}$ and $b^{\prime}$ respectively, are also perpendicular to $P Q$.

Hence the plane $M$ is perpendicular to $P Q$.

But $P Q$ was drawn perpendicular to $L$.

(Art. 97.)

Therefore the planes $L$ and $M$ are parallel to each other.

(Prop. VIII.)

\section{EXERCISES}

1. If three straight lines intersect at one point, and all meet a fourth straight line not through that point, the first three must lie in one plane.

2. If three straight lines are such that each intersects the other two, they must all lie in one plane, or must all pass through one point. 


\section{Proposition XII}

419. Two parallel planes are intersected by any third plane in parallel lines.

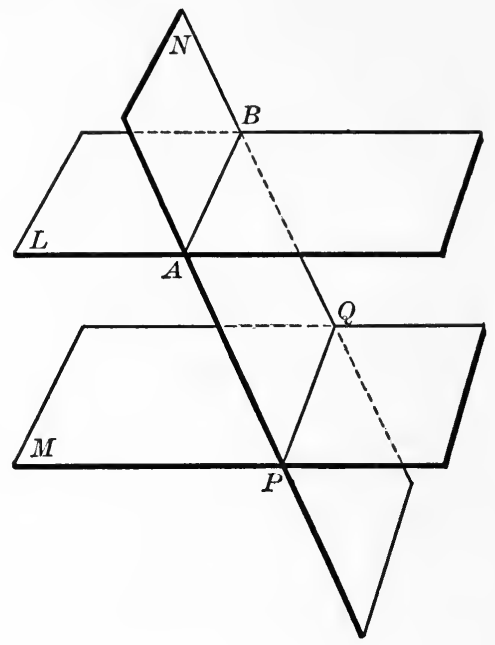

Let $L$ and $M$ be any two parallel planes, and let $N$ be a third plane intersecting $L$ in the straight line $A B$, and $M$ in the straight line $P Q$.

It is required to prove that $A B$ and $P Q$ are parallel.

Proof. First, $A B$ and $P Q$ lie in the same plane $N$.

Next, $A B$ and $P Q$ cannot meet since the planes $L$ and $M$ have no point in common however far they are extended.

Therefore $A B$ and $P Q$ are parallel.

420. Corollary I. Parallel line-segments terminated by parallel planes are equal.

Pass a plane through the two parallel line-segments. The two given line-segments and the intersections of this plane with the parallel planes form a parallelogram. 
421. Corollary II. Two parallel planes are everywhere equidistant.

422. Corollary III. A straight line perpendicular to one of two parallel planes is also perpendicular to the other.

Pass two planes through the given line and study their intersections with the given planes.

423. Corollary IV. Through any point one plane can be passed parallel to a given plane, and only one.

Let $P$ be the given point and $L$ the given plane.

From $P$ draw $P Q$ perpendicular to $L$.

At $P$ construct a plane $M$ perpendicular to $P Q$.

Then $M$ is parallel to $L$. (Prop. VIII.)

If there is a second plane through $P$ parallel to $L, P Q$ must also be perpendicular to it (Cor. III). This is impossible.

(Art. 400.)

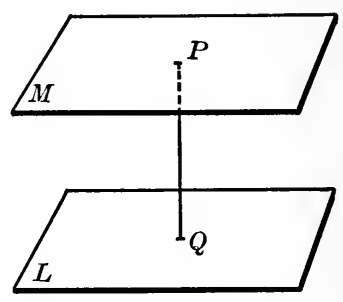

\section{EXERCISES}

1. Three planes in general intersect at one point. Can three planes have a straight line in common without coinciding?

2. There is one rectilinear figure which is necessarily plane. What is it?

3. Four planes are such that no three of them pass through the same straight line and no four of them contain the same point. In how many straight lines do they intersect, two and two? In how many points do they intersect, three and three? How many lines of intersection through each point? How many points of intersection on each line?

4. $A$ and $B$ are two points on the same side of a plane, $P$ a point in the plane. Determine $P$ so that the sum of the line-segments $A P$ and $P B$ shall be the least possible.

5. If four planes are given, and the common line of the first two intersects the common line of the last two, the four planes meet in one point. 


\section{Proposition XIII}

424. If two intersecting straight lines lying in one plane are parallel, respectively, to two intersecting straight lines lying in another plane, the two planes must be parallel, and the angles formed by the lines are equal.

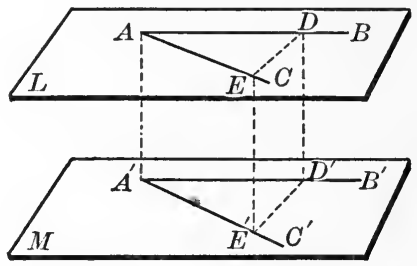

Let the straight lines $A B$ and $A C$, lying in the plane $L$, be parallel, respectively, to the straight lines $A^{\prime} B^{\prime}$ and $A^{\prime} C^{\prime}$, lying in the plane $M$, and let them extend in the same way from the vertex.

It is required to prove that the planes $L$ and $\boldsymbol{M}$ are parallel, and that the angle $B A C$ equals the angle $B^{\prime} A^{\prime} C^{\prime}$.

Proof. First, the lines $A B$ and $A C$ are both parallel to the plane $\boldsymbol{M}$.

(Prop. IX.)

Therefore the plane $L$ is parallel to the plane $M$. (Prop. XI.)

Next, from $A B$ and $A^{\prime} B^{\prime}$ cut off equal segments $A D$ and $A^{\prime} D^{\prime}$.

Also from $A C$ and $A^{\prime} C^{\prime \prime}$ cut off equal segments $A E$ and $A^{\prime} E^{\prime}$. Join $A A^{\prime}, D D^{\prime}, E E^{\prime}$, also $D E$ and $D^{\prime} E^{\prime}$.

$A A^{\prime} D^{\prime} D$ is a parallelogram. Why?

Therefore $D D^{\prime}$ is equal and parallel to $A A^{\prime}$.

$A A^{\prime} E^{\prime} E$ is also a parallelogram. Why?

Therefore $E E^{\prime}$ is equal and parallel to $A A^{\prime}$.

Hence $E E^{\prime}$ is equal and parallel to $D D^{\prime}$.

(Art. 396.)

Therefore $E E^{\prime} D^{\prime} D$ is a parallelogram, and $E D$ is equal to $E^{\prime} D^{\prime}$.

Therefore $\triangle D A E$ is identically equal to $\triangle D^{\prime} A^{\prime} E^{\prime}$ (Art. 53), and $\angle D A E$ is equal to $\angle D^{\prime} A^{\prime} E^{\prime}$. 


\section{Proposition XIV}

425. If two straight lines are cut by three parallel planes, the corresponding segments are proportional.

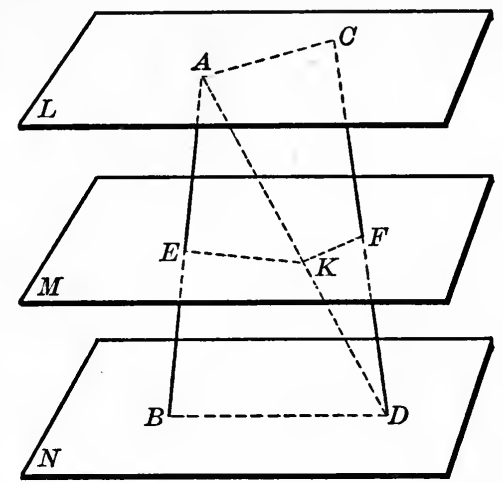

Let $A B$ and $C D$ be two straight lines which are intersected by the parallel planes $L, M, N$, in the points $A, E, B$ and $C, F, D$, respectively.

It is required to prove that $A E: E B=C F: F D$.

Proof. Draw the straight line $A D$ cutting the plane $M$ at the point $K$.

Join $E K$ and $K F$, also $A C$ and $B D$.

Now, the plane $B A D$ must intersect $M$ and $N$ in parallel lines.

(Prop. XII.)

Therefore $E K$ is parallel to $B D$.

Hence

$$
A E: E B=A K: K D \text {. }
$$

Similarly, $A K: K D=C F: F D$.

Therefore

$$
A E: E B=C F: F D \text {. }
$$

\section{EXERCISES}

1. If $n$ parallel planes intercept equal segments on one straight line, they will intercept equal segments on any other straight line. 


\section{SECTION II}

\section{DIHEDRAL ANGLES}

426. When two planes intersect they are said to form a dihedral angle.

The line of intersection of the planes is called the edge of the dihedral angle, and the planes are the boundaries or faces of the angle.

By rotating one of the planes about their line of intersection, the other being held stationary, the dihedral angle is increased or diminished, according as the plane is rotated in the one way, or in the other.

A dihedral angle may be designated by mentioning two points in its edge and one other point in

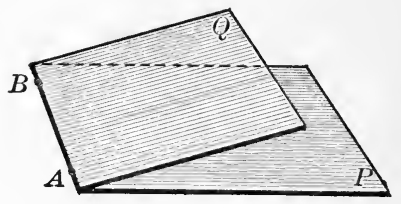
each boundary.

Thus the dihedral angle in the diagram may be designated by $P-A B-Q$.

When no confusion can arise it will be sufficient in speaking of a dihedral angle to name its edge.

427. Two planes which extend both ways from their line of intersection form, in all, four dihedral angles having the same edge, those being adjacent angles which have one boundary in common, and those being vertical angles in which the boundaries of one are the extensions of the boundaries of the other.

Thus in the diagram $P-A B-Q$ and $P-A B-R$

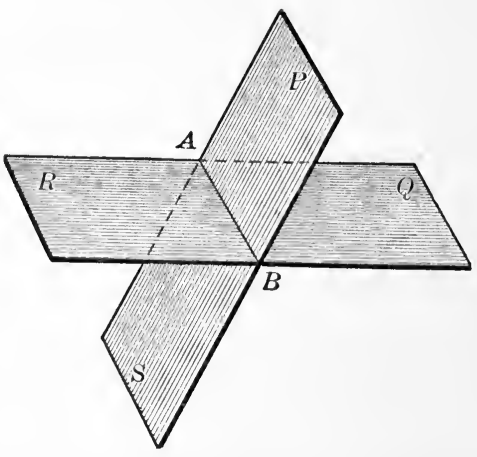
are adjacent angles, $P-A B-Q$ and $R-A B-S$ are vertical angles. 
428. Two dihedral angles are equal when their boundaries can be made to coincide.

429. Definition. If one plane meets another so as to make the adjacent dihedral angles equal, the first plane is said to be perpendicular to the second, and the angles so formed are called right dihedral angles.

430. If we choose a point in the edge of a dihedral angle, and from it draw two straight lines at random, one in each boundary of the dihedral angle, the plane angle formed by these lines may have any magnitude from zero to two right angles, the dihedral angle remaining unaltered.

How must the two lines be drawn in order that the angle between them shall be (1) zero? (2) two right angles ?

Definition. If from any point of the edge of a dihedral angle, two straight lines are drawn perpendicular to that edge, one in each boundary of the angle, the plane

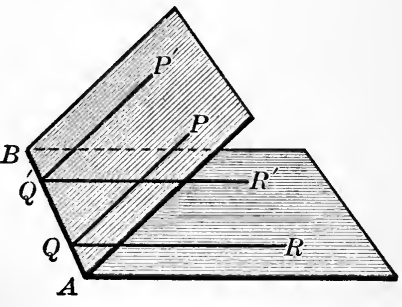
angle formed by these lines is called the inclination of the planes forming the dihedral angle; or it is called the plane angle of the dihedral angle.

Thus in the diagram $\angle P Q R$ is the inclination of the planes $P$ and $R$, if $P Q$ and $R Q$ are both perpendicular to the edge $A B$.

431. That the inclination of the planes is the same no matter from what point of the edge the perpendiculars are drawn follows from Proposition XIII. For since $Q P$ and $Q^{\prime} P^{\prime}$ lie in the same plane and are both perpendicular to $A B$, they are parallel (Art. 91). Similarly $Q R$ and $Q^{\prime} R^{\prime}$ are parallel. Therefore $\angle P Q R$ equals $\angle P^{\prime} Q^{\prime} R^{\prime}$ wherever on the edge $Q$ may be chosen.

In other words,

All plane angles of the same dihedral angle are equal. 
432. From the definition of the plane angle of a dihedral angle it follows that,

The lines of intersection with the boundaries of a dihedral angle, of any plane perpendicular to the edge of that angle, form the plane angle of the dihedral angle.

\section{Proposition XV}

433. Two dihedral angles are equal if their plane angles are equal.
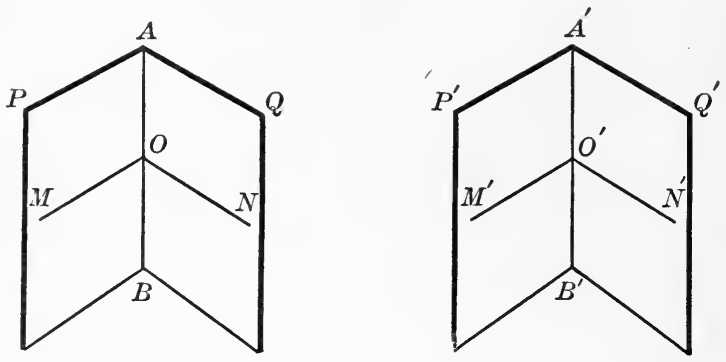

Let $P-A B-Q$ and $P^{\prime}-A^{\prime} B^{\prime}-Q^{\prime}$ be two dihedral angles whose plane angles, $M O N$ and $M^{\prime} O^{\prime} N^{\prime}$, are equal.

It is required to prove that the dihedral angles are equal.

Proof. Place the one figure upon the other so that $\angle M^{\prime} O^{\prime} N^{\prime}$ coincides with its equal $\angle M O N$.

The edge $A^{\prime} B^{\prime}$ must then coincide with the edge $A B$, since both are perpendicular to the plane $M O N$ at the point $O$.

(Art. 404.)

The plane $P^{\prime}$ must coincide with the plane $P$, since two lines determining $P^{\prime}$, viz:, $A^{\prime} B^{\prime}$ and $O^{\prime} M^{\prime}$, coincide with two lines determining $P$, viz., $A B$ and $O M$.

Similarly the plane $Q^{\prime}$ must coincide with the plane $Q$. Therefore the two dihedral angles, having been made to coincide, are equal.

\section{EXERCISE}

1. State and prove the converse of the above theorem. 


\section{Proposition XVI}

434. Two dihedral angles are in the same ratio as their plane angles.

Outline of proof.

CASE I. When the plane angles are commensurable.

1. Choose a common measure for the plane angles, and divide them both into equal parts. The numbers of parts will give the ratio of the plane angles.

2. Through the edges of the two dihedral angles and the lines of division of the plane angles pass planes. These will divide the dihedral angles into equal parts, the same in number as the equal parts of the plane angles.

3. The ratio of the dihedral angles will be given by the numbers of parts into which they are divided. This ratio will be the same as the ratio of the plane angles.

CASE II. When the plane angles are incommensurable.

1. Choose a measure of one of the plane angles; with it as unit divide that angle into equal parts and apply the unit as often as possible to the other plane angle.

2. Proceed with the proof as in Case II, Prop. X, Chap. III, applying the Principle of Limits. Give the full demonstration.

435. Note. Since dihedral angles are in the same ratio as their plane angles, the measure of the plane angle gives the measure of the dihedral angle. Or we may say that to measure a dihedral angle, we measure its plane angle. Thus we have a dihedral angle of $90^{\circ}$, or $30^{\circ}$, or $10^{\circ}$, when its plane angle is $90^{\circ}$, or $30^{\circ}$, or $10^{\circ}$.

A right dihedral angle has its plane angle equal to a right angle; and two planes are thus perpendicular to each other, when the plane angle of any one of the dihedral angles formed by them, is a right angle. 


\section{Proposition XVII}

436. If a straight line is perpendicular to a given plane, every plane containing that line is perpendicular to the given plane.

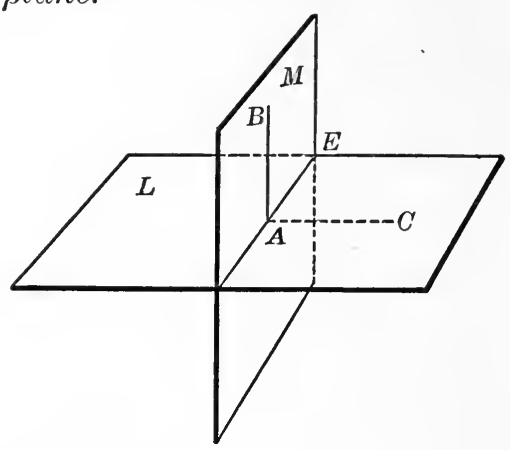

Let $A B$ be a straight line perpendicular to the given plane $L$, at the point $A$, and let $M$ be any plane containing $A B$.

It is required to prove that the plane $M$ is perpendicular to the plane $L$.

Proof. From $A$ draw the straight line $A C$ in the plane $I$, perpendicular to $A E$, the intersection of $L$ and $M$.

$\angle B A C$ is the plane angle of the dihedral angle formed by $L$ and $M$. Why?

$\angle B A C$ is a right angle. Why?

Therefore the plane $M$ is perpendicular to the plane $I$.

437. Corollary I. If a plane is perpendicular to any straight line of another plane, it is perpendicular to that other plane.

438. Corollary II. Any plane perpendicular to the edge of a dihedral angle is perpendicular to each of its faces.

\section{EXERCISES}

1. Through a given straight line pass a plane perpendicular to a given plane. 


\section{Proposition XVIII}

439. If two planes are perpendicular to each other, a straight line drawn in one of them, perpendicular to their intersection, is perpendicular to the other.

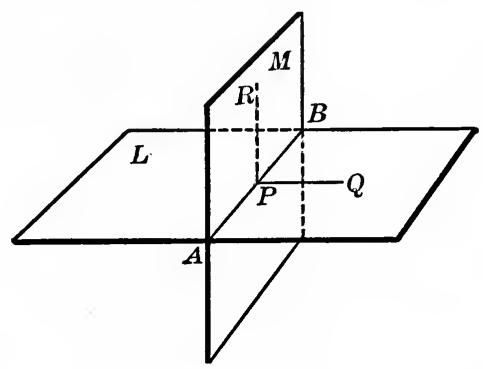

Let $L$ and $M$ be two planes perpendicular to each other, and let $P Q$ be drawn in the plane $L$ perpendicular to their intersection $A B$.

It is required to prove that $P Q$ is perpendicular to the plane $M$.

Proof. From $P$ draw $P R$ in the plane $M$ perpendicular to $P B$. $\angle R P Q$ is the plane angle of the dihedral angle formed by the planes $L$ and $M$, and is therefore a right angle by hypothesis.

Since $P Q$ is at right angles to both $P B$ and $P R$ it is perpendicular to the plane $\boldsymbol{M}$.

(Prop. IV.)

440. Conollary I. If two planes are perpendicular to each other, a straight line drawn from any point of their intersection, perpendicular to one plane, must lie in the other.

For if the line $P Q$ is drawn in $L$ perpendicular to the intersection of $L$ and $M$, it is perpendicular to $M$. Hence no other line drawn from $P$ can be perpendicular to $M$ (Art. 404). That is, the perpendicular to $M$, drawn from $P$, must lie in $L$. 
441. Corollary II. Every plane through a point, perpendicular to a given plane, contains the line from the point, perpendicular to the given plane.

442. Corollary III. If two planes are perpendicular to each other, a straight line drawn from any point in one of them, perpendicular to the other, must lie in the first plane.

For the line $Q P$, drawn from $Q$ perpendicular to the intersection of $L$ and $M$, lies in $L$, and is perpendicular to $M$. Hence, no other line drawn from $Q$ can be perpendicular to $M$.

(Art.409.)

That is, the perpendicular to $M$, drawn from any point of $L$, must lie in $L$.

443. Conollary IV. If two intersecting planes are each perpendicular to a third plane, their intersection is also perpendicular to that plane.

If $M$ and $N$ are each perpendicular to $L$, and $O$ is the point in which $L$ intersects the common line of $M$ and $N$, the perpendicular to $L$ drawn from $O$ must lie in both $M$ and $N$ (Cor. I). Hence it must be their intersection. That is, the intersection of $M$ and $N$ is perpendicular to $L$.

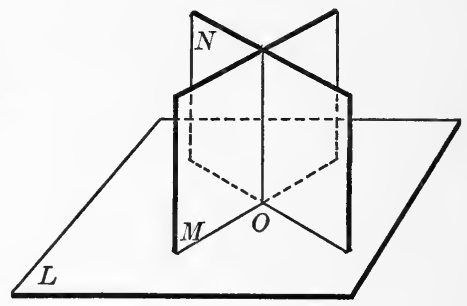

\section{EXERCISES}

1. Construct the plane which bisects a given dihedral angle.

2. Through a given point pass a plane perpendicular to each of two given planes.

3. Perpendiculars to a plane are drawn from the points of a straight line not lying in the plane. Show that the intersections of these perpendicular's with the plane are all in one straight line.

4. Through a point without a straight line any number of planes can pass parallel to that line.

5. Find the locus of points equidistant from two given parallel planes and at the same time equidistant from two given points. 


\section{Proposition XIX}

444. The locus of points equidistant from the boundaries of a dihedral angle is the plane bisecting that angle.

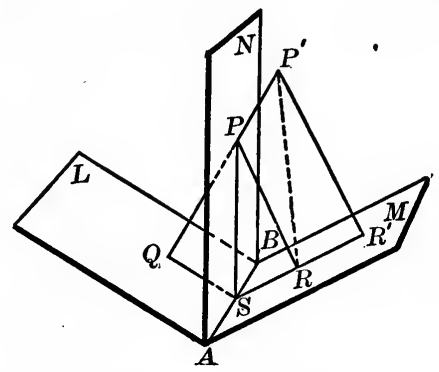

Let the planes $L$ and $M$ form a dihedral angle whose edge is $A B$, and let the plane $N$ bisect this angle.

It is required to prove that every point of $N$ is equidistant from $L$ and $M$, and that no point outside of $N$ is equidistant from $L$ and $M$.

Proof. First, in $N$ choose any point $P$, and from it draw $P Q$ and $P R$ perpendicular to $L$ and $M$, respectively. (Prop. VII.)

The plane of $P Q$ and $P R$ is perpendicular to both $L$ and $M$, and hence is perpendicular to the edge $A B$.

(Art. 443.)

Let this plane intersect $A B$ at $S$, and join $S P, S Q, S R$.

Then $\angle \mathrm{s} Q S R, Q S P$, and $P S R$ are the plane angles of the dihedral angles $L-A B-M, L-A B-N$, and $N-A B-M$, respectively.

Therefore $\angle Q S P=\angle P S R$ by hypothesis.

(Art. 432.)

Also $\angle \mathrm{s} P Q S$ and $P R S$ are right angles. Why?

Therefore $\triangle \mathrm{s} P Q S$ and $P R S$ are identically equal, and $P Q$ equals $P R$.

(Art. 106.)

Therefore $P$, any point of $N$, is equidistant from the planes $L$ and $M$.

Next, let $P^{\prime}$ be any point outside of the plane $N$, and from it 
draw perpendiculars $P^{\prime} Q$ and $P^{\prime} R^{\prime}$ to the planes $L$ and $M$, respectively. Let $P^{\prime} Q$ intersect the plane $N$ at the point $P$. Join $P^{\prime} R . \quad P^{\prime} R^{\prime}$ is less than $P^{\prime} R$. Why?

But $P^{\prime} R$ is less than $P^{\prime} P+P R$, i.e. less than $P^{\prime} Q$. Why? Therefore $P^{\prime} R^{\prime}$ is less than $P^{\prime} Q$.

Hence $P^{\prime}$ is not equidistant from $L$ and $M$.

Therefore the locus . . .

\section{Definitions}

445. The projection of a point upon a plane is the foot of the perpendicular drawn from the point to the plane.

The projection of a line upon a plane is the locus of the projections of its points upon the plane.

446. Theorem. The projection of a straight line upon a plane must be a straight line.

For, if $A$ be any point of the given line, and $P$ its projection upon the given plane, $A P$ and the given line determine a plane perpendicular to the given plane, in which lie the perpendiculars from all the points of the given line. (Art. 442.)

The projection of a curved line upon a plane is in general another curved line. Might it in any case be a straight line ?

447. The angle which a straight line makes with its projection on a plane is called the inclination of the line to the plane.

Whenever we speak of 'the angle which a straight line makes with a plane,' it is always the angle which it makes with its projection on the plane that is referred to.

448. Properly speaking, two straight lines form an angle only when they intersect. For convenience, however, we sometimes speak of the angle between two non-intersecting straight lines, or of the inclination of one straight line to another which does not meet it.

By the angle between two non-intersecting straight lines is meant the angle formed by two intersecting straight lines which are parallel to them. 


\section{Proposition XX}

449. The acute angle which a straight line makes with its own projection upon a plane is the least angle it makes with any line of that plane.

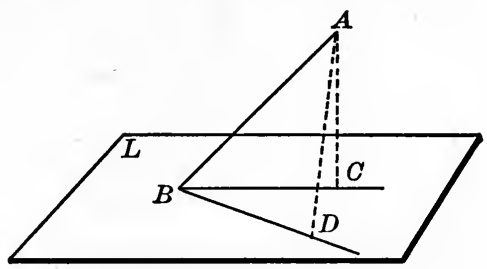

Let $A B$ be any straight line oblique to the plane $L$, and let $B C$ be its projection upon $L$.

It is required to prove that $\angle A B C$ is less than the angle which $A B$ makes with any other line of the plane.

Proof. From any point $A$ of the line $A B$ draw the perpendicular to the plane, meeting $B C$ at $C$.

From $B$ draw any other straight line $B D$ in the plane, making the segment $B D$ equal to $B C$.

Join $A D$.

Then $A D$ is greater than $A C$. Why?

Therefore $\angle A B D$ is greater than $\angle A B C$.

(Art. 80.)

That is, $\angle A B C$ is less than the angle which $A B$ makes with any other line of the plane.

\section{EXERCISES}

1. The projection of any straight line upon a given plane lies in a plane through the given line, perpendicular to the given plane.

2. If a straight line is parallel to a given plane, the projection of the line upon the plane is parallel to the line.

3. Parallel lines intersecting the same plane make equal angles with it. 


\section{Proposition XXI}

450. To draw a straight line which shall be perpendicular to each of two given straight lines not lying in the same plane.

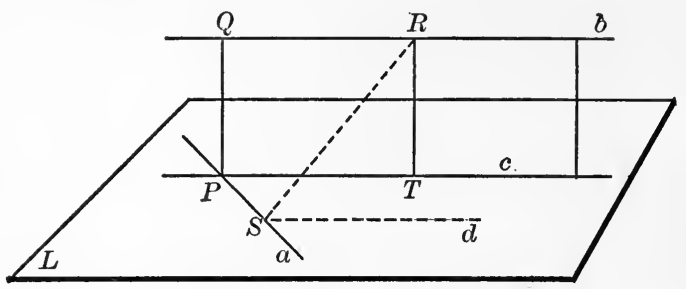

Let $a$ and $b$ be two straight lines not lying in the same plane.

It is required to find a straight line which shall be perpendicular to both $a$ and $b$.

Construction. Let $L$ be the plane through $a$ which is parallel to $b$.

(Art. 414.)

Also let $c$ be the projection of $b$ upon the plane $L$.

Then $c$ is parallel to $b$, and the plane of $b$ and $c$ is perpendicular to $L$. Why?

The straight lines $a$ and $c$ cannot be parallel, for then $a$ and $b$ must be parallel, contrary to hypothesis.

(Art. 396.)

Let $a$ and $c$ intersect at $P$, and from $P$ draw $P Q$ perpendicular to the plane $L$.

Then $P Q$ is the line required.

Proof. Since $P Q$ was drawn perpendicular to the plane $L$, at the point $P$, it is perpendicular to the line $a$, and lies in the plane of $b$ and $c$.

(Art. 440.)

And since $P Q$ is perpendicular to the line $c$ [why?], it is also perpendicular to $b$, meeting it, say, at the point $Q$.

Therefore the straight line $P Q$, meeting both $a$ and $b$, is perpendicular to both. 
451. Theorem. Only one straight line can be drawn perpendicular to each of two given straight lines not lying in the same plane.

If possible let $R S$ be a second straight line perpendicular to both $a$ and $b$.

Let the plane of $b$ and $R S$ intersect the plane $L$ in the line $d$.

Then $b$ is parallel to $d$ (Prop. $\mathrm{X}$ ), and $R S$ being perpendicular to $b$ is also perpendicular to $d$.

Now $R S$ was assumed perpendicular to $a$.

Therefore $R S$ is perpendicular to the plane $L$. (Prop. IV.)

But $R T$ drawn in the plane of $b$ and $c$, perpendicular to $c$, is perpendicular to $L$.

(Prop. XVIII.)

Thus we have two perpendiculars from $R$ to the plane $L$, which is impossible.

Therefore $R S$ cannot be perpendicular to both $a$ and $b$, and similarly no other line than $P Q$ can be perpendicular to both.

452. Theorem. The common perpendicular to two given straight lines not lying in the same plane, is the shortest line between them.

The shortest line from a point of $a$ to the line $b$ is perpendicular to $b$, and the shortest line from a point of $b$ to the line $a$ is perpendicular to $a$.

Therefore the shortest line from a point of $a$ to a point of $b$ must be perpendicular to both $a$ and $b$.

\section{EXERCISES}

1. Construct a plane parallel to each of two given non-intersecting straight lines and equidistant from them.

2. Draw a straight line to intersect three given non-intersecting straight lines at $P, Q, R$, respectively, so that $P Q$ shall equal $Q R$.

3. If the points of intersection of four parallel straight lines with any plane form the vertices of a parallelogram, their points of intersection with every plane form the vertices of a parallelogram. 


\section{SECTION III \\ POLYHEDRAL ANGLES}

453. Definition. Three planes meeting at a point form what is called a trihedral angle.

The planes are the boundaries, or the faces, of the angle; the point is the vertex of the angle; and the straight lines in which the planes intersect, two and two, are the edges of the angle.

In general, the figure formed by several planes meeting at a point is called a polyhedral angle. The planes intersect, two and two, in a certain order to form the edges.

A polyhedral angle may be designated by naming the vertex and another point in each edge in order. Thus the polyhedral angle in the diagram may be designated $A-B C D E$.

A polyhedral angle of four faces is called a tetrahedral angle.

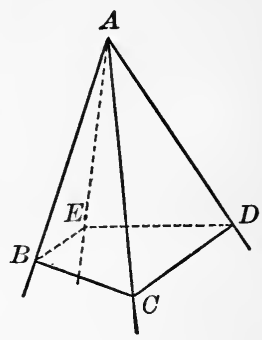

A polyhedral angle, or as it may be called, a solid angle, is a geometrical magnitude of a character essentially different from a plane angle. Like a plane angle, however, its magnitude depends, not upon the length of its edges, but only upon the amount of their divergence.

454. In a polyhedral angle the pairs of adjacent faces form dihedral angles, and the two edges lying in any face form a plane angle.

These are called respectively the dihedral angles and the face angles of the polyhedral angle.

The dihedral angles and the face angles are called the parts of a polyhedral angle.

A polyhedral angle is convex when the section of its faces made by any plane is a convex polygon.

455. Two polyhedral angles are identically equal when they can be made to coincide, each face with its corresponding face and each edge with its corresponding edge. 
456. Two polyhedral angles are symmetrical when the parts of the one are equal respectively to the parts of the other, but are arranged in reverse order.

Suppose the edges of any polyhedral angle $O-A B C D$ to be extended through the vertex, to points $A^{\prime}, B^{\prime}, C^{\prime}, D^{\prime}$.

The polyhedral angle $O-A^{\prime} B^{\prime} C^{\prime} D^{\prime}$ so formed is symmetrical to the angle $O-A B C D$. For the face angles and the dihedral angles are equal, each to

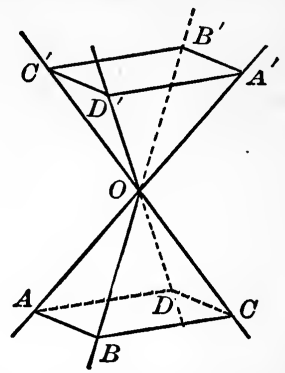
each, being vertically opposite in each case, but the edges are arranged in one order for the lower angle, and in the opposite order for the upper angle.

This will readily be seen if you imagine the eye placed at $O$, and look out along the edges in order, first for the lower angle, and then for the upper.

457. Two symmetrical polyhedral angles cannot in general be made to coincide.

Just as two triangles $A B C$ and $A^{\prime} B^{\prime} C^{\prime}$ lying in the same plane may be identically equal, but cannot be made to coincide without turning one of them over, so two symmetrical polyhedral angles can in general be made to coincide only by turning one of them
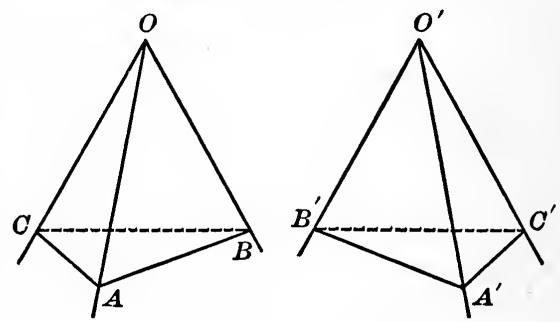
inside out, so to speak, by drawing the vertex down through the plane section.

In the same way one of your hands is symmetrical to the other, not identically equal to it. The one hand cannot be made to occupy exactly the same space as the other, and the left glove will fit the right hand only when it has been turned inside out. 


\section{Proposition XXII}

458. The sum of any two face angles of a trihedral angle is greater than the third face angle.

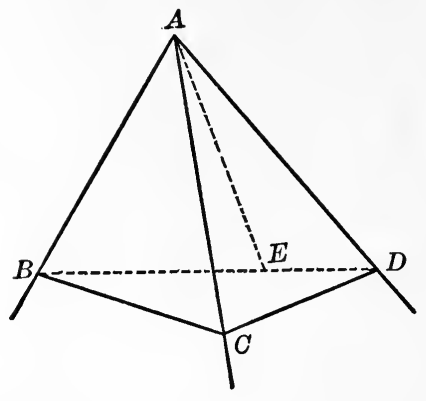

Let $A-B C D$ be any trihedral angle in which the face angle $B A D$ is greater than either of the other two.

It is required to prove that the sum of the face angles $B A C$ and $C A D$ is greater than the face angle $B A D$.

Proof. In the face $B A D$ draw the straight line $A E$ making $\angle B A E$ equal to $\angle B A C$.

Make the line-segments $A E$ and $A C$ equal, and through $C$ and $E$ pass a plane cutting the other two edges at $B$ and $D$.

Then

$$
B E=B C \text {. }
$$

Therefore $C D$ is greater than $E D$, since $B C$ and $C D$ are together greater than $B D$.

In $\triangle \mathrm{s} C A D$ and $E A D, A C=A E, A D=A D$, while $C D$ is greater than $E D$.

Therefore $\angle C A D$ is greater than $\angle E A D$.

(Art. 80.)

Therefore the sum of $\angle \mathrm{s} B A C$ and $C A D$ is greater than $\angle B A D$.

459. Conollary. Any face angle of a polyhedral angle is less than the sum of the remaining face angles. 


\section{Proposition XXIII}

460. The sum of the face angles of any convex polyhedral angle is less than four right angles.

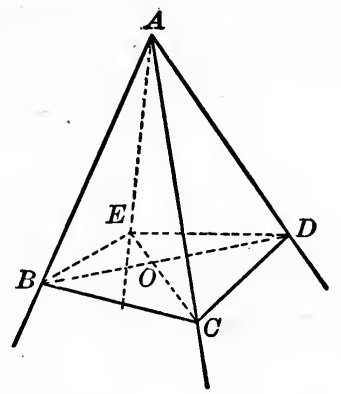

Let $A-B C D E$ be any convex polyhedral angle.

It is required to prove that the sum of the face angles $B A C$, $C A D, D A E$, etc., is less than four right angles.

Proof. Choose any plane cutting all the faces and edges of the given polyhedral angle. The section will be a convex polygon BCDE. Why?

The interior angles of the polygon $B C D E$, together with four right angles, make twice as many right angles as the polygon has sides.

(Art. 116.)

In any triangular face, as $\triangle B A C$, the sum of the angles is two right angles.

(Art. 101.)

Hence, the sum of all the angles in all the triangular faces equals twice as many right angles as the polyhedral angle has faces, i.e., twice as many right angles as the polygon $B C D E$ has sides.

The angles in all the triangular faces may be grouped as the face angles at the vertices, $B, C, D$, etc., together with the face angles at $A$.

Therefore, the face angles at $B, C, D$, etc., + the face angles at $A=$ the angles of the polygon $B C D E+$ four right angles, 
since each of these sums equals twice as many right angles as the polygon $B C D E$ has sides.

Now at any vertex, as $B$, the two face angles, $A B C$ and $A B E$, are together greater than the angle of the polygon $E B C$.

(Prop. XXII.)

Hence the sum of the face angles at all the vertices, $B, C, D$, etc., is greater than the sum of the angles of the polygon $B C D E$.

Therefore, the sum of the face angles at $A$ must be less than four right angles.

\section{Proposition XXIV}

461. If two trihedral angles have the three face angles of one equal, respectively, to the three face angles of the other, their corresponding dihedral angles are also equal.
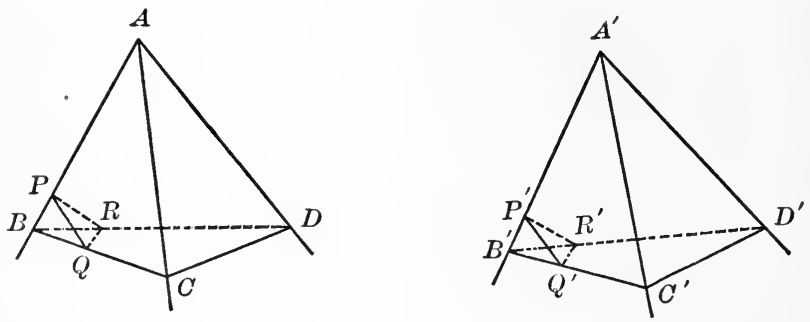

Let $A-B C D$ and $A^{\prime}-B^{\prime} C^{\prime} D^{\prime}$ be two trihedral angles having the face angles $B A C, C A D, D A B$ equal, respectively, to the face angles $B^{\prime} A^{\prime} C^{\prime}, C^{\prime} A^{\prime} D^{\prime}, D^{\prime} A^{\prime} B^{\prime}$.

It is required to prove that the dihedral angles whose edges are $A B, A C, A D$ are equal, respectively, to the dihedral angles whose edges are $A^{\prime} B^{\prime}, A^{\prime} C^{\prime}, A^{\prime} D^{\prime}$.

Proof. Let the planes $B C D$ and $B^{\prime} C^{\prime} D^{\prime}$ cut the edges of the two trihedral angles so as to make the segments $A B, A C, A D$, $A^{\prime} B^{\prime}, A^{\prime} C^{\prime}, A^{\prime} D^{\prime}$ all equal.

In $A B$ and $A^{\prime} B^{\prime}$ choose two points, $P$ and $P^{\prime}$, equidistant from $A$ and $A^{\prime}$, and through these points pass planes perpendicular to the edges $A B$ and $A^{\prime} B^{\prime}$. 
The plane through $P$ perpendicular to $A B$ will intersect $B C$ and $B D$ at some points $Q$ and $R$. Why?

Notice that $Q$ and $R$ are not necessarily within the segments $B C$ and $B D$.

Join $P Q, P R$, and $Q R$.

Similarly construct the triangle $P^{\prime} Q^{\prime} R^{\prime}$.

$\angle \mathrm{s} Q P R$ and $Q^{\prime} P^{\prime} R^{\prime}$ are the plane angles of the dihedral angles whose edges are $A B$ and $A^{\prime} B^{\prime}$ [why?], and it is required to prove them equal.

$\triangle \mathrm{s} A B C$ and $A^{\prime} B^{\prime} C^{\prime \prime}$ are identically equal isosceles triangles, by hy pothesis and construction.

$\triangle \mathrm{s} Q B P$ and $Q^{\prime} B^{\prime} P^{\prime}$ are identically equal.

(Art. 43.)

Therefore

$$
\begin{aligned}
& B Q=B^{\prime} Q^{\prime}, \\
& P Q=P^{\prime} Q^{\prime} .
\end{aligned}
$$

and

Similarly from $\triangle \mathrm{s} R B P$ and $R^{\prime} B^{\prime} P^{\prime}$,

$$
\begin{aligned}
& B R=B^{\prime} R^{\prime} . \\
& P R=P^{\prime} R^{\prime} .
\end{aligned}
$$

Now $\triangle \mathrm{s} B C D$ and $B^{\prime} C^{\prime} D^{\prime}$ are identically equal.

(Art. 53.)

Point out all the steps necessary to show this.

Therefore

$$
\text { - } \angle C^{\prime} B D=\angle C^{\prime} B^{\prime} D^{\prime} \text {. }
$$

Therefore $\triangle \mathrm{s} Q B R$ and $Q^{\prime} B^{\prime} R^{\prime}$ are identically equal (Art.41), and

$$
Q R=Q^{\prime} R^{\prime} .
$$

Therefore $\triangle \mathrm{s} Q P R$ and $Q^{\prime} P^{\prime} R^{\prime}$ are identically equal (Art.53), and

$$
\angle Q P R=\angle Q^{\prime} P^{\prime} R^{\prime} .
$$

That is, the dihedral angle whose edge is $A B$ equals the dihedral angle whose edge is $A^{\prime} B^{\prime}$.

Similarly it may be shown that the other dihedral angles are equal. 
Note. If the parts of the trihedral angles $A-B C D$ and $A^{\prime}-B^{\prime} C^{\prime} D^{\prime}$ are arranged in the same order around $A$, the trihedral angles are equal, and if in the reverse order, they are symmetrical but not equal. In the first case, they could be superposed; in the second case, they could not be superposed.

'The pupil should try to superpose the one trihedral angle on the other, beginning with the vertices $A$ and $A^{\prime}$ and the equal face angles $B A C$ and $B^{\prime} A^{\prime} C^{\prime}$. Will the edges $A D$ and $A^{\prime} D^{\prime}$ then lie on the same side of this face?

If the edge $A B$ is made to coincide with its corresponding edge $A^{\prime} B^{\prime}$, and $A C$ with $A^{\prime} C^{\prime}$, how will $A D$ and $A^{\prime} D^{\prime}$ lie?

If $A B$ is made to coincide with $A^{\prime} C^{\prime}$ and $A C$ with $A^{\prime} B^{\prime}$, how will $A D$ and $A^{\prime} D^{\prime}$ lie?

462. Definition. An isosceles trihedral angle is one in which two of the face angles are equal.

463. Theorem. An isosceles trihedral angle and its symmetrical trikedral angle are identically equal, i.e. can be superposed.

Suppose that $A-B C D$ is an isosceles trihedral angle having the face angles $B A C$ and $B A D$ equal; and suppose that $A^{\prime}-B^{\prime} C^{\prime} D^{\prime}$ is a trihedral angle having its face angles respectively equal to those of $A-B C D$, but arranged in reverse order.

The corresponding dihedral angles are equal by Proposition XXIV. Therefore the dihedral angle whose edge is $A B$ is equal
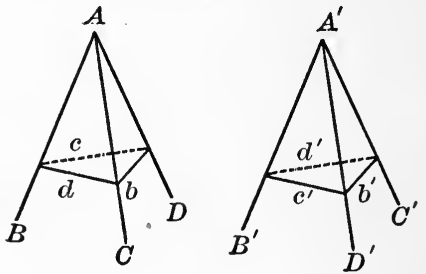
to the dihedral angle whose edge is $A^{\prime} B^{\prime}$.

Place the trihedral angle $A^{\prime}-B^{\prime} C^{\prime} D^{\prime}$ upon the trihedral angle $A-B C D$ so that the dihedral angle $A^{\prime} B^{\prime}$ coincides with the dihedral angle $A B$, the vertex $A^{\prime}$ coinciding with the vertex $A$.

Then since $\angle B^{\prime} A^{\prime} D^{\prime}=\angle B A D=\angle B A C$, by hypothesis, the edge $A^{\prime} D^{\prime}$ will coincide with the edge $A C$.

Similarly, the edge $A^{\prime} C^{\prime}$ will coincide with the edge $A D$, and the two trihedral angles will coincide in all their parts. 
Why could not two symmetrical trihedral angles whose face angles are all unequal be superposed in the same way? At what point would the process of superposition fail?

464. Conollary I. If two face angles of a trihedral angle are equal, the dihedral angles opposite them are also equal.

For, the trihedral angle and its symmetrical trihedral angle can be superposed.

465. Corollary II. If two isosceles trihedral angles have the three face angles of one equal, respectively, to the three face angles of the other, they are identically equal.

They can be superposed whether their parts are arranged in the same order or in opposite orders.

466. Theorem. If two trihedral angles have two face angles and the included dihedral angle of one equal, respectively, to two face angles and the included dihedral angle of the other, they are either identically equal or symmetrical, according as the parts are arranged in the same order or in opposite orders.

If the parts are arranged in the same order, the two ean be superposed ; if arranged in opposite orders, one can be superposed to the symmetric of the other.

467. Theorem. If two trihedral angles have a face angle and the two adjacent dihedral angles of one equal, respectively, to a face angle and the two adjacent dihedral angles of the other, they are either identically equal or symmetrical, according as the parts are arranged in the same order or in opposite orders.

\section{EXERCISES}

1. The planes that bisect the dihedral angles of any trihedral angle intersect in the same straight line.

2. If the face angle $A O B$ of the trihedral angle $O-A B C$ is bisected by the straight line $O D$, the angle $C O D$ is less than half the sum of the face angles $A O C$ and $B O C$. 


\section{MISCELLANEOUS EXERCISES}

1. From a point $A$ in one of two intersecting planes, $A B$ is drawn perpendicular to the first plane, meeting the second plane at $B$, and $A C$ is drawn perpendicular to the second plane, meeting it at $C$. Show that $B C$ is perpendicular to the line of intersection of the two planes.

2. Two line segments $A B$ and $D C$ are such that if $B C$ and $A D$ are joined each of the angles $A, B, C, D$, is a right angle. Prove that $A B$ and $D C$ are parallel.

3. If two triangles $A B C$ and $A^{\prime} B^{\prime} C^{\prime}$ in different planes are so situated that the lines $A A^{\prime}, B B^{\prime}, C C^{\prime}$ meet in a point $S$, then the pairs of corresponding sides $A B, A^{\prime} B^{\prime} ; A C, A^{\prime} C^{\prime} ; B C, B^{\prime} C^{\prime}$ intersect in points of one straight line.

Suggestion. Let the planes of $A B C$ and $A^{\prime} B^{\prime} C^{\prime}$ intersect in a straight line $s$. The points $S, A, A^{\prime}, B, B^{\prime}$ lie in one plane intersecting $s$ at $X$, say. $A B$ and $A^{\prime} B^{\prime}$ must intersect at $X$. Similarly for the other pairs.

4. Show that the locus of a point equidistant from three given points is a straight line through the centre of the circle determined by the three points.

5. Prove that if $A P$ makes equal angles with $A B$ and $A C$, it must lie in one or other of two fixed planes.

6. Of three given planes each is perpendicular to the other two. Show that of their three lines of intersection each is perpendicular to the other two.

7. If two intersecting planes are cut by two parallel planes, the lines of section of the first two by each of the parallel planes will make equal angles.

8. If a straight line is equally inclined to two given planes, show that it is perpendicular to the plane bisecting one of the dihedral angles formed by the two planes.

9. Show that the locus of the foot of the perpendiculars drawn from a fixed point to the planes through a fixed straight line is a circle.

10. Draw through the vertex of a trihedral angle a straight line which will make equal angles with the three edges of the trihedral angle. How many such lines are there?

11. The projections an a plane of two equal and parallel line-segments are equal and parallel. 


\section{SUMMARY OF CHAPTER VI}

\section{Definitions.}

(1) Solid Figure - one whose points and lines do not all lie in the same plane. $\$ 386$.

(2) Line Parallel to a Plane - a straight line which does not meet the plane, however far they may be extended. $\$ 392$.

(3) Line Perpendicular to a Plane - a straight line which is perpendicular to every line of the plane passing through their intersection. $\$ 393$.

(4) Line Oblique to a Plane - a straight line neither parallel nor perpendicular to the plane. $\$ 393$.

(5) Parallel Planes-planes which do not meet, however far they may be extended. §393.

(6) Skew or Gauche Lines - straight lines so situated that no plane can contain them both. Ex. 3, p. 271.

(7) Distance from a Point to a Plane - the length of the perpendicular from the point to the plane. $\$ 411$.

(8) Dihedral Angle - the figure formed by two planes meeting in a straight line. $\$ 426$.

(9) Right Dihedral Angle - an angle formed by two intersecting planes when the adjacent angles so formed are equal. $§ 429$.

(10) Plane Angle of a Dihedral Angle - the angle between two straight lines drawn perpendicular to the edge from the same point, one in each boundary. $\$ 430$.

(11) Projection of a Point upon a Plane - the foot of the perpendicular drawn from the point to the plane. $\$ 445$.

(12) Projection of a Line upon a Plane - the locus of the projections of its points upon the plane. $\S 445$.

(13) Inclination of a Line to a Plane - the angle between the line and its projection upon the plane. $\$ 447$.

(14) Trihedral Angle - the figure formed by three planes meeting at a point. $\$ 453$.

(15) Polyhedral Angle - the figure formed by several planes meeting at a point. $\$ 453$.

(16) Identically Equal Polyhedral Angles - two which can be made to coincide. $\$ 455$.

(17) Symmetrical Polyhedral Angles - two whose parts are equal, respectively, but arranged in reverse order. $\$ 456$.

(18) Isosceles Trihedral Angle - one having two face angles equal. $\S 462$. 
2. Postulate.

(1) Through three points not in the same straight line there can pass one and only one plane (Postulate 9). $\$ 388$.

3. Elemexts which deternine a Plane.

(1) Three points (Postulate 9). $\$ 388$.

(2) A straight line and a point not lying on it. $§ 388$.

(3) Two intersecting straight lines. $\$ 388$.

(4) Two parallel straight lines. $\$ 388$.

4. Problems.

(1) At a given point in a given plane to erect a perpendicular to the plane. $\$ 403$.

(2) From a given point without a given plane to draw a perpendicular to the plane. $\S 408$.

(3) To draw a straight line which shall be perpendicular to each of two given straight lines not lying in the same plane. $§ 450$.

5. Tileorems on the Intersections of Planes.

(1) The intersection of two planes is a straight line. $\$ 390$.

(2) If two planes have one point in common, they must have a second point, and hence a straight line, in cominon. \$391.

(3) If three planes intersect, two and two, their three lines of intersection are either concurrent, or are parallel, two and two. $\S 394$.

(4) The two planes determined by two given parallel lines and a point not lying in their plane, intersect in a line parallel to each of the given lines. $\$ 395$.

(5) Two parallel planes are intersected by any third plane in parallel lines. $§ 419$.

6. Theorems on Straight Lines perpendicular to Planes.

(1) If a straight line is perpendicular to each of two given straight lines at their point of intersection, it is perpendicular to the plane of these lines. $\$ 397$.

(2) At any point of a straight line one plane can be constructed perpendicular to that line, and only one. $\$ 398$.

(3) Through a given point not on a given straight line, one plane and only one can be constructed perpendicular to the given line. $\S 399$.

(4) Two intersecting planes cannot both be perpendicular to the same straight line. $§ 400$. 
(5) All the straight lines perpendicular to a given line at a given point lie in one plane perpendicular to the given line. $\$ 401$.

(6) If a plane bisects a given line-segment perpendicularly, every point of the plane is equidistant from the extremities of the line-segment, and conversely. $\$ 402$.

(7) At a point in a plane but one straight line can be drawn perpendicular to the plane. $\$ 404$.

(8) From a point without a plane only one perpendicular to the plane can be drawn. $\$ 409$.

(9) Two straight lines perpendicular to the same plane are parallel. $\S 405$.

(10) If one of two parallel lines is perpendicular to a plane, the other is also. $\S 407$.

(11) Two planes perpendicular to the same straight line are parallel. $\S 412$.

(12) A straight line perpendicular to one of two parallel planes is also perpendicular to the other. $\S 422$.

(13) If a straight line is perpendicular to a given plane, every plane containing that line is perpendicular to the given plane. $\S 436$.

7. Theorems on Straight Lines and Planes parallel to them.

(1) If two straight lines are parallel, any plane containing one of them, and not the other, is parallel to the other. $\S 413$.

(2) Through either of two given straight lines not lying in the same plane, one plane can be passed parallel to the other line. $\S 414$.

(3) Through a given point a plane can be passed parallel to any two given straight lines in space. $§ 415$.

(4) If a straight line is parallel to a given plane, it is parallel to the intersection of any plane through it, with the given plane. $\S 416$.

(5) If a straight line is parallel to a given plane, a line drawn from any point in the plane parallel to the given line lies in the given plane. $\$ 417$.

(6) If two intersecting straight lines are each parallel to a given plane, the plane determined by these lines is also parallel to the given plane. § 418 .

8. Theorems on Planes perpendicular to Each Other.

(1) If a straight line is perpendicular to a given plane, every plane containing that line is perpendicular to the given plane. $\$ 436$.

(2) Any plane perpendicular to the edge of a dihedral angle is perpendicular to each of its faces. $\S 438$. 
(3) If two planes are perpendicular to each other, a straight line drawn in one of them, perpendicular to their intersection, is perpendicular to the other. $\$ 439$.

(4) If two planes are perpendicular to each other, a straight line drawn from any point of their intersection, perpendicular to one plane, must lie in the other. $\$ 440$.

(5) If two planes are perpendicular to each other, a straight line drawn from any point in one of them, perpendicular to the other, must lie in the first plane. $\$ 442$.

(6) If two intersecting planes are each perpendicular to a third plane, their intersection is also perpendicular to that plane. $\S 443$.

9. Theorems on Parallel Planes.

(1) Two parallel planes are intersected by any third plane in parallel lines. $§ 419$.

(2) Parallel line-segments terminated by parallel planes are equal. $\S 420$.

(3) Two parallel planes are everywhere equidistant. $§ 421$.

(4) A straight line perpendicular to one of two parallel planes is also perpendicular to the other. $\$ 422$.

(5) If two straight lines are cut by three parallel planes, the corresponding segments are proportional. $\$ 425$.

10. Theorens ox Dimedral Angles.

(1) All plane angles of the same dihedral angle are equal. $\$ 431$.

(2) The lines of intersection with the boundaries of a dihedral angle, of any plane perpendicular to the edge of that angle, form the plane angle of the dihedral angle. $\S 432$.

(3) Two dihedral angles are equal if their plane angles are equal. $\S 433$.

(4) Two dihedral angles are in the same ratio as their plane angles. $\S 434$.

(5) The locus of points equidistant from the boundaries of a dihedral angle is the plane bisecting that angle. $\$ 444$.

11. Theorems on Trinedral and Polyiedral Angles.

(1) The sum of any two face angles of a trihedral angle is greater than the third face angle. $\$ 458$.

(2) Any face angle of a polyherlral angle is less than the sum of the remaining face angles. $§ 459$.

(3) The sum of the face angles of any convex polyhedral angle is less than four right angles. $\S 460$. 
(4) If two trihedral angles have the three face angles of one equal, respectively, to the three face angles of the other, their corresponding dihedral angles are also equal. $\S 461$.

(5) An isosceles trihedral angle and its symmetrical trihedral angle are identically equal. $\$ 463$.

(6) If two face angles of a trihedral angle are equal, the dihedral angles opposite them are also equal. $\S 464$.

(7) If two isosceles trihedral angles have the three face angles of one equal, respectively, to the three face angles of the other, they are identically equal. $\$ 465$.

(8) If two trihedral angles have two face angles and the included dihedral angle of one equal, respectively, to two face angles and the included dihedral angle of the other, they are either identically equal or symmetrical, according as the parts are arranged in the same order or in opposite orders. $\$ 466$.

(9) If two trihedral angles have a face angle and the two adjacent dihedral angles of one equal, respectively, to a face angle and the two adjacent dihedral angles of the other, they are either identically equal or symmetrical, according as the parts are arranged in the same order or in opposite orders. $\$ 467$.

\section{Miscellaneous Theorems.}

(1) Two straight lines each parallel to a third line are parallel to each other. $\S 396$.

(2) If from the foot of a given perpendicular to a plane, a straight line is drawn at right angles to any line of the plane, any line through their intersection which meets the given perpendicular is at right angles to the line of the plane. $\$ 406$.

(3) Of all straight lines which can be drawn from a point to a plane, the perpendicular is the shortest. $\$ 410$.

(4) If two intersecting straight lines lying in one plane are parallel, respectively, to two intersecting straight lines lying in another plane, the two planes must be parallel, and the angles formed by the lines are equal. $\S 424$.

(5) The acute angle which a straight line makes with its own projection upon a plane is the least angle it makes with any line of that plane. $\S 449$.

(6) Only one straight line can be drawn perpendicular to each of two given straight lines not lying in the same plane. § 451 .

(7) The common perpendicular to two given straight lines not lying in the same plane is the shortest line between them. $\S 452$. 


\title{
CHAPTER VII
}

\section{PRISMS AND PYRAMIDS}

\author{
$\longrightarrow+\infty 0500-$ \\ Section I \\ AREA AND VOLUME OF A PRISM \\ Definitions
}

468. A section of a surface made by an intersecting plane is the locus of points common to the surface and the plane.

469. A surface, such that every section of it made by an intersecting plane consists of one or more closed lines, is called a closed surface.

470. A closed surface which is made up wholly of intersecting planes is called a polyhedron.

The planes are called the faces of the polyhedron; the lines in which the faces intersect are called the edges; the points in which the edges
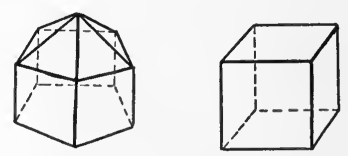
intersect are called the vertices.

The line-segment connecting any two vertices not lying in the same face is called a diagonal of the polyhedron.

If a polyhedron lies wholly on one side or the other of each of its faces, it is called a convex polyhedron.

Any section of a convex polyhedron made by a plane is a convex polygon. 
A polyhedron of four faces is called a tetrahedron; one of five faces is called a pentahedron; one of six faces is called a hexahedron; one of eight faces is called an octahedron; one of twelve faces is called a dodecahedron; one of twenty faces is called an icosahedron.

471. A polyhedron of which two faces are convex polygons lying in parallel planes and identically equal, while the remaining faces are parallelograms, is called a prism.

The equal parallel faces are called the bases of the prism; the remaining faces, the lateral faces. The edges lying in the bases are called the base
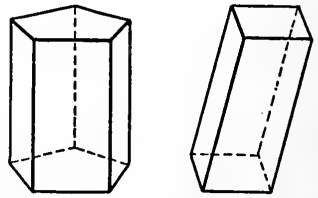
edges; and the intersections of the lateral faces, the lateral edges.

The base edges of a prism are equal and parallel, two and two, each edge of one base being equal and parallel to an edge of the other base.

The lateral edges of a prism are all equal and parallel (Art. 420), and make equal angles with the plane of either base.

A prism is called triangular, quadrilateral, etc., according as its bases are triangles, quadrilaterals, etc.

472. A right prism is one whose lateral edges are perpendicular to its bases.

If the lateral edges are not perpendicular to the bases, the prism is called oblique.

473. A right section of a prism is the section made by any plane perpendicular to the lateral edges.

474. The lateral area of a prism is the sum of the areas of the lateral faces of the prism.

475. The altitude of a prism is the perpendicular distance between its bases. 


\section{Proposition I}

476. The sections of a prism made by parallel planes are polygons which are identically equal.

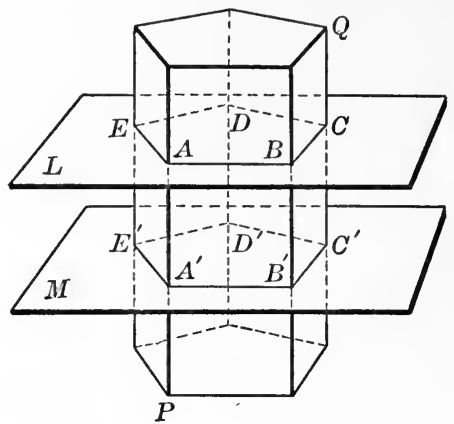

Let the sections of the prism $P Q$, made by the parallel planes $L$ and $M$, be $A B C D E$ and $A^{\prime} B^{\prime} C^{\prime} D^{\prime} E^{\prime}$.

It is required to prove that the polygons $A B C D E$ and $A^{\prime} B^{\prime} C^{\prime} D^{\prime} E^{\prime}$ are identically equal.

Proof. First, $A B$ is parallel to $A^{\prime} B^{\prime}$.

And since $A A^{\prime}$ is parallel to $B B^{\prime}$ by definition, $A A^{\prime} B^{\prime} B$ is a parallelogram. Therefore $A B=A^{\prime} B^{\prime}$.

Similarly, $B C=B^{\prime} C^{\prime}, C D=C^{\prime} D^{\prime}$, and so on.

Hence, $A B C D E$ and $A^{\prime} B^{\prime} C^{\prime} D^{\prime} E^{\prime}$ are mutually equilateral.

Next, since $A B$ is parallel to $A^{\prime} B^{\prime}$, and $B C$ is parallel to $B^{\prime} C^{\prime}$,

$$
\angle A B C=\angle A^{\prime} B^{\prime} C^{\prime} \text {. }
$$

Similarly, $\angle B C D=\angle B^{\prime} C^{\prime} D^{\prime}, \angle C D E=\angle C^{\prime} D^{\prime} E^{\prime}$, and so on. Hence, $A B C D E$ and $A^{\prime} B^{\prime} C^{\prime} D^{\prime} E^{\prime}$ are mutually equiangular. Therefore the polygons $A B C D E$ and $A^{\prime} B^{\prime} C^{\prime} D^{\prime} E^{\prime}$ are identically equal.

Note. The same proof applies if the planes of section intersect some or all of the edges produced. 
477. Conollary I. All right sections of the same prism are identically equal.

478. Corollary II. The section of a prism made by a plane parallel to either base is identically equal to the base.

\section{Proposition II}

479. The lateral area of a prism is equal to the product of a lateral edge and the perimeter of a right section of the prism.

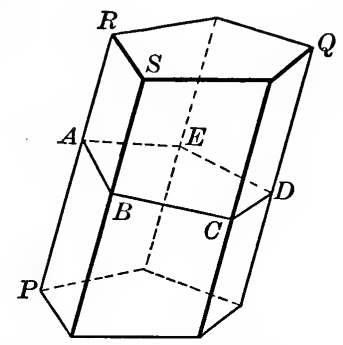

Let $A B C D E$ be a right section of the prism $P Q$, and $P R$ be any one of the lateral edges.

It is required to prove that the lateral area of $P Q$ is equal to the product of $P R$ and the perimeter of $A B C D E$.

Proof. $A B$ is perpendicular to $P R$. Why?

Therefore the area of the lateral face $P S$ is equal to the product of $P R$ and $A B$.

(Art. 305.)

Similarly, the area of each lateral face is equal to the product of a lateral edge $(=P R)$ and a side of $A B C D E$.

Therefore the lateral area of $P Q$ is equal to the product of $P R$ and $(A B+B C+C D+$ etc. $)$.

That is, the lateral area of $P Q$ is equal to the product of $P R$ and the perimeter of $A B C D E$.

480. Corollary. The lateral area of a right prism is equal to the product of its altitude and the perimeter of its base. 


\section{Proposition III}

481. Two prisms are identically equal if the three faces forming a trihedral angle in one are identically equal to the three faces forming a trihedral angle in the other and are similarly placed.
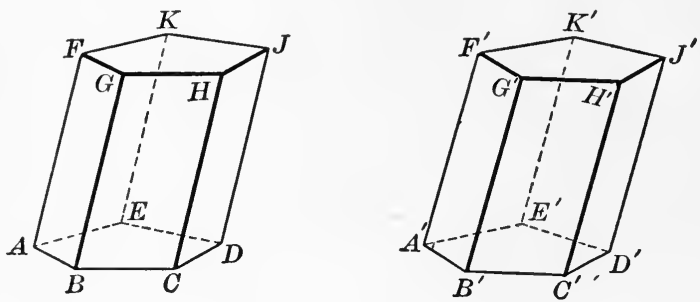

Let $A J$ and $A^{\prime} J^{\prime}$ be two prisms having the three faces $B E$, $B F$, and $B H$, forming the trihedral angle $B$, identically equal, respectively, to the three faces $B^{\prime} E^{\prime \prime}, B^{\prime} F^{\prime \prime}, B^{\prime} H^{\prime}$, forming the trihedral angle $B^{\prime}$, the equal faces being similarly placed.

It is required to prove that the two prisms $A J$ and $A^{\prime} J^{\prime}$ are identically equal.

Proof. First, the trihedral angles $B$ and $B^{\prime}$ are identically equal.

Apply the prism $A^{\prime} J^{\prime}$ to the prism $A J$ so that the trihedral angle $B^{\prime}$ coincides with the trihedral angle $B$.

Then, since the faces forming these angles are identically equal, two and two, the vertices $A^{\prime}, B^{\prime}, C^{\prime}, D^{\prime}, E^{\prime}, F^{\prime \prime}, G^{\prime}, H^{\prime}$, will coincide with the vertices $A, B, C, D, E, F, G, H$; and the edges connecting these vertices will also coincide.

Now, since the lateral edges of each prism are parallel and equal (Art. 471), and the edges $A^{\prime} F^{\prime \prime}, B^{\prime} G^{\prime}, C^{\prime} H^{\prime}$, coincide respectively with the edges $A F, B G, C H$, while the vertices $D^{\prime}, E^{\prime}$, coincide with $D, E$, therefore the remaining lateral edges $D^{\prime} J^{\prime}$ and $E^{\prime} K^{\prime}$ must coincide with the remaining edges 
$D J$ and $E K$, and the remaining vertices $J^{\prime}$ and $K^{\prime}$ with the remaining vertices $J$ and $K$.

Hence the two prisms coincide throughout.

482. Corollary I. Two right prisms are identically equal if their bases are identically equal and they have equal altitudes.

If the sides of the bases are not arranged in the same order, one of the prisms may be turned over, and then they can be made to coincide.

Could this be done if the prisms were not right prisms?

Why is it necessary to have the words 'and similarly placed' in the enunciation of the main theorem?

483. Definition. A truncated prism is a polyhedron having parallel lateral edges like a prism, but bases which are neither parallel nor equal.

The lateral faces cannot all be parallelograms, though some of them may be.

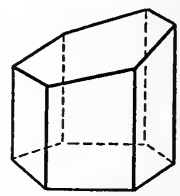

484. Theorem. Two truncated prisms are identically equal if the three faces forming a trihedral angle in one are identically equal to the three faces forming a trihedral angle in the other, and are similarly placed.

485. Definition. The space contained within a closed surface is called the volume of the figure.

The volume of a polyhedron is thus the space enclosed by its faces.

If two closed surfaces are placed side by side so as to have some portion in common, and this common portion is disregarded, the volume of the resulting figure is called the sum of the volumes of the two given figures.

486. Ахтом 13. If two figures are identically equal, their volumes are equal.

487. Ахгом 14. If equal volumes be added to equal volumes, or to the same volume, their sums are equal. 


\section{Proposition IV}

488. An oblique prism is equal in volume to a right prism whose base is a right section of the oblique prism and whose altitude is equal to a lateral edge of the oblique prism.

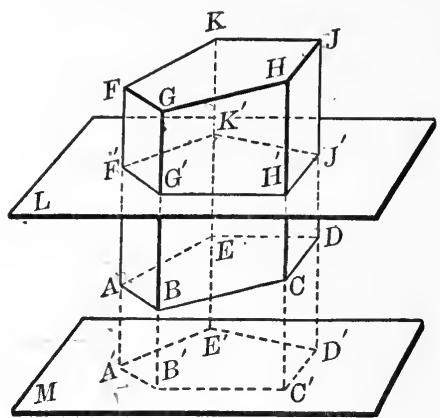

Let $A J$ be any oblique prism of which $F^{\prime \prime} G^{\prime} H^{\prime} J^{\prime} K^{\prime}$ is a right section made by the plane $L$.

It is required to prove that the prism $A J$ is equal in volume to a right prism whose base is $F^{\prime \prime} G^{\prime} H^{\prime} J^{\prime} K^{\prime}$, and whose altitude is equal to an edge of $A J$.

Proof. Produce the edge $F A$ to $A^{\prime}$ making $F^{\prime} A^{\prime}$ equal to $F A$.

Through $A^{\prime}$ pass a plane $M$ parallel to the plane $L$, and meeting the other lateral edges of the prism produced in $B^{\prime}, C^{\prime}, D^{\prime}, E^{\prime}$.

Then the figure $A^{\prime} \cdot J^{\prime}$ is a prism whose lateral edges are equal to the lateral edges of $A J$ and whose bases are right sections of $A J$.

Does $A^{\prime} J^{\prime}$ satisfy the definition of a prism?

The quadrilateral $A B B^{\prime} A^{\prime}$ is identically equal to the quadrilateral $F G G^{\prime} F^{\prime \prime}$. Why? 
Similarly, the quadrilateral $B C C^{\prime} B^{\prime}$ is identically equal to the quadrilateral $G H H^{\prime} G^{\prime}$.

And the polygon $A B C D E$ is identically equal to the polygon $F G H J K$, by hypothesis.

Therefore the truncated prism $A^{\prime} D$ is identically equal to the truncated prism $F^{\prime} J$, having the three faces forming the trihedral angle $B$ identically equal, two and two, to the three faces forming the trihedral angle $G$.

To each of these truncated prisms add the figure $A J^{\prime}$.

Then the volume of the right prism $A^{\prime} J^{\prime}$ is equal to the volume of the given prism $A J$.

(Axioms 13 and 14.)

Therefore ...

\section{Definitions}

489. A prism whose bases are parallelograms is called a parallelepiped.

A parallelepiped is thus a hexahedron, all of whose faces are parallelograms.

Lateral faces which contain opposite sides of the bases are called opposite faces.

490. A right parallelepiped is one in which one set of lateral edges is perpendicular to the bases.

491. A rectangular parallelepiped is a right parallelepiped whose bases are rectangles.

492. A cube is a parallelepiped whose faces are all squares.

\section{EXERCISES}

1. At least four of the faces of a right parallepiped are rectangles.

2. All the faces of a rectangular parallelepiped are rectangles.

3. If the three faces of a parallelepiped which meet in any vertex are squares, the figure is a cube. 


\section{Proposition V}

493. The opposite lateral faces of any parallelepiped are parallel and identically equal.

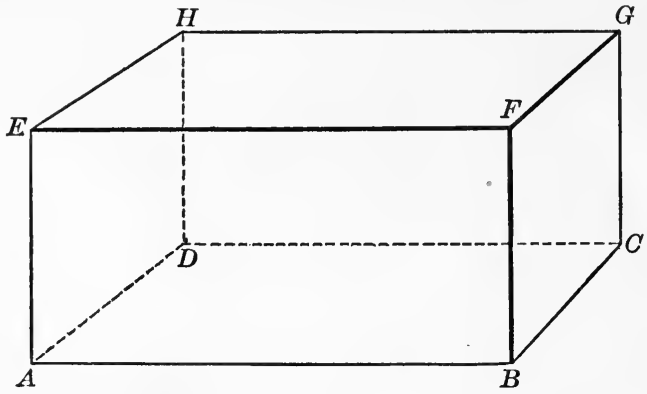

Let $A G$ be a parallelepiped whose bases are $A B C D$ and EFGH.

It is required to prove that the opposite lateral faces $A F$ and $D G$, also $A H$ and $B G$ are parallel and identically equal.

Proof. First, since $A B$ is parallel to $D C$, and $A E$ to $D H$, the lateral face $A F$ is parallel to $D G$.

Similarly, $A H$ is parallel to $B G$.

(Art. 424.)

Next, in the parallelograms $A F$ and $D G, A E=D H, A B=D C$, and $\angle E A B=\angle H D C$.

Therefore $A F$ is identically equal to $D G$.

(Art. 129.)

494. Coroliary. Any two opposite faces of a parallelepiped may be taken as the bases.

When any pair of opposite faces have been chosen as bases, the edges meeting them must be looked upon as the lateral edges.

\section{EXERCISES}

1. Any section of a parallelepiped cutting four edges between the vertices is a parallelogram.

2. Prove that in a rectangular parallelepiped each edge is perpendicular to all the edges meeting it. 


\section{Proposition VI}

495. The plane passed through two diagonally opposite edges of a parallelepiped divides it into two triangular prisms which are equal in volume.

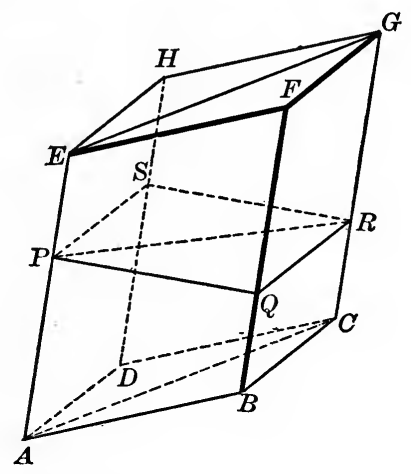

Let $B H$ be any parallelepiped, and let a plane be passed through the opposite edges $A E$ and $C G$.

It is required to prove that the triangular prism $A B C-F$ is equal in volume to the triangular prism $A D C-H$.

Proof. Take a right section $P Q R S$ of the parallelepiped, its plane intersecting the plane through the edges in the line $P R$.

Then $P Q R S$ is a parallelogram of which $P R$ is a diagonal.

The volume of $A B C-F$ is equal to the volume of a right prism having $\triangle P Q R$ for base and $A E$ for lateral edge.

(Prop. IV.)

Similarly, the volume of $A D C-H$ is equal to the volume of a right prism having $\triangle P S R$ for base and $A E$ for lateral edge.

But $\triangle P Q R$ is identically equal to $\triangle P S R$.

(Art. 124.)

Hence the right prism whose base is $\triangle P Q R$ and lateral edge $A E$ is identically equal to the right prism whose base is $\triangle P S R$ and lateral edge $A E$.

(Art. 482.)

Therefore the triangular prism $A B C-F$ is equal in volume to the triangular prism $A D C-H$. 


\section{Proposition VII}

496. The volumes of two rectangular parallelepipeds having identically equal bases are in the same ratio as their altitudes.
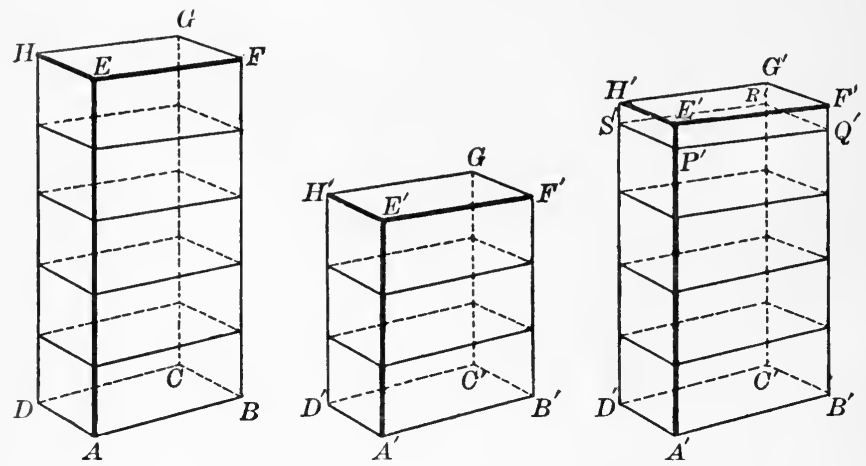

Let $A G$ and $A^{\prime} G^{\prime}$ be two rectangular parallelepipeds having their bases $A B C D$ and $A^{\prime} B^{\prime} C^{\prime} D^{\prime}$ identically equal.

It is required to prove that the volume of $A G$ is to the volume of $A^{\prime} G^{\prime}$ as the altitude $A E$ is to the altitude $A^{\prime} E^{\prime}$.

Proof. CASE I. When $A E$ and $A^{\prime} E^{\prime}$ are commensurable.

Choose unit length which will measure both $A E$ and $A^{\prime} E^{\prime}$.

Apply this unit length to the altitudes, and suppose that it divides $A E m$ times, and $A^{\prime} E^{\prime} n$ times.

Then

$$
A E: A^{\prime} E^{\prime}=m: n \text {. }
$$

Through all the points of division of $A E$ and $A^{\prime} E^{\prime}$ pass planes parallel to the bases.

These planes divide $A G$ into $m$ and $A^{\prime} G^{\prime}$ into $n$ right parallelepipeds, which are identically equal.

(Art. 482.)

Hence

Therefore

$$
\frac{\text { volume of } A G}{\text { volume of } A^{\prime} G^{\prime}}=\frac{m}{n} \text {. }
$$

$$
\frac{\text { volume of } A G}{\text { volume of } A^{\prime} G^{\prime}}=\frac{A E}{A^{\prime} E^{\prime}} \text {. }
$$


CAse II. When $A E$ and $A^{\prime} E^{\prime}$ are incommensurable.

Choose any unit length which will measure $A E$, and apply it as often as possible to $A^{\prime} E^{\prime}$.

Let $P^{\prime}$ be the last point of exact division so that $A^{\prime} P^{\prime}$ is a multiple of the unit length, and the remainder $P^{\prime} E^{\prime}$ is less than the unit length.

Through $P^{\prime}$ pass a plane $P^{\prime} Q^{\prime} R^{\prime} S^{\prime}$ parallel to the base.

Then $A^{\prime} R^{\prime}$ is a parallelepiped, and since $A E$ and $A^{\prime} P^{\prime}$ are commensurable,

$$
\frac{\text { volume of } A G}{\text { volume of } A^{\prime} R^{\prime}}=\frac{A E}{A^{\prime} P^{\prime}} \text {. }
$$

If now the length of the unit measure be repeatedly diminished, the remainder $P^{\prime} E^{\prime}$ can be made smaller than any assigned quantity, so that $A^{\prime} P^{\prime}$ approaches $A^{\prime} E^{\prime}$ as its limit, and the parallelepiped $A^{\prime} R^{\prime}$ approaches the parallelepiped $A^{\prime} G^{\prime}$ as its limit.

Also, $\frac{\text { volume of } A G}{\text { volume of } A^{\prime} R^{\prime}}$ will approach $\frac{\text { volume of } A G}{\text { volume of } A^{\prime} G^{\prime}}$ as its limit.
And
$\frac{A E}{A^{\prime} P^{\prime}}$ will approach $\frac{A E}{A^{\prime} E^{\prime}}$ as its limit.
But

$$
\frac{\text { volume of } A G}{\text { volume of } A^{\prime} R^{\prime}}=\frac{A E}{A^{\prime} P^{\prime}}
$$

for every unit of measure.

Therefore

$$
\frac{\text { volume of } A G}{\text { volume of } A^{\prime} G^{\prime}}=\frac{A E}{A^{\prime} E^{\prime}} \text {. }
$$

497. Definition. The lengths of the three edges which meet in any vertex are called the dimensions of a rectangular parallelepiped.

498. Theorem. If two rectangular parallelepipeds have two dimensions of one equal, respectively, to two dimensions of the other, their volumes are in the same ratio as their third dimensions.

This is merely a restatement of Proposition VII. 


\section{Proposition VIII}

499. The volumes of two rectangular parallelepipeds having equal altitudes are in the same ratio as the areas of their bases.
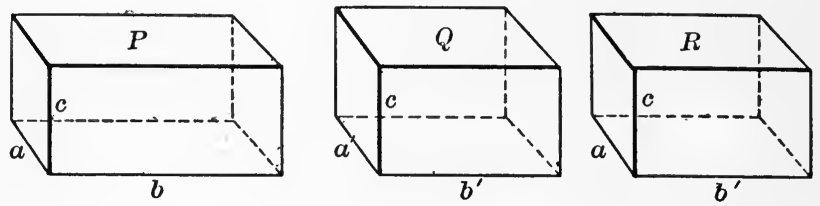

Let $P$ and $Q$ be the volumes of two rectangular parallelepipeds having equal altitudes $c$, and the dimensions of whose bases are $a, b$, and $a^{\prime}, b^{\prime}$.

It is required to prove that

$$
\frac{P}{Q}=\frac{a \times b}{a^{\prime} \times b^{\prime}} .
$$

Proof. Construct a third rectangular parallelepiped whose volume is $R$, and whose dimensions are $a, b^{\prime}, c$.

Then

$$
\frac{P}{R}=\frac{b}{b^{\prime}},
$$

also

$$
\frac{R}{Q}=\frac{a}{a^{\prime}} .
$$

Therefore

$$
\begin{aligned}
\frac{P}{R} \times \frac{R}{Q} & =\frac{b}{b^{\prime}} \times \frac{a}{a^{\prime}}, \\
\frac{P}{Q} & =\frac{a \times b}{a^{\prime} \times b^{\prime}} .
\end{aligned}
$$

500. TheoRem. If two rectangular parallelepipeds have one dimension of the one equal to one dimension of the other, their volumes are in the same ratio as the products of their other two dimensions.

This is again a restatement of Proposition VIII. 


\section{Proposition IX}

501 The volumes of any two rectangular parallelepipeds are in the same ratio as the products of their three dimensions.
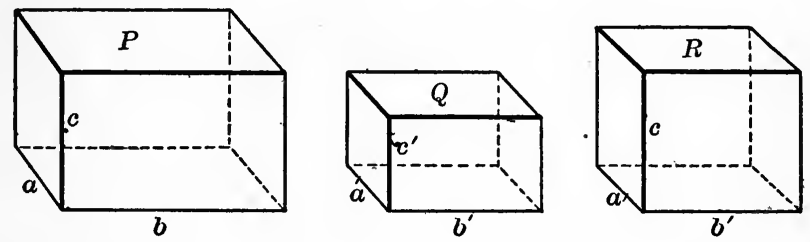

Let $P$ and $Q$ be the volumes of two rectangular parallelepipeds whose dimensions are $a, b, c$, and $a^{\prime}, b^{\prime}, c^{\prime}$, respectively.

It is required to prove that

$$
\frac{P}{Q}=\frac{a \times b \times c}{a^{\prime} \times b^{\prime} \times c^{\prime}} .
$$

Proof. Construct a third rectangular parallelepiped whose volume is $R$ and whose dimensions are $a^{\prime}, b^{\prime}, c$.

Then

$$
\frac{P}{R}=\frac{a \times b}{a^{\prime} \times b^{\prime}}
$$

and

$$
\frac{R}{Q}=\frac{c}{c^{\prime}} \text {. }
$$

Therefore

or

$$
\begin{aligned}
\frac{P}{R} \times \frac{R}{Q} & =\frac{a \times b}{a^{\prime} \times b^{\prime}} \cdot \frac{c}{c^{\prime}} \\
\frac{P}{Q} & =\frac{a \times b \times c}{a^{\prime} \times b^{\prime} \times c^{\prime}} .
\end{aligned}
$$

502. In order to express the measure of any volume it is first necessary to select a unit volume, with which the given volume is to be compared.

The unit volume usually chosen is the volume of a cube, each of whose edges is of unit length. 
When the unit of length is a foot, the unit of volume is the volume of a cube each of whose edges is a foot in length; or, as we say, the unit of volume is a cubic foot.

When the unit of length is an inch, or a yard, the unit of volume is a cubic inch, or a cubic yard.

The measure of a volume is the number which expresses how many times it will contain the unit of volume.

If in a rectangular parallelepiped, the

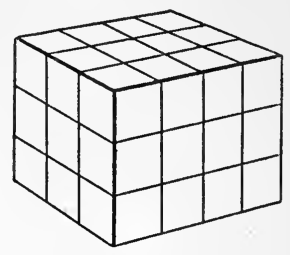
three edges which meet in any vertex are commensurable magnitudes, and these are divided into unit lengths, the whole volume can be divided into unit volumes by passing planes through the points of division. Thus the parallelepiped in the diagram contains thirty-six units of volume.

For brevity we frequently say "the volume of a parallelepiped" is a certain number, when it would be more exact to say "the measure of the volume."

In the preceding proposition, if the parallelepiped whose volume is $Q$ should have each edge of unit length, so that

$$
a^{\prime}=b^{\prime}=c^{\prime}=1,
$$

then $Q$ would be the unit volume.

In that case

or

$$
\frac{P}{Q}=a \times b \times c,
$$

$P=a \times b \times c$ times unit volume.

That is, $a \times b \times c$ is the measure of the volume $P$.

503. Theorem. The measure of the volume of any rectangular parallelepiped is the product of its three dimensions.

504. Corollary. The volume of any rectangular parallelepiped is the product of its altitude and the area of its base. 


\section{Proposition X}

505. The volume of any parallelepiped is equal to the product of its altitude and the area of its base.

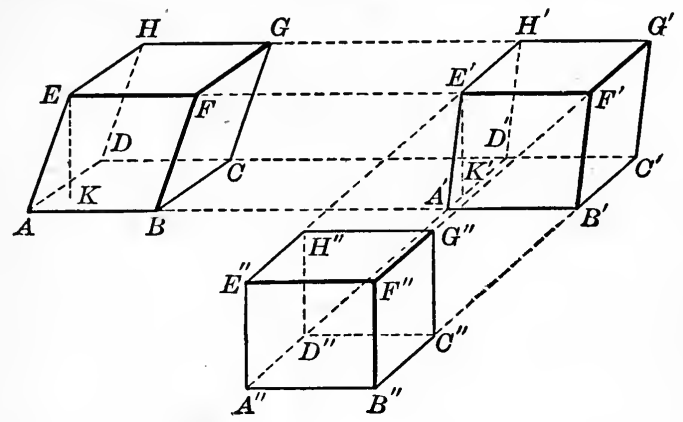

Let $A G$ be any oblique parallelepiped whose base is $A B C D$ and altitude $K E$.

It is required to prove that the volume of $A G$ is equal to the product of $K E$ and the area of $A B C D$.

Proof. Produce the edges $A B, D C, H G, E F$, and cut them perpendicularly at $A^{\prime}, D^{\prime}, H^{\prime}, E^{\prime}$, and $B,^{\prime} C,^{\prime} G^{\prime}, F^{\prime \prime}$, by two parallel planes whose distance apart $A^{\prime} B^{\prime}$ is equal to $A B$.

Then $A^{\prime} G^{\prime}$ is a right parallelepiped, $A^{\prime} H^{\prime}$ being the base, equal in volume to $A G$.

(Prop. IV.)

Again, produce the edges $D^{\prime} A^{\prime}, C^{\prime} B^{\prime}, G^{\prime} F^{\prime}, H^{\prime} E^{\prime}$, of the parallelepiped $A^{\prime} G^{\prime}$, and cut them perpendicularly at $D^{\prime \prime}, C^{\prime \prime}$, $G^{\prime \prime}, H^{\prime \prime}$, and $A^{\prime \prime}, B^{\prime \prime}, F^{\prime \prime}, E^{\prime \prime}$, by two parallel planes whose distance apart $B^{\prime \prime} C^{\prime \prime}$ is equal to $B^{\prime} C^{\prime}$.

Then $A^{\prime \prime} G^{\prime \prime}$ is a rectangular parallelepiped [why ?] equal in volume to $A^{\prime} G^{\prime}$ (Prop. IV), and hence equal to $A G$.

Now the volume of $A^{\prime \prime} G^{\prime \prime}$ is equal to the product of the altitude $A^{\prime \prime} E^{\prime \prime}$ and the area of the base $A^{\prime \prime} C^{\prime \prime}$.

(Art. 504.)

But since all three parallelepipeds lie between the same two parallel planes, the altitude $K E$ equals the altitude $A^{\prime \prime} E^{\prime \prime}$. 
Also the parallelograms $A C, A^{\prime} C^{\prime}$, and $A^{\prime \prime} C^{\prime \prime}$ are equal in area.

Therefore, volume of $A G=$ volume of $A^{\prime \prime} G^{\prime \prime}$

(Art. 294)

$$
\begin{aligned}
& =\text { area of } A^{\prime \prime} C^{\prime \prime} \times A^{\prime \prime} E^{\prime \prime} \\
& =\text { area of } A C \times K E .
\end{aligned}
$$

\section{Proposition XI}

506. The volume of a triangular prism is equal to the product of its altitude and the area of its base.

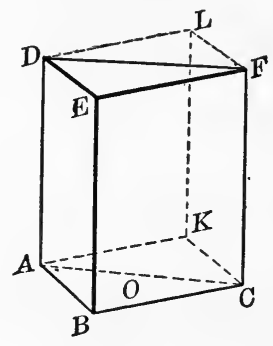

Let $A B C-D E F$ be any triangular prism whose altitude is $h$.

It is required to prove that the volume of this prism is equal to $h$ times the area of the base $A B C$.

Proof. Complete the parallelogram $A B C K$. Through $K$ draw a line parallel to a lateral edge $A D$, meeting the plane $D E F$ at $L$, and complete the parallelepiped $B L$ having the same altitude as the prism.

The prism $A B C-D E F$ is one-half of the parallelepiped $B L$.

(Prop. VI.)

Now the volume of $B L$ is the product of its altitude $h$ and the area of the base $A B C K$.

(Prop. X.)

Therefore the volume of the prism $A B C-D E F$ is equal to $h$ times the area of $A B C$, which is half of the base $A B C K$. 
507. Corollary I. The volume of any prism is equal to the product of its altitude and the area of its base.

Any prism can be divided into triangular prisms by passing planes through one lateral edge and all the nonadjacent lateral edges.

508. Corollary II. The volumes of any two prisms are equal if they have equal altitudes and

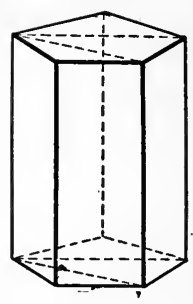
bases of equal areas.

\section{EXERCISES}

1. The square on the diagonal of a rectangular parallelepiped is equal to the sum of the squares on the three edges meeting in one extremity of the diagonal.

2. The square on the diagonal of a cube is three times the square on one of its edges.

3. Show that all the diagonals of a rectangular parallelepiped are equal.

4. The sum of the squares on the four diagonals of a parallelepiped is equal to the sum of the squares on the twelve edges.

5. Every section of a prism made by a plane parallel to an edge is a parallelogram.

6. Find the volume and the length of the diagonal of a cube whose edge is 3 feet.

7. Show that all the diagonals of any parallelepiped pass through one point, and are bisected at that point.

8. The edge of a cube is 4 feet. Find the edge of a cube having twice the volume.

9. The dimensions of a rectangular parallelepiped are 3,5 , and 7 decimeters. Find its volume and the length of its diagonals.

10. A box, covered top and bottom, which is made of boards 2 inches thick, has outside dimensions of 18,24 , and 30 inches. Find its entire contents.

11. Find the volume of a right prism whose base is a regular hexagon of 6 inches side, and whose altitude is 15 inches.

12. The diagonal of a cube is 27 inches, find its volume. 


\section{Section II}

\section{PYRAMIDS}

509. A pyramid is a polyhedron one of whose faces is a poly. gon, and the remaining faces are triangles having a common vertex.

The polygonal face is called the base of the pyramid, the triangular faces are the lateral faces, and the common vertex of the lateral faces is the vertex of the pyramid.

The sum of the areas of the lateral faces is called

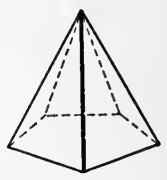
the lateral area of the pyramid.

The perpendicular distance from the vertex to the base is the altitude of the pyramid.

A triangular pyramid is one whose base is a triangle; a quadrangular pyramid, one whose base is a quadrilateral, etc.

A triangular pyramid is a tetrahedron since it has four faces.

All the faces of a tetrahedron are triangles. Any face may be taken as the base, and the opposite vertex as the vertex.

How many vertices has a tetrahedron? how many edges? how many pairs of non-intersecting edges? These are called the pairs of opposite edges.

510. A regular pyramid is one whose base is a regular poly. gon and whose vertex lies on the perpendicular to the base drawn from its centre.

The slant height of a regular pyramid is the altitude of any one of its lateral faces; i.e. it is the perpendicular distance of the vertex from any side of the base.

511. A truncated pyramid is the figure formed by the base and any section of a pyramid, and the por. tions of the lateral faces intercepted between them.

If the upper section is parallel to the base, the figure is called a frustum of a pyramid.

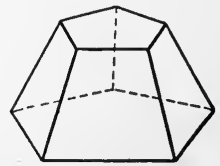


The altitude of a frustum of a pyramid is the perpendicular distance between its bases.

The slant height of the frustum of a regular pyramid is the perpendicular distance between the parallel edges of a lateral face.

\section{Proposition XII}

512. The lateral area of a regular pyramid is equal to one-half the product of the perimeter of the base and the slant height.

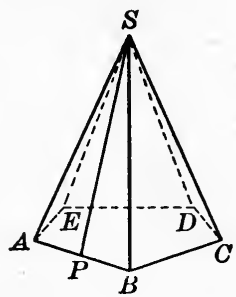

Let $S-A B C D E$ be any regular pyramid whose slant height is $S P$.

It is required to prove that its lateral area is equal to onehalf the product of the perimeter of the polygon $A B C D E$ and the slant height $S P$.

Proof. 1. What is the area of $\triangle S A B$ ?

2. $\triangle \mathrm{s} S A B, S B C$, etc., have equal altitudes.

(Art. 306.)

3. What is the area of all the triangles?

4. What is the lateral area?

\section{EXERCISES}

1. Prove that the lateral edges of a regular pyramid are all equal, and hence that the lateral faces are equal isosceles triangles.

2. Show that the lateral faces of the frustum of a pyramid are all trapezoids, and of a regular pyramid are all equal trapezoids.

3. Show that the lateral area of the frustum of a regular pyramid is equal to half the sum of the perimeters of its bases multiplied by the slant height. 


\section{Proposition XIII}

513. If a pyramid is cut by a plane parcllel to its base, the edges and the altitude are divided in the same ratio, the section made by the plane is similar to the base, and the area of the section is to the area of the base as the squares of their distances from the vertex.

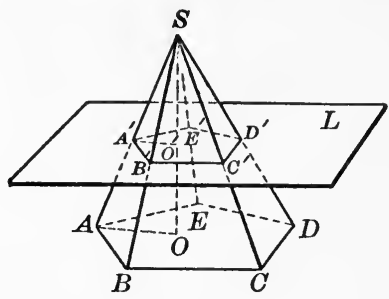

Let $S-A B C D E$ be any pyramid whose altitude is $S O$, and let it be cut by a plane $L$ parallel to the base, in the polygon $A^{\prime} B^{\prime} C^{\prime} D^{\prime} E^{\prime}$ whose distance from $S$ is $S O^{\prime}$.

It is required to prove

1. That $\frac{S A^{\prime}}{S A}=\frac{S B^{\prime}}{S B}=\frac{S C^{\prime}}{S C}=$ etc. $=\frac{S O^{\prime}}{S O}$.

2. That $A^{\prime} B^{\prime} C^{\prime} D^{\prime} E^{\prime}$ is similar to $A B C D E$.

3. That area of $A^{\prime} B^{\prime} C^{\prime} D^{\prime} E^{\prime}$ : area of $A B C D E={\overline{S O^{\prime}}}^{2}: \overline{S O}^{2}$.

Proof. First. In $\triangle S A B, A^{\prime} B^{\prime}$ is parallel to $A B$. (Art. 419.)

Therefore

$$
\frac{S A^{\prime}}{S A}=\frac{S B^{\prime}}{S B} \text {. }
$$

Similarly,

$$
\frac{S B^{\prime}}{S B}=\frac{S C^{\prime}}{S C^{\prime}}, \cdots
$$

Also, since $O^{\prime}$ must lie upon $S O, A^{\prime} O^{\prime}$ is parallel to $A O$.

Therefore

$$
\begin{aligned}
\frac{S A^{\prime}}{S A} & =\frac{S O^{\prime}}{S O} . \\
\frac{S A^{\prime}}{S A}=\frac{S B^{\prime}}{S B} & =\frac{S C^{\prime}}{S C}=\cdots=\frac{S O^{\prime}}{S O} .
\end{aligned}
$$

Whence 
Second. Since $A^{\prime} B^{\prime}$ is parallel to $A B$, and $B^{\prime} C^{\prime}$ is parallel to $B C$,

$$
\angle A^{\prime} B^{\prime} C^{\prime}=\angle A B C \text {. }
$$

Similarly,

$$
\angle B^{\prime} C^{\prime} D^{\prime}=\angle B C D, \ldots
$$

Hence the polygons $A^{\prime} B^{\prime} C^{\prime} D^{\prime} E^{\prime}$ and $A B C D E$ are mutually equiangular.

Also,

$$
\frac{A^{\prime} B^{\prime}}{A B}=\frac{S B^{\prime}}{S B} .
$$

Similarly,

$$
\frac{S B^{\prime}}{S B}=\frac{B^{\prime} C^{\prime}}{B C}, \cdots
$$

Therefore

$$
\frac{A^{\prime} B^{\prime}}{A B}=\frac{B^{\prime} C^{\prime}}{B C}=\frac{C^{\prime} D^{\prime}}{C D}=\text { etc. }
$$

Hence, in the polygons $A^{\prime} B^{\prime} C^{\prime} D^{\prime} E^{\prime}$ and $A B C D E$, pairs of homologous sides are in the same ratio.

Therefore the polygons $A^{\prime} B^{\prime} C^{\prime} D^{\prime} E^{\prime}$ and $A B C D E$ are similar.

Third. Area of $A^{\prime} B^{\prime} C^{\prime} D^{\prime} E^{\prime}$ : area of $A B C D E$

$$
\begin{aligned}
& ={\overline{A^{\prime} B^{\prime}}}^{2}: \overline{A B}^{2} \\
& ={\overline{S A^{\prime}}}^{2}: \overline{S A}^{2} \\
& ={\overline{S O^{\prime}}}^{2}: \overline{S O}^{2} .
\end{aligned}
$$

514. Corollary I. Sections of the same pyramid made by parallel planes are similar.

515. Corollary II. The areas of parallel sections of a pyramid are in the same ratio as the squares of their distances from the vertex.

516. Corollary III. If two pyramids have equal altitudes and bases of equal areas, the areas of sections made by planes equidistant from their vertices are equal. 


\section{Proposition XIV}

517. If two triangular pyramids have equal altitudes and equal bases, they have equal volumes.
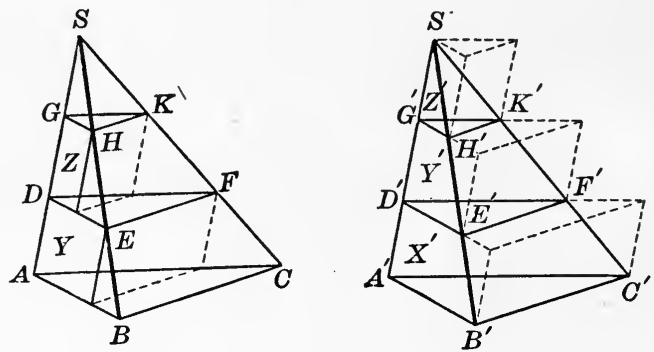

Let $S-A B C$ and $S^{\prime}-A^{\prime} B^{\prime} C^{\prime}$ be two triangular pyramids having their bases $A B C$ and $A^{\prime} B^{\prime} C^{\prime}$ equal in area, and their altitudes $S O$ and $S^{\prime} O^{\prime}$ also equal.

It is required to prove that the volume of $S-A B C$ is equal to the volume of $S^{\prime}-A^{\prime} B^{\prime} C^{\prime \prime}$.

Proof. Divide the altitudes of the pyramids $S$ and $S^{\prime}$ into any number of equal parts, each into the same number, and through the points of division pass planes parallel to the bases.

Let the sections made by these planes be $D E F, G H K$, etc., in the one pyramid, and $D^{\prime} E^{\prime} F^{\prime}, G^{\prime} H^{\prime} K^{\prime}$, etc., in the other.

Then

the area of $D E F=$ the area of $\bar{D}^{\prime} E^{\prime} F^{\prime}$, (Art. 516.) area of $G H K=$ area of $G^{\prime} H^{\prime} K^{\prime}$, etc.

With the sections DEF, GHK, etc., as upper bases, construct prisms $Y, Z$, etc., having their edges equal and parallel to $D A, G D$, etc.

Also with $A^{\prime} B^{\prime} C^{\prime}, D^{\prime} E^{\prime} F^{\prime}, G^{\prime} H^{\prime} K^{\prime}$, etc., as lower bases, construct prisms $X^{\prime}, Y^{\prime}, Z^{\prime}$, etc., having their edges equal and parallel to $D^{\prime} A^{\prime}, G^{\prime} D^{\prime}, S^{\prime} G^{\prime}$, etc. 
These prisms are all of the same altitude, and in volume $Y=Y^{\prime}, Z=Z^{\prime}$, etc.

Therefore the sum of all the prisms in the figure $S^{\prime}$ is greater than the sum of all the prisms in the figure $S$ by the lowest prism $X^{\prime}$.

Denote the sum of the volumes of the prisms in the figure $S$ by $P$, in the figure $S^{\prime}$ by $P^{\prime}$, and the volume of $X^{\prime}$ by $E$.

Then $P=P^{\prime}-E$.

Now, if the number of parts into which the altitudes are divided is indefinitely increased, and the altitudes of the prisms consequently indefinitely diminished, $P$ will approach the volume of the pyramid $S-A B C$ as its limit, $P^{\prime}$ will approach the volume of the pyramid $S^{\prime}-A^{\prime} B^{\prime} C^{\prime}$ as its limit, while $E$ will become indefinitely small, and is ultimately less than any assignable quantity.

Therefore the difference between the volumes of the pyramids $S-A B C$ and $S^{\prime}-A^{\prime} B^{\prime} C^{\prime}$ is less than any assignable quantity. That is, the volumes of the two pyramids are equal.

518. Note. The test for geometrical equality is superposition.

That is to say, two geometrical magnitudes are equal when they can be made to occupy the same position.

The test for numerical equality is given by the following definition :

Two numerical magnitudes are equal when their difference is less than any assignable quantity.

519. Corollary I. Triangular pyramids upon the same base and having their vertices in a plane parallel to the base, are equal - in volume.

The vertex of a tetrahedron may be moved anywhere in a plane parallel to the base without altering the volume.

520. Corollary II. Triangular pyramids having equal bases in the same plane and their vertices at the same point, are equal in volume.

If the base of a pyramid is a parallelogram, the plane through the vertex and two opposite vertices of the base divides the pyramid into two triangular pyramids having equal volumes. 


\section{Proposition XV}

521. The volume of a triangular pyramid is onethird the volume of a triangular prism of the same base and altitude.

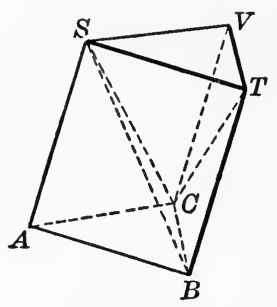

Let $S-A B C$ be any triangular pyramid and $A B C-S T V$ be a triangular prism having the same base and altitude.

It is required to prove that the volume of $S-A B C$ is equal to one-third the volume of $A B C-S T V$.

Proof. In the face $B C V T$ of the prism draw the diagonal $C T$, and through the points $S, C, T$, pass a plane.

The given prism is thus composed of the three triangular pyramids $S-A B C, S-B C T$, and $S-V C T$.

Now the volumes of the two pyramids $S-B C T$ and $S-V C T$ are equal. Why?

The pyramid $S-V C T$ may also be read $C-S V T$.

And the volume of $C-S V T$ is equal to the volume of $S-A B C$. Why?

Therefore the prism $A B C-S T V$ is composed of three triangular pyramids, each of which is equal in volume to $S-A B C$. Therefore the volume of $S-A B C$ is equal to one-third of the volume of the prism $A B C-S T V$.

522. Conollary I. The volume of a triangular pyramid is equal to one-third the product of its altitude and the area of its base. (Art. 506.) 
523. Corollary II. The volume of any pyramid is equal to one-third the product of its altitude and the area of its base.

The pyramid can be divided into triangular pyramids by passing planes through the vertex.

524. Corollary III. The volumes of any two pyramids are in the same ratio as the products of their altitudes by the areas of their bases.

If $V$ and $V^{\prime}$ are the volumes of two pyramids, $A$ and $A^{\prime}$ the areas of their bases, $h$ and $h^{\prime}$ their altitudes,

then

$$
V=\frac{1}{3} h A, \quad V^{\prime}=\frac{1}{3} h^{\prime} A^{\prime} .
$$

Whence

$$
\frac{V}{V^{\prime}}=\frac{\frac{1}{3} h A}{\frac{1}{3} h^{\prime} A^{\prime}}=\frac{h A}{h^{\prime} A^{\prime}} .
$$

525. Corolla Ry IV. The volumes of two pyramids having equal altitudes are in the same ratio as the areas of their bases, or having equal bases are in the same ratio as their altitudes.

\section{EXERCISES}

1. A pyramid -is cut by a plane midway between the vertex and the base. Find the ratio of the area of the section to the area of the base.

2. In the figure of Proposition XIV, assuming the altitude of the pyramid $S$ to have been divided into three equal parts, show that the volume of the prism $Y$ is four times the volume of the prisn $Z$.

3. The section of a pyramid made by a plane parallel to the base is equal in area to four-ninths of the area of the base. In what ratio does the plane of section divide the lateral area?

4. A regular pyramid and a regular prism have equal hexagonal bases, and altitudes equal to three times the radius of the circles circumscribing the bases. What is the ratio of their lateral areas ?

5. Find the lateral area and the volume of a regular octagonal pyramid whose slant height is 5 metres and a side of whose base is 2 metres.

6. The total surface of a regular pyramid, whose base is a square of 10 feet side, is 360 square feet. Find its altitude. 


\section{Proposition XVI}

526. The volumes of two tetrahedrons having a trihedral angle of the one equal to a trihedral angle of the other, are in the same ratio as the products of the edges which meet in the vertices of these angles.

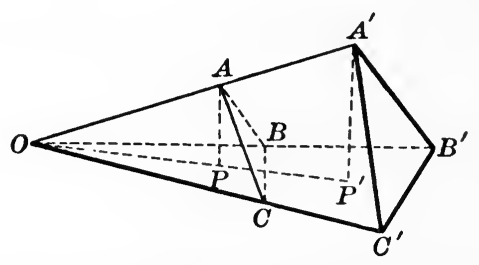

Let $O-A B C$ and $O-A^{\prime} B^{\prime} C^{\prime \prime}$ be two tetrahedrons having the trihedral angles at $O$ equal, and let their volumes be $V$ and $V^{\prime}$.

It is required to prove that

$$
V: V^{\prime}=A O \times B O \times C O: A^{\prime} O \times B^{\prime} O \times C^{\prime \prime} O .
$$

Proof. Place the tetrahedrons so that their equal trihedral angles coincide.

From $A$ and $A^{\prime}$ draw perpendiculars $A P$ and $A^{\prime} P^{\prime}$ to the plane $O B C$. These will lie in a plane with $O A A^{\prime}$ and will meet $O B C$ in points of a straight line $O P P^{\prime}$.

(Art. 405.)

Then

$$
\frac{A P}{A^{\prime} P^{\prime}}=\frac{A O}{A^{\prime} O} . \text { Why? }
$$

Also

$$
\begin{gathered}
\text { volume of } O-A B C \\
=\frac{A P \times \text { area of } O B C}{A^{\prime} P^{\prime} \times \text { area of } O B^{\prime} C^{\prime \prime}} \\
=\frac{A O}{A^{\prime} O} \cdot \frac{\text { area of } O B C}{\text { area of } O B^{\prime} C^{\prime \prime}}
\end{gathered}
$$

Now

$$
\frac{\text { area of } O B C}{\text { area of } O B^{\prime} C^{\prime}}=\frac{B O \times C O}{B^{\prime} O \times C^{\prime} O} \text {. }
$$

Therefore $\frac{\text { volume of } O-A B C}{\text { volume of } O-A^{\prime} B^{\prime} C^{\prime}}=\frac{A O \times B O \times C O}{A^{\prime} O \times B^{\prime} O \times C^{\prime} O}$. 


\section{Proposition XVII}

527. The volume of a truncated triangular prism is equal to the sum of the volumes of three pyramids whose common base is one base of the prism and whose vertices are the three vertices of the other base.

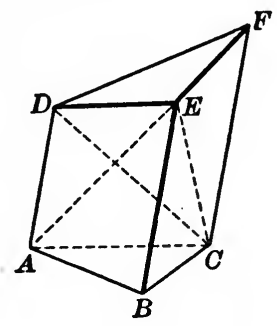

Let $A B C-D E F$ be any truncated triangular prism.

It is required to prove that the volume of this figure is equal to the sum of the volumes of three pyramids whose common base is $A B C$, and whose vertices are $D, E, F$, respectively.

Proof. Through the points $E, A, C$, and $E, D, C$, pass planes, thus dividing the given figure into the three pyramids $E-A B C, E-A D C$, and $E-D F C$.

The first of these, viz. $E-A B C$, has the base $A B C$ and vertex $E$.

The second, $E-A D C$ may be read $C-A E D$.

Now the base $A E D$ is equal in area to the base $A B D$. (Art. 296.)

Therefore the volume of $C-A E D$ is equal to the volume of $C-A B D$ (Prop. XIV), which is the same as $D-A B C$.

The third, $E-D F C$, is equal in volume to $E-A F C$, which is the same as $A-E F C$. And this is equal in volume to $A-B F C$, which is the same as $F-A B C$.

Therefore the prism $A B C-D E F$ is equal in volume to the pyramids $E-A B C+D-A B C+F-A B C$. 
528. Corollary I. The volume of a truncated right triangular prism is equal to the product of the area of that face to which the edges are perpendicular and one-third the sum of the lateral edges.

For the lateral edges are the altitudes of the three pyramids to the sum of which the volume of the prism is equal.

529. Corollary II. The volume of any truncated triangular prism is equal to the product of the area of a right section and one-third the sum of the lateral edges.

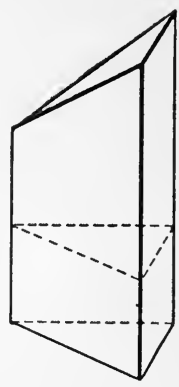

On either side of the right section lies a truncated right prism.

\section{EXERCISES}

1. Any two vertices of a tetrahedron may move along straight lines drawn through them parallel to the edge through the other two, without altering the volume of the tetrahedron.

The proof of this theorem is contained in Proposition XVII, where it was shown that the volume of the tetrahedron $E-D F C$ is unaltered when $E$ moves to $B$, and $D$ to $A$.

2. If $A$ is the area of the lower base of a frustum of a pyramid, $a$ the area of the upper base, and $h$ the altitude, then the volume of the frustum $=\frac{h}{3}(A+a+\sqrt{A a})$.

Let $l$ be the altitude, above the base $a$, of the pyramid of which the figure considered is a frustum.

Then

$$
A: a=(h+l)^{2}: l^{2} \text {, }
$$

or

$$
\sqrt{A}: \sqrt{a}=h+l: l \text {, }
$$

and

$$
\sqrt{A}-\sqrt{a}: \sqrt{a}=h: l \text {; }
$$

whence

$$
\begin{aligned}
& l=\frac{h \sqrt{a}}{\sqrt{A}-\sqrt{a}} \text { and } h+l=\frac{\sqrt{A}}{\sqrt{a}} \cdot l=\frac{h \sqrt{A}}{\sqrt{A}-\sqrt{a}} \cdot \\
& \text { Volume }=\frac{1}{3} A(h+l)-\frac{1}{3} a l=\frac{1}{3}\left[\frac{A h \sqrt{A}}{\sqrt{A}-\sqrt{a}}-\frac{a h \sqrt{a}}{\sqrt{A}-\sqrt{a}}\right] \\
& \quad=\frac{h}{3}\left[\frac{A \sqrt{A}-a \sqrt{a}}{\sqrt{A}-\sqrt{a}}\right]=\frac{h}{3}(A+a+\sqrt{A a}) .
\end{aligned}
$$

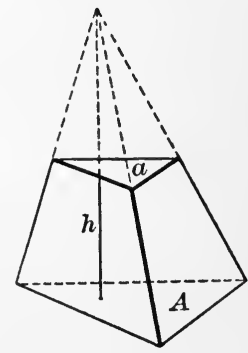




\section{SECTION III}

\section{SIMILAR POLYHEDRONS}

530. Definition. Two polyhedrons are said to be similar when they have the same number of faces similar each to each and similarly placed, and have their corresponding polyhedral angles identically equal.

Similar polyhedrons not only have the same number of faces, but also the same number of vertices and the same number of edges. They have the same number of faces meeting at corresponding vertices, and consequently the same number of edges meeting at corresponding vertices.

If two prisms are similar, their bases must be similar polygons, and their homologous lateral faces similar parallelograms; while if two pyramids are similar, their bases are similar polygons, and their homologous lateral faces are similar triangles.

531. Since corresponding faces in two similar polyhedrons are similar polygons, and homologous sides of two similar polygons are proportional,

Therefore, any two edges or diagonals of a polyhedron are in the same ratio as the homologous edges or diagonals in a similar polyhedron.

Show that this is true when the edges do not lie in the same face of the polyhedron.

532. The areas of two similar polygons are in the same ratio as the squares of any two homologous sides. (Art. 315.)

Therefore, the surfaces of two similar polyhedrons (i.e. the sums of the areas of all the faces) are in the same ratio as the squares of any two homologous edges or homologous diagonals.

\section{EXERCISES}

1. A plane parallel to the base cuts from the top of a pyramid a figure similar to the given pyramid. Can the same be said of a prism? 


\section{Proposition XVIII}

533. Two tetrahedrons are similar if the three faces meeting in a vertex of the one are similar and similarly situated to the three faces meeting in a vertex of the other.
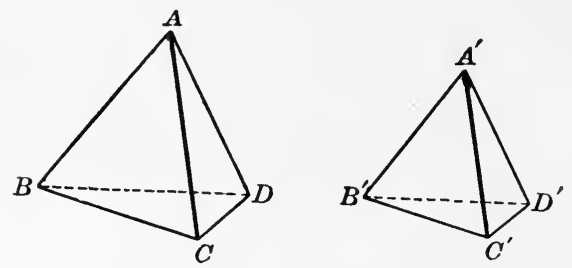

If the tetrahedrons are $A-B C D$ and $A^{\prime}-B^{\prime} C^{\prime} D^{\prime}$, and the faces meeting at the vertex $A$ are similar and similarly situated to the faces meeting at the vertex $A^{\prime}$, the face opposite $A$ is similar and similarly situated to the face opposite $A^{\prime}$, since the three sides of one of these faces are proportional to the three sides of the other.

(Art. 253.)

Also the trihedral angle $A$ is equal to the trihedral angle $A^{\prime}$, since the three face angles of the one are equal, respectively, and are similarly placed to the three face angles of the other.

(Art. 461.)

For the same reason the trihedral angles $B, C, D$, are equal, respectively, to the trihedral angles $B^{\prime}, C^{\prime}, D^{\prime}$.

Therefore the tetrahedrons are similar.

534. Theorem. Any two similar polyhedrons can be divided into the same number of tetrahedrons, similar two and two.

For, if $A B, A C, A D$, and $A^{\prime} B^{\prime}, A^{\prime} C^{\prime}, A^{\prime} D^{\prime}$ are consecutive homologous edges of the two polyhedrons, meeting in the homologous vertices $A$ and $A^{\prime}$ the three pairs of planes determined by these edges, together with the planes $B C D$ and $B^{\prime} C^{\prime} D^{\prime}$, form the faces of two tetrahedrons which are similar by the above proposition, and are similarly placed. This process of cutting off similar tetrahedrons can be continued till only two similar tetrahedrons remain. 


\section{Proposition XIX}

535. The volumes of two similar tetrahedrons are in the same ratio as the cubes of their homologous edges.

Let $A-B C D$ and $A^{\prime}-B^{\prime} C^{\prime} D^{\prime}$ be two similar tetrahedrons whose volumes are denoted by $V$ and $V^{\prime}$.

It is required to prove that $V: V^{\prime}=\overline{A B}^{3}:{\overline{A^{\prime} B^{\prime}}}^{3}$.

Proof. If $A$ and $A^{\prime}$ are two homologous vertices, the trihedral angles $A$ and $A^{\prime}$ must be equal by definition.

Therefore

$$
\begin{aligned}
\frac{V}{V^{\prime}} & =\frac{A B \times A C \times A D}{A^{\prime} B^{\prime} \times A^{\prime} C^{\prime} \times A^{\prime} D^{\prime}} \\
& =\frac{A B}{A^{\prime} B^{\prime}} \times \frac{A C}{A^{\prime} C^{\prime}} \times \frac{A D}{A^{\prime} D^{\prime}} .
\end{aligned}
$$

But

$$
\frac{A B}{A^{\prime} B^{\prime}}=\frac{A C}{A^{\prime} C^{\prime}}=\frac{A D}{A^{\prime} D^{\prime}} \text {, by hypothesis. }
$$

Therefore

$$
\frac{V}{V^{\prime}}=\frac{A B}{A^{\prime} B^{\prime}} \times \frac{A B}{A^{\prime} B^{\prime}} \times \frac{A B}{A^{\prime} B^{\prime}}=\frac{\overline{A B}^{3}}{{\overline{A^{\prime} B^{\prime}}}^{3}} .
$$

536. Corollary. The volumes of any two similar polyhedrons are in the same ratio as the cubes of two homologous edges.

For the polyhedrons can be divided into the same number of similar tetrahedrons, and any two of these which are similarly placed are in the same ratio as the cubes of homologous edges. Hence the sums of all the tetrahedrons are in the same ratio as the cubes of homologous edges. (Art. 240.)

\section{EXERCISES}

1. Any two cubes are similar polyhedrons, and hence their volumes are in the same ratio as the cubes of homologous, or any, edges.

2. A plane is passed through the mid-points of three edges of a tetrahedron ; compare the volume of the original tetrahedron with the volumes of the parts into which it is divided by the plane. 


\section{SECTION IV}

\section{REGULAR POLYHEDRONS}

537. Definition. A regular polyhedron is one whose faces are equal regular polygons and whose polyhedral angles are all equal.

Since the polyhedral angles are all equal, the same number of faces and edges must meet at each vertex; and since the faces are all equal, the same number of vertices and edges must lie on each face.

All the edges of a regular polyhedron are equal.

The faces of a convex regular polyhedron are all convex polygons, and the polyhedral angles are all convex.

538. At each vertex there must meet at least three faces, and in each face there must lie at least three vertices.

Moreover, the sum of the face angles at any vertex of a convex regular polyhedron is less than four right angles, or 360 degrees.

(Art. 460.)

From these two properties we are able to show that the greatest possible number of convex regular polyhedrons is five.

The faces of a regular convex polyhedron must be regular convex polygons, equilateral triangles, squares, etc.

1. Since the interior angle of an equilateral triangle is 60 degrees, if the faces of a regular convex polyhedron are equilateral triangles, at each vertex there may meet three, four, or five faces; but not six.

2. Since the interior angle of a square is 90 degrees, if the faces of a regular convex polyhedron are squares, at each vertex there may meet three faces, but not four.

3. Since the interior angle of a regular pentagon is $\mathbf{1 0 8}$ degrees, if the faces of a regular convex polyhedron are pentagons, at each vertex there may meet three faces, but not four.

4. The interior angle of a regular hexagon is 120 degrees; hence it is impossible for three or more regular hexagons to 
meet at a vertex so as to form a convex polyhedral angle. Similarly no regular polygon of more than six sides can be a face of a regular convex polyhedron.

Therefore the only polygons which can enter into the construction of regular convex polyhedrons are equilateral triangles, squares, and regular pentagons and the ways in which these can be combined are :

(1) triangular faces meeting three at a vertex.

(2) triangular faces meeting four at a vertex.

(3) triangular faces meeting five at a vertex.

(4) square faces meeting three at a vertex.

(5) pentagonal faces meeting three at a vertex.

What is here shown is that no other regular convex polyhedrons than the five answering the above conditions can exist; that these five varieties do exist can most easily be demonstrated by actually making models of them.

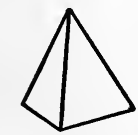

Tetrahedron

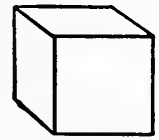

Hexahedron

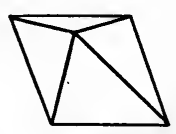

Octahedron

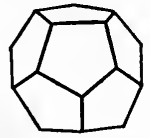

Dodecahedron

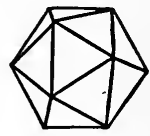

Icosahedron

539. The five regular polyhedrons are:

1. The regular tetrahedron, having four triangular faces meeting three in a vertex.

It has four vertices and six edges.

2 . The regular octahedron, having eight triangular faces meeting four in a vertex.

It has six vertices and twelve edges.

3. The regular icosahedron, having twenty triangular faces meeting five in a vertex.

It has twelve vertices and thirty edges.

4. The regular hexahedron or cube, having six square faces meeting three in a vertex.

It has eight vertices and twelve edges. 
5. The regular dodecahedron, having twelve pentagonal faces meeting three in a vertex.

It has twenty vertices and thirty edges.

540. Models of the five regular convex polyhedrons can easily be constructed by cutting pieces of cardboard in the shape of the diagrams below, and folding them along the dotted lines till the edges come together. The edges should then be glued or pasted over with strips of cloth or paper. To make the folding easy it would be well to cut the cardboard halfway through along the dotted lines.
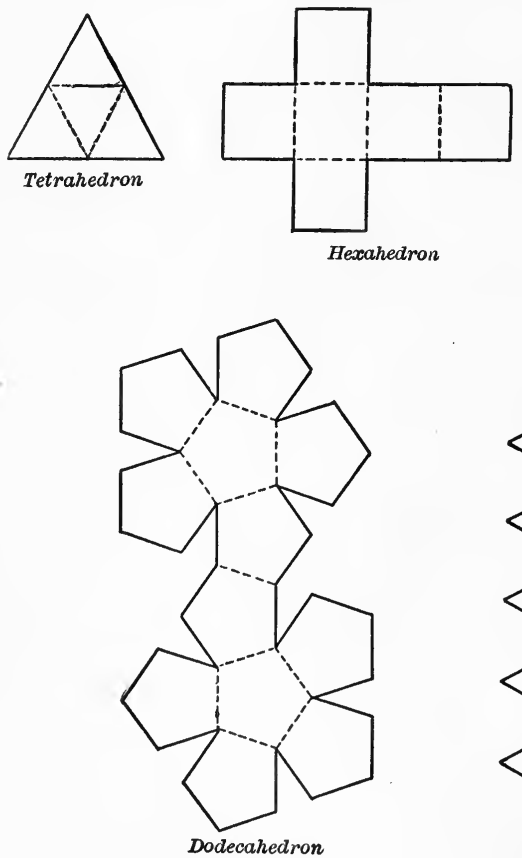
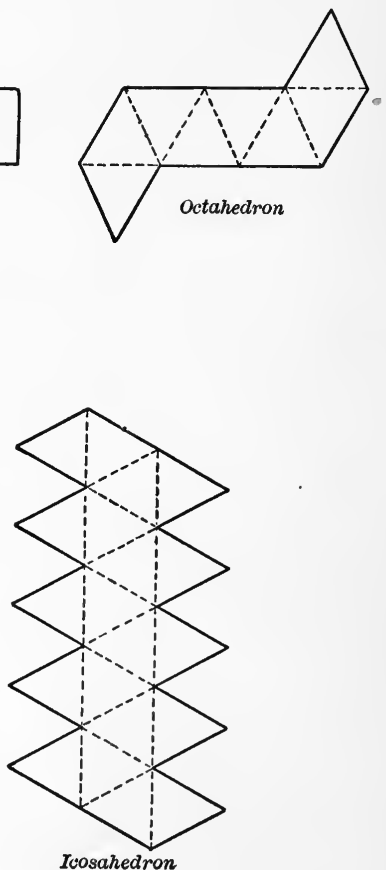


\section{Section V \\ POLYHEDRONS IN GENERAL \\ Proposition XX}

541. In any polyhedron the number of edges increased by two is equal to the number of faces together with the number of vertices. [Euler's Theorem.]

Let $E$ be the number of edges in any given polyhedron, $F$ the number of faces, and $V$ the number of vertices.

It is required to prove that $E+2=V+F$.

Proof. Imagine the polyhedron to be built up by taking one face and to it attaching another, and another, and so on, till the whole figure is completed.

Let $f$ be the number of faces of the incomplete figure at any stage, $e$ the number of edges, and $v$ the number of vertices.

In any face taken alone, the number of vertices equals the number of edges.

So that when $f=1, \quad e=v$.

Now to the first face add a second. One edge of the second is made to coincide with an edge of the first, while two vertices of the second coincide with two vertices of the first.

That is, in the new face by itself the number of edges equals the number of vertices, but when this face is attached to the one already taken, two vertices are lost, while only one edge is lost.

Therefore, when $f=2, \quad e=v+1$.

To these attach a third face, and again the number of vertices lost will exceed the number of edges lost by one.

Therefore, when $f=3, \quad e=v+2$.

Continue this process till all the faces are added but the last one. 
Then, when $f=F-1, e=v+(F-2)$, since the number of vertices lost for each of the $F-2$ faces added has exceeded the number of edges lost by one.

When the last face is added, all of its edges and vertices are made to coincide with edges and vertices already counted. So that adding the last face merely completes the figure without changing the relation between the numbers of edges and vertices.

Therefore, for the complete figure,

or

$$
\begin{gathered}
E=V+(F-2), \\
E+2=V+F .
\end{gathered}
$$

\section{Proposition XXI}

542. The sum of the face angles of any polyhedron, together with eight right angles, is equal to four times as many right angles as the polyhedron has vertices.

Let $F$ denote the number of faces, $E$ the number of edges, $V$ the number of vertices, and $S$ the sum of the face angles of any polyhedron.

It is required to prove that $S+8$ right angles $=4 \mathrm{~V}$ right angles.

Proof. In any face, the sum of the face angles +4 right angles $=$ twice as many right angles as the face has edges.

(Art. 116.)

Therefore, adding for all the faces, remembering that each edge is counted twice,

$$
S+4 F \text { right angles }=2 E \times 2 \text { right angles, }
$$

or $\quad S=4\left(E-F^{\prime}\right)$ right angles

$$
=4(V-2) \text { right angles, }
$$

i.e. $\quad S+8$ right angles $=4 V$ right angles. 


\section{MISCELLANEOUS EXERCISES}

1. There can be no polyhedron with less than four faces, or less than six edges.

2. No polyhedron can be constructed having seven edges.

3. The sum of the areas of any three faces of a tetrahedron is greater than the area of the fourth face.

4. If a tetrahedron is cut by a plane parallel to a pair of opposite edges, the section will be a parallelogram.

5. The straight lines which join the mid-points of opposite edges of a tetrahedron are concurrent.

6. If a perpendicular is drawn from a vertex of a regular tetrahedron to its opposite face, show that the foot of the perpendicular will intersect each median of that face, dividing it in the ratio $2: 1$.

7. Show that the perpendicular from the vertex of a regular tetrahedron upon the opposite face is three times that drawn from its foot to any of the other faces.

8. Prove that the lateral area of any pyramid is greater than the area of the base.

Suggestion. Project the vertex on the base and join its projection to the vertices of the base.

9. If planes are passed through three concurrent edges of a tetrahedron and the mid-points of their opposite edges, they will intersect in one straight line.

10. The volume of a triangular prism is equal to one-half the product of the area of one lateral face and the perpendicular distance of it from the opposite edge.

11. The plane which bisects a dihedral angle of a tetrahedron cuts the opposite edge into segments proportional to the areas of 'the adjacent faces.

12. The altitude of a regular tetrahedron is equal to the sum of the four perpendiculars drawn from any point within it to the four faces.

13. The area of any lateral face of a prism is less than the sum of the areas of the other lateral faces.

14. Show that the six planes bisecting the dihedral angles of any tetrahedron meet in one point.

- Suggestion. Compare Ex. 1, p. 303. 
15. The six planes each passing through one edge of a tetrahedron and the mid-point of the opposite edge, meet in one point.

16. Given the number and kind of faces in each of the regular polyhedrons, and the number of faces meeting at a vertex, compute the number of vertices and edges.

For example, in the regular dodecahedron there are twelve pentagonal faces meeting three in a vertex.

12 faces, 5 vertices in each face gives 60 face angles.

3 face angles at each vertex, gives $\frac{60}{3}=20$ vertices.

Also 12 faces, 5 edges in each face gives 60 edges, but this counts each edge twice, since each edge lies in two faces.

Therefore the actual number of edges is $\frac{60}{2}=30$ edges.

17. Verify Euler's formula, $E+2=V+F$, for the case of each of the regular convex polyhedrons.

18. Verify Euler's formula for the case of a hexagonal pyramid and a hexagonal prism.

19. The total number of plane angles in the faces of any polyhedron is even, and equal to twice the number of edges.

20. The homologous edges of two similar tetrahedrons are in the ratio $5: 6$. Find the ratio of their total areas and their volumes.

21. One edge of a tetrahedron is 6 inches. Find the length of the homologous edge of a similar tetrahedron having (1) twice the volume, (2) twice the total area.

22. Two parallelepipeds are similar if the three faces meeting at any vertex are similar and similarly placed.

23. If four straight lines, of which no three lie in the same plane, intersect in one point, and are cut by two parallel planes on opposite sides of their common point, show that the eight points of intersection are the vertices of a parallelepiped.

24. The base of a pyramid is 12 square feet and its altitude is 6 feet. Find the area of a section parallel to the base and 2 feet from it.

25. The bases of a frustum of a pyramid are 8 square feet and $4 \frac{1}{2}$ square feet, respectively, and its altitude is 5 feet. Find its volume.

26. A cistern is 8 feet square at the top, 6 feet square at the bottom, and 8 feet deep. How many gallons of water will it hold, assuming $7 \frac{1}{2}$ gallons to the cubic foot? 


\section{SUMMARY OF CHAPTER VII}

\section{Definitions.}

(1) Plane Section of a Surface - the points common to a surface and an intersecting plane. $\S 468$.

(2) Closed Surface - a surface such that every plane section of it consists of one or more closed lines. $\$ 469$.

(3) Polyhedron - a surface made up of intersecting planes. §470.

(4) Convex Polyhedron - one which lies wholly on one side or the other of each of its faces. $\$ 470$.

(5) Tetrahedron, Pentahedron, Hexahedron, Octahedron, Dodecahedron, Icosahedron. See $\$ 470$.

(6) Prism - a polyhedron of which two faces are convex polygons lying in parallel planes and identically equal, while the remaining faces are parallelograms. $\$ 471$.

(7) Right Prism - one whose lateral edges are perpendicular to its bases. $\$ 472$.

(8) Right Section of a Prism - a section made by a plane perpendicular to the lateral edges. $\$ 473$.

(9) Lateral Area of a Prism-the sum of the areas of the lateral faces. $\S 474$.

(10) Altitude of a Prism-the perpendicular distance between its bases. $\$ 475$.

(11) Truncated Prism - a polyhedron having parallel lateral edges like a prism, but bases which are neither parallel nor equal. $\S 483$.

(12) Volume of a Polyhedron - the space enclosed by the polyhedron, or the measure of that space. $\$ \S 485,502$.

(13) Parallelepiped - a prism whose bases are parallelograms. $\$ 489$.

(14) Right Parallelepiped - one in which a set of lateral edges is - perpendicular to the bases. $\$ 490$.

(15) Rectangular Parallelepiped - a right parallelepiped whose bases are rectangles. $\S 491$.

(16) Cube - a parallelepiped whose faces are all squares. $\S 492$.

(17) Pyramid - a polyhedron one of whose faces is a polygon, and the remaining faces are triangles having a common vertex. $\S 509$.

(18) Altitude of a Pyramid - the perpendicular distance from the vertex to the base. $\S 509$.

(19) Regular Pyramid-one whose base is a regular polygon and whose vertex lies on the perpendicular to the base drawn from its centre. $\S 510$. 
(20) Slant Height of a Regular Pyramid - the altitude of any of its lateral faces. $\$ 510$.

(21) Truncated Pyramid - the figure determined by the base and any section of a pyramid and the portions of the lateral faces intercepted between them. $\$ 511$.

(22) Frustum of a Pyramid - a truncated pyramid whose bases are parallel. $\$ 511$.

(23) Similar Polyhedrons - two polyhedrons which have the same number of faces similar, each to each, and similarly placed, and have their corresponding polyhedral angles equal. $\$ 530$.

(24) Regular Polyhedron - one whose faces are equal regular polygons, and whose polyhedral angles are all equal. $\$ 537$.

2. Axioms.

(1) If two figures are identically equal, their volumes are equal (Axiom 13). $\$ 486$.

(2) If equal volumes be added to equal volumes, or to the same volume, their sums are equal (Axiom 14). $\$ 487$.

3. Theorems on the Equality of Prisms.

(1) Two prisms, or truncated prisms, are identically equal if the three faces forming a trihedral angle in one are identically equal to the three faces forming a trihedral angle in the other, and are similarly placed. $\S \S 481,484$.

(2) Two right prisms are identically equal if their bases are identically equal and they have equal altitudes. $§ 482$.

4. Theorems on the Sections of Prisms and Pyramids.

(1) The sections of a prism made by parallel planes are polygons which are identically equal. $§ 476$.

(2) If a pyramid is cut by a plane parallel to its base, the edges and the altitude are divided in the same ratio, the section made by the plane is similar to the base, and the area of the section is to the area of the base as the squares of their distances from the vertex. $\$ 513$.

(3) If two pyramids have equal altitudes and bases of equal areas, the areas of sections made by planes equidistant from their vertices are equal. $\S 516$.

5. Theorems on tife Lateral Areas of Prisms and Prramids.

(1) The lateral area of a prism is equal to the product of a lateral edge and the perimeter of a right section of the prism. $\$ 479$. 
(2) The lateral area of a right prism is equal to the product of its altitude and the perimeter of its base. $\S 480$.

(3) The lateral area of a regular pyramid is equal to one-half the product of the perimeter of the base and the slant height. $\S 512$.

\section{Theorems on the Volume of a Prism.}

- (1) An oblique prism is equal in volume to a right prism whose base is a right section of the oblique prism and whose altitude is equal to a lateral edge of the oblique prism. $\S 488$.

(2) The volumes of two rectangular parallelepipeds having identically equal bases are in the same ratio as their altitudes. $\$ 496$.

(3) The volumes of two rectangular parallelepipeds having equal altitudes are in the same ratio as the areas of their bases. $\S 499$.

(4) The volume of any rectangular parallelepiped is the product of its altitude and the area of its base. $\$ 504$.

(5) The volume of any parallelepiped is equal to the product of its altitude and the area of its base. $\$ 505$.

(6) The volume of a prism is equal to the product of its altitude and the area of its base. $\$ \S 506,507$.

(7) The volume of a truncated triangular prism is equal to the sum of the volumes of three pyramids whose common base is one base of the prism and whose vertices are the three vertices of the other base. $\S 527$.

(8) The volume of a truncated right triangular prism is equal to the product of the area of that face to which the edges are perpendicular and one-third the sum of the lateral edges. $\$ 528$.

(9) The volume of any truncated triangular prism is equal to the product of the area of a right section and one-third the sum of the lateral edges. $§ 529$.

\section{Theorems on the Volume of a Pyramid.}

(1) If two triangular pyramids have equal altitudes and equal bases, they have equal volumes. $\S 517$.

(2) The volume of a triangular pyramid is one-third the volume of a triangular prism of the same base and altitude. $\$ 521$.

(3) The volume of a triangular pyramid is equal to one-third the product of its altitude and the area of its base. $\S 522$.

(4) The volume of any pyramid is equal to one-third the product of its altitude and the area of its base. $\$ 523$. 
(5) The volumes of any two pyramids are in the same ratio as the products of their altitudes and the areas of their bases. $\$ 524$.

(6) The volumes of two pyramids having equal altitudes are in the same ratio as the areas of their bases, or having equal bases are in the same ratio as their altitudes. $\$ 525$.

(7) The volumes of two tetrahedrons having a trihedral angle of the one equal to a trihedral angle of the other are in the same ratio as the products of the edges which meet in the vertices of these angles. $\$ 526$.

\section{Theorems on Similar Polyhedrons.}

(1) Any two edges or diagonals of a polyhedron are in the same ratio as the homologous edges or diagonals of a similar polyhedron. $\S 531$.

(2) The surfaces of two similar polyhedrons (i.e. the sums of the areas of all the faces) are in the same ratio as the squares of any two homologous edges or homologous diagonals. $\$ 532$.

(3) Two tetrahedrons are similar if the three faces meeting in a vertex of the one are similar and similarly situated to the three faces meeting in a vertex of the other. $\$ 533$.

(4) The volumes of two similar tetrahedrons are in the same ratio as the cubes of their homologous edges. $\$ 535$.

(5) The volumes of any two similar polyhedrons are in the same ratio as the cubes of two homologous edges. $\$ .536$.

\section{Miscellaneous 'Theorems.}

(1) The opposite lateral faces of a parallelepiped are parallel and identically equal. $\$ 493$.

(2) Any two opposite faces of a parallelepiped may be taken as the bases. $\$ 494$.

(3) The plane passed through two diagonally opposite edges of a parallelepiped divides it into two triangular prisms which are equal in volume. $\$ 495$.

(4) In any polyhedron the number of edges increased by two is equal to the number of faces together with the number of vertices (Euler's Theorem). §541.

(5) The sum of the face angles of any polyhedron together with eight right angles is equal to four times as many right angles as the polyhedron has vertices. $\S 542$. 


\section{CHAPTER VIII}

\section{CYLINDERS AND CONES}

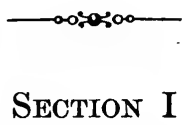

CYLINDERS

543. Definitions. If a straight line moves so as always to remain parallel to a fixed straight line, while some point of it traverses a fixed curve not in a plane with the fixed line, it is said to describe a cylindrical surface.

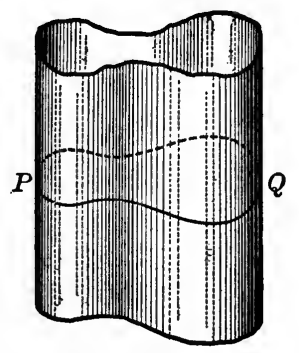

Cylindrical surface

Each position of the moving line is parallel to its former position, hence the line is said to move parallel to itself.

The moving line is called the generator of the cylindrical surface, and the guiding curve, the director.

$$
2 \mathrm{~A} \quad 353
$$


The generator in any position is an element of the surface.

A closed cylindrical surface is one whose section, made by any plane cutting all of its elements, is a closed curve.

544. A cylinder is a figure consisting of two parallel plane surfaces and a closed cylindrical surface intercepted between them.

The two plane surfaces are called the bases, and the cylindrical surface, the lateral surface of the cylinder. Through every point of the lateral surface there passes an element of the surface.

A circular cylinder is one whose bases

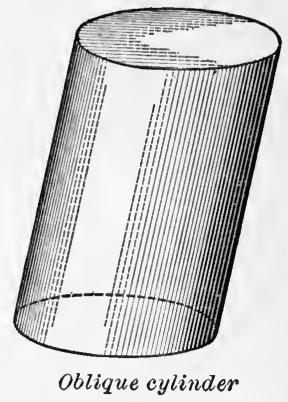
are circular.

A cylinder is called a right cylinder when the elements of its lateral surface are perpendicular to the bases; otherwise it is an oblique cylinder.

545. A right circular cylinder may be generated by revolving a rectangle about one of its sides. The opposite side will then generate the cylindrical surface, and the adjacent sides will generate the bases.

For this reason a right circular cylinder is sometimes called a cylinder of revolution.

The side of the rectangle about which the rotation takes place is called the axis of the cylinder.

546. A right section of a cylinder is the section made by a plane perpendicular to the elements of the lateral surface.

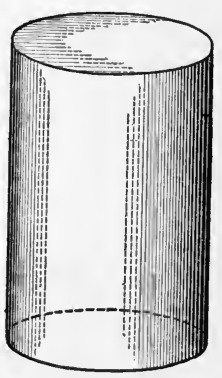

Right cylinder

The altitude of a cylinder is the perpendicular distance between its bases. 
547. Theorem I. If a plane contains one element of the lateral surface of a cylinder and meets the lateral surface at any other point, it contains also a second element, and the section of the cylinder by the plane is a parallelogram.

If the plane $L$ contains the element $A B$ of the lateral surface of the cylinder $P Q$, and meets the lateral surface again at $D$, it must contain also the element through $D$, since that element is parallel to $A B$, and must lie in a plane with $A B$.

The plane $L$ therefore cuts the lateral surface of the cylinder along two parallel lines $A B$ and $C D$, and the bases along two straight lines $A D$ and $B C$ which are also parallel (Art. 419).

Hence the section of the cylinder made by the plane is a parallelogram.

548. Corollary. The section of a right cylinder made by a plane containing an element of the lateral surface is a rectangle.

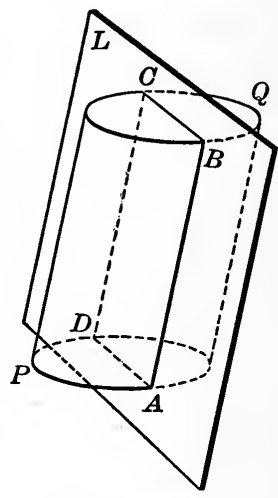

549. Definition. If a plane contains one element of the lateral surface of a cylinder, and only one, it is said to be tangent to the cylinder.

The element which the tangent plane contains is called the element of contact.

A straight line is tangent to a cylinder when it meets an element of the lateral surface, and lies in the tangent plane containing that element.

550. Theorem II. The plane determined by a tangent to any circular section of a cylinder and the element of the lateral surface passing through its point of contact is tangent to the cylinder.

For if it is not tangent to the cylinder it must contain a second element of the lateral surface, and so contain a chord of the section instead of the tangent to the section.

Conversely. If a plane is tangent to a circular cylinder it intersects the plane of the base in a tangent to the base. 
551. Definition. If sections of a cylindrical surface are made by two planes, those are corresponding points of the sections which lie upon the same element of the surface.

\section{Proposition I}

552. Parallel sections of a cylindrical surface are identically equal.

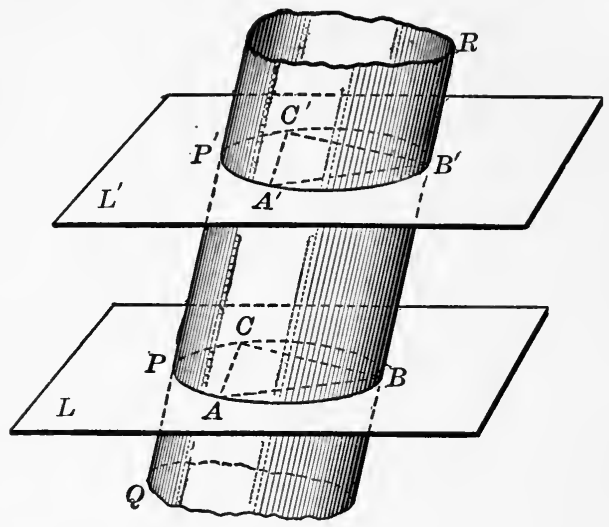

Let $Q R$ be any cylindrical surface of which $P A B C$ and $P^{\prime} A^{\prime} B^{\prime} C^{\prime}$ are parallel sections.

It is required to prove that $P A B C$ and $P^{\prime} A^{\prime} B^{\prime} C^{\prime}$ are identically equal.

Proof. Suppose $A, A^{\prime} ; B, B^{\prime} ; C, C^{\prime}$ are any three pairs of corresponding points in the two sections.

Then, $A A^{\prime}$ and $B B^{\prime}$ are not only parallel but also equal (Art. 420 ), and hence $A B B^{\prime} A^{\prime}$ is a parallelogram. (Art. 127.)

Therefore

$$
\begin{aligned}
& A B=A^{\prime} B^{\prime} . \\
& A C=A^{\prime} C^{\prime}, \text { and } \\
& B C=B^{\prime} C^{\prime} .
\end{aligned}
$$

Similarly, 
Therefore $\triangle \mathrm{s} A B C$ and $A^{\prime} B^{\prime} C^{\prime}$ are identically equal, and can be superposed.

(Art. 53.)

Suppose now that the section $P^{\prime} A^{\prime} B^{\prime} C^{\prime}$ is placed upon the section $P A B C$ so that the points $A^{\prime}, B^{\prime}, C^{\prime}$ coincide with their corresponding points $A, B, C$, and let $A, A^{\prime}$ and $C, C^{\prime}$ remain fixed while $B$ traverses the section $P A B C$. Since $B$ will always coincide with its corresponding point $B^{\prime}$, every point of the one section will coincide with its corresponding point in the other.

Therefore the two sections are identically equal.

553. Corollary I. The bases of a cylinder are identically equal.

554. Corollary II. Any two right sections of a cylinder are identically equal.

555. Corollary III. Any section of a cylinder parallel to the base is identically equal to the base.

556. Corollary IV. All sections of a circular cylinder parallel to its bases are equal circles, and the straight line joining the centres of the bases passes through the centres of all the parallel sections.

\section{EXERCISES}

1. If through any point of the lateral surface of a cylinder a straight line is drawn parallel to any element of the surface, this straight line is also an element of the surface.

2. Show that all the elements of the lateral surface of a cylinder are of equal lengths.

3. The line of intersection of two planes tangent to a cylinder is parallel to an element of the lateral surface, and to the plane through their two elements of contact.

4. Find the locus of points (1) at a given distance from a given straight line; (2) at given distances from each of two given parallel straight lines. 


\section{Definitions}

557. A prism is inscribed in a cylinder when its bases and the bases of the cylinder lie in the same planes, and its lateral edges are elements of the lateral surface of the cylinder.

The cylinder is at the same time circumscribed about the prism.

The section of an inscribed prism made by any plane is inscribed in the section of the cylinder made by the same plane.

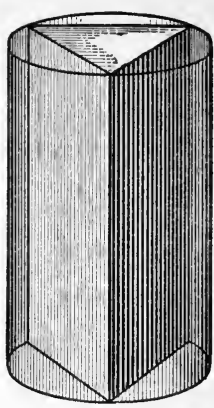

Prism inscribed in a cylinder

558. A prism is circumscribed about a cylinder when its bases and the bases of the cylinder lie in the same planes, and its lateral faces are tangent to the cylinder.

The cylinder is at the same time inscribed in the prism.

The section of a circumscribed prism made by any plane is circumscribed about the section of the cylinder made by the same plane.

559. If a regular prism is inscribed, or circumscribed, to a circular cylinder, and the number of its lateral faces

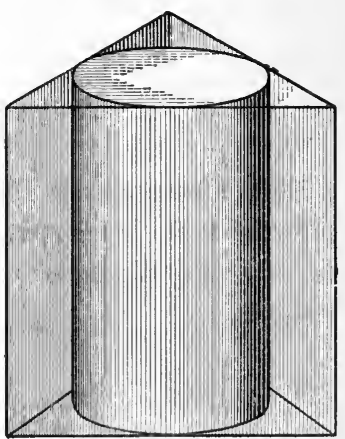

Prism circumscribed about a cylinder is indefinitely increased in some regular way, the lateral surface of the prism approaches the lateral surface of the cylinder as its limit, the volume of the prism approaches the volume of the cylinder as its limit, and the perimeter and area of a right section of the prism approach the perimeter and area of a right section of the cylinder as their limits. 


\section{Proposition II}

560. The latercul area of a circular cylinder is equal to the product of the perimeter of a right section of the cylinder and the length of an element of its lateral surface.
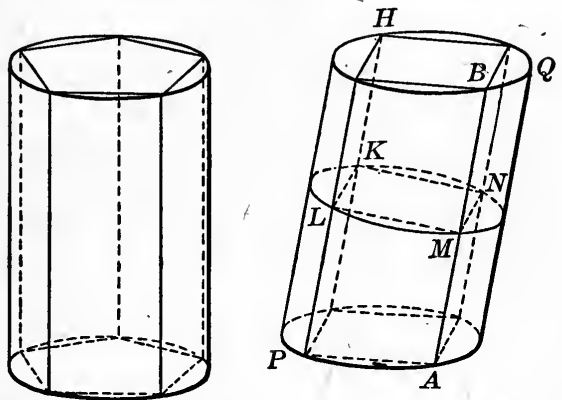

Let $P Q$ be a circular cylinder of which $S$ is the perimeter of a right section, and $A B$ an element of the lateral surface.

It is required to prove that the lateral area of $P Q$ is equal to $S$ times $A B$.

Proof. In the cylinder $P Q$ inscribe a regular prism $A H$, one of whose elements is $A B$. Let $K M$ be a right section of this prism. The lateral area of $A H=$ perimeter of $K M \times A B$.

(Art. 479.)

If the number of lateral faces of $A H$ is indefinitely increased, the lateral area of $A H$ approaches the lateral area of $P Q$ as its limit, and the perimeter of $K M$ approaches $S$ as its limit.

Therefore the lateral area of $P Q=S \times A B$.

(Art. 230.)

561. CoRollarx. The lateral area of a right circular cylinder is equal to the product of the altitude and the circumference of the base.

If the lateral area is represented by $A$, the radius of the base by $r$, and the altitude by $h$,

$$
A=2 \pi r h \text {. }
$$




\section{Proposition III}

562. The volume of a circular cylinder is equal to the product of its altitude and the area of its base.

The proof of this theorem is similar to that of Proposition II, and is left to the pupil. Reference should be made to Article 507.

If the volume is represented by $V$, the altitude by $h$, and the radius of the base by $r$,

$$
V=\pi r^{2} h .
$$

563. Corollary. The volumes of all circular cylinders, whether right or oblique, having equal bases and equal altitudes are equal.

\section{EXERCISES}

Note. Use $3 \frac{1}{7}$ as the approximate value of $\pi$.

1. Find the volume, the lateral area, and the total area of a right circular cylinder the diameter of whose base is 14 inches, and whose altitude is 11 inches.

2. The lateral area of a right circular cylinder is 528 square feet, and its volume is 1584 cubic feet. Find the diameter and circumference of its base, and its altitude.

3. Two right circular cylinders are of equal height while the circumference of one is double the circumference of the other. What is the ratio of their lateral areas? Also of their volumes?

4. A hollow cylindrical iron tube has an outer diameter of 12 inches and an inner diameter of 9 inches. How many cubic inches of iron are there in a piece of the tube 2 feet long?

5. When a tap is opened the water in a pipe of one inch inner diameter flows at the rate of two miles an hour. How many gallons of water would flow from the tap in 20 minutes?

Take $7 \frac{1}{2}$ gallons to a cubic foot.

6. Show that the volumes of two similar right circular cylinders are in the same ratio as the cubes of their altitudes, or as the cubes of the radii of their bases.

7. What is the altitude of a right circular cylinder if its lateral area equals the sum of the areas of its bases ? 


\section{SeCtion II}

\section{THE CONE}

564. Definition. If a straight line moves so as always to pass through a fixed point, while some point of the line traverses a fixed curve not in a plane with the fixed point, it will describe a conical surface.

As in the cylindrical surface, the generating line in any position is an element of the surface.

The directing curve will always be considered a closed curve.

The fixed point through which the generating line always passes is called the vertex of the surface.

If the generating line is indefinite in length, the surface is divided into two parts at the ver-

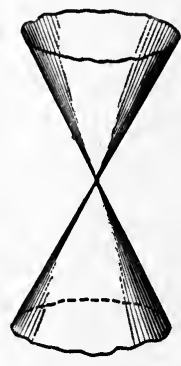

Conical surface tex, called the two sheets, or the two nappes of the conical surface.

When we speak of a plane section of a conical surface, we shall always have in mind the section made by a plane which cuts all the elements on the same side of the vertex, that is, a section of one of the sheets.

565. Definition. A cone is a figure consisting of a plane surface and a conical surface intercepted between the plane surface and the vertex.

The plane surface is called the base, and the conical surface the lateral surface. The vertex of the conical surface is the vertex of the cone.

The straight line joining any point of the lateral surface to the vertex is an element of the lateral surface, since it must coincide with the generating line in one position.

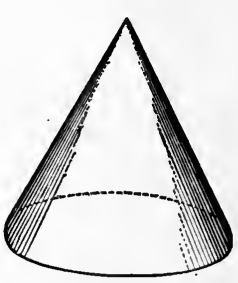

Circular cone

A circular cone is one whose base is circular. The straight line joining the vertex to the centre of the base is called the axis of the cone. 
When the vertex lies on the perpendicular to the base drawn from its centre, the cone is called a right circular cone.

566. The altitude of a cone is the perpendicular distance from the vertex to the plane of the base.

567. The slant height of a right circular cone is the length of an element of the lateral surface.

568. Theorem I. Any plane which contains one element of the lateral surface of a cone and meets the surface at any other point, contains also a second element, and the section of the cone by the plane is a triangle.

If the plane $L$ contains the element $S B$ of the cone $S-A B C$, and meets the surface again at the point $D$ it must contain the element $S D$, since it contains two points of this element.

The section of the cone by the plane $L$ therefore consists of the line-segments $S B, S D$, and $B D$, and is thus a triangle.

569. Corollary. The section of a right circular cone by a plane through the vertex is an isosceles triangle.

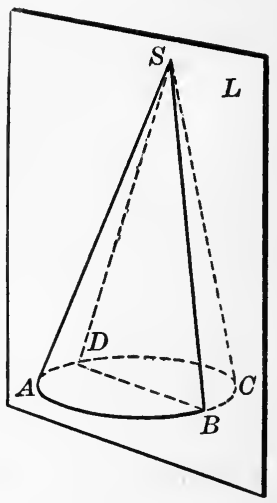

570. Definition. If a plane contains one element of the lateral surface of a cone and only one, it is said to be tangent to the cone, and the element which it contains is called the element of contact.

571. Theorem II. The plane determined by a tangent to the base of a circular cone and the element of the lateral surface passing through its point of contact is tangent to the cone.

Conversely. If a plane is tangent to a circular cone, its intersection with the plane of the base is tangent to the base.

See Article 550. 


\section{Proposition IV}

572. Any section of a circular cone made by a plane parallel to the base is a circle, and its centre lies upon the straight line joining the vertex to the centre of the base.

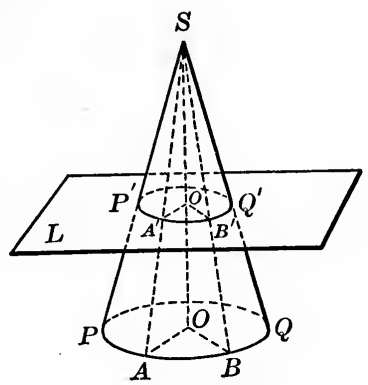

Let $A^{\prime} B^{\prime} Q^{\prime}$ be the section of the circular cone $S-A B Q$ made by a plane $L$ parallel to the base, and let $S O$ be the straight line joining $S$ to the centre of the base.

It is required to prove that $A^{\prime} B^{\prime} Q^{\prime}$ is a circle whose centre lies on $S O$.

- Proof. Let $A^{\prime}$ and $B^{\prime}$ be any two points of the section, and let the planes determined by the line $S O$ and $A^{\prime}, S O$ and $B^{\prime}$ intersect the lateral surface of the cone in the straight lines $S A^{\prime} A, S B^{\prime} B$, the base in the lines $A O, B O$, and the plane $L$ in the lines $A^{\prime} O^{\prime}, B^{\prime} O^{\prime}$, parallel to $A O$ and $B O$, respectively.

Therefore $\quad \frac{O^{\prime} A^{\prime}}{O A}=\frac{O^{\prime} S}{O S}=\frac{O^{\prime} B^{\prime}}{O B}$.

But $O A=O B$. Therefore $O^{\prime} A^{\prime}=O^{\prime} B^{\prime}$.

Since $A^{\prime}$ and $B^{\prime}$ are any points whatever in the section made by $L$, all points of the section are equidistant from $O^{\prime}$.

Therefore the section is a circle with its centre at $O^{\prime}$.

573. Conollary. The radii of two sections of a circular cone, parallel to the base, are in the same ratio as their distances from the vertex. 
574. Definitions. A pyramid is inscribed in a cone when their bases lie in the same plane, and the lateral edges of the pyramid are elements of the lateral surface of the cone.

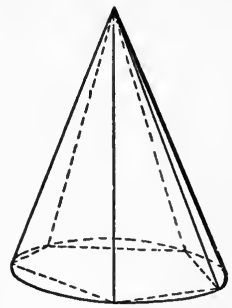

Pyramid inscribed in a cone

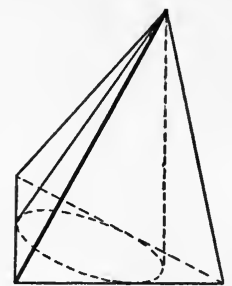

Pyramid circumscribed about a cone

The vertex of the inscribed pyramid coincides with the vertex of the cone, and the base of the pyramid is inscribed in the base of the cone. The section of the inscribed pyramid made by any plane is inscribed in the section of the cone made by the same plane.

575. A pyramid is circumscribed about a cone when their bases lie in the same plane, and the lateral faces of the pyramid are tangent to the cone.

The vertex of the circumscribed pyramid coincides with the vertex of the cone, and the base of the pyramid is circumscribed about the base of the cone. The section of the circumscribed pyramid made by any plane is circumscribed about the section of the cone made by the same plane.

576. If a regular pyramid is inscribed, or circumscribed, to a circular cone and the number of its lateral faces is indefinitely increased in some regular way, the lateral surface of the pyramid approaches the lateral surface of the cone as its limit, and the volume of the pyramid approaches the volume of the cone as its limit. 


\section{Proposition V}

577. The lateral area of a right circular cone is equal to one-half the product of the circumference of the base and the slant height.

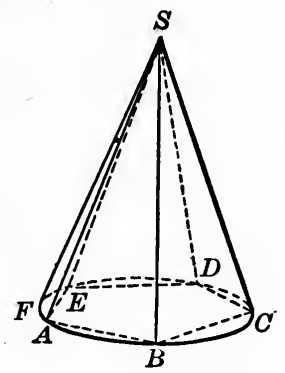

Let $S-A C E$ be a right circular cone, of which $l$ is the slant height, and $M$ the circumference of the base.

It is required to prove that the lateral area of $S-A C E$ is equal to $\frac{1}{2} M l$.

Proof. Inscribe in the cone $S-A C E$ a regular pyramid $P$, one of whose elements is $S A=l$, and the perimeter of whose base is $H$.

Then the lateral area of $P=\frac{1}{2} \mathrm{Hl}$.

(Art. 512.)

If the number of lateral faces of $P$ is indefinitely increased, the lateral area of $P$ approaches the lateral area of $S-A C E$ as its limit, and the perimeter $H$ approaches the circumference $M$ as its limit.

Therefore the lateral area of $S-A C E=\frac{1}{2} M l$.

(Art. 230.)

If the radius of the base is $r$, and the lateral area is represented by $A$,

$$
A=\pi r l \text {. }
$$

578. Definition. The frustum of a cone is that portion of a cone intercepted between the base and a plane parallel to the base intersecting the lateral surface. 


\section{Proposition VI}

579. The volume of a circular cone is equal to onethird the product of its altitude and the area of its base.

The proof of this theorem is similar to that of Proposition V, and is left to the pupil.

If the volume is represented by $V$, the altitude by $h$, and the radius of the base by $r$,

$$
V=\frac{1}{3} \pi r^{2} h \text {. }
$$

\section{MISCELLANEOUS EXERCISES}

1. Find the lateral area and the volume of a right circular cone, the area of whose base is 154 square inches and whose altitude is 11 inches.

2. The slant height of a right circular cone is 4 metres. How far from the vertex must a section parallel to the base be taken so as to divide the lateral area into two equal parts?

3. A right triangle whose sides are 3, 4, 5 feet, respectively, is rotated about the shortest side. Find the area of the surface described by the hypotenuse.

4. Show that the lateral areas of similar right circular cylinders are in the same ratio as the squares of their altitudes, or as the squares of the radii of their bases.

Definition. Similar right circular cylinders are generated by the rotation of similar rectangles about homologous sides:

5. Show that the lateral areas of two similar cones of revolution are in the same ratio as the squares of their slant heights, or as the squares of their altitudes, or as the squares of the radii of their bases.

Definition. Similar cones of revolution, or right circular cones, are generated by similar right triangles rotating about homologous sides.

6. Show that the volumes of two similar right circular cones are in the same ratio as the cubes of their altitudes, or as the cubes of the radii of their bases.

7. The volumes of two similar cones of revolution are in the ratio of 512.729 . What is the ratio of their lateral areas? 
8. Show that the lateral area of the frustum of a right circular cone is equal to the sum of the circumferences of its bases multiplied by one-half the slant height.

9. Show that the lateral area of the frustum of a right circular cone is equal to the circumference of a section midway between the bases multiplied by the slant height.

10. Show that the volume of the frustum of a circular cone, the areas of whose bases are $B$ and $b$ and whose altitude is $h$, is given by the formula

See Ex. 2, p. 338.

$$
V=\frac{1}{3} h(B+b+\sqrt{B b}) .
$$

11. A right circular cylinder of height $2 \mathrm{ft}$. and the radius of whose base is 6 in. rolls on a plane making one complete revolution. What is the shape of the surface covered by it? Find its area.

12. A right circular cone whose altitude is $12 \mathrm{in}$. and the radius of whose base is 5 in. rolls on a plane, without slipping, making one complete revolution. What is the shape of the surface covered? Find its area.

13. A regular hexagonal prism is inscribed in a right circular cylinder. Compare their lateral areas and their volumes.

14. The base of a right circular cylinder has a radius of $7 \mathrm{~cm}$. and an altitude of $7 \mathrm{~cm}$. Find its total surface area and its volume.

15. A rectangle whose adjacent sides are $5 \mathrm{ft}$. and $7 \mathrm{ft}$., respectively, revolves in succession about these sides; show that the volumes of the cylinders generated are in the ratio $7: 5$.

16. The total surface of a right circular cone is $462 \mathrm{sq} . \mathrm{cm}$., and the slant height is twice the radius of the base; find the volume.

17. In a right circular cylinder of height $3 \mathrm{ft}$. and $8 \mathrm{in}$. radius, a square prism is inscribed. Find its volume.

18. A square whose side is $50 \mathrm{~cm}$. revolves about one of its diagonals ; find the area and volume of the figure so generated.

19. Show that in a right circular cone all the elements of the lateral surface are equal in length.

20. Show that the total area $T$ of a right circular cylinder, including the areas of the two bases, is given by the formula :

$$
T=2 \pi r h+2 \pi r^{2}=2 \pi r(h+r) .
$$

21. A circular cistern is $22 \mathrm{ft}$. in circumference at the top, $16 \mathrm{ft}$. at the bottom, and $8 \mathrm{ft}$. deep. How many gallons of water will it hold, assuming $7 \frac{1}{2}$ gallons to the cubic foot? 


\section{Definitions.}

\section{SUMMARY OF CHAPTER VIII}

(1) Cylindrical Surface - the surface described by a straight line moving parallel to a fixed straight line, while some point of it traverses a fixed curve not in a plane with the fixed line. $\$ 543$.

(2) Conical Surface - the surface described by a straight line which moves so as always to pass through a fixed point, while some point of it traverses a fixed curve not in a plane with the fixed point. $\$ 564$.

(3) Closed Cylindrical Surface - one for which the directing curve is closed. $\$ 543$.

(4) Vertex of a Conical Surface - the point through which all the elements of the surface pass. $\$ 564$.

(5) Cylinder - a figure consisting of two parallel plane surfaces and a closed cylindrical surface intercepted between them. $\S 544$.

(6) C'ircular Cylinder - one whose bases are circular. $\$ 544$.

(7) Right Cylinder - one in which the elements of the lateral surface are perpendicular to the bases. $\$ 544$.

(8) Cone - a figure consisting of a plane and the conical surface intercepted between it and the vertex. $\$ 565$.

(9) Circular Cone - one whose base is circular. $\$ 565$.

(10) Right Circular Cone - one whose vertex lies on the perpendicular to the base drawn from its centre. $\$ 565$.

(11) Tangent to a Cylinder or a Cone - a plane containing one element of the lateral surface, and only one; also, a line meeting one element of the surface, and only one. $\$ 549$. $\$ 570$.

(12) Frustum of a Cone - that portion of a cone intercepted between its base and a plane parallel to the base, intersecting the lateral surface. $\$ 578$.

\section{Theorems on the Properties of Cylinders.}

(1) A plane which contains one element of the lateral surface of a cylinder in general contains also another element, and the section is a parallelogram. $\$ 547$.

(2) The section of a right cylinder made by a plane containing an element of the lateral surface is a rectangle. $\$ 548$.

(3) The plane determined by a tangent to any circular section of a cylinder and the element of the lateral surface passing through its point of contact is tangent to the cylinder ; and conversely, if a plane is tangent to a circular cylinder it intersects the plane of the base in a tangent to the base. $\S 550$. 
(4) Parallel sections of a cylindrical surface are identically equal. $\S 552$.

(5) The bases of a cylinder are identically equal. $§ 553$.

(6) Any two right sections of a cylinder are identically equal. $\$ 554$.

(7) Any section of a cylinder parallel to the base is identically equal to the base. $\S 555$.

(8) All the sections of a circular cylinder parallel to its bases are equal circles, and the straight line joining the centres of the bases passes through the centres of all the parallel sections. $\S 556$.

3. Theorems on the Properties of Cones.

(1) Any plane containing one element of the lateral surface of a cone contains in general a second element and the section of the cone by the plane is a triangle. $\$ 568$.

(2) The plane determined by a tangent to the base of a circular cone and the element of the lateral surface passing through its point of contact is tangent to the cone ; and conversely, if a plane is tangent to a circular cone, its intersection with the plane of the base is tangent to the base. $\$ 571$.

(3) Any section of a circular cone made by a plane parallel to the base is a circle, and its centre lies upon the straight line joining the vertex to the centre of the base. $\$ 572$.

4. Theorems on the lateral Area and the Volume of a Cylinder.

(1) The lateral area of a circular cylinder is equal to the product of the perimeter of a right section of the cylinder and the length of an element of its lateral surface. $\$ 560$.

(2) The lateral area of a right circular cylinder is equal to the product of the circumference of the base and the altitude. $A=2 \pi r h$. $\S 561$.

(3) The volume of a circular cylinder is equal to the product of its altitude and the area of its base. $V=\pi r^{2} h . \quad \S 562$.

(4) The volumes of all circular cylinders, whether right or oblique, having equal bases and equal altitudes are equal. $§ 563$.

5. Theorems on the lateral Area and the Volume of a Cone.

(1) The lateral area of a right circular cone is equal to one-half the product of the circumference of the base and the slant height. $A=\pi r h . \quad \S 577$.

(2) The volume of a circular cone is equal to one-third the product of its altitude and the area of its base. $V=\frac{1}{3} \pi r^{2} h . \quad \S 579$. 2 B 


\section{CHAPTER IX}

\section{THE SPHERE}

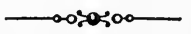

\section{SECTION I}

\section{PLANE SECTIONS AND TANGENT PLANES}

580. Definitions. A sphere is a closed surface such that all points of it are equidistant from a fixed point within it.

The fixed point is called the centre of the sphere.

If a circle is rotated about its diameter, the surface described by it is a sphere.

Any straight line drawn from the centre to a point on the sphere is called a radius of the sphere.

A straight line drawn through the centre and terminated both ways by the sphere is called a diameter of the sphere.

The length of a diameter is twice the length of a radius.

It follows from the definition of a sphere that all radii of the same sphere are equal, and hence that all diameters of the same sphere are equal.

581. Theorem. Equal spheres have equal radii and equal diameters.

Conversely. Spheres which have equal radii or equal diameters are equal.

582. Spheres which have the same centre but do not coincide are called concentric spheres.

Spheres having the same centre and one point in common must coincide throughout. 


\section{Proposition I}

583. The section of a sphere made by a plane is a circle.

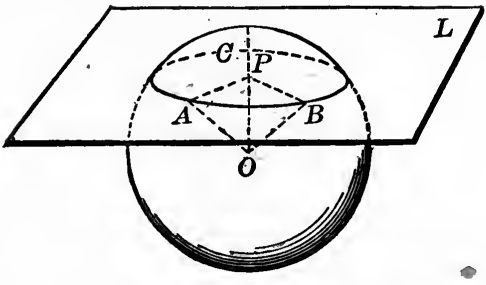

Let $O$ be the centre of the given sphere and $A, B, C$ three points on the section made by any plane $L$.

It is required to prove that $A B C$ is a circle.

Proof. From $O$ draw $O P$ perpendicular to the plane $L$, draw the radii $O A$ and $O B$, and join $P A$ and $P B$.

$\triangle \mathrm{s} O P A$ and $O P B$ are identically equal.

\section{Hence

$$
P A=P B \text {. }
$$

But $A$ and $B$ are any two points common to the plane and sphere.

Therefore all points common to the plane and sphere are equidistant from $P$.

Therefore the section of the sphere made by the plane $L$ is a circle.

584. Corollary I. Sections of a sphere made by planes equidistant from the centre are equal circles, and of two sections made by planes unequally distant from the centre, that is the greater circle which is made by the nearer plane.

585. Definition. Any circle lying on a sphere is called a circle of the sphere.

A circle whose plane passes through the centre of the sphere is called a great circle of the sphere; one whose plane does not pass through the centre is called a small circle of the sphere. 
A quadrant is one quarter of a great circle of a sphere, and subtends a right angle at the centre of the sphere.

586. CoROLLARY II. The centre of any circle of a sphere is the foot of the perpendicular drawn from the centre of the sphere to the plane of the circle.

587. Corollary III. The centres of all great circles coincide with the centre of the sphere, and all great circles on the same sphere are equal.

588. Coroliary IV. Every great circle divides the sphere into two equal parts.

589. Corollary V. Any two great circles on the same sphere bisect each other.

Their planes intersect in a diameter of each circle.

590. Corollary VI. An are of a great circle can be drawn through any two given points on a sphere, and if the two given points are not the extremities of a diameter, only one such arcless than a semicircle can be drawn.

The two given points and the centre of the sphere determine the plane of the great circle.

591. Corollary VII. Through three given points on a sphere one and only one circle can be drawn.

592. Definition. The diameter of a sphere perpendicular to the plane of any circle of the sphere is called the axis of that circle, and the extremities of the diameter are called the poles of the circle.

Are the poles of a circle of a sphere equally or unequally distant from the plane of the circle?

593. By the distance between two points on a sphere is meant the length of the arc, not greater than a semicircle, of the great circle passing through them.

A distinction is made here between the distance measured on a sphere and measured along a straight line. 


\section{Proposition II}

594. All points of any circle of a sphere are equidistant from either of its poles.

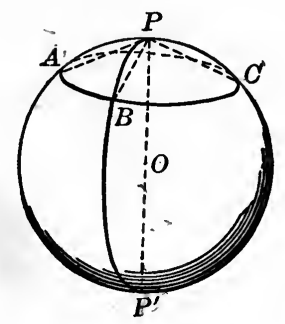

Let $A, B, C$ be any three points of a given circle of the sphere whose centre is $O$, and let $P$ and $P^{\prime}$ be the poles of that circle.

It is required to prove that $A, B, C$ are equidistant from $P$, and also equidistant from $P^{\prime}$.

Proof. If planes are passed through the line $P P^{\prime}$ and the points $A, B, C$, the arcs determined by them, $P A, P B, P C$, $P^{\prime} A, P^{\prime} B, P^{\prime} C$, are arcs of great circles. Hence the distance from $P$ to $A$ is the length of the arc $P A$, etc.

Since $P P^{\prime}$ is perpendicular to the plane of the circle $A B C$ at its centre, the chords $P A, P B, P C$ are all equal. Prove.

Therefore the arcs $P A, P B, P C^{\circ}$ are all equal.

Similarly, the $\operatorname{arcs} P^{\prime} A, P^{\prime} B, P^{\prime} C$ are all equal.

(Art. 163.)

595. Definition. The distance on a sphere of any point of a circle from its nearer pole is called the polar distance of the circle.

596. Corollary I. The polar distance of any point of a great circle is one-fourth the circumference of a great circle, or more briefly, is a quadrant. 
597. CoRollary II. If a point on a sphere is a quadrant's distance from each of two given points on the sphere, it is the pole of the great circle passing through the two points.

Let $P$ be a quadrant's distance from $A$ and $B$; through $P A, P B$, and $A B$ pass great circles. Then $\angle P O A$ is a right angle. Also $\angle P O B$ is a right angle. Therefore $P O$ is perpendicular to the plane $A O B$. (Art. 397.) Hence $P$ is the pole of the great circle through $A$ and $B$.

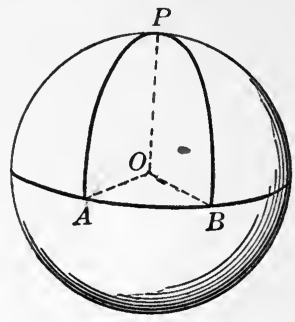

598. Corollary III. If one point on a sphere is a quadrant's distance from another, it is the pole of some great circle passing through the other.

599. The study of the geometry of a sphere, or Spherical Geometry, is greatly simplified by the use of a slated globe upon which chalk marks can be made and erased. If the length of the radius of such a spherical surface is known, Corollary II enables us to draw on it the arc of a great circle passing through any two given points.

To do this first draw in any plane a circle whose radius $r$ is equal to the radius of the given spherical surface, and make an opening in the compasses equal to $E F$, the chord of a quadrant.

Then, if $A$ and $B$ are the given points on the spherical surface, arcs described on the sphere, with the stationary arm of the com. passes at $A$ and $B$ in turn, will intersect at $P$, the pole of the great circle through $A$ and $B$.

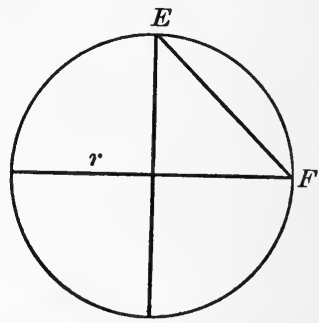
The arc described with the stationary arm at $P$, and with the same opening, will then be the arc of a great circle through $A$ and $B$.

By the same method we can find the pole of a given arc of a great circle, or draw the great circle of which a given point is the pole.

A geometrical method of finding the length of the diameter of a given material spherical solid will be found in Proposition XIX, page 393.

For all work on a sphere, a pair of compasses with curved arms will be found most convenient. 
600. Definitions. A plane which meets a sphere at one and only one point is tangent to the sphere. The point which the sphere and plane have in common is called the point of contact.

A straight line is tangent to a sphere when it meets the sphere at one and only one point, no matter how far it is produced.

A straight line tangent to a sphere is tangent to every section of the sphere made by planes through the line.

\section{Proposition III}

601. A plane which is perpendicular to a radius of a sphere at its extremity is tangent to the sphere.

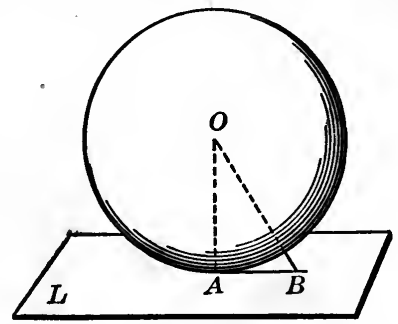

Let the plane $L$ be perpendicular to the radius $O A$ of the sphere whose centre is $O$, at its extremity $A$.

It is required to prove that the plane $L$ is tangent to the sphere.

Proof. First, the plane has the point $A$ in common with the sphere.

Next, every other point of $L$ lies outside of the sphere. Why?

Therefore the plane $L$ is tangent to the sphere.

Conversely. A plane which is tangent to a sphere is perpendicular to the radius drawn to its point of contact.

For if not, the perpendicular to it drawn from the centre is less than a radius, and the plane must pass inside the sphere. 
602. Corollary I. All lines tangent to a sphere at one point lie in a plane tangent to the sphere at that point.

For they are all perpendicular to the radius through the point. Why?

603. Corollary II. The plane of two straight lines tangent to a sphere at the same point is also tangent to the sphere at that point.

\section{Proposition IV}

604. The intersection of two spheres is a circle, the plane of which is perpendicular to the straight line joining the centres of the spheres, and the centre of which is on that line.

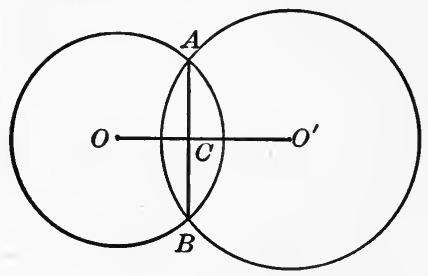

Let $O$ and $O^{\prime}$ be the centres of two spheres which intersect.

It is required to prove that their intersection is a circle whose plane is perpendicular to $O O^{\prime}$ and whose centre lies on $O O^{\prime}$.

Proof. Let $A$ be any point common to the two spheres.

Through $A$ and $O O^{\prime}$ pass a plane. This will cut the spheres in two circles, which intersect at $A$ and another point $B$.

The chord $A B$ is perpendicular to $O O^{\prime}$, and is bisected by it at $C$.

(Art. 168.)

If the plane of $O A O^{\prime}$ is revolved about $O O^{\prime}$, the two circles will describe the given spheres, and the point $A$ will trace their line of intersection.

Since $A C$ is perpendicular to $O O^{\prime}$ and remains of constant length, $A$ will describe a circle whose plane is perpendicular to $O O^{\prime}$ and whose centre is $C$. 


\section{Proposition V}

605. Through any four points not lying in the same plane, one and only one sphere can be passed.

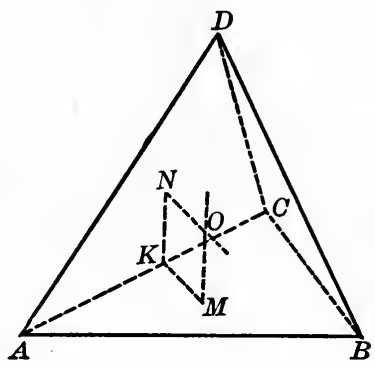

Let $A, B, C, D$ be four points not lying in the same plane.

It is required to prove that one and only one sphere can be passed through $A, B, C$, and $D$.

Proof. Pass planes through the points $A, B, C$ and $A, D, C$, and find the centres $M$ and $N$ of the circles circumscribing $\triangle \mathrm{s} A B C$ and $A D C$, respectively.

Let $K$ be the mid-point of the line-segment $A C$, and join $M K$ and $N K$.

These lines will be perpendicular to $A C$ at $K$.

(Art. 166.)

The plane $M K N$ is perpendicular to both planes $A B C$ and $A D C$, being perpendicular to their intersection $A C$. (Art. 438.)

The perpendiculars to the planes $A B C$ and $A D C$ erected at $M$ and $N$ will therefore lie in the plane $M K N$, and must intersect at some point $O$.

(Arts. 440, 98.)

Then $O$ is the centre of a sphere which passes through the four points $A, B, C, D$.

For, the straight line $M O$ is the locus of points equidistant from $A, B$, and $C$.

The straight line $N O$ is the locus of points equidistant from $A, D$, and $C$. 
Hence the point $O$ is equidistant from $A, B, C$, and $D$, and a sphere whose centre is $O$ and radius equal to $O A$ will pass through the four given points.

Also, since $M O$ and $N O$ have no other common point than $O$, no other sphere can be passed through the four given points.

606. Definition. A polyhedron is said to be inscribed in a sphere (or the sphere to be circumscribed about the polyhedron) when the vertices of the polyhedron lie on the sphere. A polyhedron is said to be circumscribed about a sphere (or the sphere to be inscribed in the polyhedron) when the faces of the polyhedron are tangent to the sphere.

607. Corollarx I. One and only one sphere can be circumscribed about a tetrahedron.

608. CoRollary II. The perpendiculars to the four faces of a tetrahedron, erected at the centres of the circumscribed circles, all pass through one point.

609. Corollary III. The six planes perpendicular to the edges of a tetrahedron at their mid-points have one point in common.

\section{EXERCISES}

1. If from a point outside of a sphere straight lines are drawn tangent to the sphere, the line-segments lying between the point and the points of contact are all equal.

2. Show that in the preceding example the points of contact all lie on a small circle of the sphere whose axis is the straight line joining the given point to the centre of the sphere.

3. Construct a sphere which will pass through the eight vertices of a cube.

4. Show that the sphere which is passed through two pairs of diagonally opposite vertices of a rectangular parallelepiped passes also through the other vertices. 


\section{Proposition VI}

610. One and only one sphere can be inscribed in any given tetrahedron.

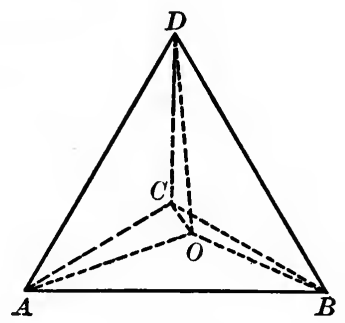

Let $A B C D$ be any given tetrahedron.

It is required to prove that one and only one sphere can be inscribed in $A B C D$.

The proof is left to the pupil.

Suggestion. Pass planes through the three edges of any one side, as $A B C$, bisecting the dihedral angles.

611. Corollary. The planes which bisect the six dihedral angles of any tetrahedron have one point in common.

\section{EXERCISES}

1. What is the locus of points at a distance $a$ from a point $A$, and at a distance $b$ from a point $B$ ?

2. What is the locus of points in space whose distances from two fixed points are in a given ratio? (See Prop. XX, Chap. III.)

3. What is the locus of the centres of the spheres to which three given intersecting planes are tangent?

4. If two spheres are tangent to the same plane at the same point, the straight line joining their centres passes through that point.

5. How many spheres can be made to touch four planes not all passing through the same point? 


\section{Section II}

\section{SPHERICAL ANGLES, TRIANGLES, AND POLYGONS}

612. Definition. The angle formed by any two intersecting circles is defined to be the angle formed by their tangents at their common point.

The same definition holds for the angle formed by any two intersecting curves.

The angle formed by two intersecting arcs of great circles of a sphere is called a spherical angle.

When the tangents to the two ares are at right angles the spherical angle is called a right spherical angle.

The point at which the two ares intersect is called the vertex of the spherical angle.

613. The planes of two great circles of a sphere intersect in a diameter. Hence the tangents of two intersecting ares of great circles at their common point are perpendicular to the edge of the dihedral angle formed by their planes, and the angle between the tangents is the plane angle of the dihedral angle formed by the planes of the circles.

(Art. 430.)

Therefore

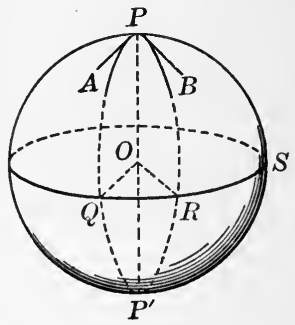

Theorem I. The angle formed by two intersecting ares of great circles has the same measure as the dihedral angle formed by the planes of the circles.

614. Suppose $P A$ and $P B$ are two given ares of great circles. Draw the great circle of which $P$ is the pole, and let it intersect $P A$ at $Q$, and $P B$ at $R$. Join $Q O$ and $R O, O$ being the centre of the sphere.

Since $P Q$ is a quadrant, $\angle P O Q$ is a right angle. Similarly $\angle P O R$ is a right angle. Therefore $\angle Q O R$ is the plane angle 
of the dihedral angle formed by the planes of $P A$ and $P B$, and is equal to the angle between the tangents at $P$, i.e. to the spherical angle $A P B$.

Now $\angle Q O R$ : four right angles $=\operatorname{arc} Q R$ : a great circle .

(Art. 267.)

Therefore $\frac{\text { spherical angle } A P B}{\text { four right spherical angles }}=\frac{\text { arc } Q R}{\text { a great circle }}$.

Since the second and fourth terms in this proportion are constant quantities, the above relation is sometimes briefly expressed by saying that "the spherical angle $A P B$ is proportional to the arc $Q R$," meaning thereby that any change in the one causes a proportional change in the other.

Therefore

THEOREM II. The angle formed by two intersecting arcs of great circles is proportional to the arc of the great circle of which the vertex of the angle is the pole, intercepted between the given arcs (produced if necessary).

615. Suppose we choose any unit of measure for angles, as for example, a degree, and take as unit of measure for arcs that arc of a great circle which subtends a unit angle at the centre of the sphere.

Thus if $A B C$ is a great circle of a sphere, and $A O B$ is a unit angle, the arc $A B$ is taken as the unit arc.

This unit arc is also frequently called a ' degree,' but care must be taken to distinguish between 'angle degree' and 'arc degree.'

Arc degrees are all equal in the same circle, or in equal circles, but are unequal in unequal

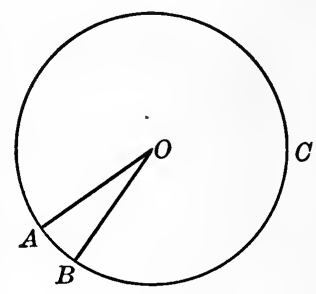
circles.

Any quadrant of a circle contains 90 arc degrees, a semicircle 180 arc degrees, and a circle 360 arc degrees.

In the diagram of Art. 613, $\angle Q O R$ will have the same measure as the arc $Q R$, the units of measure being chosen as above. But the spherical angle $Q P R$ has the same measure as $\angle Q O R$. Therefore the spherical angle $Q P R$ has the same measure as the arc $Q R$.

Hence Theorem II can be stated as follows : 
Theorem II. The angle formed by two intersecting arcs of great circles has the same measure as the arc of the great circle of which the vertex of the angle is the pole, intercepted between the given arcs (produced if necessary).

616. If, in the diagram of Article $613, Q$ is the pole of the great circle $P S P^{\prime}$, as it is the pole of some great circle through $P$ (Prop. II, Cor. III), then the spherical angle $P Q S$ has the same measure as the are $P S$.

But the arc $P S$ is a quadrant, hence $\angle P Q S$ is a right spherical angle. Now $P Q$ is any are drawn from $P$ to the great circle of which $P$ is the pole.

Therefore

Theorem III. All arcs of great circles drawn through the pole of a given great circle are perpendicular to the given great circle.

617. Definition. A spherical polygon is a closed figure on a sphere consisting of ares of great circles which intersect, two and two, in order.

The points in which the arcs intersect are the vertices of the polygon, the ares intercepted between consecutive vertices are the

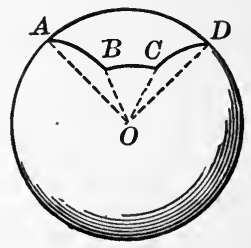
sides, and the spherical angles formed by consecutive sides are the angles of the polygon.

A spherical polygon of three sides is called a spherical triangle.

A convex spherical polygon lies wholly on one side or the other of each of its sides produced ever so far.

618. Theorem IV. No side of a convex spherical polygon can be greater than a semicircle.

For, if the side $A D$ of the spherical polygon $A B C D$ in the diagram is greater than a semicircle, $A B$ produced must intersect $A D$ between $A$ and $D$, since great circles of a sphere bisect 
each other. In that case the polygon would lie partly on one side of $A B$ and partly on the other, and hence could not be convex.

619. Definition. Two spherical polygons are identically equal when they can be made to occupy the same position, i.e. when they can be superposed.

620. The planes of the great circles, of which the sides of a spherical polygon are arcs, form at the centre of the sphere a polyhedral angle whose face angles have the same numerical measures as the sides of the polygon, and whose dihedral angles have the same measures as the angles of the polygon.

- If the spherical polygon is convex, the corresponding polyhedral angle is convex, and conversely.

Two spherical polygons are identically equal if the corresponding polyhedral angles are equal, and conversely.

Many properties of spherical polygons can be derived directly from known properties of the corresponding polyhedral angles.

\section{Proposition VII}

621. The sum of any two sides of a spherical triangle is greater than the third side.

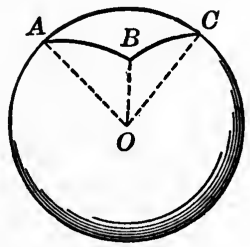

Suggestions for Proof. The sum of any two face angles of a tribedral angle is greater than the third face angle.

(Art. 459.)

How do the measures of the sides of the spherical triangle compare with the measures of the face angles of the corresponding trihedral angle? 


\section{Proposition VIII}

622. The sum of the sides of a convex spherical polygon is less than a great circle.

For the sum of the face angles of the corresponding polyhedral angle is less than four right angles.

(Art. 460.)

\section{Proposition IX}

623. If two spherical triangles lying on the same sphere, or on equal spheres, have the three sides of one equal, respectively, to the three sides of the other, their corresponding angles are also equal.

Compare Proposition XXIV, Chapter VI.

624. It should be carefully noticed that while two mutually equilateral spherical triangles are also mutually equiangular, they are not necessarily superposable.

Suppose that the two triangles $A B C$ and $A^{\prime} B^{\prime} C^{\prime}$ lie on the same sphere, and are such that the straight lines $A A^{\prime}$, $B B^{\prime}, C C^{\prime}$ are diameters of the sphere. The two trihedral angles formed at $O$ have their face angles respectively equal, and likewise their dihedral angles equal; but they are not identically equal since

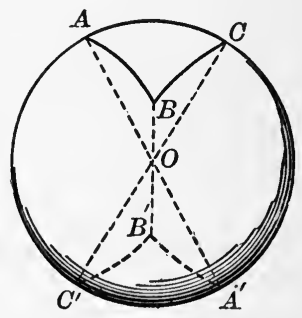
their parts are arranged in opposite orders around the vertex.

So also the triangles $A B C$ and $A^{\prime} B^{\prime} C^{\prime}$ are mutually equilateral, and likewise mutually equiangular, but their parts are arranged in opposite orders.

Such triangles cannot be superposed, but are symmetrical.

If $\triangle A^{\prime} B^{\prime} C^{\prime}$ were moved on the surface of the sphere so as to make the vertices $A^{\prime}$ and $B^{\prime}$ coincide with $A$ and $B$, respec- 
tively, the vertex $C^{\prime}$ would lie on the opposite side of $A B$ from $C$.

Two plane triangles which are mutually equilateral, and consequently mutually equiangular, could be made to coincide by turning one of them over (see Prop: IV, Chap. I), but if you should turn a spherical triangle over, it would no longer lie on the sphere.

Hence, in spherical triangles whose parts are respectively equal, it is necessary to distinguish two whose parts are arranged in the same order from two whose parts are arranged in opposite orders.

The first are identically equal, the second are symmetrical.

Proposition IX can then be stated as follows:

If two spherical triangles lying on the same sphere, or on equal spheres, have the three sides of one equal, respectively, to the three sides of the other, they are either identically equal or symmetrical, according as their parts are arranged in the same order or in opposite orders.

625. Definition. If two sides of a spherical triangle are equal, the triangle is an isosceles spherical triangle.

\section{Proposition X}

626. An isosceles spherical triangle and its symmetrical spherical triangle are identically equal.

Compare Article 463.

627. Corollary. If two sides of a spherical triangle are equal, the angles opposite those sides are equal.

Form its symmetrical triangle on the opposite side of the sphere and superpose them. $2 \mathrm{c}$ 


\section{Proposition XI}

628. If two spherical triangles lying on the same sphere, or on equal spheres, have two sides and the included angle of the one equal, respectively, to two sides and the included angle of the other, they are either identically equal or symmetrical, according as the parts are arranged in the same order or in opposite orders.

Compare Article 466.

Or, the triangles may be superposed if the parts are arranged in the same order, or one may be superposed to the symmetric of the other if the parts are arranged in opposite orders.

\section{Proposition XII}

629. If two spherical triangles lying on the same sphere, or on equal spheres, have one side and the two adjacent angles of the one equal, respectively, to one side and the two adjacent angles of the other, they are identically equal or symmetrical, according as the parts are arranged in the same order or in opposite orders.

Compare Article 467, or prove by superposition.

\section{EXERCISES}

1. If two angles of a spherical triangle are equal, the sides opposite those angles are also equal.

2. The arcs of great circles which bisect the angles of a spherical triangle are concurrent.

3. The angle between the planes of two great circles has the same measure as the arc of the great circle which joins their nearer poles.

4. If two equal circles lying in different planes have a common diameter, any plane perpendicular. to that diameter intersects the circles in four points which lie upon a circle. 


\section{SECTION III}

\section{POLAR TRIANGLES}

630. Definition. If on a sphere ares of great circles are described which have the vertices of a given spherical triangle for poles, another spherical triangle will be formed which is called the polar of the given triangle.

Let $A B C$ be any spherical triangle, and let $a^{\prime}, b^{\prime}, c^{\prime}$ be arcs of great circles whose poles are $A, B, C$. If these great circles are fully drawn, they will divide the sphere into eight spherical
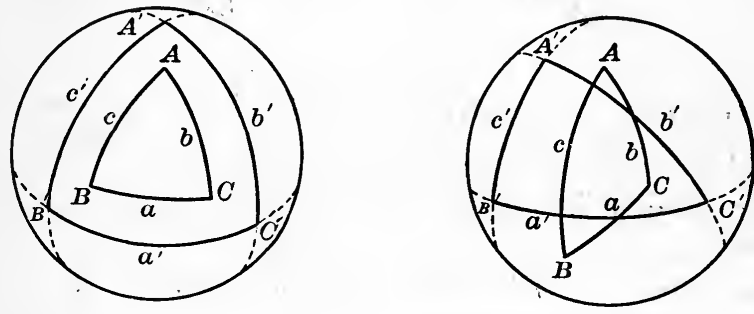

triangles. That one, $A^{\prime} B^{\prime} C^{\prime}$, of the eight is called the polar of $A B C$ which lies so that $A$ and $A^{\prime}$ are on the same side of $B C ; B$ and $B^{\prime}$, on the same side of $A C ; C$ and $C^{\prime}$, on the same side of $A B$.

That the great circles $a^{\prime}, b^{\prime}, c^{\prime}$ divide the sphere into eight triangles is easily seen if it is observed that $b^{\prime}$ and $c^{\prime}$ divide the sphere into four parts, and that $a^{\prime}$ divides each of these into two triangles.

When $A, B, C$ are used to denote the vertices of any spherical triangle, $a, b, c$ will be used to denote the opposite sides of that triangle, $A^{\prime}, B^{\prime}, C^{\prime}$ to denote the vertices of the polar triangle, $a^{\prime}, b^{\prime}, c^{\prime}$ to denote the opposite sides of the polar triangle.

Thus $A, B, C$ are the poles of the $\operatorname{arcs} a^{\prime}, b^{\prime}, c^{\prime}$, and $A^{\prime}, B^{\prime}, C^{\prime}$ are the poles of the ares $a, b, c$. 


\section{Proposition XIII}

631. If the first of two spherical triangles is the polar of the second, then the second is also the polar of the first.
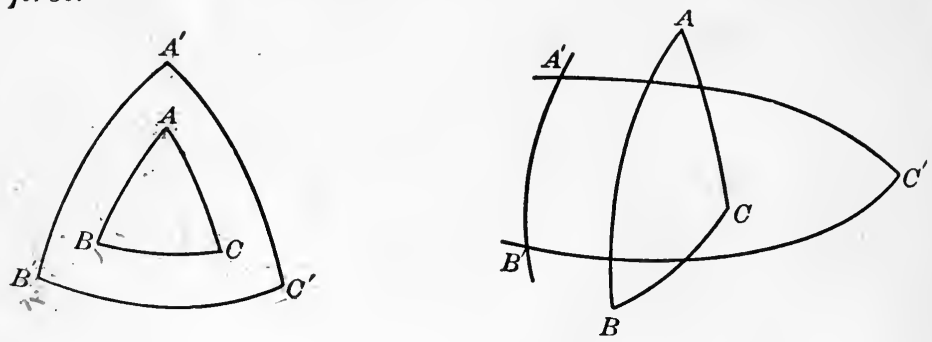

Let the two triangles $A B C$ and $A^{\prime} B^{\prime} C^{\prime \prime}$ lie on the same sphere, and suppose that $A^{\prime} B^{\prime} C^{\prime}$ is the polar triangle of $A B C$.

It is required to prove that $A B C$ is also the polar triangle of $A^{\prime} B^{\prime} C^{\prime}$.

Proof. Because $A$ is the pole of the arc $B^{\prime} C^{\prime \prime}, B^{\prime}$ is a quadrant's distance from $A$.

(Art. 596.)

And because $C$ is the pole of $A^{\prime} B^{\prime}, B^{\prime}$ is a quadrant's distance from $C$.

'Therefore $B^{\prime}$ is the pole of the arc $A C$.

(Art. 597.)

Similarly, $A^{\prime}$ is the pole of the arc $B C$, and $C^{\prime}$ is the pole of the arc $A B$.

Therefore $\triangle A B C$ is the polar triangle of $A^{\prime} B^{\prime} C^{\prime}$.

632. Definition. The supplement of any arc of a circle is that are which taken with it makes up a semicircle.

\section{EXERCISES}

1. Every point of the great circle which bisects a given arc of a great circle at right angles is equidistant from the extremities of the arc.

2. Through a given point on a sphere describe an arc of a great circle making right angles with a given arc of a great circle. 


\section{Proposition XIV}

633. In two polar triangles, the measure of any angle in one is equal to the measure of the supplement of that side in the other of which its vertex is the pole.
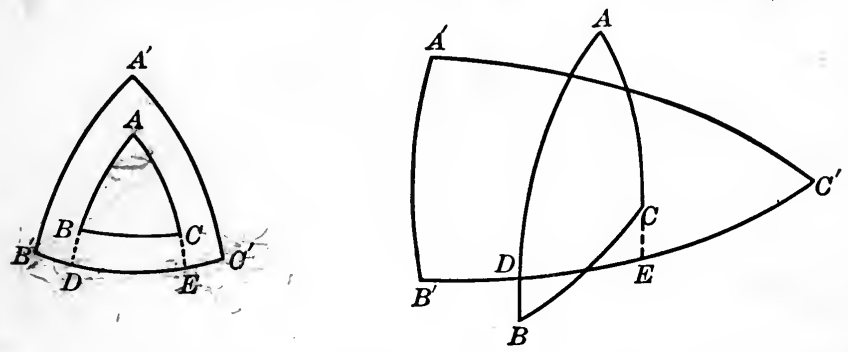

Let $A B C$ and $A^{\prime} B^{\prime} C^{\prime}$ be two polar triangles lying on any sphere, so that $A$ is the pole of $B^{\prime} C^{\prime}, B$ is the pole of $A^{\prime} C^{\prime \prime}$, and $C$ is the pole of $A^{\prime} B^{\prime}$.

It is required to prove that the measure of the angle $A$ is equal to the measure of the supplement of the arc $B^{\prime} C^{\prime}$, the measure of the angle $B$ is equal to the measure of the supplement of the are $A^{\prime} C^{\prime}$, etc.

Proof. Produce the sides $A B$ and $A C$, if necessary, to meet the side $B^{\prime} C^{\prime}$ in the points $D$ and $E$.

Then the measure of $\angle A=$ the measure of arc $D E$. (Art. 615.)

The are $B^{\prime} E$ is a quadrant. Why?

For the same reason $D C^{\prime}$ is a quadrant.

Therefore the sum of $B^{\prime} E$ and $D C^{\prime}$ is a semicircle.

But the sum of $B^{\prime} E$ and $D C^{\prime}=$ the sum of $B^{\prime} E, D E$, and $E C^{\prime}$

$$
=\text { the sum of } B^{\prime} C^{\prime} \text { and } D E \text {. }
$$

Therefore the sum of $B^{\prime} C^{\prime}$ and $D E$ is a semicircle, and the arc $D E$ is the supplement of the are $B^{\prime} C^{\prime}$.

Therefore the measure of $\angle A=$ the measure of the supplement of the arc $B^{\prime} C^{\prime}$.

Similarly the other relations can be proved. 
634. Coroldary I. If $A$ is any angle of a spherical triangle and $a^{\prime}$ the corresponding side of its polar triangle, then the measure of $a^{\prime}$ is equal to the measure of the supplement of $A$.

635. Corollary II. If any spherical triangle is equiangular, its polar triangle is equilateral ; and conversely.

636. Corollary III. If two spherical triangles on the same sphere, or on equal spheres, are mutually equiangular, their polar triangles are mutually equilateral; and conversely.

\section{Proposition XV}

637. The sum of the angles of any spherical triangle is greater than two, and less than six, right angles.

First, let $A, B, C$ be the measures of the angles of a spherical triangle, and $a^{\prime}, b^{\prime}, c^{\prime}$ the measures of the corresponding sides of its polar triangle, the units of measure in the two cases being the angle degree and the arc degree, respectively.

Then

$$
\begin{aligned}
a^{\prime} & =180-A . \\
b^{\prime} & =180-B . \\
c^{\prime} & =180-C .
\end{aligned}
$$

Therefore $\quad a^{\prime}+b^{\prime}+c^{\prime}=540-(A+B+C)$.

Now $a^{\prime}+b^{\prime}+c^{\prime}$ is less than 360 , the measure of a great circle.

(Prop. VIII.)

Therefore $540-(A+B+C)$ is less than 360 .

Hence, $A+B+C$ must be greater than 180 , which is the measure of two right angles.

That is, the sum of the angles of the triangle is greater than two right angles.

Next, $a^{\prime}+b^{\prime}+c^{\prime}$ must have some value greater than zero.

Therefore $A+B+C$ is less than 540 , which is the measure of six right angles. 


\section{Proposition XVI}

638. If two triangles on the same sphere, or on equal spheres, are mutually equiangular, they are also mutually equilateral, and are either identically equal or symmetrical, according as their parts are arranged in the same order or in opposite orders.

The proof is left to the pupil. Apply Article 636, and Proposition IX.

639. Conollary. A spherical triangle may have one, two, or three right angles, or one, two, or three obtuse angles.

\section{Definitions}

640. A spherical triangle having two right angles is called a bi-rectangular triangle, and one having three right angles is called a tri-rectangular triangle.

641. The excess of the sum of the angles of a spherical triangle over two right angles is called the spherical excess of the triangle.

\section{EXERCISES}

1. Prove that in a bi-rectangular spherical triangle the sides opposite the right angles are both quadrants.

2. Prove that if two sides of a spherical triangle are quadrants, the third side has the same measure as the opposite angle.

3. Each side of a tri-rectangular spherical triangle is a quadrant.

4. If two angles of a spherical triangle are supplementary, the sum of the sides opposite them is equal to a semicircle.

Suggestron. Produce two sides of the triangle to intersect, thus forming an adjacent triangle having two equal angles.

5. The spherical excess of a bi-rectangular spherical triangle is equal to the measure of the third angle. 


\section{Proposition XVII}

642. The shortest line that can be drawn on a sphere between two points is the arc of a great circle, not greater than a semicircle, joining the two points.

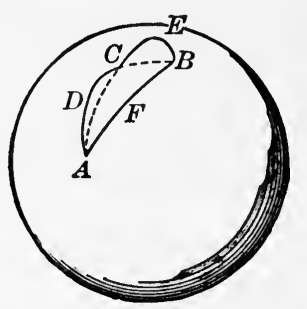

Let $A$ and $B$ be any two points on a sphere, and $A F B$ the are of a great circle, not greater than a semicircle, joining them; also let $A D C E B$ be any other path on the sphere from $A$ to $B$.

It is required to prove that the path $A F B$ is shorter than the path $A D C E B$.

Proof. In the path $A D C E B$ take any point $C$, and draw the arcs of great circles $A C$ and $C B$.

Then $A C B$ is a spherical triangle, and the sum of $A C$ and $C B$ is greater than $A B$.

(Prop. VII.)

In the path $A D C$ choose any point $D$, and draw the arcs of great circles $A D$ and $D C$.

Then $A D+D C$ is greater than $A C$.

Therefore $A D+D C+C B$ is greater than $A C+C B$, which is again greater than $A B$.

By repeating this process indefinitely the broken path of ares of great circles increases in length with each repetition, and so differs more and more from the path $A F B$.

Also the broken path of arcs of great circles can eventually be made to differ as little as we please from the given path $A D C E B$.

Therefore the path $A F B$ is shorter than the path $A D C E B$. 


\section{Proposition XVIII}

643. To find the length of the diameter of a given material sphere.
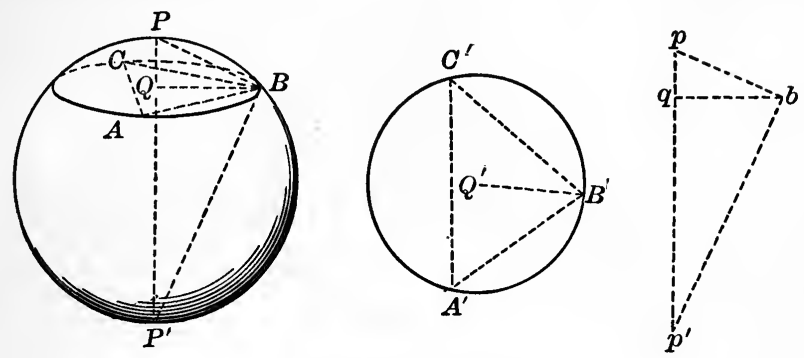

Let $P A B C$ be a given material sphere.

It is required to find the length of its diameter.

Construction. With any point $P$ on the given sphere as pole describe a circle $A B C$.

With the compasses take the lengths of the chords $A B, B C$, $C A$, and construct in any plane a triangle $A^{\prime} B^{\prime} C^{\prime}$ whose sides are equal to these three lengths. About $A^{\prime} B^{\prime} C^{\prime}$ describe a circle which will be equal to the circle $A B C$. Find its centre . $Q^{\prime}$ and the length of its radius $B^{\prime} Q^{\prime}=B Q$.

With the compasses take the length of the chord $B P$ and construct a right triangle $p b q$, having the hypotenuse $b p=B P$ and the side $b q=B Q$.

Draw $b p^{\prime}$ perpendicular to $b p$, meeting $p q$ produced at $p^{\prime}$.

Then $p p^{\prime}$ is equal in length to a diameter of the given sphere.

The proof is left to the pupil.

\section{EXERCISES}

1. Three great circles of a sphere which do not intersect in a common point, divide the surface of the sphere into four pairs of symmetrical triangles.

2. A tri-rectangular spherical triangle coincides with its polar triangle. 


\section{Section IV}

\section{AREAS OF SPHERICAL TRIANGLES}

644. Definition. The area of a spherical triangle is that portion of the sphere which is enclosed by the triangle.

645. Ахгом 15. Two spherical triangles which are identically equal have equal areas.

\section{Proposition XIX}

646. Two symmetrical spherical triangles have equal areas.

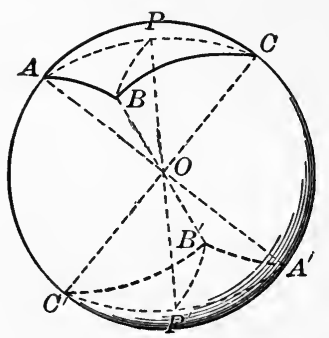

Let $A B C$ and $A^{\prime} B^{\prime} C^{\prime}$ be two symmetrical spherical triangles lying on the same sphere.

It is required to prove that $A B C$ and $A^{\prime} B^{\prime} C^{\prime}$ have equal areas.

Proof. Two symmetrical spherical triangles can always be placed so as to be vertically opposite on the sphere. Why?

Assume that the given triangles are so placed.

Let $P$ be the pole of the small circle through $A, B, C$, and draw the diameter $P O P^{\prime}$.

Draw also the arcs of great circles $P A, P B, P C, P^{\prime} A^{\prime}, P^{\prime} B^{\prime}$, $P^{\prime} C^{\prime}$.

Now also

$$
P A=P^{\prime} A^{\prime}, P B=P^{\prime} B^{\prime}, P C=P^{\prime} C^{\prime},
$$

Therefore $P^{\prime} A^{\prime}=P^{\prime} B^{\prime}=P^{\prime} C^{\prime}$. 
$\triangle \mathrm{s} P A B$ and $P^{\prime} A^{\prime} B^{\prime}$ are symmetrical triangles on the sphere, and they are also isosceles triangles.

Therefore $\triangle \mathrm{s} P A B$ and $P^{\prime} A^{\prime} B^{\prime}$ are identically equal and have equal areas.

Similarly $\triangle \mathrm{s} P B C$ and $P^{\prime} B^{\prime} C^{\prime \prime}$ have equal areas.

(Prop. X.)

Also $\triangle \mathrm{s} P A C$ and $P^{\prime} A^{\prime} C^{\prime \prime}$ have equal areas.

Therefore, adding, $\triangle \mathrm{s} A B C$ and $A^{\prime} B^{\prime} C^{\prime}$ have equal areas.

If the pole $P$ falls outside of $\triangle A B C$, instead of adding all three isosceles triangles, two should be added and the third subtracted from their sum, in order that the result may give $\triangle A B C$.

Note. The triangles mentioned in Propositions IX, XI, and XII, as being either identically equal or symmetrical, are in every case equal in area.

\section{Definitions}

647. Two great circles on a sphere divide the sphere into four parts, each of which is called a lune.

A lune then is that portion of a sphere enclosed by two halves of great circles.

The spherical angle made by the semicircles is called the angle of the lune.

Of the four lunes formed by two great circles the opposite ones have equal angles.

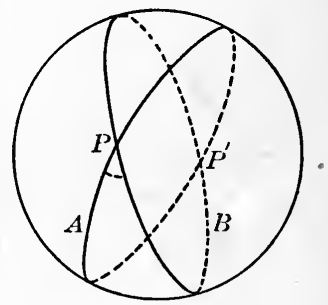

Opposite lunes on a sphere are equal, since they can be superposed.

648. Theorem. Any two lunes, having equal angles, on the same sphere or on equal spheres are identically equal.

Of two lunes having unequal angles, on the same sphere or on equal spheres, that which has the greater angle is the greater; and of two lunes having equal angles on unequal spheres, that which lies on the greater sphere is the greater. 


\section{Proposition XX}

649. The ratio of two lunes on the same sphere or on equal spheres is equal to the ratio of their angles.

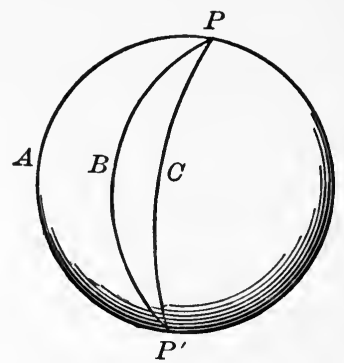

Let $P A P^{\prime} B$ and $P B P^{\prime} C$ be two lunes on the same sphere whose angles are $A P B$ and $B P C$.

It is required to prove that

$$
\text { lune } P A P^{\prime} B \text { : lune } P B P^{\prime} C=\angle A P B: \angle B P C \text {. }
$$

Outline of proof. There are two cases to be considered.

First, when $\angle \mathrm{s} A P B$ and $B P C$ are commensurable.

Take a common measure of the two angles and divide them into equal parts.

By planes through the centre of the sphere divide the lunes into parts having equal angles, and hence equal.

Next, when $\angle \mathrm{s} A P B$ and $B P C$ are incommensurable.

Take a measure of one angle and apply it as often as possible to the other. Then proceed as above, and finally make the unit of measure indefinitely small.

As a model take the proof of Prop. $\mathrm{X}$ in Chap. III.

650. Corollary. The area of a lune is to the area of the whole sphere in the same ratio as the angle of the lune is to four right angles. 


\section{Proposition XXI}

651. If two great circles intersect on a hemisphere, the areas of the two triangles formed by their arcs and arcs of the great circle bounding the hemisphere are together equal to a lune having the same angle as the angle between the great circles.

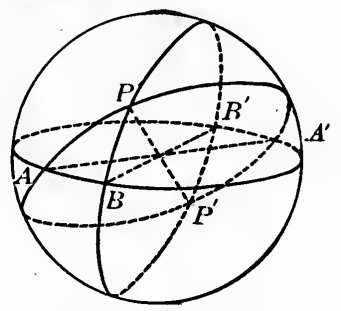

Let $A B A^{\prime} B^{\prime}$ be the great circle of the hemisphere $P A B A^{\prime} B^{\prime}$, and let the two great circles $A P A^{\prime}$ and $B P B^{\prime}$ intersect at $P$.

It is required to prove that the triangles $A P B$ and $A^{\prime} P B^{\prime}$ are together equal to the lune $P A P^{\prime} B$ whose angle is $A P B$.

Proof. $\triangle P A^{\prime} B^{\prime}$ is symmetrical with $\triangle P^{\prime} A B$.

Hence the area of $\triangle P A^{\prime} B^{\prime}=$ the area of $\triangle P^{\prime} A B$. (Prop XIX.)

But $\triangle P A B$ and $\triangle P^{\prime} A B$ make up the lune $P A P^{\prime} B$.

Therefore $\triangle P A B$ and $\triangle P A^{\prime} B^{\prime}$ are together equal in area to the lune $P A P^{\prime} B$.

\section{SPHERICAL UNITS}

652. The ordinary unit of surface is the area of a plane square whose dimensions are of unit length, but a different unit is sometimes found convenient in the measurement of spherical areas. This unit we shall eall a spherical unit of surface.

Definition. A spherical unit of surface is the area of a bi-rectangular spherical triangle whose third angle is one degree.

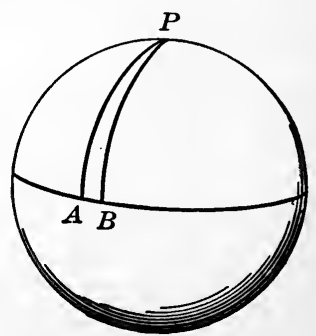


In other words, the spherical unit of surface is a half lune whose angle is one degree, the bisection being made by an arc of a great circle perpendicular to both arcs of the lune.

It should be carefully noticed that the spherical unit of surface is different on different spheres.

The area of a hemisphere is 360 spherical units; of a sphere is 720 spherical units.

The area of a bi-rectangular triangle expressed in spherical units is equal to the measure of its third angle.

The area of a lune expressed in spherical units is equal to twice the measure of its angle.

\section{Proposition XXII}

653. The area of any spherical triungle expressed in spherical units is equal to the spherical excess of the triangle.

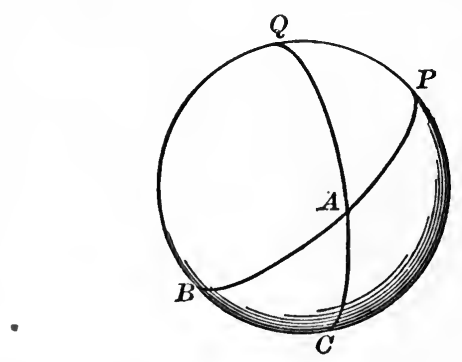

Let $A B C$ be any spherical triangle lying on the hemisphere $A B C P Q$.

It is required to prove that the area of $A B C$ expressed in spherical units equals the excess of the sum of the angles $A$, $B, C$, over two right angles.

Proof. The hemisphere consists of the four triangles $A B C$, $A C P, A P Q, A Q B$.

$\triangle A B C+\triangle A P Q=$ lune whose angle is $A$. (Prop. XXI.)

$\triangle A B C+\triangle A C P=$ lune whose angle is $B$.

$\triangle A B C+\triangle A Q B=$ lune whose angle is $C$. 
Adding the areas we get:

Twice area of $\triangle A B C+$ area of hemisphere

$=$ area of lune whose angle is $A+$ area of lune whose angle is $B$ + area of lune whose angle is $C$.

Now the area of a hemisphere equals 360 spherical units, and the area of the lune whose angle is $A$ equals $2 A$ spherical units. Therefore,

(Art. 652.)

Twice area of $\triangle A B C+360$ spherical units $=(2 A+2 B$ $+2 C)$ spherical units. Or,

Area of $\triangle A B C+180$ spherical units $=(A+B+C)$ spherical units.

Therefore area of $\triangle A B C=(A+B+C-180)$ spherical units.

But $A+B+C-180$ is the spherical excess of the triangle.

Therefore area of $\triangle A B C=$ as many spherical units as represents its spherical excess.

654. Corollary. If $S$ is the sum of the angles of a spherical polygon of $n$ sides, the area of the polygon is $[S-(n-2) 180]$ spherical units.

Divide the polygon into triangles by passing arcs of great circles through one vertex.

\section{EXERCISES}

1. If three great circles are so drawn on a sphere that each is perpendicular to the other two, into what sort of triangles do they divide the sphere? What are their areas expressed in spherical units?

2. If the sides of a spherical triangle are 75,120 , and 95 arc degrees, respectively, what is the measure of the angles of its polar triangle? What is the area of the polar triangle?

3. If the angles of a spherical triangle are 85, 135, and 65 degrees, respectively, what is its area, and what are the lengths of the sides of its polar triangle?

4. The sum of the interior angles of a convex spherical hexagon is greater than eight and less than twelve right angles. 


\section{Proposition XXIII}

655. The area of the surface generated by a line-segment revolving about an axis in its plane is equal to the length of the projection of the line-segment on the axis multiplied by the circumference of the circle whose radius is equal to that segment of the perpendicular bisector of the revolving line-segment which is intercepted between it and the axis.
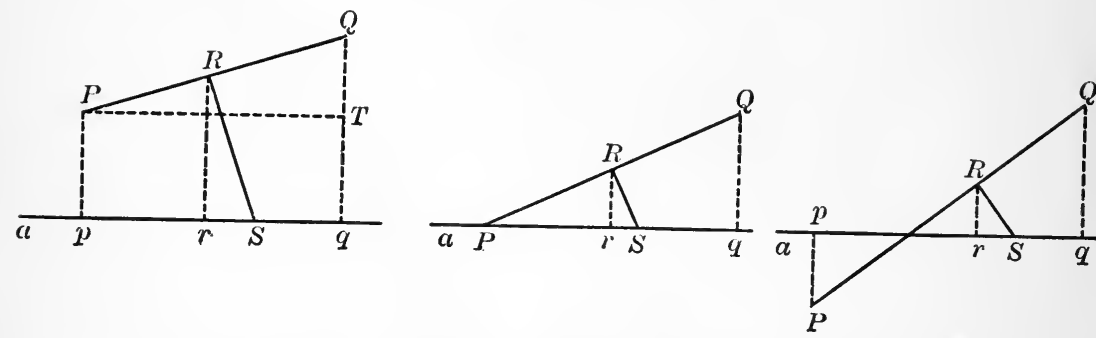

Let $P Q$ be any given line-segment which revolves about an axis $a$ lying in a plane with it; let $p q$ be the projection of $P Q$ on $a$, and $R S$ be the perpendicular bisector of $P Q$ intercepted between $P Q$ and $a$.

It is required to prove that the area of the surface generated by $P Q$ is equal to $p q$ multiplied by the circumference of the circle whose radius is $R S$.

Proof. Draw $R r$ perpendicular to $a$, and $P T$ parallel to $a$. As $P Q$ revolves about $a$ it generates the lateral surface of the frustum of a right circular cone.

The area of this surface $=P Q \times$ circ. of circle of radius $R r$.

Now $\triangle P T Q$ and $\operatorname{RrS}$ are similar. Why?

(Ex. 9, p. 367.)

Therefore, since $P T=p q$,

$$
\frac{P Q}{p q}=\frac{R S}{R r}=\frac{\text { circ. of circle of rad. } R S}{\text { circ. of circle of rad. } R r} . \quad \text { (Art. 362.) }
$$


Hence $P Q \times$ circ. of circle of rad. $R r=p q \times$ circ. of circle of rad. $R S$.

Therefore area of surface generated by $P Q=p q \times$ circ. of circle of rad. $R S$.

If $P Q$ meets $a$ at $P$, the surface generated is conical, but the proposition and proof still hold, as is easily shown.

Also, if $P Q$ is parallel to $a$ the proposition holds.

What changes are necessary in the statement of the theorem and in the proof if $P Q$ intersects $\alpha$ ?

Note. The pupil should be careful to observe that in this proposition the area spoken of is the measure of the surface expressed in ordinary plane units, so many square feet, or square inches, not in spherical units.

\section{Definitions}

656. A segment of a sphere, or a spherical segment, is the figure formed by the planes of two parallel sections of the sphere and the spherical surface intercepted between them.

The plane faces of a segment are called its bases, and the spherical surface is called a zone.

Hence, a zone is a portion of a sphere intercepted between two parallel planes which cut the sphere.

If one of the parallel planes forming the bases of a spherical segment is tangent to the sphere, the segment is a segment of one base, and its spherical surface is a zone of one base.

The perpendicular distance between the bases of a spherical segment is the altitude of the segment. It is also the altitude of the zone.

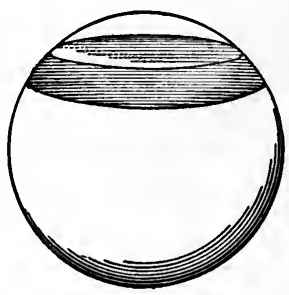

Spherical segment

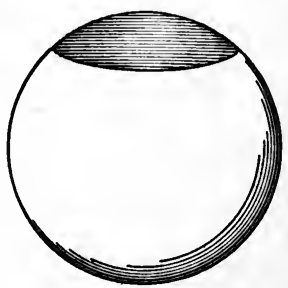

segment of base 


\section{Proposition XXIV}

657. The area of a zone is equal to the product of its altitude and the circumference of a great circle.

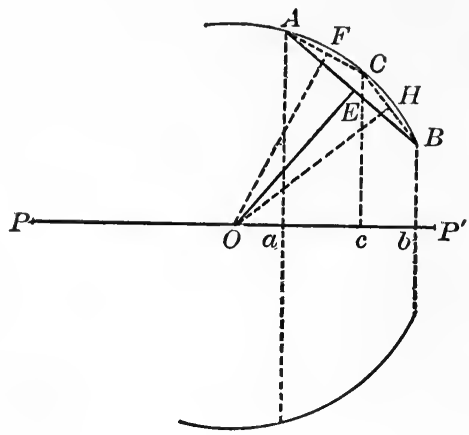

Let $A B$ be an are of a great circle of a sphere whose centre is $O$ and diameter $P P^{\prime}$; and suppose $A B$ to rotate about the diameter $P P^{\prime}$ so as to generate a zone whose altitude is $a b$.

It is required to prove that the area of the zone generated by $A B$ is equal to the product of $a b$ and the circumference of a great circle of the sphere.

Proof. Draw the chord $A B$ and the perpendicular bisector $E O$, which passes through the centre of the sphere.

The area of the surface generated by the chord $A B$ as it rotates about $P P^{\prime}=a b \times$ circ. of a circle of rad. $O E$.

(Prop. XXIII.)

Let the arc $A B$ be bisected at $C$, and draw the chords $A C$ and $C B$. Also the perpendicular bisectors $O F$ and $O H$.

Let $c$ be the projection of $C$ upon $P P^{\prime}$.

Then the area of the surface generated by the two chords $A C$ and $C B$ as they rotate about $P P^{\prime}=a c \times$ circ. of circle of rad. $O F+c b \times$ circ. of circle of rad. $O H$

$=a b \times$ circ. of circle of rad. $O F$, since $O H=O F$. Why? 
If this process of bisecting the arcs is repeated indefinitely, the series of chords approaches the arc $A B$ as a limit, and the perpendicular bisector approaches a radius $r$ of the sphere as a limit.

Therefore the area of the surface generated by the arc $A B$ as it rotates about $P P^{\prime}=a b \times$ circ. of circle of rad. $r$.

That is, the area of a zone whose altitude is $a b=a b \times$ circ. of a great circle.

658. Corollary I. The area of a zone whose altitude is $h$, lying on a sphere of radius $r$, equals $2 \pi r h$.

659. Definition. A right circular cylinder the radius of whose base equals the radius of a sphere, and whose altitude equals the diameter of the sphere, when placed so as to enclose the sphere, is called the enveloping cylinder of the sphere.

660. Corollary II. The area of a zone is equal to that portion of the lateral area of the enveloping cylinder which is intercepted between the same planes as the zone.

For the circumference of the base of the enveloping cylinder is $2 \pi r$, and if $h$ is the distance between the planes of section, the lateral area of the cylinder intercepted between them is $2 \pi r h$ (Art. 561) and this is also the area of the intercepted zone.

\section{EXERCISES}

1. Find the area of a zone of altitude 3 inches on a sphere whose radius is 10 inches.

2. The diameter of a given sphere is 10 inches and it is desired to cut the surface by parallel planes, into five parts of equal area. Locate the planes of section.

3. A slice is cut from a sphere of radius 5 inches by a plane passing 3 inches from the centre; what is the area of the spherical surface, and also of the plane surface of the slice?

4. Prove that the area of a zone of one base is equal to the area of a circle whose radius is the chord of the generating arc of the zone. 


\section{Proposition XXV}

661. The area of a sphere is equal to the product of a diameter and the circumference of a great circle.

This follows directly from Proposition XXIV, the generating arc being a semicircle.

662. Conollary I. The area of a sphere whose radius is $r$ is given by the formula:

$$
A=2 r \times 2 \pi r=4 \pi r^{2} .
$$

663. Corollary II. The areas of two spheres are in the same ratio as the squares of their radii, or as the squares of their diameters.

664. Cokollary III. The area of a sphere equals four times the area of one of its great circles.

665. Conollaky IV. The area of a sphere equals the lateral area of its enveloping cylinder.

666. Conollary V. The area of a spherical unit expressed in plane units equals $\frac{\pi r^{2}}{180}$.

Nоте. All the above areas are expressed in plane units.

\section{EXERCISES}

1. What is the area of a sphere whose radius is 10 inches ?

2. A sphere has a radius of 9 inches. What is the radius of a sphere having double the area?

3. Two parallel planes cut a sphere of radius 10 inches, 6 and 8 inches, respectively, from the centre. Find the area of the zone so formed (1) when the planes are on the same side of the centre, (2) when the planes are on opposite sides of the centre.

4. A triangle whose angles are $80^{\circ}, 120^{\circ}, 135^{\circ}$ lies on a sphere of radius 16 inches. Find its area.

SugGestion. First find the area in spherical units and then reduce to plane units. 


\section{Section V}

\section{VOLUME OF THE SPHERE}

\section{Proposition XXVI}

667. The volume of a sphere is equal to one-third the product of its area and its radius.

Suppose that a polyhedron of any number of sides has been circumscribed about a sphere. Through all the edges of the polyhedron and the centre of the sphere pass planes.

This divides the polyhedron into as many pyramids as the polyhedron has faces. - The altitude of each pyramid is equal to a radius of the sphere, since each face of the polyhedron is tangent to the sphere.

The volume of any one of the pyramids $=\frac{1}{3} r \times$ area of its base (Art. 523), and the sum of all the bases of the pyramids equals the surface of the polyhedron.

Therefore, volume of the polyhedron $=\frac{1}{3} r \times$ the surface area of the polyhedron, and this relation holds no matter how many faces the polyhedron may have.

Now by increasing the number of faces of the circumscribed polyhedron indefinitely, it can be made to approach the sphere as its limit, and its volume will approach the volume of the sphere as its limit.

Therefore, volume of a sphere $=\frac{1}{3} r \times$ area of the sphere.

668. Corollary I. The volume of a sphere can be expressed by the following formula:

$$
V=\frac{1}{3} r \times 4 \pi r^{2}=\frac{4}{3} \pi r^{3} .
$$

669. Corollary II. The volumes of two spheres are in the same ratio as the cubes of their radii, or as the cubes of their diameters.

\section{EXERCISES}

1. Show that the volume of a sphere is equal to two-thirds of the volume of the enveloping cylinder. 


\section{Definitions}

670. A spherical pyramid is a figure consisting of a spherical polygon for base and plane faces through the sides of the polygon.

The lateral edges of the pyramid are all radii of the sphere.

671. A spherical sector is a figure consisting of a zone and two conical surfaces, having the centre of the sphere for vertex, and passing through the circular boundaries of the zone.

If the zone is a zone of one base, then the spherical sector has but one conical surface.

The zone is called the base of the spherical sector.

If a sector of a circle is rotated about any diameter of the circle, it will generate a spherical sector. If it is rotated about one of its own radii, the base of

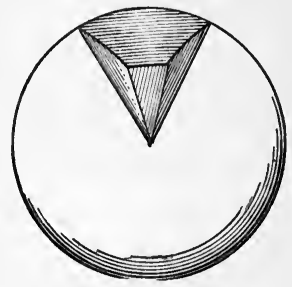

Spherical pyramid

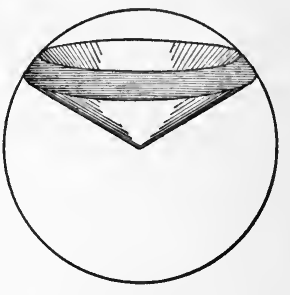

Spherical sector the spherical sector will be a zone of one base.

672. Theorem. The volume of a spherical pyramid or a spherical sector is to the volume of its sphere in the same ratio as the area of its base is to the area of the sphere.

673. If $V$ represents the volume of a spherical pyramid or sector, $V^{\prime}$ the volume of the sphere, $A$ the area of the base of the pyramid or sector, and $A^{\prime}$ the area of the sphere,

then

$$
\frac{V}{V^{\prime}}=\frac{A}{A^{\prime}},
$$

whence

$$
V=A \cdot \frac{V^{\prime}}{A^{\prime}}=A \cdot \frac{\frac{4}{3} \pi r^{3}}{4 \pi r^{2}}=\frac{1}{3} A r .
$$

That is to say :

The volume of a spherical pyramid, or of a spherical sector, is equal to one-third the product of the area of its base and the radius of the sphere. 


\section{Proposition XXVII}

674. To find the volume of a spherical segment.
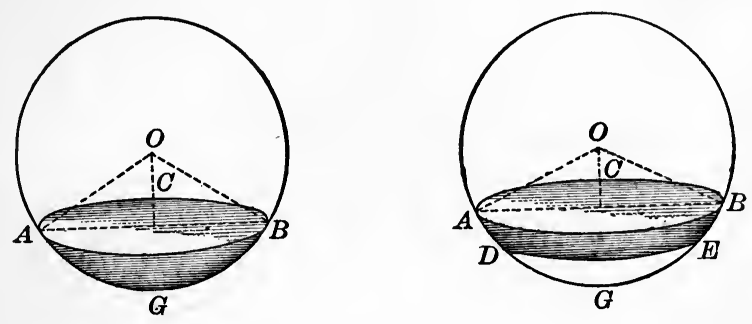

First, to find the volume of a spherical segment of one base.

Suggestions. Find the area of the zone $G-A B C$. (Art. 657.) Find the volume of the spherical sector $O-A B C G$. (Art. 673.) Subtract the volume of the cone $O-A B C$.

Hence volume of the segment $G-A B C$

$$
=\frac{2}{3} \pi r^{2} h-\frac{1}{3} \pi a^{2}(r-h),
$$

where $r=$ radius of the sphere, $a=$ radius of base of the segment, $h=$ altitude of the segment.

Then

$$
V=\frac{\pi}{3}\left[2 r^{2} h-a^{2} r+a^{2} h\right] .
$$

Now

$$
h(2 r-h)=a^{2} \text {. }
$$

Hence

$$
2 r^{2} h=a^{2} r+h^{2} r \text {. }
$$

Therefore

$$
\begin{aligned}
V & =\frac{\pi}{3}\left[h^{2} r+a^{2} h\right] \\
& =\frac{1}{2} \pi a^{2} h+\frac{1}{6} \pi h^{3}, \text { since } r=\frac{a^{2}+h^{2}}{2 h} .
\end{aligned}
$$

Next, to find the volume of a spherical segment of two bases lying on the same side of the centre of the sphere.

Let $a$ and $b$ be the radii of the two bases, $a$ being the greater, and $h_{1}$ the altitude of a segment of one base of radius $a, h_{2}$ the 
altitude of a segment of one base of radius $b$, so that $h_{1}-h_{2}=h$, the altitude of the given segment.

Then the volume of the given segment

$$
\begin{aligned}
& =\left[\frac{1}{2} h_{1} \cdot \pi a^{2}+\frac{1}{6} \pi h_{1}{ }^{3}\right]-\left[\frac{1}{2} h_{2} \cdot \pi b^{2}+\frac{1}{6} \pi h_{2}{ }^{3}\right] \\
& =\frac{\pi}{2}\left[h_{1} a^{2}-h_{2} b^{2}\right]+\frac{\pi}{6}\left[h_{1}^{3}-h_{2}{ }^{3}\right] \\
& =\frac{\pi}{2}\left[h_{1} a^{2}+h_{1} b^{2}-h_{2} a^{2}-h_{2} b^{2}\right]+\frac{\pi}{6}\left[h_{1}{ }^{3}-h_{2}{ }^{3}+3 h_{2} a^{2}-3 h_{1} b^{2}\right]
\end{aligned}
$$

having added and subtracted $h_{1} b^{2}$ and $h_{2} a^{2}$.

Now

$$
\begin{aligned}
& h_{1}\left(2 r-h_{1}\right)=a^{2}, \text { or } 2 r h_{1}-h_{1}{ }^{2}=a^{2} ; \\
& h_{2}\left(2 r-h_{2}\right)=b^{2}, \text { or } 2 r h_{2}-h_{2}{ }^{2}=b^{2} .
\end{aligned}
$$

Multiplying the first line by $3 h_{2}$, the second by $3 h_{1}$, and subtracting, gives

Therefore

$$
3 h_{2} a^{2}-3 h_{1} b^{2}=3 h_{1} h_{2}^{2}-3 h_{1}{ }^{2} h_{2} .
$$

$$
\begin{aligned}
V & =\frac{\pi}{2}\left[h_{1} a^{2}+h_{1} b^{2}-h_{2} a^{2}-h_{2} b^{2}\right]+\frac{\pi}{6}\left[h_{1}^{3}-h_{2}^{3}+3 h_{1} h_{2}^{2}-3 h_{1}^{2} h_{2}\right] \\
& =\frac{\pi}{2}\left[\left(a^{2}+b^{2}\right)\left(h_{1}-h_{2}\right)\right]+\frac{\pi}{6}\left[\left(h_{1}-h_{2}\right)^{3}\right] \\
& =\frac{\pi}{2}\left(a^{2}+b^{2}\right) h+\frac{\pi}{6} h^{3} \\
& =\frac{1}{2} h\left(\pi a^{2}+\pi b^{2}\right)+\frac{1}{6} \pi h^{3} .
\end{aligned}
$$

This result may be stated in words as follows:

The volume of a spherical segment is equal to the product of its altitude and half the sum of the areas of its bases together with the volume of a sphere whose diameter is equal to the altitude of the segment.

The algebraic difficulties in the reduction are only introduced for the sake of giving the result in a convenient form. It should be noticed that the last formula for the volume will reduce to that given for a segment of one base by putting $b=0$. 


\section{MISCELLANEOUS EXERCISES}

Note. Use $3 \frac{1}{7}$ as the approximate value of $\pi$.

1. Find the area and the volume of a sphere whose radius is 10 inches.

2. How many square inches in the spherical unit on a sphere whose diameter is 14 inches?

3. Find in square inches the area of a spherical triangle the sum of whose angles is 210 degrees, on a sphere whose radius is 15 inches.

4. On a sphere of radius 12 inches, there is described a spherical triangle and a spherical quadrilateral, the sum of each of whose angles is 450 degrees. What is the difference in their areas?

5. Find the area of a zone of height 3 inches on a sphere of radius 10 inches. Does it matter where the zone is placed on the sphere?

6. The radii of two spheres are in the ratio of $2: 3$. Compare their areas and their volumes.

7. The area of a sphere is 1000 square inches; what is the area of one of its great circles?

8. Two spheres of lead, of radius 2 inches and 3 inches, respectively, are melted into a single sphere. Find its radius.

9. A sphere is such that its number of units of volume equals twice its number of units of area. Find its radius.

10. The base of a spherical pyramid is a pentagon the sum of whose angles is 900 degrees. Find the volume of the pyramid if the radius of the sphere is 12 inches.

11. If the area of a zone of one base is one-quarter of the area of the aphere, show that the altitude of the zone is half the radius of the sphere.

12. How far must the eye be from a sphere of radius 16 inches in order to see one-quarter of it?

13. If the radius of the base of a circular cone and of a circular cylinder equals the radius of a sphere and they are all of the same height, show that the volumes of the cone and sphere are together equal to the volume of the cylinder.

14. The radii of the bases of a spherical segment are 6 and 8 inches, respectively, and its altitude is 2 inches. Find its volume, the radius of the sphere from which it was cut, the areas of its bases, and its lateral area.

15. Find the volume of a sphere whose radius is $\mathbf{1 4}$ feet.

16. The volume of a sphere is 10286 cubic inches. Find its area. 
1. Definitions.

\section{SUMMARY OF CHAPTER IX}

(1) Sphere - a closed surface such that all points of it are equidistant from a fixed point within it. $\S 580$.

(2) Centre, Radius, Diameter. $\$ 580$.

(3) Concentric Spheres - two spheres having the same centre, but not coinciding. $\$ 582$.

(4) Great Circle of a Sphere - a circle lying on the sphere, whose plane passes through the centre of the sphere. $§ 585$.

(5) Quadrant-one-quarter of a great circle. $\$ 585$.

(6) Axis of a Circle of a Sphere - the diameter of the sphere perpendicular to the plane of the circle. $§ 592$.

(7) Poles of a Circle of a Sphere - the extremities of the axis of the circle. $\$ 592$.

(8) Tangent to a Sphere-a line or plane which meets the sphere at one, and only one, point. $\S 600$.

(9) Inscribed Polyhedron-one whose vertices lie on the sphere $\S 606$.

(10) Circumscribed Polyhedron - one whose faces are tangent to the sphere. $\$ 606$.

(11) Spherical Angle - the angle formed by two intersecting arcs of great circles, i.e. by the tangents at their common point. $§ 612$.

(12) Spherical Polygon-a closed figure on a sphere consisting of arcs of great circles which intersect, two and two, in order. $\S 617$.

(13) Spherical Excess of a Triangle - the excess of the sum of the angles of a spherical triangle over two right angles. $\$ 641$.

(14) Area of a Spherical Triangle - that portion of the sphere which is enclosed by the triangle, or the measure of that portion. $\S 644$.

(15) Lune-a portion of a sphere enclosed by two halves of great circles. $\S 647$.

(16) Angle of a Lune - the angle between its boundaries. $\$ 647$.

(17) Spherical Unit of Surface - the area of a bi-rectangular spherical triangle whose third angle is one degree. $\$ 652$.

(18) Spherical Segment - the figure formed by the planes of two parallel sections of a sphere and the spherical surface intercepted between them. $\S 656$.

(19) Zone - the portion of a sphere intercepted between two parallel planes which cut the sphere. $\S 656$.

(20) Altitude of a Spherical Segment or Zone-the distance between its bases. $\S 656$. 
(21) Spherical Pyramid-a figure consisting of a spherical polygon for base, and plane faces through the sides of the polygon. $\S 670$.

(22) Spherical Sector - a figure consisting of a zone and two conical surfaces through the circular boundaries of the zone, having the centre of the sphere for vertex. $\$ 671$.

(23) Enveloping cylinder of a sphere - a right circular cylinder the radius of whose base equals the radius of the sphere, and whose altitude equals the diameter of the sphere, placed so as to enclose the sphere. $\S 659$.

2. A хуомs.

(1) Two spherical triangles which are identically equal have equal areas (Axiom 15). $§ 645$.

3. Problems.

(1) To find the length of the diameter of a given material sphere. $\S 643$.

4. Theorems on the Properties of a Sphere and its Plane Sections.

(1) Equal spheres have equal radii and equal diameters, and conversely. $\S 581$.

(2) The section of a sphere made by a plane is a circle. $\$ 583$.

(3) Sections of a sphere made by planes equidistant from the centre are equal circles, and of two sections made by planes unequally distant from the centre, that is the greater circle which is made by the nearer plane. $\S 584$.

(4) The centre of any circle of a sphere is the foot of the perpendicular drawn from the centre of the sp'sere to the plane of the circle. $\$ 586$.

(5) The centres of all great circles coincide with the centre of the sphere, and all great circles on the same sphere are equal. $\S 587$.

(6) Every great circle divides the sphere into two equal parts. $§ 588$.

(7) Any two great circles on the same sphere bisect each other. $\S 589$.

(8) An arc of a great circle can be drawn through any two given points on a sphere, and if the two given points are not the extremities of a diameter, only one such arc less than a semicircle can be drawn. $\S 590$. 
(9) Through three given points on a sphere, one and only one circle can be drawn. $\$ 591$.

(10) All points of any circle of a sphere are equidistant from either of its poles. $\$ 594$.

(11) The polar distance of any point of a great circle is a quadrant. $\S 596$.

(12) If a point on a sphere is a quadrant's distance from each of two given points on the sphere, it is the pole of the great circle passing through these two points. $\$ 597$.

(13) If one point on a sphere is a quadrant's distance from another, it is the pole of some great circle passing through the other. $\S 598$.

(14) The intersection of two spheres is a circle, the plane of which is perpendicular to the straight line joining the centres of the spheres, and the centre of which is on that line. $\S 604$.

\section{Theorems relating to Tangents to a Sphere.}

(1) A plane which is perpendicular to a radius of a sphere at its extremity is tangent to the sphere, and conversely. $\$ 601$.

(2) All lines tangent to a sphere at one point lie in a plane tangent to the sphere at that point. $\$ 602$.

(3) The plane of two straight lines tangent to a sphere at the same point is also tangent to the sphere at that point. $\S 603$.

6. Theorems relating to the Properties of Spherical Angles, Triangles, and Polygons.

(1) The angle formed by two intersecting arcs of great circles has the same measure as the dihedral angle formed by the planes of the circles. $\$ 613$.

(2) The angle formed by two intersecting arcs of great circles has the same measure as the arc of the great circle of which the vertex of the angle is the pole, intercepted between the given arcs (produced if necessary). $\$ 615$.

(3) All arcs of great circles drawn through the pole of a given great circle are perpendicular to the given great circle. $§ 616$.

(4) No side of a convex spherical polygon can be greater than a semicircle. $\$ 618$.

(5) The sum of any two sides of a spherical triangle is greater than the third side. $\$ 621$.

(6) The sum of the sides of a convex spherical polygon is less than a great circle. $\$ 622$. 
(7) An isosceles spherical triangle and its symmetrical spherical triangle are identically equal. $\$ 626$.

(8) If two sides of a spherical triangle are equal, the angles opposite those sides are equal. $\$ 627$.

(9) If the first of two spherical triangles is the polar of the second, then the second is also the polar of the first. $\$ 631$.

(10) In two polar triangles, the measure of any angle in one is equal to the measure of the supplement of that side in the other of which its vertex is the pole. $\S 633$.

(11) If $A$ is any angle of a spherical triangle, and $a^{\prime}$ the corresponding side of the polar triangle, then the measure of $a^{\prime}$ is equal to the measure of the supplement of $A . \quad \S 634$.

(12) If any spherical triangle is equiangular, its polar triangle is equilateral, and conversely. $\S 635$.

(13) If two spherical triangles on the same sphere, or on equal spheres, are mutually equiangular, their polar triangles are mutually equilateral, and conversely. $\S 636$.

(14) The sum of the angles of any spherical triangle is greater than two right angles and less than six right angles. $\$ 637$.

(15) A spherical triangle may have one, two, or three right angles, or one, two, or three obtuse angles. $§ 639$.

\section{Theorems on the Equality of Spherical Triangles.}

Two spherical triangles lying on the same or equal spheres are identically equal, or symmetrical, if they have -

(1) Three sides of the one equal, respectively, to the three sides of the other. $\S 623$.

(2) Two sides and the included angle of the one equal, respectively, to two sides and the included angle of the other. $\$ 628$.

(3) One side and the two adjacent angles of the one equal, respectively, to one side and the two adjacent angles of the other. $\S 629$.

(4) Three angles of the one equal, respectively, to the three angles of the other. $\S 638$.

8. Theorems relativg to Areas.

(1) Two symmetrical spherical triangles have equal areas. $\$ 646$.

(2) Any two lunes on the same sphere or on equal spheres, having equal angles, are equal. $\$ 648$.

(3) The ratio of two lunes on the same sphere or on equal spheres is equal to the ratio of their angles. $\S 649$. 
(4) The area of a lune is to the area of the whole sphere in the same ratio as the angle of the lune is to four right angles. $\$ 650$.

(5) If two great circles intersect on a hemisphere, the two triangles formed by their arcs and arcs of the great circle bounding the hemisphere are together equal to a lune having the same angle as the angle between the great circles. $\$ 651$.

(6) The area of any spherical triangle expressed in spherical units is equal to the spherical excess of the triangle. Area $=A+B+$ $C-180$. $\$ 653$.

(7) If $S$ is the sum of the angles of a spherical polygon of $n$ sides, the area of the polygon is $[S-(n-2) 180]$ spherical units. $\S 654$.

(8) The area of the surface generated by a line-segment revolving about an axis in its plane is equal to the length of the projection of the line-segment on the axis multiplied by the circumference of the circle whose radius is equal to that segment of the perpendicular bisector of the revolving line-segment which is intercepted between it and the axis. $\S 655$.

(9) The area of a zone is equal to the product of its altitude and the circumference of a great circle. $A=2 \pi r h . \quad \S 657$.

(10) The area of a zone is equal to that portion of the lateral area of the enveloping cylinder which is intercepted between the same planes as the zone. $\$ 660$.

(11) The area of a sphere is equal to the product of a diameter and the circumference of a great circle. $A=2 \pi r d=4 \pi r^{2} . \quad \S 661$.

(12) The areas of two spheres are in the same ratio as the squares of their radii, or as the squares of their diameters. $A: A^{\prime}=r^{2}: r^{\prime 2}$, or $d^{2}: d^{\prime 2}$. $§ 663$.

(13) The area of a sphere equals four times the area of one of its great circles. $\$ 664$.

(14) The area of a sphere equals the lateral area of its enveloping cylinder. $\S 665$.

(15) The area of a spherical unit expressed in plane units equals $\frac{\pi r^{2}}{180} \cdot \S 666$.

9. Theorems relating to Volumes.

(1) The volume of a sphere is equal to one-third the product of its area and its radius. $\quad V=\frac{4}{3} \pi r^{3} . \quad \S 667$.

(2) The volumes of two spheres are in the same ratio as the cubes of their radii, or as the cubes of their diameters. $V: V^{\prime}=r^{3}: r^{\prime 3}$, or $d^{3}: d^{\prime 3}$. $\$ 669$. 
(3) The volume of a spherical pyramid or a spherical sector is equal to one-third the product of the area of its base and the radius of the sphere. $\quad V=\frac{1}{3} A r . \quad \S 673$.

(4) The volume of a spherical segment is equal to $\frac{1}{2} h\left(\pi a^{2}+\pi b^{2}\right)$ $+\frac{1}{6} \pi h^{3}$, where $a$ and $b$ are the radii of its bases and $h$ its altitude. $\$ 674$.

10. Miscellaneous Theorems.

(1) Through any four points not lying in the same plane, one and only one sphere can be passed. $\$ 605$.

(2) The perpendiculars to the four faces of a tetrahedron, erected at the centres of the circumscribed circles, all pass through one point. $\S 608$.

(3) The six planes perpendicular to the edges of a tetrahedron at their mid-points have one point in common. $\$ 609$.

(4) One and only one sphere can be inscribed in any given tetrahedron. $\$ 610$.

(5) The planes which bisect the six dihedral angles of any tetrahedron have one point in common. $\$ 611$.

(6) The shortest line that can be drawn on a sphere between two points is the arc of a great circle, not greater than a semicircle, joining the two points. $\$ 642$. 


\section{APPENDIX}

The following brief introduction to Trigonometry is designed to give to the high school or academy pupil as much of that subject as he may need for a course in Physics or Elementary Mechanics. It contains no solid geometry, and may be read as soon as the pupil has completed Chapter $\mathrm{V}$ of this text, or earlier if desired.

\section{Trigonometric Ratios}

Let $B A C$ be any angle (for convenience at present, an acute angle), and from a point $P$ in one boundary let a perpendicular $P M$ be drawn to the other.

Then considering the lengths of the line-segments $A P, A M, M P$,

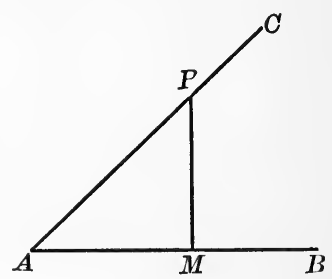

FIG. 1.

the ratio $\frac{M P}{A P}$ is called the sine of the angle $A$, the ratio $\frac{A M}{A P}$ is called the cosine of the angle $A$, the ratio $\frac{M P}{A M}$ is called the tangent of the angle $A$.

If the point $P$ were chosen differently on the boundary $A C$, and the perpendicular were drawn to $A B$, would the ratios of the sides of this new triangle be equal to the corresponding ratios of the sides of the triangle PAM?

Or, if the point $P$ were chosen in the boundary $A B$ and the perpendicular drawn to $A C$, would the ratios of the sides be altered ? 
If the size of the angle $A$ were changed, would the values of these ratios be changed?

The answers to these questions will show that the values of the sine, cosine, and tangent of the angle $A$ do not depend upon the position of the point $P$, but do depend upon the size of the angle.

From these considerations it follows that equal angles have equal sines, cosines, and tangents; unequal angles have unequal sines, cosines, and tangents.

In the right triangle $P A M$, the side $A P$ is the hypotenuse; $M P$, the side opposite the vertex $A$, may be called the perpendicular; and $A M$, the side adjacent to $A$, may be called the base.

Then sine of $\angle A=\frac{M P}{A P}=\frac{\text { side opposite }}{\text { hypotenuse }}$ or $\frac{\text { perpendicular }}{\text { hypotenuse }}$,

cosine of $\angle A=\frac{A M}{A P}=\frac{\text { side adjacent }}{\text { hypotenuse }}$ or $\frac{\text { base }}{\text { hypotenuse }}$, tangent of $\angle A=\frac{M P}{A M}=\frac{\text { side opposite }}{\text { side adjacent }}$ or $\frac{\text { perpendicular. }}{\text { base }}$.

The reciprocals of these ratios have also received particular names as follows:

$$
\begin{gathered}
\frac{1}{\text { sine of } \angle A}=\text { cosecant of } \angle A=\frac{A P}{M P}=\frac{\text { hypotenuse }}{\text { side opposite }}, \\
\frac{1}{\text { cosine of } \angle A}=\text { secant of } \angle A=\frac{A P}{A M}=\frac{\text { hypotenuse }}{\text { side adjacent }}, \\
\frac{1}{\text { tangent of } \angle A}=\text { cotangent of } \angle A=\frac{A M}{M P}=\frac{\text { side adjacent }}{\text { side opposite }}
\end{gathered}
$$

For brevity, we write ' $\sin A$ ' instead of ' sine of $\angle A$ ' but this symbol should always be understood to mean 'the sinc of the angle $A$.'

Similarly we write $\cos A, \tan A, \cot A, \sec A, \operatorname{cosec} A$, instead of ' cosine of $\angle A$,' ' tangent of $\angle A$,' etc.

It should be noticed carefully that $\sin A, \cos A$, etc., are mere numbers, being ratios between the lengths of certain linesegments. 
To find the trigonometric ratios of $\angle A$ (Fig. 1), $P M$ was drawn from a point in one boundary perpendicular to the other. If we wish to find the ratios of $\angle P$, we can look upon $A M$ as drawn from a point $A$ in one boundary of $P$, perpendicular to the other boundary, so that $\triangle P A M$, which is right-angled at $M$, will give us the ratios of both $\angle A$ and $\angle P$.

Thus

$$
\begin{aligned}
& \sin A=\frac{M P}{A P}=\cos P, \\
& \cos A=\frac{A M}{A P}=\sin P, \\
& \tan A=\frac{M P}{A M}=\cot P .
\end{aligned}
$$

That is to say,

the sine of an angle = the cosine of its complement,

the cosine of an angle = the sine of its complement,

the tangent of an angle $=$ the cotangent of its complement.

2. Relations Among the Ratios

In the right triangle $A B C, C$ being the right angle,

$$
\overline{B C}^{2}+\overline{A C}^{2}=\overline{A B}^{2},
$$

no matter how large or how small the angles $A$ and $B$ may be.

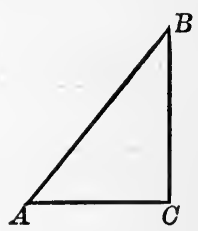

Divide this relation through by $\overline{A B}^{2}$ and you obtain

$$
\frac{\overline{B C}^{2}}{\overline{A B}^{2}}+\frac{\overline{A C}^{2}}{\overline{A B}^{2}}=\frac{\overline{A B}^{2}}{\overline{A B}^{2}}
$$

or

$$
\left(\frac{B C}{A B}\right)^{2}+\left(\frac{A C}{A B}\right)^{2}=1
$$

Now

$$
\frac{B C}{A B}=\sin A, \frac{A C}{A B}=\cos A .
$$

Hence

$$
(\sin A)^{2}+(\cos A)^{2}=1 \text {. }
$$


This relation is usually written

$$
\sin ^{2} A+\cos ^{2} A=1,
$$

and should be read "sine-squared $A$ plus cosine-squared $A$ equals one."

Also, in the right triangle $A B C$,

$$
\begin{gathered}
\frac{\sin A}{\cos A}=\frac{B C}{A B} \div \frac{A C}{A B}=\frac{B C}{A C}=\tan A, \\
\text { i.e. } \frac{\sin A}{\cos A}=\tan A .
\end{gathered}
$$

\section{EXERCISES}

1. Is $\sin A$ greater or less than unity? $\cos A$ ? $\sec A$ ? $\operatorname{cosec} A$ ?

2. Suppose you keep the point $P$ fixed on the line $A C$ (Fig. 1), while the line rotates so as to make the angle $A$ increase. Will $\sin A$ increase or decrease? $\cos A$ ? $\tan A$ ?

3. Can $\tan A$ ever be greater than unity? Can it be less than unity? For what value of $A$ will it be equal to unity ?

4. $A B C$ is a triangle right-angled at $C, A$ being the least angle. If the lengths of the sides are $3 \mathrm{ft}$., $4 \mathrm{ft}$., and $5 \mathrm{ft}$. respectively, find the values of $\sin A, \cos A, \tan A, \cos B, \tan B, \sin B$.

5. Construct an angle whose sine is $\frac{1}{2}$.

Suggestion. Construct a right triangle whose hypotenuse is equal to twice one side.

6. Construct an angle whose cosine is $\frac{2}{3}$.

7. Construct an angle whose tangent is ${ }_{1}^{7}$.

8. If the sine of an angle is $\frac{5}{13}$, find it cosine and its tangent.

9. If the cosine of an angle is $\frac{3}{5}$, find its sine and its tangent.

10. If the sine of an angle is $\frac{11}{6} \frac{1}{1}$, find its cosine and its tangent.

11. Divide the relation $\overline{B C}^{2}+\overline{A C}^{2}=\overline{A B}^{2}$ through by $\overline{A C}^{2}$, and what formula do you obtain ?

12. Prove that $(\sin A+\cos A)^{2}=1+2 \sin A \cos A$.

13. Prove that $\cos ^{2} A-\sin ^{2} A=2 \cos ^{2} A-1$. 
3. Numerical Values of the Ratios of Given Angles First, let the right triangle $A B C$ be such that the side $A C$ equals the side $B C$.

Then $\angle A=\angle B$, and each is half a right angle, or $45^{\circ}$.

If

then the measure of the side $A C=1$,

and the measure of the side $B C=1$, the measure of the side $A B=\sqrt{2}$. Why?

Hence $\sin 45^{\circ}=\frac{1}{\sqrt{2}}=.7071$,

$$
\begin{aligned}
& \cos 45^{\circ}=\frac{1}{\sqrt{2}}=.7071, \\
& \tan 45^{\circ}=\frac{1}{1}=1.0000 .
\end{aligned}
$$

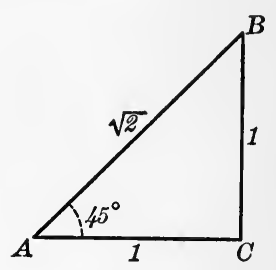

The decimal values given are computed correctly to four places.

Next, suppose that $\triangle A B D$ is equilateral. Then $\angle A=\angle B=\angle D$, and each is one-third of two right angles, or $60^{\circ}$.

From $B$ draw a perpendicular $B C$ to $A D$. This bisects both $\angle B$ and the side $A D$.

The measure of $\angle A B C$ is thus $30^{\circ}$.

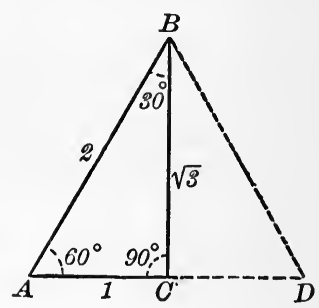

If the measure of each side of the equilateral triangle is two, then in $\triangle A B C$,

the measure of the side $A B=2$, of $A C=1$, and of $B C=\sqrt{3}$.

Hence

$$
\begin{aligned}
& \sin 60^{\circ}=\frac{B C}{A B}=\frac{\sqrt{3}}{2}=.8660 ; \\
& \cos 60^{\circ}=\frac{A C}{A B}=\frac{1}{2}=.5000 ; \\
& \tan 60^{\circ}=\frac{B C}{A C}=\frac{\sqrt{3}}{1}=1.7321 .
\end{aligned}
$$


Also,

$$
\begin{aligned}
& \sin 30^{\circ}=\frac{A C}{A B}=\frac{1}{2}=.5000 ; \\
& \cos 30^{\circ}=\frac{B C}{A B}=\frac{\sqrt{3}}{2}=.8660 ; \\
& \tan 30^{\circ}=\frac{A C}{B C}=\frac{1}{\sqrt{3}}=.5774 .
\end{aligned}
$$

By simple geometrical means, we have thus computed the sine, cosine, and tangent of angles whose measures are $30^{\circ}$, $45^{\circ}$, and $60^{\circ}$. It will be very easy to memorize the fractional values of these ratios if the diagrams from which they have been derived are firmly fixed in the mind.

For example, it should be noticed that in the triangle whose angles are $45^{\circ}, 45^{\circ}$, and $90^{\circ}$, the opposite sides are proportional to 1,1 , and $\sqrt{2}$; and in the triangle whose angles are $30^{\circ}, 60^{\circ}$, and $90^{\circ}$, the opposite sides are proportional to $1, \sqrt{3}$, and 2 .

Suppose that in the right triangle $C A B$, the hypotenuse $A B$ remains of fixed length, while $\angle A$ is gradually decreased by the rotation of $A B$ about the point $A$. The perpendicular $B C$ will become less and less, while the base $A C$ will increase in length. Finally when $\angle A=O^{\circ}, B C=O$, and $A C=A B$.

Hence $\sin O^{\circ}=\frac{B C}{A B}=O, \cos O^{\circ}=\frac{A C}{A B}=1, \tan O^{\circ}=\frac{B C}{A C}=O$.

Similarly by rotating $A B$ the other way until $\angle A=90^{\circ}$, it may be shown that sine $90^{\circ}=1, \cos 90^{\circ}=0, \tan 90^{\circ}=\infty$.

The trigonometric ratios of angles, or as they are frequently called, the trigonometric functions, have been carefully computed, by one means or another, for very minute subdivisions of the angles, and have been tabulated so as to be ready for use.

In the following table we give the sines, cosines, and tangents of angles from $0^{\circ}$ to $90^{\circ}$, at intervals of one degree, calculated correctly to four decimal places. 
TABLE OF TRIGONOMETRIC RATIOS

\begin{tabular}{|c|c|c|c|c|c|c|c|}
\hline Degrees & Sine & $\operatorname{Cosin} \theta$ & Tangent & Degrees & $\operatorname{Sin} \theta$ & Cosine & Tangent \\
\hline 0 & .0000 & 1.0000 & .0000 & 46 & .7193 & .6947 & 1.0355 \\
\hline 1 & .0175 & .9998 & .0175 & 47 & .7314 & .6820 & 1.0724 \\
\hline 2 & .0349 & .9994 & $.0: 349$ & 48 & .7431 & .6691 & 1.1106 \\
\hline 3 & .0523 & .9986 & .0524 & 49 & .7547 & .6561 & 1.1504 \\
\hline 4 & .0698 & .9976 & .0699 & 50 & .7660 & .6428 & 1.1918 \\
\hline 5 & .0872 & .9962 & .0875 & 51 & .7771 & .6293 & 1.2349 \\
\hline 6 & .1045 & .9945 & .1051 & 52 & .7880 & .6157 & 1.2799 \\
\hline 7 & $\cdot 1219$ & .9925 & .1228 & 53 & .7986 & .6018 & 1.3270 \\
\hline 8 & .1392 & .9903 & .1405 & 54 & .8090 & .5878 & 1.3764 \\
\hline 9 & .1564 & .9877 & .1584 & 55 & .8192 & .5736 & 1.4281 \\
\hline 10 & .1736 & .9848 & .1763 & 56 & .8290 & .5592 & 1.4826 \\
\hline 11 & .1908 & .9816 & .1944 & 57 & .8387 & .5446 & 1.5399 \\
\hline 12 & .2079 & .9781 & .2126 & 58 & .8480 & .5299 & 1.6003 \\
\hline 13 & .2250 & .9744 & .2309 & 59 & .8572 & .5150 & 1.6643 \\
\hline 14 & .2419 & .9703 & .2493 & 60 & .8660 & .5000 & 1.7321 \\
\hline 15 & .2588 & .9659 & .2679 & 61 & .8746 & .4848 & 1.8040 \\
\hline 16 & .2756 & .9613 & .2867 & 62 & .8829 & .4695 & 1.8807 \\
\hline 17 & .2924 & .9563 & .3057 & 63 & .8910 & .4540 & 1.9626 \\
\hline 18 & .3090 & .9511 & .3249 & 64 & .8988 & .4384 & 2.0503 \\
\hline 19 & .3256 & .9455 & .3443 & 65 & .9063 & .4226 & 2.1445 \\
\hline 20 & .3420 & .9397 & .3640 & 66 & .9135 & .4067 & 2.2460 \\
\hline 21 & .3584 & .9336 & .3839 & 67 & .9205 & .3907 & 2.3559 \\
\hline 22 & .3746 & .9272 & .4040 & 68 & .9272 & .3746 & 2.4751 \\
\hline 23 & .3907 & .9205 & .4245 & 69 & .9336 & .3584 & 2.6051 \\
\hline 24 & .4067 & .9135 & .4452 & 70 & .9397 & .3420 & 2.7475 \\
\hline 25 & .4226 & .9063 & .4663 & 71 & .9455 & .3256 & 2.9042 \\
\hline 26 & .4384 & .8988 & .4877 & 72 & .9511 & .3090 & 3.0777 \\
\hline 27 & .4540 & .8910 & .5095 & 73 & .9563 & .2924 & 3.2709 \\
\hline 28 & .4695 & .8829 & .5317 & 74 & .9613 & .2756 & 3.4874 \\
\hline 29 & .4848 & .8746 & .5543 & 75 & .9659 & .2588 & 3.7321 \\
\hline 30 & .5000 & .8660 & .5774 & 76 & .9703 & .2419 & 4.0108 \\
\hline 31 & .5150 & .8572 & .6009 & 77 & .9744 & .2250 & 4.3315 \\
\hline 32 & .5299 & .8480 & .6249 & 78 & .9781 & .2079 & 4.7046 \\
\hline 33 & .5446 & .8387 & .6494 & 79 & .9816 & .1908 & 5.1446 \\
\hline 34 & .5592 & .8290 & .6745 & 80 & .9848 & .1736 & 5.6713 \\
\hline 35 & .5736 & .8192 & .7002 & 81 & .9877 & .1564 & 6.3138 \\
\hline 36 & .5878 & .8090 & .7265 & 82 & .9903 & .1392 & 7.1154 \\
\hline 37 & .6018 & .7986 & .7536 & 83 & .9925 & .1219 & 8.1443 \\
\hline 38 & .6157 & .7880 & .7813 & 84 & .9945 & .1045 & 9.5144 \\
\hline 39 & .6293 & .7771 & .8098 & 85 & .9962 & .0872 & 11.4301 \\
\hline 40 & .6428 & .7660 & .8391 & 86 & .9976 & .0698 & 14.3007 \\
\hline 41 & .6561 & .7547 & .8693 & 87 & .9986 & .0523 & 19.0811 \\
\hline 42 & .6691 & .7431 & .9004 & 88 & .9994 & .0349 & 28.6363 \\
\hline 43 & .6820 & .7314 & .9325 & 89 & .9998 & .0175 & 57.2900 \\
\hline 44 & .6947 & .7193 & .9657 & 90 & 1.0000 & .0000 & $\infty$ \\
\hline 45 & .7071 & .7071 & 1.0000 & & & & \\
\hline
\end{tabular}




\section{EXERCISES}

1. In a right triangle the hypotenuse is 25 feet, and one adjacent angle is $32^{\circ}$. Find the other parts of the triangle.

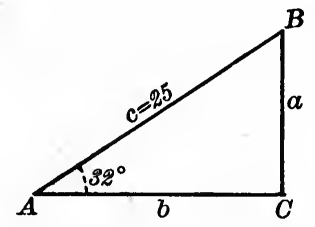

Solution. In the right triangle $A B C$, suppose $\angle A=32^{\circ}$ and $c=25$ feet.

Then, to find the side $a$,

$$
\frac{a}{c}=\sin A=\sin 32^{\circ},
$$

or

$$
a=c \cdot \sin 32^{\circ}=25 \times .5299=13.25 \text { feet nearly. }
$$

To find the side $b$,

$$
\frac{b}{c}=\cos A=\cos 32^{\circ} \text {, }
$$

or

$$
b=c \cdot \cos 32^{\circ}=25 \times .8480=21.20 \text { feet. }
$$

To find the angle $B$,

$$
B=90^{\circ}-A=90^{\circ}-32^{\circ}=58^{\circ} \text {. }
$$

Test the approximate accuracy of these results by showing

(1) $c^{2}=a^{2}+b^{2}$,

(2) $\tan B=\frac{b}{a}$.

2. In a right triangle one side is 36 feet and the adjacent angle is $54^{\circ}$. Find the other parts of the triangle.

3. In an isosceles triangle the base is 18 feet and the vertical angle $48^{\circ}$. Find its sides.

4. A regular pentagon inscribed in a circle has a side of 20 inches. Find the radius of the circle.

5. Find the length of the side of a regular octagon circumscribed about a circle of radius 15 inches. 
4. Ratios of Twice an Angle and Half an Angle

The following useful formulas can be easily deduced from well-known geometrical relations:

$$
\begin{aligned}
& \sin 2 A=2 \sin A \cos A \\
& \cos 2 A=\cos ^{2} A-\sin ^{2} A
\end{aligned}
$$

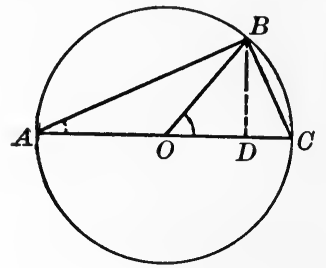

Let $O$ be the centre of a circle of which $A C$ is a diameter, and $A B C$ an inscribed triangle.

$\angle A B C$ is a right angle. Why? Draw $B D$ perpendicular to $A C$.

If

$$
\angle C A B=A, \angle C O B=2 A \text {. }
$$

Why?

First,

$$
\begin{aligned}
\sin 2 A & =\frac{B D}{O B}=\frac{2 B D}{A C}, \\
& =2 \frac{B D}{A B} \cdot \frac{A B}{A C}, \\
& =2 \sin A \cdot \cos A .
\end{aligned}
$$

Next,

$$
\cos 2 A=\frac{O D}{O B}=\frac{2 O D}{A C}=\frac{A D-D C}{A C},
$$

(since $A D=$ radius $+O D$, and $D C=$ radius $-O D$ ),

$$
\begin{aligned}
& =\frac{A D}{A C}-\frac{D C}{A C}, \\
& =\frac{A D}{A B} \cdot \frac{A B}{A C}-\frac{D C}{C B} \cdot \frac{C B}{A C} .
\end{aligned}
$$

Now

$$
\frac{A D}{A B}=\frac{A B}{A C} \text {, and } \frac{D C}{C B}=\frac{C B}{A C} \text {. }
$$


Therefore

$$
\begin{aligned}
\cos 2 A & =\left(\frac{A B}{A C}\right)^{2}-\left(\frac{C B}{A C}\right)^{2} \\
& =\cos ^{2} A-\sin ^{2} A
\end{aligned}
$$

If we remember that $\sin ^{2} A+\cos ^{2} A=1$, and in the last formula substitute for $\cos ^{2} A$ its value, viz., $1-\sin ^{2} A$, we obtain

$$
\cos 2 A=1-2 \sin ^{2} A \text {. }
$$

Or, if we substitute for $\sin ^{2} A$ its value, $1-\cos ^{2} A$, we obtain

$$
\cos 2 A=2 \cos ^{2} A-1 .
$$

If in these formulas $P$ is written in place of $2 A$, and consequently $\frac{P}{2}$ in place of $A$, they become

$$
\begin{aligned}
\sin P & =2 \sin \frac{P}{2} \cos \frac{P}{2} . \\
\cos P & =\cos ^{2} \frac{P}{2}-\sin ^{2} \frac{P}{2}, \\
& =1-2 \sin ^{2} \frac{P}{2}, \\
& =2 \cos ^{2} \frac{P}{2}-1 .
\end{aligned}
$$

The latter two formulas may be rewritten in the form,

$$
\begin{aligned}
& 2 \sin ^{2} \frac{P}{2}=1-\cos P \\
& 2 \cos ^{2} \frac{P}{2}=1+\cos P
\end{aligned}
$$

\section{EXERCISES}

1. From the last diagram produce the formula $\tan A=\frac{\sin 2 A}{1+\cos 2 A}$.

2. Deduce the values of the sine, cosine, and tangent of $15^{\circ}$ from the formulas given above, and compare your results with those given in the table.

3. Find in a similar way the values of the sine, cosine, and tangent of $22 \frac{1}{2}$ degrees. 


\section{Ratios of an Obtuse Angle}

Suppose $\angle B A C$ is obtuse. Then the perpendicular $P M$ drawn from a point in one boundary meets, not the other boundary, but the boundary produced backward through the vertex.

In that case, the base $A M$, i.e. the intercept between

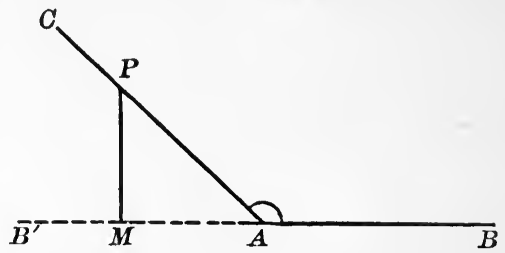
the vertex and the foot of the perpendicular, is considered to be negative. The perpendicular $P M$ and the hypotenuse $A P$ are considered positive, just as in the case of an acute angle.

The definitions of the functions of the obtuse angle $B A C$ are the same as for the acute angle $B A C$.

Hence, sine of the obtuse angle $B A C=\frac{M P}{A P}$, and is positive.

Cosine of the obtuse angle $B A C=\frac{A M}{A P}$, and is negative, since $A M$ is negative.

Tangent of the obtuse angle $B A C=\frac{M P}{A M}$, and is negative, since $A M$ is negative.

The functions of an acute angle are all positive since the perpendicular $P M$ falls upon the other boundary, and not upon that boundary produced backward.

The thing to be remembered in this connection is that if the perpendicular drawn from a point in one boundary of the angle meets the other boundary, the base is considered positive; but if it meets the other boundary produced backward through the vertex, the base is considered negative.

The sine of the obtuse angle $B A C$ is $\frac{M P}{A P}$; but this ratio is also the sine of the supplementary angle $B^{\prime} A C$, and for both angles the ratio is positive.

Hence, the sine of any angle equals the sine of its supplement. The cosine of the obtuse angle $B A C$ is $\frac{A M}{A P}$, which is also 
the cosine of the supplementary angle $B^{\prime} A C$. But for the latter angle the base $A M$ must be considered positive, since the perpendicular $P M$ meets the boundary $A B^{\prime}$ of that angle, while for the former angle $A M$ is negative.

That is, the cosine of an obtuse angle is equal in magnitude to the cosine of its supplement, but is negative. In other words, the cosine of any angle equals minus the cosine of its supplemient.

Similarly, the tangent of an obtuse angle is equal in magnitude to the tangent of its supplement, but is negative. In other words, the tangent of any angle equals minus the tangent of its supplement.

\section{EXERCISES}

1. Write the values of $\sin 120^{\circ}, \cos 135^{\circ}, \tan 150^{\circ}$.

2. What are the values of the sine, cosine, and tangent of an angle of $105^{\circ}$ ?

3. What is the difference in value between $\sin 45^{\circ}$ and $\sin 135^{\circ}$; between $\cos 45^{\circ}$ and $\cos 135^{\circ}$ ?

4. Find the value of $\sin 2 A$ when $\cos A=\frac{3}{5}$.

5. Find the value of $\cos 2 A$ when $\sin A=\frac{5}{13}$.

6. If $\tan A=\frac{5}{12}$, find the value of $\sin A, \cos A, \sin 2 A, \cos 2 A$.

7. If a chord $8 \mathrm{ft}$. in length is placed in a circle of $5 \mathrm{ft}$. radius, find approximately the size of the angle it subtends at the centre, and the length of its arc.

8. The sides of a right triangle are 5,12 , and 13 feet, respectively. Find the angles of the triangle. The mid-point of the side 12 is joined to the opposite vertex; into what two parts does the line so drawn divide the opposite angle?

9. The upper part of a tree broken off with the wind makes an angle of $30^{\circ}$ with the ground, and the distance from the root to the point where the top of the tree touches the ground is $50 \mathrm{ft}$. What was the height of the tree?

10. The angular elevation of the top of a chimney when viewed from one position is $32^{\circ}$, and on walking $96 \mathrm{ft}$. in a straight line toward the foot of the chimney the angular elevation of the top becomes $48^{\circ}$. Find the height of the chimney, 
6. Relations between the Sides and Axgles of a Triangle

1. In any triangle $\frac{\sin A}{a}=\frac{\sin B}{b}=\frac{\sin C}{c}$.

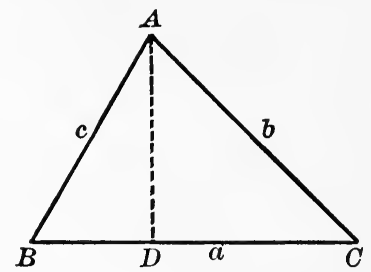

From any vertex $A$ of $\triangle A B C$ draw the perpendicular $A D$ to the opposite side.

Then $\quad \frac{A D}{A B}=\sin B$; hence $A D=A B \sin B=c \sin B$.

Also $\frac{A D}{A C}=\sin C$; hence $A D=A C \sin C=b \sin C$.

Therefore

$$
c \sin B=b \sin C \text {. }
$$

Dividing through by $b c$ gives

$$
\frac{\sin B}{b}=\frac{\sin C}{c} \text {. }
$$

If the perpendicular were drawn from the vertex $B$ to the opposite side, we should find in just the same way that

$$
\frac{\sin A}{a}=\frac{\sin C}{c} \text {. }
$$

Therefore

$$
\frac{\sin A}{a}=\frac{\sin B}{b}=\frac{\sin C}{c} \text {. }
$$

\section{EXERCISES}

1. What relation would be produced by drawing the perpendicular from the vertex $C$ ?

2. In a triangle $a=18 \mathrm{ft}$., $B=27^{\circ}, C=65^{\circ}$. Find the other parts of the triangle.

3. If $A=36^{\circ}, a=15 \mathrm{ft}$. and $b=24$, show that $B$ might have either of two values, and find those values approximately. 
2. In any triangle $\boldsymbol{c}^{2}=\boldsymbol{a}^{2}+\boldsymbol{b}^{2}-\boldsymbol{2} \boldsymbol{a b} \cos \boldsymbol{C}$.
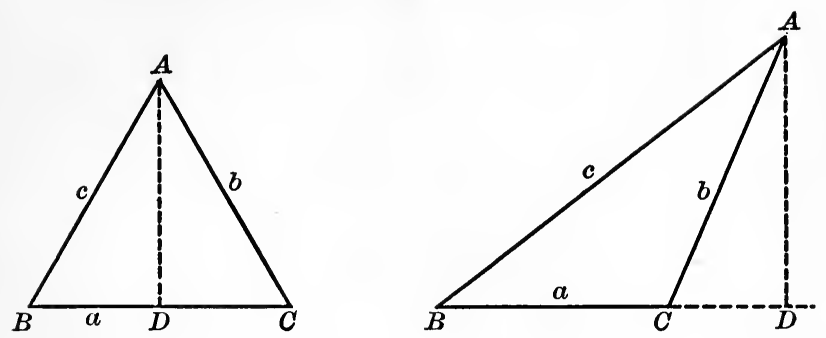

First, when the angle $C$ is acute,

$$
\overline{A B}^{2}=\overline{B C}^{2}+\overline{A C}^{2}-2 B C \cdot D C .
$$

But

$$
D C=A C \cos C \text {. }
$$

Therefore $\quad \overline{A B}^{2}=\overline{B C}^{2}+\overline{A C}^{2}-2 B C \cdot A C \cos C$,

$$
c^{2}=a^{2}+b^{2}-2 a b \cos C .
$$

Next, when the angle $C$ is obtuse,

$$
\overline{A B}^{2}=\overline{B C}^{2}+\overline{A C}^{2}+2 B C \cdot C D .
$$

But

$$
C D=A C \cos A C D=-A C \cos A C B .
$$

Therefore $\quad \overline{A B}^{2}=\overline{B C}^{2}+\overline{A C}^{2}-2 B C \cdot A C \cos C$,

or

$$
c^{2}=a^{2}+b^{2}-2 a b \cos C .
$$

Similarly,

$$
\begin{aligned}
& b^{2}=c^{2}+a^{2}-2 c a \cos B, \\
& a^{2}=b^{2}+c^{2}-2 b c \cos A .
\end{aligned}
$$

These formulas may be written, $\cos A=\frac{b^{2}+c^{2}-a^{2}}{2 b c}$, $\cos B=\frac{c^{2}+a^{2}-b^{2}}{2 c a}, \cos C=\frac{a^{2}+b^{2}-c^{2}}{2 a b}$. 
3. In any triangle $\sin \frac{A}{2}=\sqrt{\frac{(s-b)(s-\boldsymbol{c})}{b c}}$.

$$
\begin{aligned}
2 \sin ^{2} \frac{A}{2} & =1-\cos A \\
& =1-\frac{b^{2}+c^{2}-a^{2}}{2 b c}=\frac{2 b c-b^{2}-c^{2}+a^{2}}{2 b c} \\
& =\frac{a^{2}-(b-c)^{2}}{2 b c}=\frac{(a-b+c)(a+b-c)}{2 b c} .
\end{aligned}
$$

If, as in Art. 324, we let $a+b+c=2 s$,

then

$$
\begin{aligned}
& a-b+c=2 s-2 b=2(s-b), \\
& a+b-c=2 s-2 c=2(s-c) .
\end{aligned}
$$

Hence

$$
2 \sin ^{2} \frac{A}{2}=\frac{2(s-b) 2(s-c)}{2 b c},
$$

and

$$
\sin \frac{A}{2}=\sqrt{\frac{(s-b)(s-c)}{b c}} .
$$

4. In any triangle $\cos \frac{\boldsymbol{A}}{\mathbf{2}}=\sqrt{\frac{\boldsymbol{s}(\boldsymbol{s}-\boldsymbol{a})}{\boldsymbol{b} \boldsymbol{c}}}$.

$$
\begin{aligned}
2 \cos ^{2} \frac{A}{2} & =1+\cos A \quad \text { (Page 425.) } \\
& =1+\frac{b^{2}+c^{2}-a^{2}}{2 b c}=\frac{2 b c+b^{2}+c^{2}-a^{2}}{2 b c} \\
& =\frac{(b+c)^{2}-a^{2}}{2 b c}=\frac{(b+c+a)(b+c-a)}{2 b c} \\
& =\frac{2 s \cdot 2(s-a)}{2 b c} .
\end{aligned}
$$

Hence

$$
\cos ^{2} \frac{A}{2}=\frac{s(s-a)}{b c}
$$

and

$$
\cos \frac{A}{2}=\sqrt{\frac{s(s-a)}{b c}} .
$$


5. In any triangle $\boldsymbol{a}=\boldsymbol{b} \cos \boldsymbol{C}+\boldsymbol{c} \cos B$.
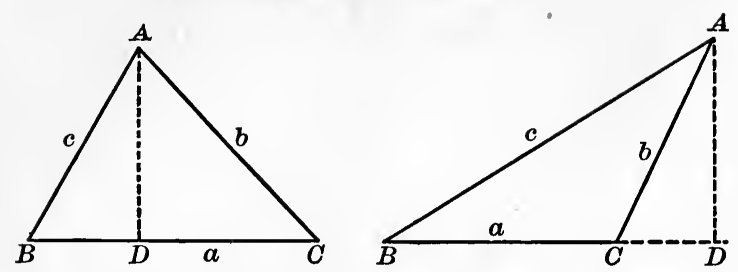

From the vertex $A$ draw the perpendicular $A D$ to the opposite side.

In case $B$ and $C$ are both acute angles,

$$
\begin{aligned}
a & =B D+D C \\
& =A B \cos B+A C \cos C \\
& =c \cos B+b \cos C .
\end{aligned}
$$

In case one angle, say $C$, is obtuse,

$$
\begin{aligned}
a & =B D-C D \\
& =A B \cos B-A C \cos A C D .
\end{aligned}
$$

But $\cos A C D=-\cos A C B$, since these are supplementary angles. The angle $A C B$ is $\angle C$ of the given triangle.

Therefore

$$
\begin{aligned}
a & =A B \cos B+A C \cos C . \\
& =c \cos B+b \cos C .
\end{aligned}
$$

\section{EXERCISES}

1. In a triangle whose sides are $7 \mathrm{ft}$., $9 \mathrm{ft}$., and $12 \mathrm{ft}$., find the angles (1) from their cosines, (2) from the sine of half of each angle.

2. Two adjacent sides of a triangle are 11 and $15 \mathrm{ft}$., respectively, and the included angle is $60^{\circ}$. Find the other parts.

3. The sides of a triangle are $10 \mathrm{ft}$., $13 \mathrm{ft}$., and $15 \mathrm{ft}$., respectively ; find the greatest and the least angle.

4. Two angles of a triangle are $36^{\circ}$ and $63^{\circ}$, respectively, and the greatest side is $20 \mathrm{ft}$; f find the remaining parts of the triangle.

5. Two sides of a triangle are $15 \mathrm{ft}$. and $18 \mathrm{ft}$., respectively, and the included angle is $105^{\circ}$. Find the third side. 


\section{Areas of Triangles}

1. The area of any triangle is equal to $\frac{1}{2} b c \sin \boldsymbol{A}$.
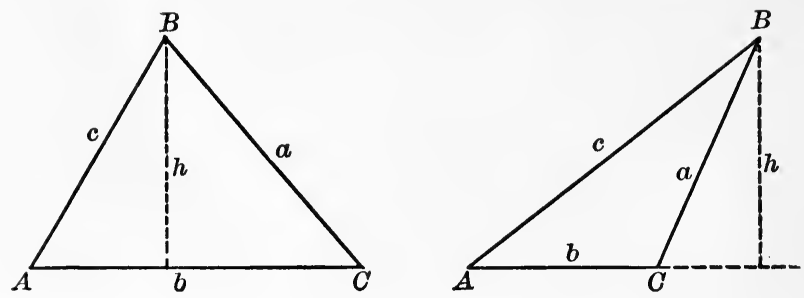

The area of a triangle $=\frac{1}{2} b h$, where $b$ is the side $A C$, and $h$ the altitude.

(Art. 306.)

But

$$
\frac{h}{c}=\sin A, \text { or } h=c \sin A .
$$

Substituting this value of $h$, we have

$$
\text { area }=\frac{1}{2} b c \sin A \text {. }
$$

Similarly it may be shown that

$$
\begin{aligned}
\text { area } & =\frac{1}{2} a b \sin C, \\
& =\frac{1}{2} a c \sin B .
\end{aligned}
$$

2. The area of any triangle equals $\sqrt{\boldsymbol{s}(\boldsymbol{s}-\boldsymbol{a})(\boldsymbol{s}-\boldsymbol{b})(\boldsymbol{s}-\boldsymbol{c})}$.

$$
\begin{aligned}
\text { area } & =\frac{1}{2} b c \sin A, \\
& =\frac{1}{2} b c \cdot 2 \sin \frac{A}{2} \cos \frac{A}{2} .
\end{aligned}
$$

But

$$
\sin \frac{A}{2}=\sqrt{\frac{(s-b)(s-c)}{b c}}, \text { and } \cos \frac{A}{2}=\sqrt{\frac{s(s-a)}{b c}} .
$$

Therefore $\quad$ area $=b c \sqrt{\frac{(s-b)(s-c)}{b c}} \cdot \sqrt{\frac{s(s-a)}{b c}}$,

$$
=\sqrt{s(s-a)(s-b)(s-c)} \text {. }
$$




\section{Inscribed and Circumscribed Circles}

1. The radius of the circle inscribed in a triangle equals $\frac{\boldsymbol{A}}{\boldsymbol{s}}$, where $A$ denotes the area of the triangle, and $s$, half the sum of the sides.

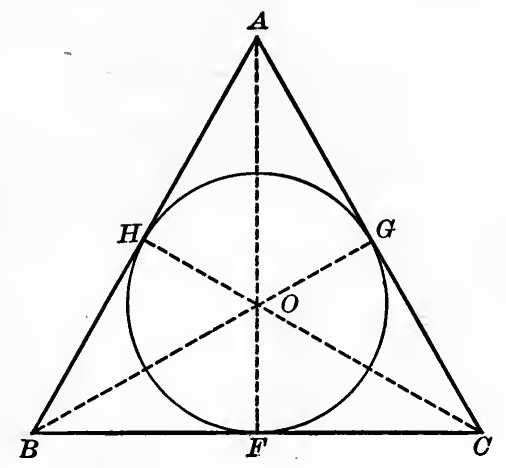

Let $O$ be the centre of the inscribed circle, and $O F, O G, O H$, radii drawn to the points of contact, and therefore perpendicular to the sides of the given triangle. Join $O A, O B, O C$.

$\triangle O B C+\triangle O C A+\triangle O A B=\triangle A B C$ whose area is $A$.

Area of $\triangle O B C=\frac{1}{2} O F \cdot B C=\frac{1}{2} r \cdot a$.

Area of $\triangle O C A=\frac{1}{2} O G \cdot C A=\frac{1}{2} r \cdot b$.

Area of $\triangle O A B=\frac{1}{2} O H \cdot A B=\frac{1}{2} r \cdot c$.

Therefore $A=\frac{1}{2} r(a+b+c)$,

$$
=r \cdot s
$$

Therefore

$$
r=\frac{A}{s} \text {. }
$$

In a similar way it may be shown that for a circle tangent to the side $a$ and to the sides $b$ and $c$ produced,

$$
r=\frac{A}{s-a} \text {. }
$$


2. The radius of the circle circumscribed about a triangle equals $\frac{\boldsymbol{a} \boldsymbol{b} \boldsymbol{c}}{\mathbf{4 A}}$.

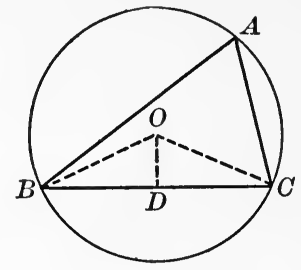

Let $O$ be the centre of the circumscribed circle, and $O D$ be drawn perpendicular to the side $B C$.

Then

$$
\begin{aligned}
\angle B O D & =\frac{1}{2} \angle B O C=\angle A . \\
\frac{B D}{B O} & =\sin B O D=\sin A, \\
\frac{1}{2} a & =R \sin A .
\end{aligned}
$$

or

Therefore

$$
R=\frac{a}{2 \sin A}=\frac{a b c}{2 b c \sin A}=\frac{a b c}{4 A} .
$$

\section{EXERCISES}

1. If the sides of a triangle are 56,65 , and $33 \mathrm{ft}$., find the greatest angle and the area of the triangle.

2. The sides of a parallelogram are 15 and $17 \mathrm{ft}$., and one of its smaller angles is $42^{\circ}$. Find its area and the lengths of its diagonals.

3. The sides of a triangular field are 119, 111, and $92 \mathrm{yds}$. Show that its area is $10 \mathrm{sq}$. yds. less than an acre.

4. Two angles of a triangle are $42^{\circ}$ and $68^{\circ}$, respectively, and the least side is $20 \mathrm{ft}$. Find its area.

5. The sides of a triangle are $11 \mathrm{ft}$., $13 \mathrm{ft}$., and $18 \mathrm{ft}$., respectively. Find the radius of the inscribed circle.

6. Two sides of a triangle are $21 \mathrm{ft}$. and $24 \mathrm{ft}$., respectively, and the included angle is $110^{\circ}$. Find its area. 


\section{MENSURATION FORMULAS}

In the following table, unless otherwise specified, $A$ denotes the area; $a, b, c$, the sides of a triangle; $b$, the base; $c$, the hypotenuse of a right triangle ; $r$, the radius; $d$, the diameter; $l$, the lateral edge; $s$, the slant height; $C$, the circumference; $P$, the perimeter; $L$, the lateral area; $V$, the volume.

1. In any parallelogram

$$
A=b h .
$$

Art. 305

2. In a right triangle $c^{2}=a^{2}+b^{2}$. Art. 317

3. In any triangle $A=\frac{1}{2} b h$.

Art. 306 $A=\sqrt{s(s-a)(s-b)(s-c)} . \quad$ Art. 324, Pg. 432

$A=\frac{1}{2} b c \sin A$. Pg. 432

$a^{2}=b^{2}+c^{2}-2 b c \cos A$.

Pg. 429

$$
\frac{\sin A}{a}=\frac{\sin B}{b}=\frac{\sin C}{c} \text {. }
$$

Pg. 428

$$
\sin \frac{A}{2}=\sqrt{\frac{(s-b)(s-c)}{b c}} .
$$

4. In a circle

$$
\begin{aligned}
& C=\pi d=2 \pi r . \\
& A=\frac{1}{2} C r=\pi r^{2}=\frac{1}{4} \pi d^{2} .
\end{aligned}
$$

Art. 365

5. In a regular polygon

$$
A=\frac{1}{2} P a, \text { where } a \text { is the apothem. }
$$

Art. 353

6. In a parallelepiped or any prism

$$
L=l P,
$$

where $P$ is the perimeter of a right section.

$$
V=h A \text {, }
$$

where $A$ is the area of the base. 
7. In a regular pyramid

$$
L=\frac{1}{2} P s,
$$

where $P$ is the perimeter of the base.

Art. 512

$$
V=\frac{1}{3} h A, \cdot
$$

where $A$ is the area of the base.

Art. 523

8. In a circular cylinder

$$
L=l P,
$$

where $P$ is the perimeter of a right section.

Art. 560

$$
V=\pi r^{2} h \text {. }
$$

Art. 562

9. In a right circular cone

$$
L=\pi r s,
$$

where $r$ is the radius of the base.

Art. 577

$$
V=\frac{1}{3} \pi r^{2} h \text {. }
$$

Art. 579

10. In a sphere

$$
\begin{aligned}
& A=4 \pi r^{2} . \\
& A=720 \text { spherical units. } \\
& V=\frac{4}{3} \pi r^{3}=\frac{1}{3} A r .
\end{aligned}
$$

Art. 661

Art. 652

Art. 667

11. In a spherical segment

$$
\begin{aligned}
& L=2 \pi r h . \\
& V=\frac{1}{2} h\left(\pi a^{2}+\pi b^{2}\right)+\frac{1}{6} \pi h^{3},
\end{aligned}
$$

where $a$ and $b$ are the radii of the two bases. 


\section{INDEX TO DEFINITIONS}

The numbers refer to pages

Acute angle, 11.

Adjacent, angles, 8. polygons, 194 .

Altitude, 197, 198, 311, 329.

Angle, 6.

acute, 11.

cosine of, 416 .

dihedral, 284.

in an arc, 111.

obtuse, 11.

polyhedral, 296.

reflex, 12 .

right, 9.

sine of, 416.

spherical, 380 .

straight, 12.

tangent of, 416.

trihedral, 296.

Angles, adjacent, 8.

alternate, 62 .

complementary, 67 .

supplemetary, 11.

of a triangle, 7 .

vertical, 8.

Apothem, 239.

Arc of a circle, 100.

length of, 245.

Arcs, similar, 249.

Area, 195, 245, 394.

Axiom, 16.

Axis, of a circle, 372.
Axis, radical, 170.

of symmetry, 88 .

Centroid of a triangle, 80 .

Chord, of a circle, 100.

of contact, 119.

Circle, 14.

arc of, 100.

centre of, 14.

chord of, 100.

diameter of, 14.

radius of, 14 .

secant of, 115 .

sector of, 101.

segment of, 101.

tangent of, 116.

Circles, coaxial system of, 170 . concentric, 18.

in contact, 130 .

Circular, cone, 361 .

cylinder, 354.

Circumference of a circle, 244 .

Circumscribed figure, 119.

Closed surface, 310 .

Coaxial circles, 170.

Commensurable magnitudes, 141.

Common tangents, direct and inverse, 133.

Concentric, circles, 18.

spheres, 370.

Concurrent lines, 79 . 
Concyclic points, 114.

Cone, 361.

frustum of, 365 .

right circular, 362.

Conical surface, 361.

Conjugate arcs, 101.

Contact, chord of, 119, circles in, 130.

Continued proportion, 152.

Continuity, principle of, 125.

Converse theorems, 32 .

Corollary, 35.

Corresponding angles, 62.

Cosine of an angle, 416.

Cube, 317.

Cylinder, 354.

right circular, 354 .

Cylindrical surface, 353.

Decagon, 233.

Diagonal, 71.

Diagonals, principal, 252.

Diameter, 14.

Dihedral angle, 284. plane angle of, 285.

Distance from a point, to a straight line, 53.

to a plane, 275 .

Dodecagon, 233.

Dodecahedron, 311.

Equilateral triangles, 22.

Extreme and mean ratio, 177.

Extremes, 149.

Figure, circumscribed, 119.

closed, 13.

inscribed, 111 .

plane, 5 .

rectilinear, 13.

solid, 263.
Figures, identically equal, 9. isoperimetric, 228. symmetrical, 88.

Frustum, of a cone, 365 . of a pyramid, 328 .

Great circle, 371.

Harmonic division, 186.

Hexagon, 233. principal diagonals of, 252.

Hexahedron, 311.

Homologous sides, 30.

Hypotenuse, 44.

Icosahedron, 311.

Incommensurable magnitudes, 141.

Indirect proof, 47.

Inscribed figure, 111.

Inverse points, 88.

Isoperimetric figures, 228.

Isosceles, triangle, 22. trihedral angle, 302 .

Length of an arc, 245.

Limit, 147.

Line, oblique to a plane, 266. parallel to a plane, 266 . perpendicular to a plane, 266 . straight, 3.

Line-segments, 3.

Lines, concurrent, 79. parallel, 61. perpendicular, 9 . skew or gauche, 271.

Locus, 50.

Lune, 395.

angle of, 395 .

Magnitude, 16.

Maximum value, 111, 226. 
Mean proportional, 152.

Means, 149.

Measure, 140.

Median of a triangle, 79.

Minimum value, 111, 226.

Multiple, 140.

Octagon, 233.

Octahedron, 311.

Orthocentre of a triangle, 83.

Parallel, lines, 61. planes, 266.

Parallelepiped, 317. rectangular, 317. right, 317.

Parallelogram, 74. altitude of, 197.

Pentagon, 233.

Pentahedron, 311.

Perimeter, 79.

Perpendicular, to a line, 9. to a plane, 266.

Plane surface, 4.

Points, concyclic, 114. inverse, 88.

Polar spherical triangles, 387.

Poles of a circle, 372.

Polygon, 71, 73. convex, 71, 73 . diagonal of, 71 . opposite sides of, 72 . perimeter of, 79 .

regular, 233. spherical, 382.

Polygons, adjacent, 194. mutually equiangular, 161 . similar, 161.

Polyhedral angle, 296.

Polyhedral angles, symmetrical, 297. Polyhedron, 310. circumscribed, 378.
Polyhedron, convex, 310. inscribed, 378. regular, 342.

Polyhedrons, similar, 339.

Postulate, 15.

Principle of continuity, 125.

Prism, 311. truncated, 315.

Problem, 17.

Projection, 212, 292.

Proportion, 149.

Pyramid, 328.

Quadrant, 372.

Quadrilateral, 73.

Radical axis, 170.

Radius, 14.

Ratio, 142, 144, 148.

extreme and mean, 177 .

Rectangle, 74.

Regular polygon, 233. apothem of, 239. centre of, 239. radius of, 239.

Regular pyramid, 328. slant height of, 328 .

Regular polyhedron, 342.

Rhombus, 74 .

Right angle, 9.

Right triangle, 44.

Right circular, cone, 362 . cylinder, 354 . prism 311.

Scalene triangles, 22.

Secant of a circle, 115.

Section of a surface, $\mathbf{3 1 0}$.

Sector, of a circle, 101. of a sphere, 406.

Segment, of a circle, 101, of a sphere, 401 . 
Semicircle, 101.

Similar, arcs, 249.

polygons, 161.

polyhedrons, 339 .

sectors, 250.

segments, 249.

Sine of an angle, 416.

Sphere, 370.

Spherical, angle, 380.

excess, 391 .

polygon, 382 .

pyramid, 406.

sector, 406.

segment, 401.

triangle, 382 .

unit of surface, 397 .

Square, 74.

Surface, closed, 310.

conical, 361.

cylindrical, 353.

plane, 4.

Symmetrical, figures, 88.

polyhedral angles, 297.

spherical triangles, 384 .

Symmetry, axis of, 88 .

Tangent, of an angle, 416.

to a circle, 116.

to a cone, 362 .
Tangent, to a cylinder, 355.

to a sphere, 375 .

Tangents, common, direct and inverse, 133.

Tetrahedron, 311.

Theorem, 17.

Third proportional, 152.

Transversal, 62.

Trapezium, 74.

Trapezoid, 74.

isoceles, 77.

Triangle, 5.

altitude of, 198.

centroid of, 80.

equilateral, 22.

isosceles, 22.

median of, 79 .

right, 44.

scalene, 22.

spherical, 382.

Trihedral angle, 296.

isosceles, 302.

Truncated, prism, 315.

pyramid, 328 .

Vertex, 5, 71, 310.

Volume of a polyhedron, 315,324 .

Zone, 401. 



\section{UNIVERSITY OF CALIFORNIA LIBRARY BERKELEY}

Return to desk from which borrowed.

This book is DUE on the last date stamped below.

NOV 2947 6May'55\%W

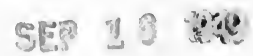

WAY $61955 L 4$

SEF 171953

$20 r^{\prime} b^{\prime} 60 \mathrm{Cr}$

SEP $10195 \%$ LU

$F=3$; 6 ind 


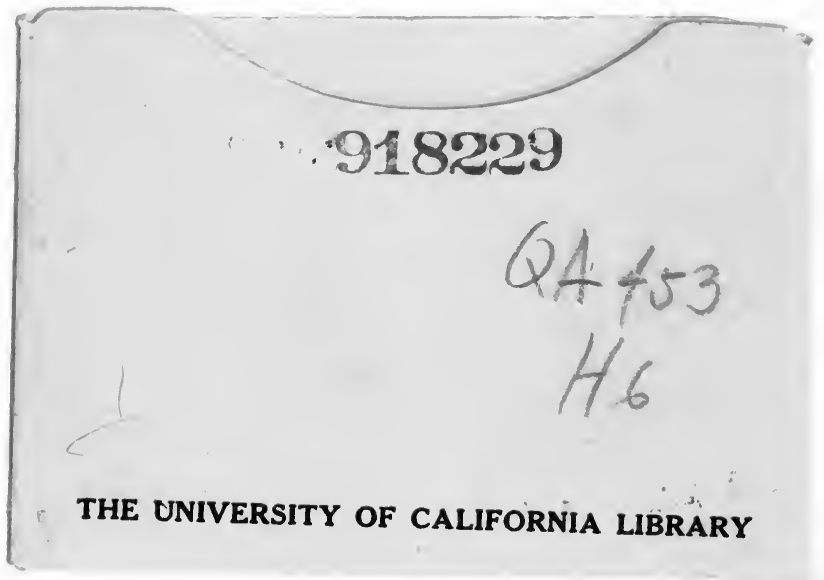


